\title{
Darstellung bor- und phosphorhaltiger Ringe durch Reaktionen von
}

\section{Halogenboranen und -phosphanen mit}

\section{Bis(tert.-butyl-methyl)ketazin}

\author{
Dissertation \\ zur Erlangung des Doktorgrades \\ der Mathematisch-Naturwissenschaftlichen Fachbereiche \\ der Georg-August-Universität Göttingen
}

vorgelegt von

Florian Armbruster

aus Heidelberg 
D 7

Referent: Prof. Dr. F. Meyer

Korreferent: Prof. Dr. U. Klingebiel

Tag der mündlichen Prüfung: 28.04.2004 
Wer alle seine Ziele erreicht hat, der hat sie zu niedrig gewählt!

Für Nina 


\section{Inhaltsverzeichnis}

Inhaltsverzeichnis

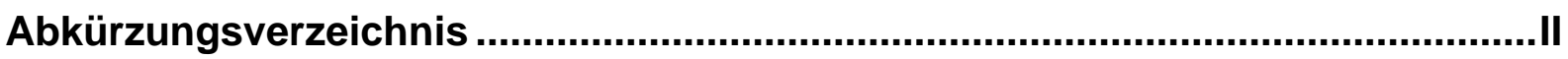

1 Einleitung

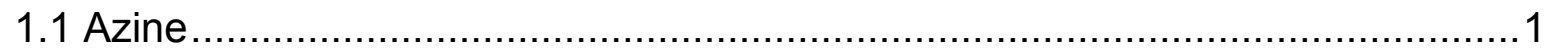

1.1.1 Allgemeine Eigenschaften und Verwendung der Azine .............................2

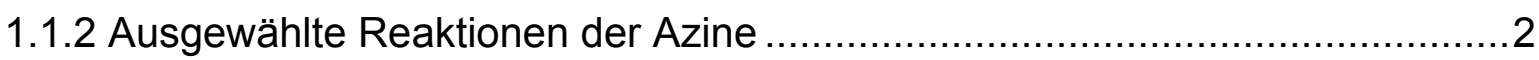

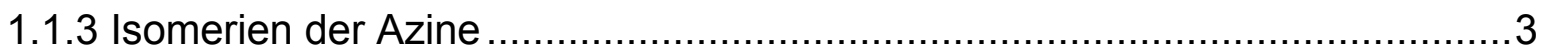

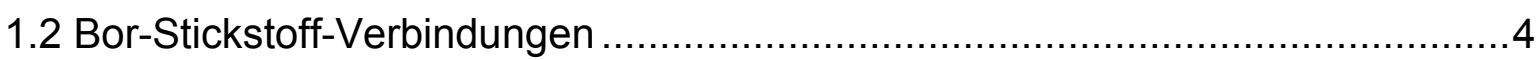

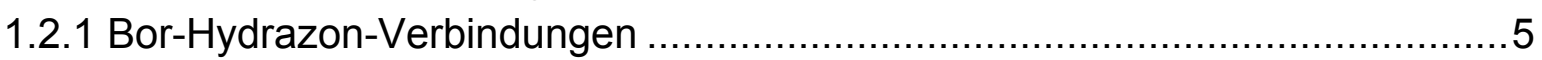

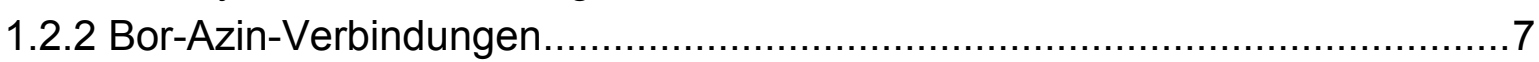

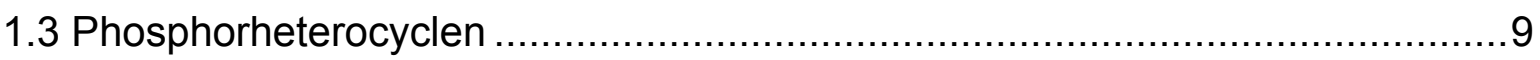

1.3.1 Auswahl bekannter Typen von Phosphor-Heterocyclen ..............................

1.3.2 Fünfgliedrige phosphor- und stickstoffhaltige Ringe ...............................10

1.3.3 1,2-Diaza-3-phosphacyclopent-5-ene und -penta-3,5-diene........................11

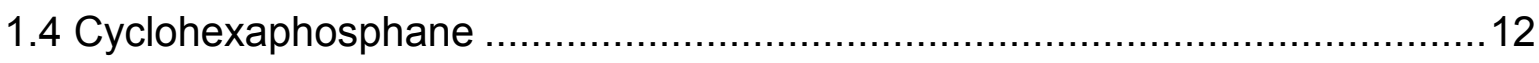

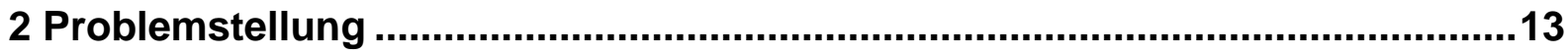

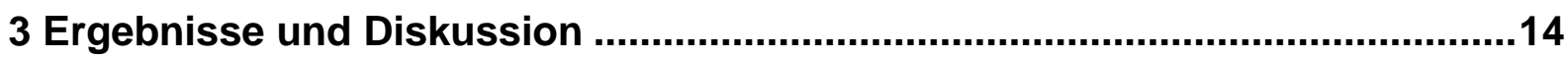

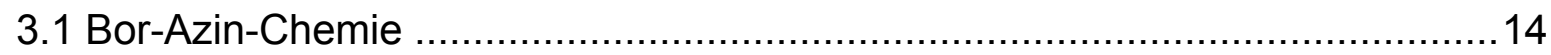

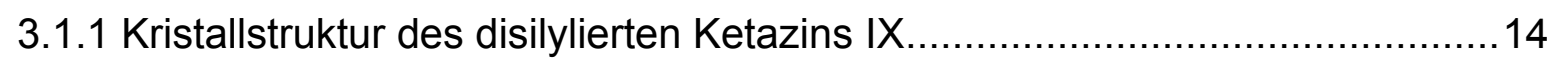

3.1.2 Synthese weiterer fünfgliedriger borhaltiger Ringe ...............................17

3.1.3 Kristallstruktur des 2,6-tert.-Butoxi-4,8-diisopropyl-1,5-diaza-2,6-dibora-

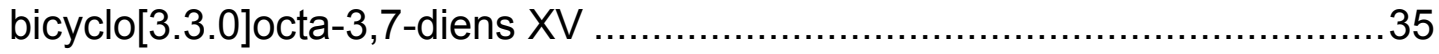

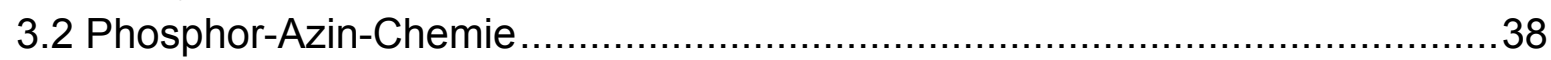

3.2.1 Synthese von fünfgliedrigen phosphorhaltigen Ringen ...............................38

3.2.2 Versuchte Darstellung eines acyclischen Phosphylketazins........................49

3.2.3 Synthese achtgliedriger phosphorhaltiger Ringe ....................................53

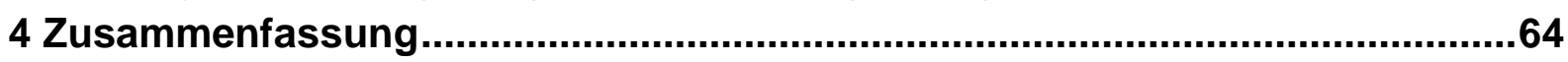

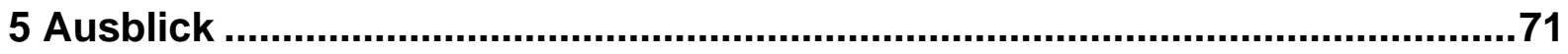

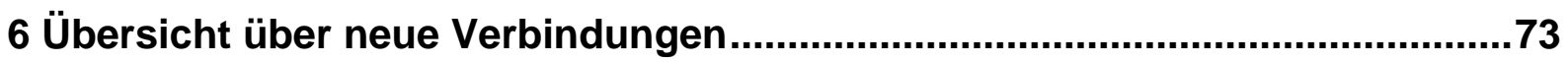

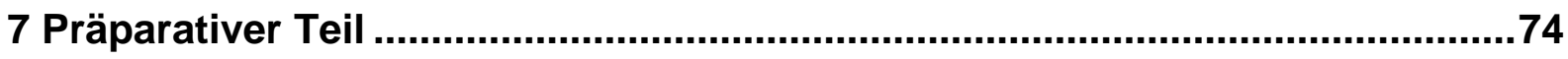

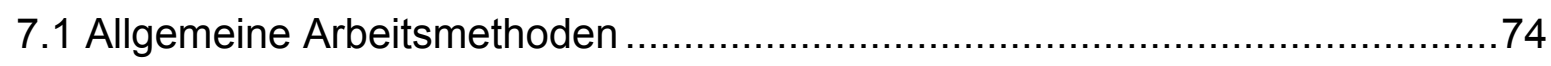

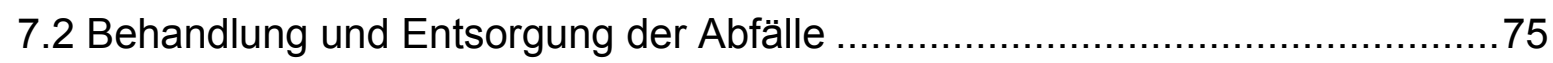

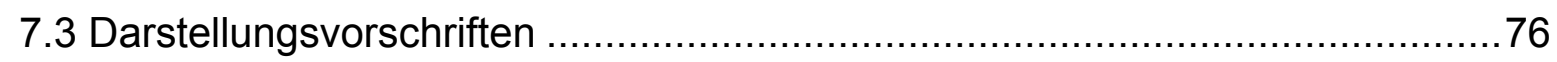

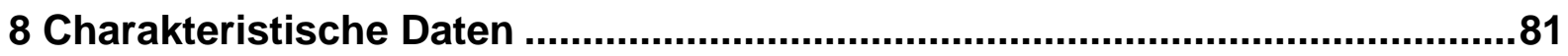

8.1 Namen, physikalische und chemische Eigenschaften ..................................

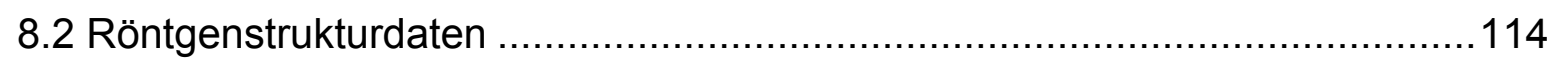

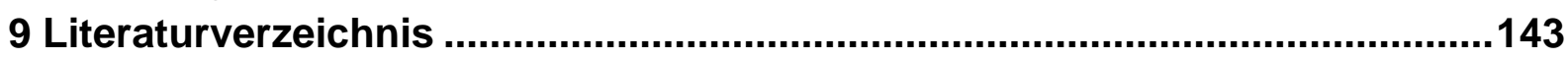

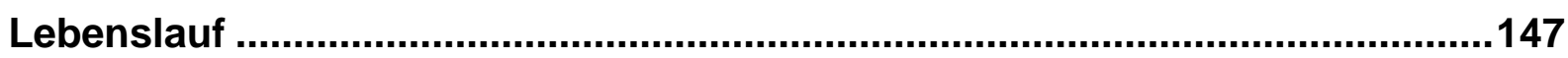

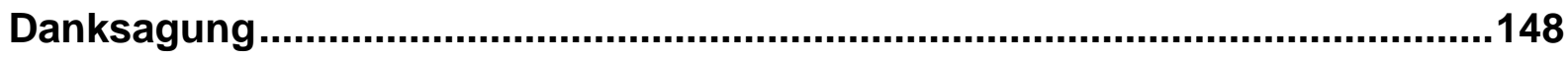




\section{Abkürzungsverzeichnis}

$\AA \quad$ Angstrøm, $1 \AA=10^{-4} \mathrm{~m}=100 \mathrm{pm}$

AA'XX' NMR-Spektrum höherer Ordnung

ABX NMR-Spektrum höherer Ordnung

Äq. Äquivalent, Äquivalente

ber. berechnet

br. breites NMR-Signal

$\mathrm{Bu} \quad$ Butyl $\left(-\mathrm{C}_{4} \mathrm{H}_{9}\right)$

$\mathrm{C}_{\mathrm{t}} \quad$ ternäres Kohlenstoffatom

$\mathrm{Cr}$ (acac) Chromacetylacetonat $\left(\mathrm{C}_{5} \mathrm{H}_{7} \mathrm{O}_{2} \mathrm{Cr}\right)$

$\delta \quad$ chemische Verschiebung

$\Delta \quad$ Wärmezufuhr

d day, Tag

d. Dublett

dvd. Dublett von Dublett

$\mathrm{d} \boldsymbol{v} \mathrm{d} \boldsymbol{v d}$. Dublett von Dublett von Dublett

d $\boldsymbol{v d} \boldsymbol{v d e c} \boldsymbol{v}$. Dublett von Dublett von Dezett von Dublett

dvsept. Dublett von Septett

EI Elektronenstoßionisation

$\mathrm{eV} \quad$ Elektronenvolt

gek. gekoppelt aufgenommene NMR-Spektren

h hour, Stunde

Hal Halogen

HV Hausvakuum ( 30 mbar $)$

iPr Isopropyl $\left(-\mathrm{CH}\left(\mathrm{CH}_{3}\right)_{2}\right)$

$\mathrm{J} \quad$ Kopplungskonstante

$[\mathrm{M}]^{+} \quad$ Molekülion

m. Multiplett

Me Methyl $\left(-\mathrm{CH}_{3}\right)$

$n$ - normal, unverzweigt

ND Normaldruck (1013 mbar)

NMR nuclear magnetic resonance, Kernspinresonanzspektroskopie

ÖV Ölpumpenvakuum ( 10 ${ }^{-2}$ mbar $)$

$\mathrm{Ph} \quad$ Phenyl $\left(-\mathrm{C}_{6} \mathrm{H}_{5}\right)$

ppm parts per million

pst. pseudo-Triplett 
$\mathrm{R} \quad$ beliebiger organischer Rest

RT Raumtemperatur $\left(\sim 20^{\circ} \mathrm{C}\right)$

S. Singulett

sbr. sehr breites NMR-Signal

Schmp. Schmelzpunkt

Sdp. Siedepunkt

sept. Septett

sMes Supermesityl, 2,4,6-Tris(tert.-butyl)phenyl $\left(-\mathrm{C}_{6} \mathrm{H}_{2}\left(\mathrm{C}\left(\mathrm{CH}_{3}\right)_{3}\right)_{3}\right)$

Subp. Sublimationspunkt

t. Triplett

$t-$, tert.- $\quad$ tertiär

THF Tetrahydrofuran $\left(\mathrm{C}_{4} \mathrm{H}_{8} \mathrm{O}\right)$

TMS Tetramethylsilan $\left(\mathrm{Si}\left(\mathrm{CH}_{3}\right)_{4}\right)$

$\mathrm{X}$ beliebiges Element

Die vorgestellten Verbindungen werden fortlaufend nummeriert. Bekannte Verbindungen werden römisch beziffert, bis dato nicht isolierte Verbindungen arabisch.

Arabisch fett gedruckte unterstrichene Verbindungen sind charakterisiert, arabisch bezifferte Verbindungen ohne Formatierung sind hypothetisch oder konnten nicht dargestellt bzw. isoliert werden. Bekannte Verbindungen, die um neue Daten ergänzt wurden, sind mit römischen fett gedruckten Ziffern nummeriert.

Die Verbindungen sind nach den Regeln der IUPAC in Bezug auf Alkane, Borane bzw. Phosphane benannt. Um die Transparenz zu der nah verwandten organischen Chemie zu erhöhen sind im Anhang die dort üblichen Benennungen mit angegeben, z.B. 1,2,3-Diazaborol (bzw. in engl. 1,2,3-diazaborole) statt 1,2-Diaza-3-boracyclopent-5-en. Im Text werden beide Namen parallel verwendet. 


\section{Einleitung}

\subsection{Azine}

Azine sind Derivate des Hydrazins. Sie weisen folgende allgemeine Struktur ${ }^{1}$ auf:<smiles>[R]C([R])=NN=C([R3])[R]</smiles>

Abbildung 1: Allgemeine Struktur eines Azins

Dabei können die Reste $\mathrm{R}_{1}$ bis $\mathrm{R}_{4}$ Wasserstoff, Acyl-, Aryl- oder Alkylreste sein. Üblicherweise stellt man Azine durch eine doppelte Kondensation aus Hydrazin mit Carbonylverbindungen dar. Bei der Verwendung von Ketonen entstehen Ketazine, beim Einsetzen von Aldehyden Aldazine. Diese Arbeit befasst sich mit Ketazinen als Ausgangssubstanz. Die symmetrischen Azine mit $R_{1}=R_{4}$ und $R_{2}=R_{3}$ sind in einem Schritt zugänglich:
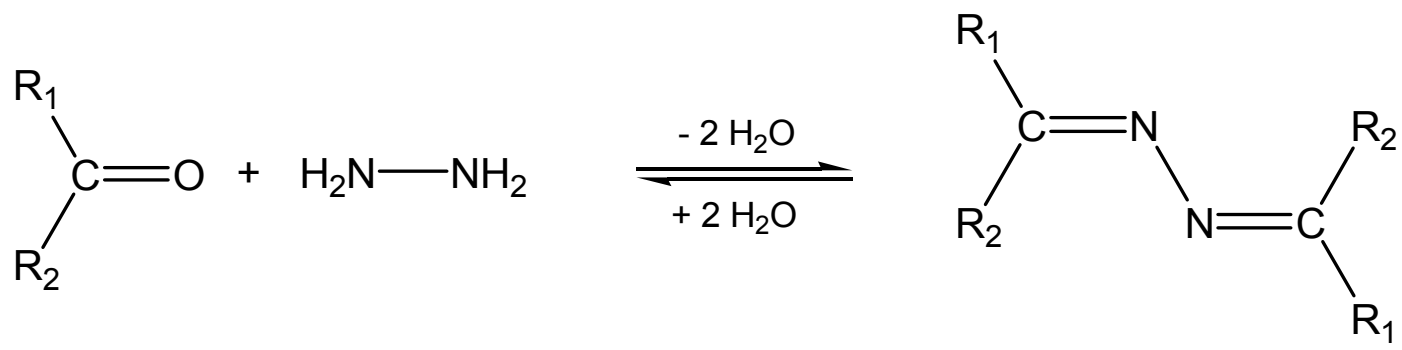

Schema 1: Gleichgewichtsreaktion von Hydrazin mit Ketonen

Bei unvollständiger (also einfacher) Kondensation entstehen die nahe verwandten Hydrazone ${ }^{2}$. Sie sind NH-funktionell. Das Gleichgewicht lässt sich durch Zugabe von Säuren zu einem Keton-Hydrazin-Gemisch in Richtung der Ketazine verschieben, durch Zugabe von Basen wie Calciumoxid in Richtung der Hydrazone. Im sauren 
Milieu ist also die Bildung von Ketazinen bevorzugt, in einer wasserentziehenden basischen Reaktionsumgebung die Bildung von Hydrazonen.

Weitere Synthesewege zur Darstellung von Azinen sind bekannt. Darunter ist eine Methode zur Darstellung symmetrischer Azine der oxidative Aufbau der C=N-Doppelbindung aus Carbonylverbindung mit Ammoniak in Anwesenheit eines Oxidationsmittels (z.B. $\mathrm{H}_{2} \mathrm{O}_{2}$ ) und eines Katalysators (z.B. Kieselgel) in polaren organischen Lösungsmitteln (z.B. Acetonitril, Isopropanol) ${ }^{1}$.

\subsubsection{Allgemeine Eigenschaften und Verwendung der Azine}

Die Chemie der Azine begründet sich auf T. CURTIUS, der diese Stoffklasse Anfang des letzten Jahrhunderts entdeckte und untersuchte ${ }^{3}$. Azine aliphatischer Ketone sind schwach gefärbte Flüssigkeiten, Azine von aromatischen Carbonylverbindungen und Heterocyclen sind zumeist Feststoffe. Die Löslichkeit ist in organischen Lösungsmitteln gut, in Wasser lösen sich die meisten Azine nicht. Alle besitzen im UV-Spektrum charakteristische Absorptionsbanden und viele sind fluoreszenzfähig, was mit der Fähigkeit der Azine Wasserstoffbrücken auszubilden und der Koplanarität der Atome in der Azin-Gruppe in Zusammenhang steht ${ }^{1}$.

Eine Reihe bereits synthetisierter Azine weist antibakterielle oder tuberkulostatische Wirkungen auf. Einzelne dienen der Herstellung anticancerogener Substanzen.

In der Industrie finden Azine vielfältige Verwendung. Die größte Bedeutung haben sie als Stabilisatoren für Benzin, Seifen, chlorierte Kohlenwasserstoffe und Industrieöle und bei der Verarbeitung von Kunststoffen als Festigungsmittel ${ }^{1}$.

In der Analytik werden Azine als Nachweisreagenzien für Metalle (z.B. Kupfer, Zink) und als Indikatoren bei potentiometrischen Titrationen verwendet.

\subsubsection{Ausgewählte Reaktionen der Azine}

Gegenüber Basen sind Azine unter moderaten Bedingungen (RT, ND) stabil, bei stärkerer Druck- oder Temperaturerhöhung zersetzen sie sich in die Ausgangssubstanzen ${ }^{4}$. Mit Mineralsäuren bilden Azine Azinium-Salze. Sie liefern stabile Metallkomplexe, die bis über $250{ }^{\circ} \mathrm{C}$ beständig sind. Azine aliphatischer Aldehyde und Ket- 
one können unter Einwirkung wasserfreier Säuren zu Pyrazolinen cyclisieren. Die wichtigsten Cycloadditionsreaktionen sind die 1,3-dipolaren Cycloadditionen ${ }^{1,3}$.

Ketazine mit einem aciden Wasserstoff am $\alpha$-C-Atom können unter Einwirkung starker Basen 1,6-Dianionen bilden ${ }^{5}$ :
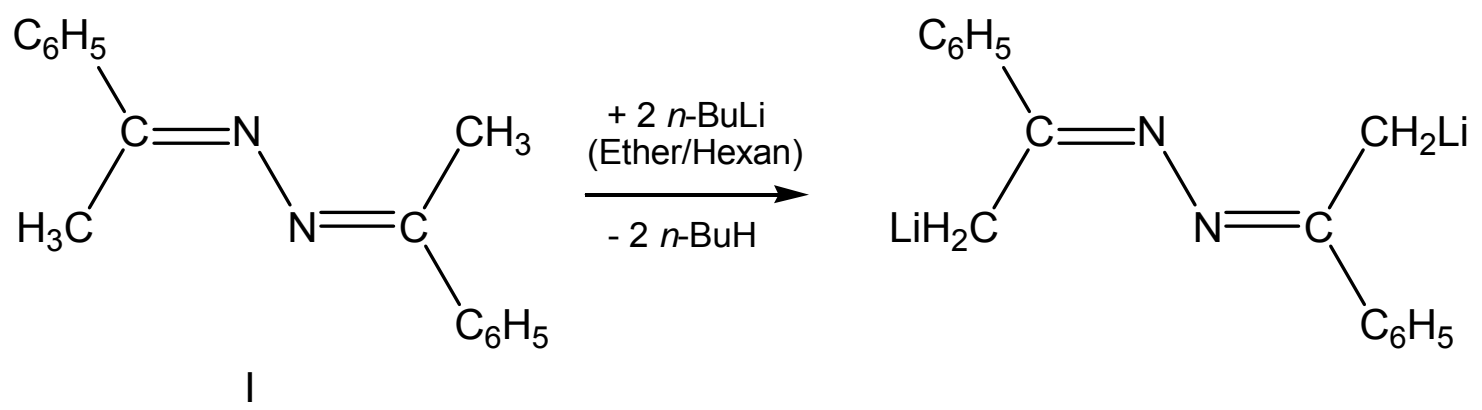

Schema 2: Metallierung von Azinen unter Verwendung starker Basen

Obwohl die Dianionen selbst noch nicht isoliert oder kristallisiert werden konnten, sind Folgeprodukte isoliert, die ihre Existenz bestätigen. Die Umsetzung der Dianionen mit Halogenboranen wurde im Arbeitskreis MELLER ${ }^{6,7,8}$ untersucht (siehe Kapitel 1.2.2).

\subsubsection{Isomerien der Azine}

Die Isomerie der Azine entspricht der $\mathrm{C}=\mathrm{N}$-Isomerie ${ }^{9}$. Eine Imin-Enamin-Tautomerie ist ebenso wie die photochemische (E/Z)-Isomerisierung eingehend untersucht. Auch für symmetrische Azine sind Isomere bekannt:

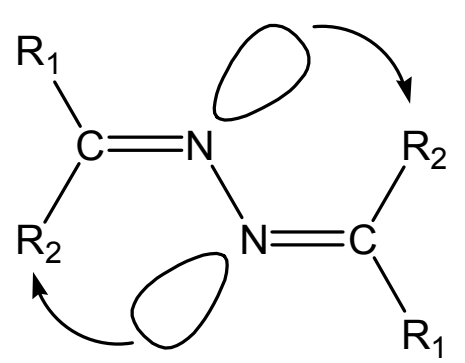

A

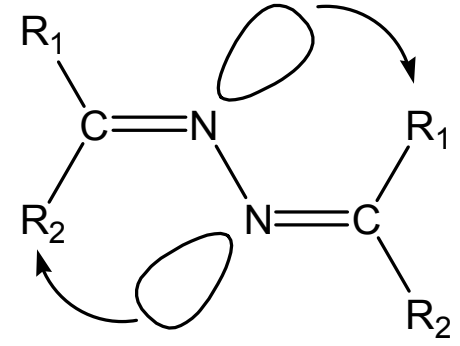

B

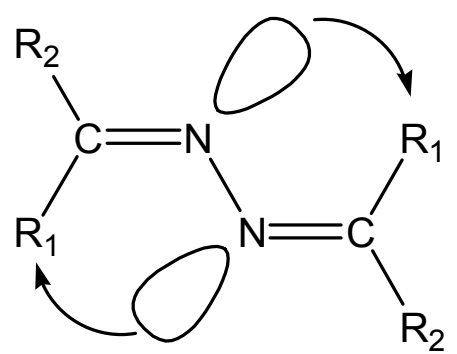

C

Abbildung 2: Isomerietypen eines symmetrischen Azins 
In Bezug auf die Nähe des freien Elektronenpaares am Stickstoffatom zu $R_{1}$ werden die Isomere als Anti-Anti- (A), Anti-Syn- (B) und Syn-Syn-Konformationen (C) bezeichnet ${ }^{10,11,12}$.

In dieser Arbeit wurde der Methyl-Rest als Substituent $\mathrm{R}_{1}$ gewählt um eine H-acide Funktion zur Verfügung zu haben (vgl. Schema 2), während der sterisch sehr anspruchsvolle tert.-Butyl-Substituent als $\mathrm{R}_{2}$ eine Isomerenbildung vollständig verhindern soll ${ }^{13}$. Bei dem Bis(tert.-butyl-methyl)ketazin kann nur das Isomer (C) isoliert werden. Schon bei Verringerung des sterischen Anspruchs des Substituenten $R_{2}$ durch Wahl einer Isopropyl-Gruppe treten zwei Isomere im Verhältnis C:B = 9:1 auf ${ }^{10}$. Für Ethyl als Substituent $R_{2}$ - also bei weiterer Reduktion des sterischen Anspruchs - treten alle Isomere auf ${ }^{10}$.

\subsection{Bor-Stickstoff-Verbindungen}

Die Bor-Stickstoff-Chemie erfuhr erst Anfang der 50er Jahre einen nennenswerten Aufschwung. Inzwischen sind neben Bor-Stickstoff-Addukten, den Amminboranen, auch Aminoborane ${ }^{14}$, Iminoborane ${ }^{15,16}$, und Borazine ${ }^{14}$ - kovalente und niederkoordinierte Bor-Stickstoff-Verbindungen - bekannt und weitgehend untersucht.

Da die BN-Gruppe isoelektronisch zu einer CC-Einheit ist, stimmen physikalische Eigenschaften und Strukturen von Verbindungen, die nur in dieser Struktureinheit voneinander abweichen, häufig überein. Die chemischen Eigenschaften weichen bei Kohlenstoff- und ihren BN-analogen Verbindungen allerdings deutlich voneinander ab.

Für diese Arbeit sind Ringsysteme von Interesse, die über intramolekulare Cyclisierungen gebildet werden. Dabei sind solche Ringschlüsse mit Elementen der 13. Hauptgruppe nicht neu ${ }^{17,18}$; im Arbeitskreis MELLER wurden ähnliche Lewis-SäureBase-Reaktionen mit Halogenboranen näher untersucht ${ }^{19}$. Auch mit Hydrazonen sind intramolekulare Ringschlüsse durchgeführt worden ${ }^{20}$. 


\subsubsection{Bor-Hydrazon-Verbindungen}

Bei der Umsetzung einfach $N$-alkylierter Hydrazone mit Silanen wurden im Arbeitskreis KLINGEBIEL Ringschlüsse beschrieben. Die Ergebnisse konnten auf Reaktionen mit Boranen übertragen werden ${ }^{21}$ :

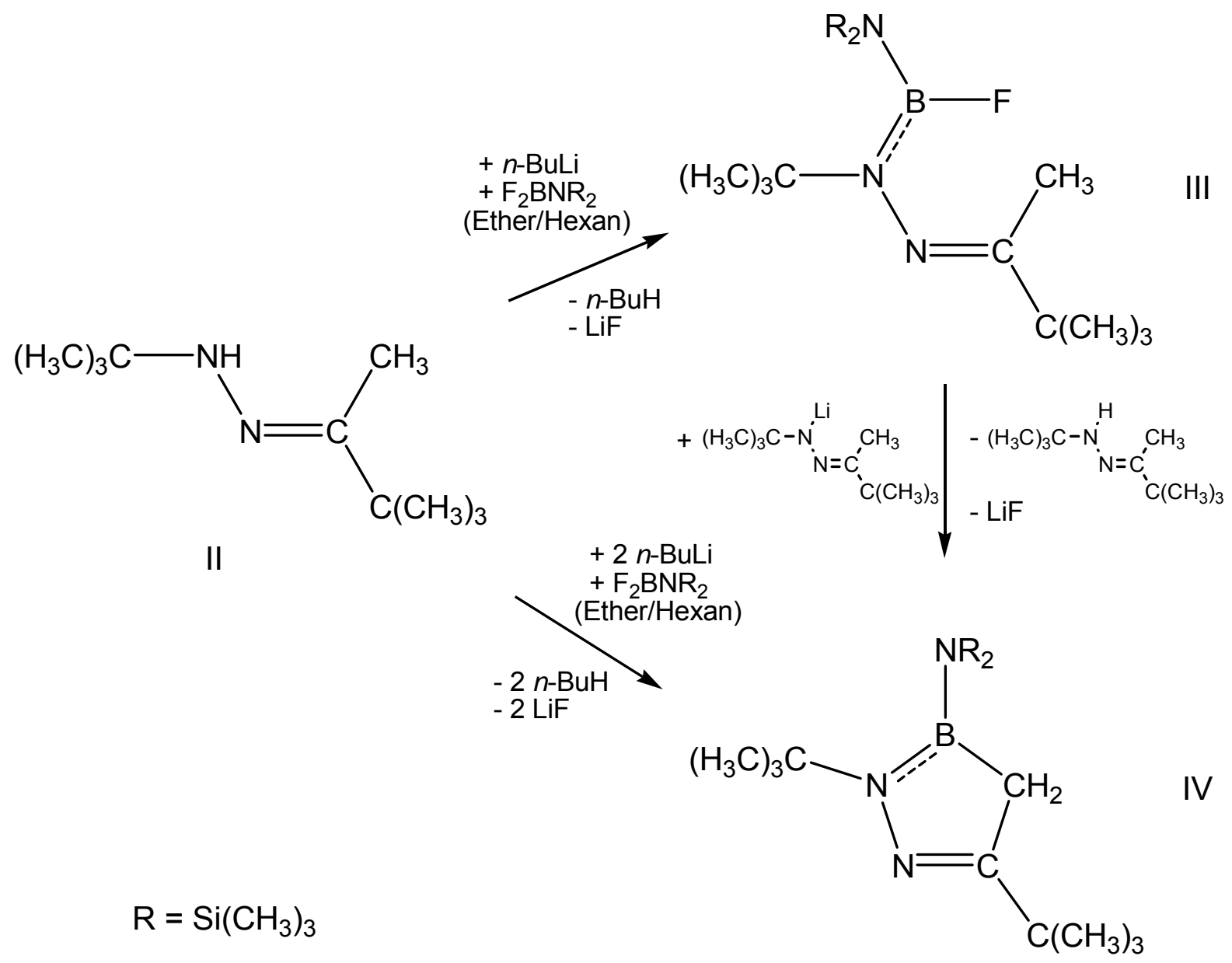

Schema 3: Intramolekulare Cyclisierungsreaktion eines Hydrazono-Aminoborans

Dass die Cyclisierungen - trotz Bildung eines fünfgliedrigen Rings - auch auf andere Weise erfolgen können, zeigten Ergebnisse aus dem Arbeitskreis MELLER ${ }^{22}$ : 
<smiles>[R]B(F)NN=C(C(C)C)C(C)C</smiles>

V

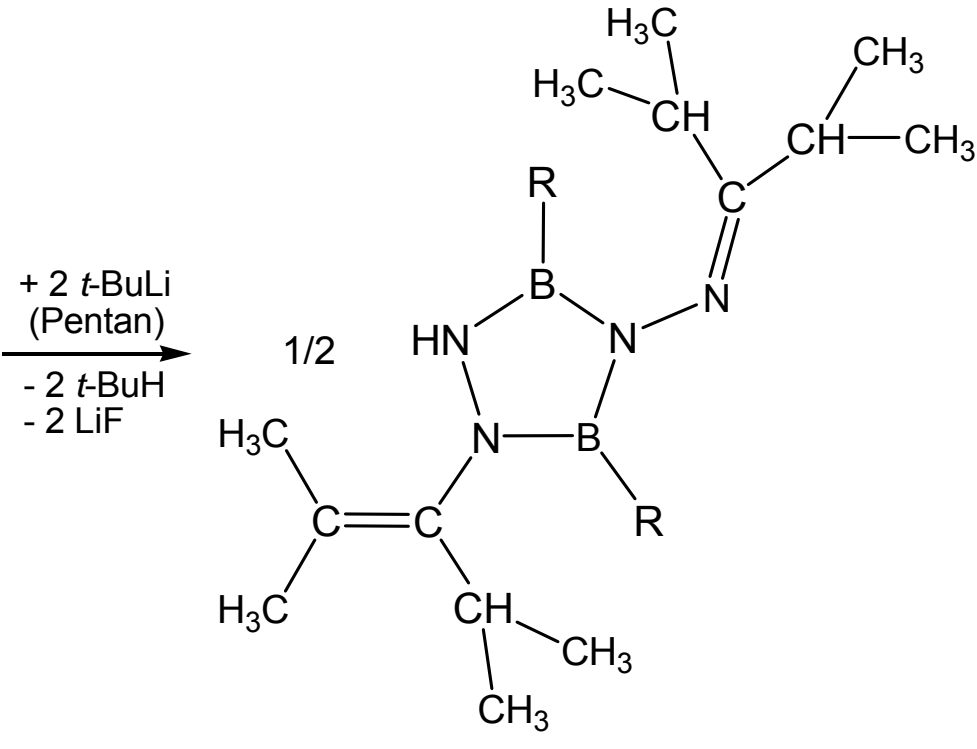

VI

Schema 4: Intramolekulare Cyclisierung eines Hydrazono-Arylborans

Bei der versuchten Darstellung von 1,2-Diaza-3-boracyclopentenen aus $N$-unsubstituierten Hydrazonen und Halogenboranen wurden teilweise ungewöhnliche $\mathrm{Er}$ gebnisse gefunden; statt eines $\mathrm{NH}-$ funktionellen Cyclopentens entstand ein $\mathrm{N}-(2-$ Butyliden)-substituierter fünfgliedriger Ring ${ }^{20}$ :<smiles>N[AlH]</smiles><smiles>N[IH]</smiles><smiles>C=NCC</smiles><smiles>C=C(C)C</smiles>

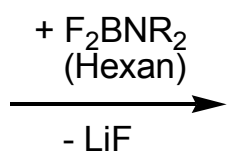<smiles>CC(C)(C)C</smiles>

$$
\mathrm{R}=\mathrm{CH}\left(\mathrm{CH}_{3}\right)_{3}
$$<smiles>C=C(CC(C)(C)C)C(C)(C)C</smiles><smiles>[R2][PH]1=NN=C(C(C)(C)C)C1</smiles>

Schema 5: Reaktion eines Hydrazons mit einem Halogenboran

Solche Ringe sollten bei der Verwendung von Ketazinen als Primärprodukt entstehen. Daraus resultierten weitere Untersuchungen zur Bor-Azin-Chemie. 


\subsubsection{Bor-Azin-Verbindungen}

Bei der Umsetzung von monolithiierten symmetrischen Azinen mit einer äquimolaren Menge an Halogenboranen bilden sich bevorzugt über intramolekulare Cyclisierung geschlossene fünfgliedrige Ringe - 1,2-Azaazonia-3-boratacyclopentene. Ein Stickstoffatom und das Boratom des Rings sind dabei vierfach koordiniert. Durch Variation der Menge des eingesetzten Halogenborans lassen sich auch größere Ringsysteme darstellen ${ }^{6,7}$ :

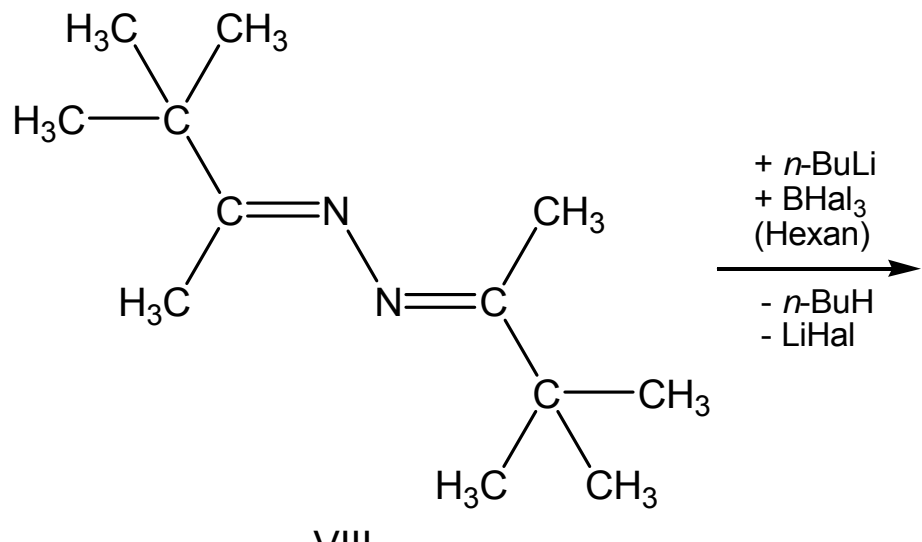<smiles></smiles><smiles>C/C(=N\N=C(/C)C(C)(C)C)C(C)(C)C</smiles>

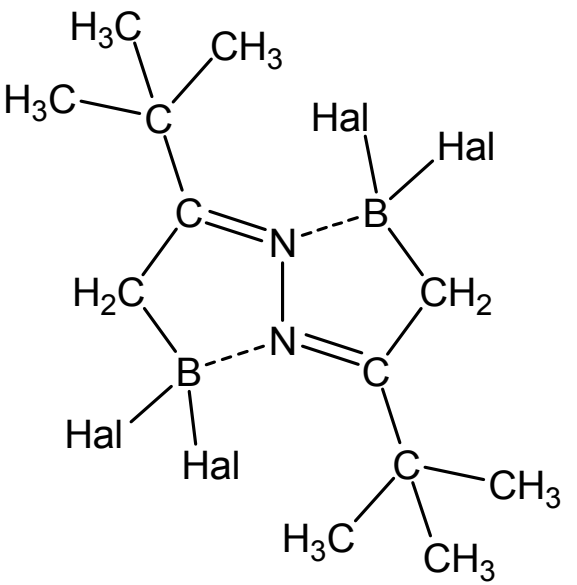

VIII

Schema 6: Übersicht über Reaktionen eines Azins mit Trihalogenboranen

Die eingeführten Halogenfunktionen lassen zahlreiche weiterführende Reaktionen zu. Substitutionsreaktionen mit Aryl-, Alkyl-, Alkoxi- und Aminosubstituenten sind durchgeführt worden ${ }^{6,7,23}$. Kopplungsreaktionen zu größeren Ringsystemen sind jedoch bislang genauso wenig untersucht wie Eigenschaften in Übergangsmetallkomplexen. 
Durch Dehydrohalogenierung lassen sich stabile cyclische Aminoborane erzeugen, die 1,2-Diaza-3-boracyclopentene. Eine durch die Dehydrohalogenierung provozierte Bildung einer $\mathrm{B}=\mathrm{C}$-Doppelbindung wird nicht beobachtet:

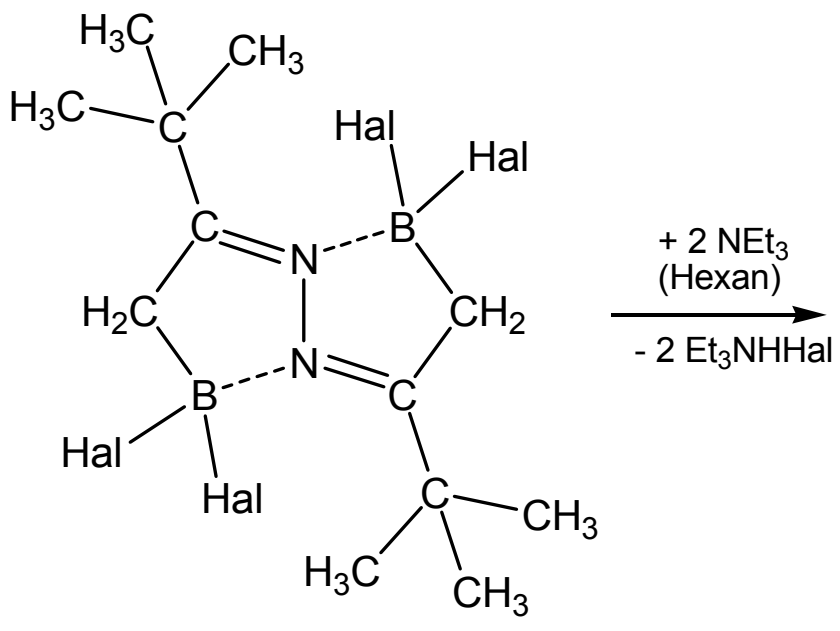<smiles></smiles>

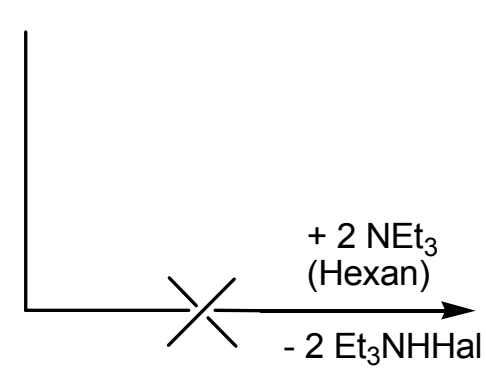<smiles></smiles>

Schema 7: Beispiel einer Dehydrohalogenierung einer Azin-Bor-Verbindung

Der Schwerpunkt bisheriger Untersuchungen lag einerseits auf der Erforschung des Einflusses der Liganden am Azin und an den Boranen und andererseits auf der Untersuchung der Cyclisierungsreaktion zu verschiedenen Ringsystemen. 


\subsection{Phosphorheterocyclen}

Phosphorheterocyclen sind in nahezu unüberschaubarer Anzahl bekannt und im allgemeinen gut untersucht. Einige Fachbücher zwischen 1989 und $2001{ }^{24,25,26}$ und ein älterer Artikel ${ }^{27}$ bieten eine Übersicht zu diesem Themengebiet.

\subsubsection{Auswahl bekannter Typen von Phosphor-Heterocyclen}

In Phosphorheterocyclen wird neben Phosphor am häufigsten Stickstoff, Sauerstoff oder Schwefel eingeführt. Die Eigenschaften dieser Heterocyclen sind ebenso vielfältig wie ihre Anzahl.

Unten aufgeführt sind ausgewählte Phosphorcyclen mit einem Phosphoratom und zwei oder mehr weiteren Heteroatomen:

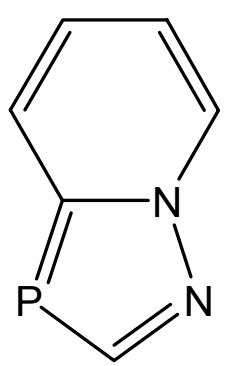

A

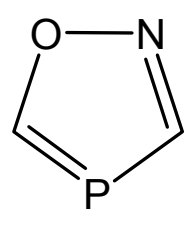

B

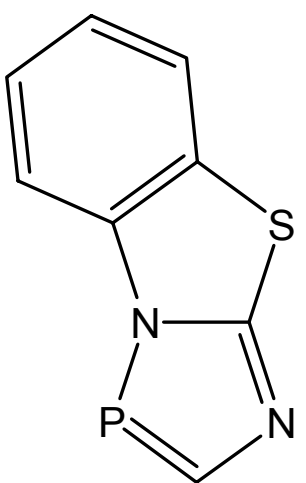

C

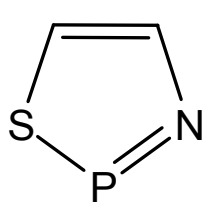

D

Abbildung 3: Auswahl bekannter Phosphorheterocyclen

Ein 1,2,4-Diazaphosphol (A), ein 1,2,4-Oxazaphosphol (B), ein 4H-1,4,2-Diazaphosphol und ein 1,3,2-Thiazaphosphol (D) stellen eine kleine Auswahl phosphorhaltiger Ringsysteme dar ${ }^{24}$.

Diese Arbeit beschäftigt sich mit der Synthese von fünfgliedrigen Ringen und achtgliedrigen Bicyclen aus Bis(tert.-butyl-methyl)ketazin. Die zu synthetisierenden Ringe enthalten demnach als Heteroelemente ein Phosphor- und zwei Stickstoffatome in jedem Ringelement. 


\subsubsection{Fünfgliedrige phosphor- und stickstoffhaltige Ringe}

Phosphor- und stickstoffhaltige fünfgliedrige Ringe (Azaphosphole (mit einem 6rSystem) oder Azaphosphacyclopentane) können nach Anzahl und Position der Phosphor- und Stickstoffatomen im Ring unterteilt werden. Für ein Phosphor- und zwei Stickstoffatome ergeben sich allein 4 Möglichkeiten der Anordnung ohne Berücksichtung von Substitutionen oder Mehrfachbindungen:

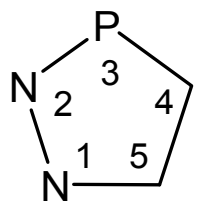

A

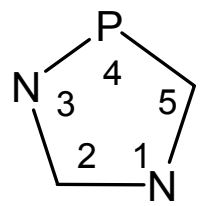

B

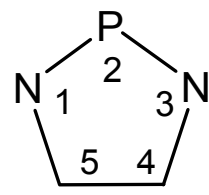

C

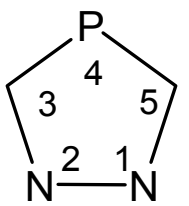

D

Abbildung 4: Mögliche Diazaphosphacyclopentane

Oben aufgeführt sind die (A) 1,2,3-, (B) 1,3,4-, (C) 1,3,2- und (D) 1,2,4-Diazaphosphacyclopentane bzw. Diazaphosphole ${ }^{24,25}$. Sie lassen sich nach Substitutionen an den Stickstoffatomen weiter untergliedern. Dies soll am Beispiel der 1,2,3-Diazaphosphole gezeigt werden:

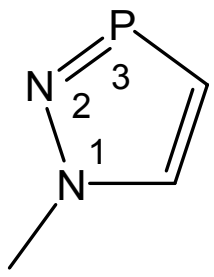

A

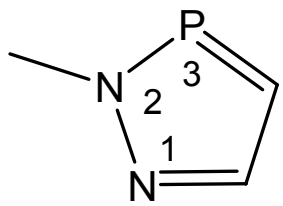

B

Abbildung 5: N1- und N2-substituierte 1,2,3-Diazaphosphole

Bei den abgebildeten Verbindungsklassen handelt es sich um die 1H-1,2,3-Diazaphosphole (A) und die 2H-1,2,3-Diazaphosphole (B). Die Umsetzung von Hydrazonen mit Phosphanen ergibt bis auf wenige Ausnahmen Typ $B^{24,25}$. 


\subsubsection{1,2-Diaza-3-phosphacyclopent-5-ene und -penta-3,5-diene}

1,2-Diaza-3-phosphacyclopent-5-ene (3,4-Dihydro-2H-1,2,3-diazaphosphole) und 1,2-Diaza-3-phosphacyclopenta-3,5-diene (2H-1,2,3-Diazaphosphole) sind bereits eingehend untersuchte und seit längerem bekannte Verbindungstypen ${ }^{28,29,30,31,32}$. Sie wurden bislang aus Hydrazinen oder Hydrazonen dargestellt. Die P-Alkylsubstituierten Cyclopentene sind hydrolyseempfindlich, wohingegen die Diene sehr stabil gegenüber Wasser, Luftsauerstoff oder elementarem Schwefel sind. Additionen am Phosphor- oder Kohlenstoffatom der $\mathrm{P}=\mathrm{C}$-Doppelbindung der Diene sind im allgemeinen möglich und z.B. mit Brom untersucht ${ }^{24,25}$.

Von besonderem Interesse ist die geringe Nukleophilie des Phosphors für Komplexe mit Übergangsmetallen. Röntgenstrukturen mit Koordination über das Phosphor- oder ein Stickstoffatom des Rings sind veröffentlicht ${ }^{33,34,35,36,37,38}$.

Die oben genannten Ringsysteme aus Ketazinen sind allerdings noch nicht in der Literatur erwähnt. Möglicherweise reagieren Phosphorverbindungen mit den Dianionen der Ketazine zu einem größeren Ring:

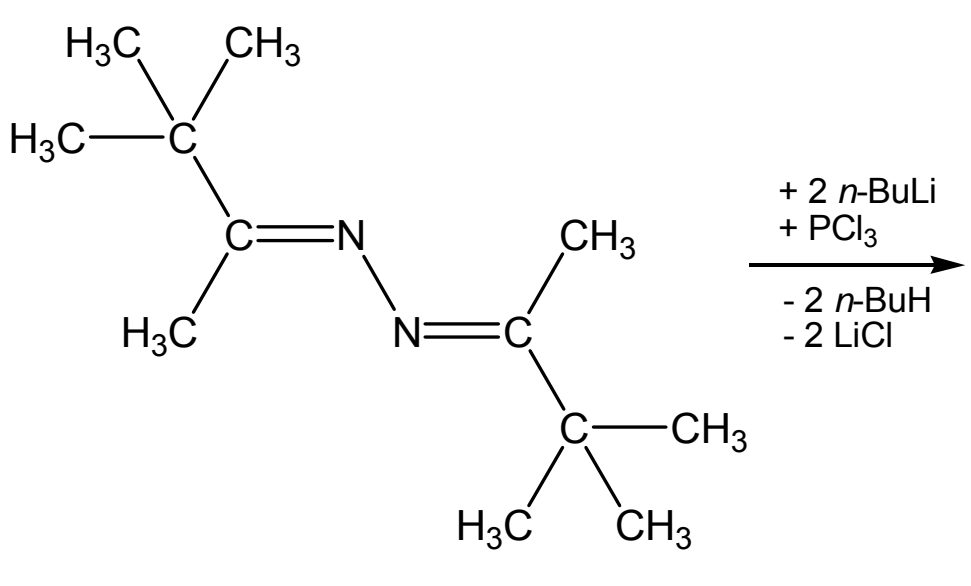

VIII

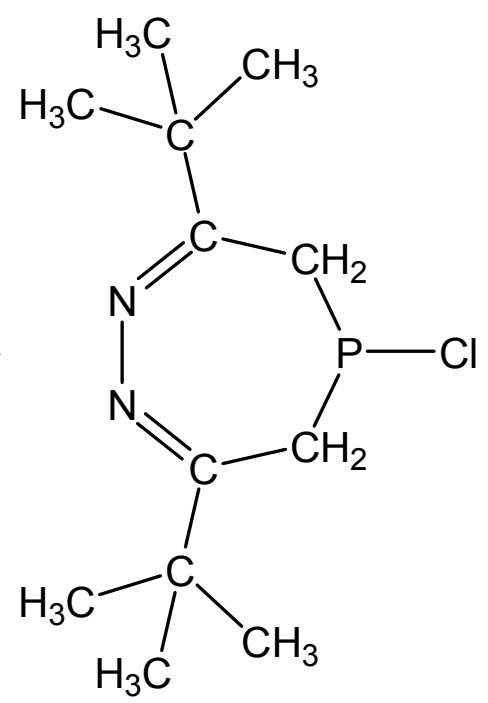

1

Schema 8: Mögliche Reaktion von Trichlorphosphan mit einem Ketazindianion 


\subsection{Cyclohexaphosphane}

Diese Klasse der cyclischen Phosphorverbindungen ist zwar seit den 60er Jahren bekannt ${ }^{39,40}$, doch wurde sie seitdem noch wenig untersucht. Bislang sind Phosphorsechsringe mit Wasserstoff- ${ }^{40,41}$, Aryl- ${ }^{42,43,44}$ oder Alkyl-Substituenten ${ }^{45,46}$ veröffentlicht; zum Teil sind auch die Eigenschaften und Bindungsarten als Ligand in Komplexen untersucht ${ }^{47,48,49}$. Cyclohexaphosphane sind unter den Cyclophosphanen selten und es sind dementsprechend wenige Daten bekannt. Ihre zumeist geringe Löslichkeit erschwert die Kernspinresonanzuntersuchungen. 


\section{Problemstellung}

Das Reaktionsverhalten der Bor-Azin-Verbindungen ist inzwischen in Grundzügen geklärt; zum Großteil stehen jedoch noch Strukturbeweise aus. Substitutionsreaktionen haben sich bislang auf die bicyclischen achtgliedrigen Ringe beschränkt. Azaazoniaboratacyclopentene können in solche Bicyclen überführt werden ${ }^{6}$. Sie stellen aber keineswegs nur Zwischenprodukte dar ${ }^{7}$.

Ein Schwerpunkt dieser Arbeit liegt auf der Untersuchung des Reaktionsverhaltens dieser borhaltigen fünfgliedrigen Ringe; insbesondere sollen Halogensubstitutionen und Dehydrohalogenierungen erforscht werden, die schon erfolgreich an den bicyclischen Verbindungen durchgeführt werden konnten.

Umsetzungen der Azine mit Heteroatomverbindungen (außer Borverbindungen) sind noch weitgehend unerforscht. Es liegt nahe, zunächst weitere Reaktionen mit Hauptgruppenelementverbindungen zu untersuchen. Im Arbeitskreis KLINGEBIEL wurden mit einigem Erfolg Silane mit Hydrazinen ${ }^{50,51,52}$ und Hydrazonen 21,53,54,55 umgesetzt. Ähnlich vielversprechend sind Phosphor(III)verbindungen, bei denen eine starke Tendenz zur Ausbildung von fünfgliedrigen Ringsystemen bekannt ist 24,25,26,27. Diese sind bisher jedoch fast ausschließlich aus Hydrazonen oder Hydrazinen dargestellt worden.

Ein zweiter Schwerpunkt dieser Arbeit liegt auf der Untersuchung der Übertragbarkeit von Ergebnissen der Phosphor-Hydrazon- und der Bor-Azin-Chemie auf die Phosphor-Azin-Chemie.

Syntheserouten und Steuerung von Produktbildung durch Variation von Reaktionsbedingungen sollten der Phosphor-Hydrazon-Chemie ähneln. Dazu werden einige Reaktionen durchgeführt und ausgewertet. Eine Produktbildung über Bor-Azin-Chemie analoge Darstellungsmethoden ist weniger wahrscheinlich. Dort findet eine intramolekulare Cyclisierung über eine $\mathrm{N} \rightarrow \mathrm{B}$-Adduktbildung statt. Dies ist bei einer analogen Phosphorverbindung nicht möglich. Die Reaktionsführung sollte sich deshalb deutlich unterscheiden. 


\section{Ergebnisse und Diskussion}

Im Ergebnisteil abgebildete Röntgenstrukturen sind Stick-and-Ball-Abbildungen. Dabei sind Einfachbindungen grau, Doppelbindungen weiß gefärbt und Adduktbindungen gestrichelt dargestellt. Die Ortep-Plots mit anisotropen Auslenkungsparametern mit 50 \%iger Wahrscheinlichkeit sind in Kapitel 8.2 angegeben.

\subsection{Bor-Azin-Chemie}

Obwohl Reaktionen von Boranen mit Azinen inzwischen recht ausführlich untersucht wurden, bleiben doch einige Punkte dieser Chemie - vor allem mechanistische und theoretische Aspekte - ungeklärt. Die Synthese und Charakterisierung weiterer Verbindungen soll hier zur Aufklärung beitragen.

\subsubsection{Kristallstruktur des disilylierten Ketazins IX}

Erfahrungen mit Chlor- und Fluorboranen haben gezeigt, dass ein Trimethylsilylsubstituent zusammen mit einem Halogen eine gute Abgangsgruppe ist, um BC-Bindungen zu knüpfen. Dazu wurden in der Arbeitsgruppe MELLER mono- ${ }^{8}$ und disilylierte Ketazine ${ }^{7,8}$ dargestellt und mit Halogenboranen zur Reaktion gebracht. Es liegt nahe, solche Silylketazine auch in der Umsetzung mit Phosphorverbindungen einzusetzen. Die Synthese von IX erfolgte gemäß Literaturvorschrift ${ }^{7}$ :

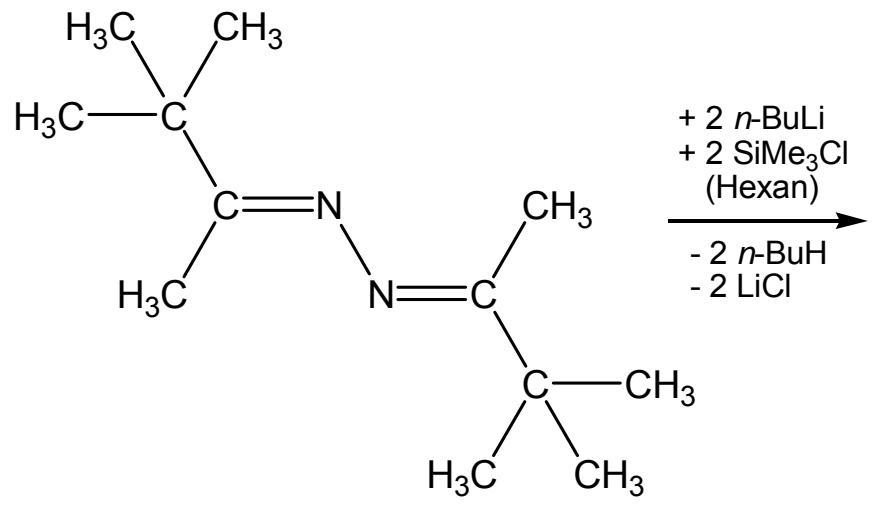

VIII<smiles>CC(C)(C)/C(C[Si](C)(C)C)=N/N=C(\C[Si](C)(C)C)C(C)(C)C</smiles>

IX

Schema 9: Darstellung des Bis(tert.-butyl-(trimethylsilyl)methyl)ketazins IX 
Bei der erneuten Darstellung des Bis(tert.-butyl-(trimethylsilyl)methyl)ketazins IX gelang durch Umkristallisation aus $n$-Hexan die Bildung von röntgenstrukturtauglichen Einkristallen:

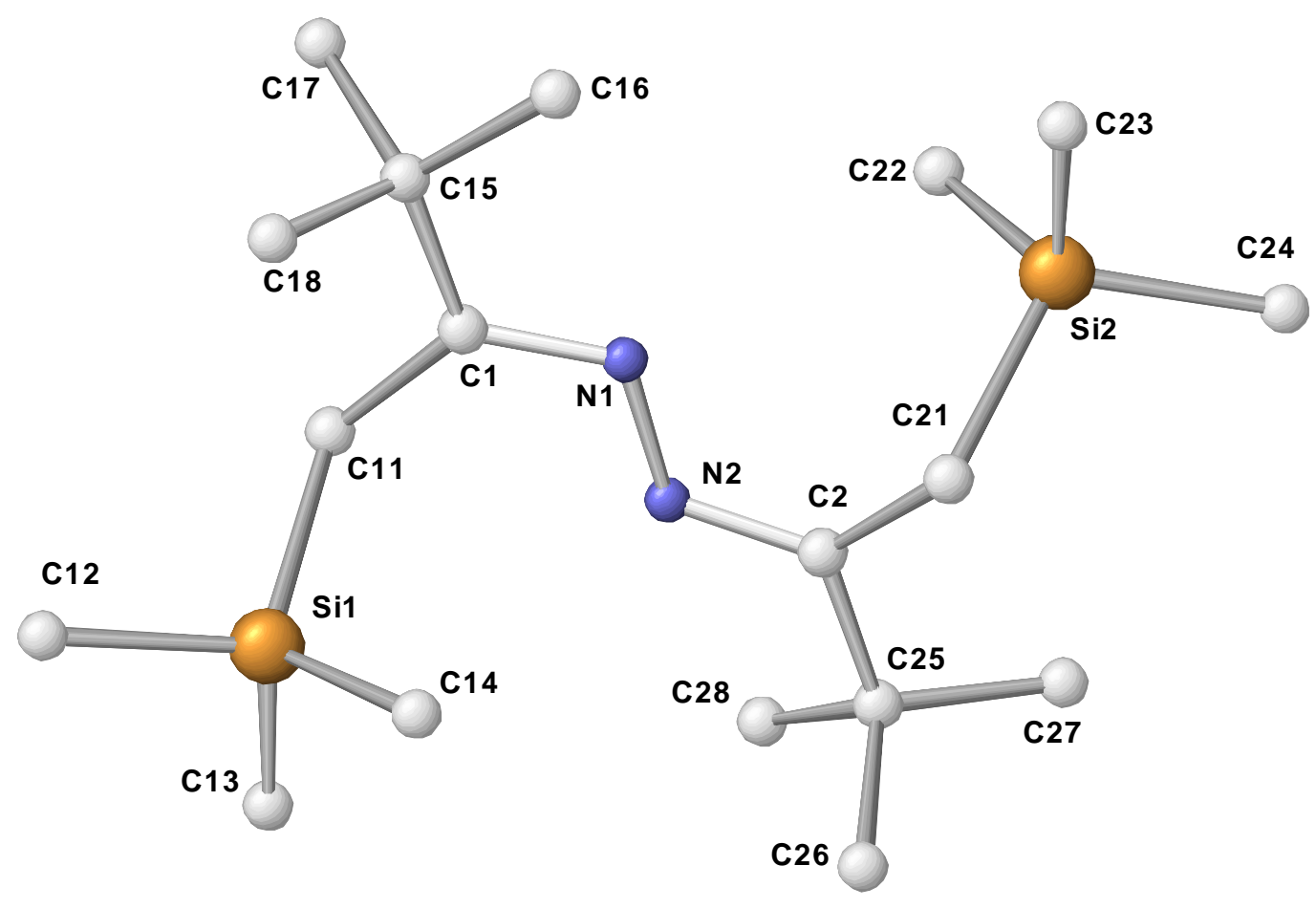

Abbildung 6: Struktur von Verbindung IX (ohne Wasserstoffatome)

Verbindung IX kristallisiert in einem monoklinen System mit der Raumgruppe P2(1)/c. Deutlich bleibt das Ketazingrundgerüst bis auf eine leichte Torsion gegenüber dem unsilylierten Azin VIII unverändert. Die CNN-Winkel sind mit $113^{\circ}$ um $7^{\circ}$ kleiner als der ideale Winkel einer trigonal-planaren Konfiguration der Stickstoffatome. Dies lässt sich auf die freien Elektronenpaare an den Stickstoffatomen zurückführen. Die Bindungslängen $\mathrm{C}(1)-\mathrm{N}(1)$ und $\mathrm{C}(2)-\mathrm{N}(2)$ betragen je $128 \mathrm{pm}$ und weisen damit einen typischen $\mathrm{C}=\mathrm{N}$-Doppelbindungscharakter auf ${ }^{16}$. Die Siliziumatome befinden sich nicht in direkter Nähe zu den Stickstoffatomen; obwohl eine stabilisierende Koordination der elektropositiven Siliziumatome an die Stickstoffatome, bzw. an deren freie Elektronenpaare, denkbar wäre.

Aus der Struktur von Verbindung IX lassen sich Rückschlüsse auf das Dilithiumsalz des Ketazins VIII ziehen: Die Lithiumionen sind vermutlich nicht - wie man auf Grund der Elektronegativitäten und der geringen Polarisierbarkeit des Lithiums und des 
Stickstoffs erwarten kann - an die Stickstoffatome gebunden. Vielmehr scheint das $\alpha-$ Kohlenstoffatom durch die Deprotonierung zu einer für das Lithium ausreichend harten Lewisbase zu werden. Das Lithiumorganyl könnte sich durch eine räumliche Ausrichtung des Lithiums zum freien Elektronenpaar des Stickstoffs stabilisieren. Eine Lewisstrukturformel sieht vermutlich wie folgt aus:

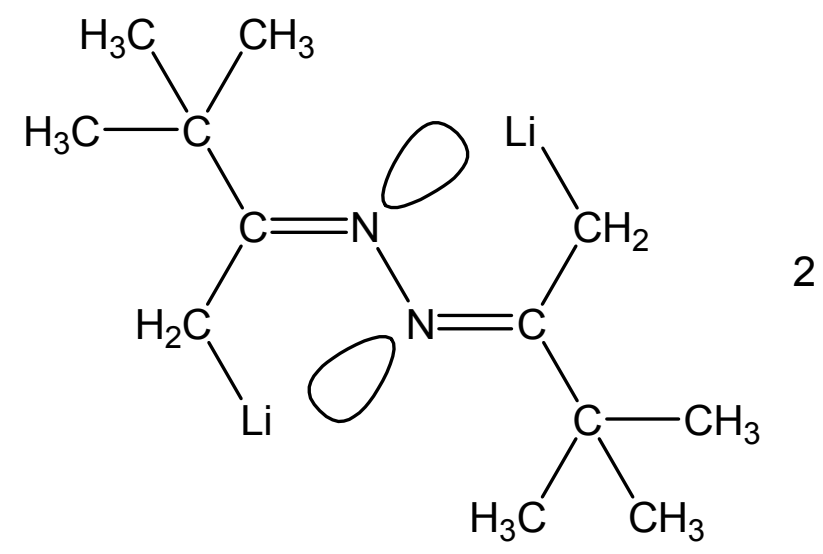

Abbildung 7: Strukturvorschlag für das Dilithiumsalz 2 des Bis(tert.-butyl-methyl)ketazins

Dafür sprechen auch die Röntgenstrukturen der bicyclischen Bor-Azin-Verbindungen aus dem Arbeitskreis MELLER ${ }^{6,7}$. Diese lassen sich durch einfachen Austausch des Lithiums durch einen Borylrest formulieren:<smiles></smiles>

2

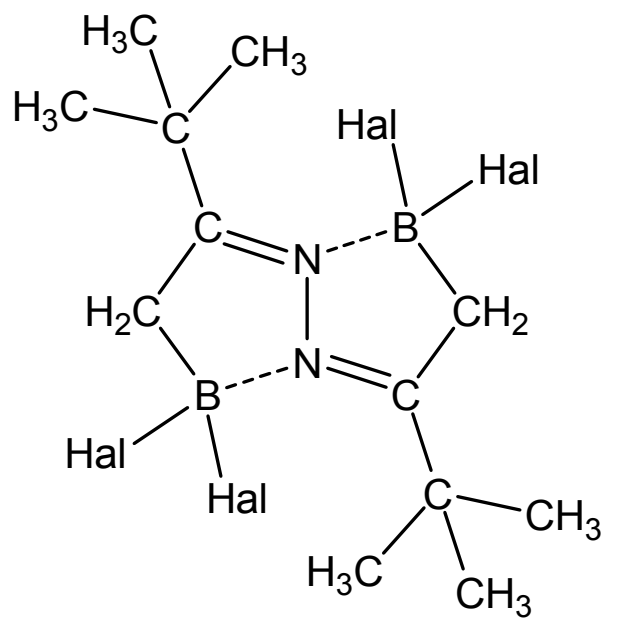

Abbildung 8: Vergleich des Dilithiumsalzes 2 mit einer bicyclischen Bor-Azin-Verbindung 
Bei einer Positionierung des Lithiums am Stickstoff wäre die sofortige Ausbildung eines Aminoborans ohne Cyclisierung wahrscheinlich, wie sie z.T. bei Hydrazonen beobachtet wird (vgl. Schema 3).

Die Röntgenstrukturdaten der in Abbildung 8 dargestellten Bicyclen mit Hal = Fluor, Chlor, Brom und Jod weisen mit BN-Bindungslängen von 158 pm - 166 pm auf ein Amminboran hin. Für eine B-N-Einfachbindung gibt PAETZOLD eine mittlere Bindungslänge von $159 \mathrm{pm}$ an ${ }^{16}$. Auch die ${ }^{11} \mathrm{~B}-\mathrm{NMR}$-Signale des Bors weisen durch Schärfe und Verschiebung (+6 bis -34 ppm) auf eine vierfache Koordination hin ${ }^{6}$. Dies spricht dafür, dass zunächst die BC-Bindung geknüpft wird und anschließend eine intramolekulare Cyclisierung über eine $\mathrm{N} \rightarrow \mathrm{B}$-Adduktbildung erfolgt (wie von MELLER vorgeschlagen ${ }^{6,7,23}$ ).

\subsubsection{Synthese weiterer fünfgliedriger borhaltiger Ringe}

Nicht ganz eindeutig lässt sich der Reaktionsmechanismus für die Umsetzung des zweifach metallierten Ketazins mit einem Äquivalent Halogenboran erklären. Die sterischen Aspekte sprechen für die Ausbildung eines siebengliedrigen Ringsystems (vgl. vorgeschlagene Reaktion für $\mathrm{PCl}_{3}$ mit einem Ketazin in Schema 8). Tatsächlich bildet sich ein fünfgliedriger Ring mit einem konjugierten Elektronenpaarsystem $(\mathrm{C}=\mathrm{C}-\mathrm{N}-\mathrm{N}=\mathrm{C})$, bei dem eine exocyclische und eine endocyclische Doppelbindung über das freie Elektronenpaar des Stickstoffatoms miteinander in Verbindung stehen. Die Synthese weiterer borhaltiger fünfgliedriger Ringe liefert neues Datenmaterial und damit neue Ansätze zur Diskussion über diesen Reaktionsverlauf.

\subsubsection{Kristallstruktur des 5-tert.-Butyl-2-(3,3-dimethylbutyliden-2)-3,3-dichlor- 1,2-azaazonia-3-boratacyclopent-5-ens $X$}

In den von MELLER ${ }^{6,7}$ dargestellten Reaktionen spielen die Azaazoniaboratacyclopentene als Zwischen- und Nebenprodukte bei der Bicyclisierung eine Rolle. Substitutionen am 5-tert.-Butyl-2-(3,3-dimethylbutyliden-2)-3,3-dichlor-1,2-azaazonia-3boratacyclopent-5-en $(\mathbf{X})$ sind bislang nicht bekannt. Die Darstellung von $\mathbf{X}$ erfolgte in Anlehnung an SCHMIDPETER ${ }^{24,25}$ durch Zugabe von einem Äquivalent Trichlorboran 
zu einer Lösung aus Ketazin und Toluol. Durch Erhitzen wurde entstehendes Chlorwasserstoffgas ausgetrieben:<smiles>C/C(=N\N=C(/C)C(C)(C)C)C(C)(C)C</smiles>

VIII<smiles></smiles>

$\mathrm{X}$

Schema 10: Darstellung von Verbindung $\mathbf{X}$

Bei der Darstellung des 5-tert.-Butyl-2-(3,3-dimethylbutyliden-2)-3,3-dichlor-1,2azaazonia-3-boratacyclopent-5-ens $\mathbf{X}$ gelang es durch Umkristallisation aus $n$-Hexan röntgenfähige Einkristalle zu erhalten.

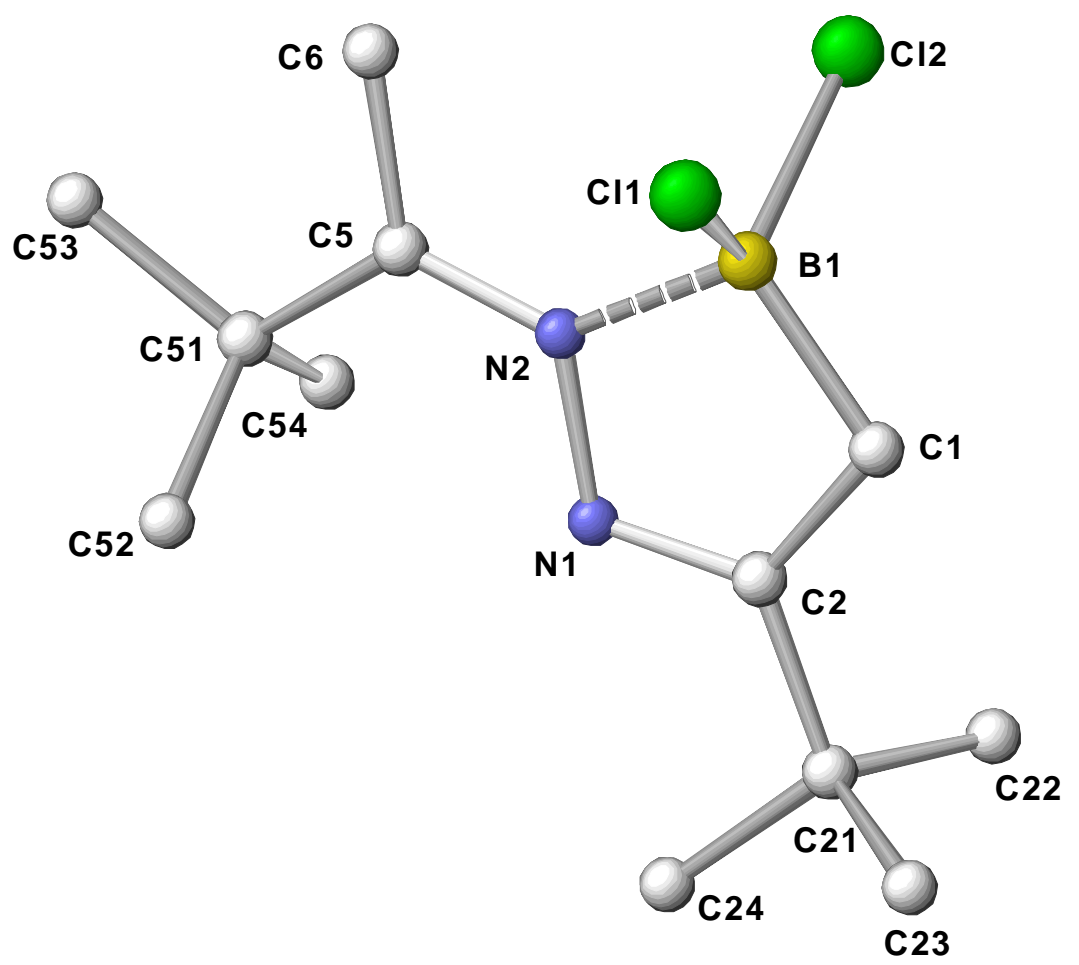

Abbildung 9: Struktur von Verbindung $\mathbf{X}$ (ohne Wasserstoffatome) 


\begin{tabular}{|ll||cc|}
$\mathrm{N}(1)-\mathrm{N}(2)$ & 142,4 \\
$\mathrm{~N}(2)-\mathrm{B}(1)$ & 160,9 \\
$\mathrm{~B}(1)-\mathrm{C}(1)$ & 159,4 \\
$\mathrm{C}(1)-\mathrm{C}(2)$ & 149,3 \\
$\mathrm{C}(2)-\mathrm{N}(1)$ & 127,9 \\
$\mathrm{C}(5)-\mathrm{N}(2)$ & 129,1 & $\mathrm{~N}(1)-\mathrm{N}(2)-\mathrm{B}(1)$ & 109,9 \\
$\mathrm{~N}(2)-\mathrm{B}(1)-\mathrm{C}(1)$ & 99,7 \\
$\mathrm{~B}(1)-\mathrm{C}(1)-\mathrm{C}(2)$ & 103,0 \\
$\mathrm{C}(1)-\mathrm{C}(2)-\mathrm{N}(1)$ & 117,7 \\
$\mathrm{C}(2)-\mathrm{N}(1)-\mathrm{N}(2)$ & 109,5 \\
& $\Sigma$ Innenwinkel & 539,8
\end{tabular}

Tabelle 1: Ausgewählte Bindungslängen $(\mathrm{pm})$ und -winkel $\left({ }^{\circ}\right)$ von Verbindung $\mathbf{X}$

Die Verbindung $\mathbf{X}$ bildet ein monoklines Kristallsystem mit der Raumgruppe P2(1)/C aus. Nach der Formel für ein gleichseitiges Vieleck - $180^{\circ} \cdot(n-2)=$ Innenwinkelsumme (mit $\mathrm{n}=$ Anzahl der Atome im Ring) - muss ein planarer fünfgliedriger Ring eine Innenwinkelsumme von $540^{\circ}$ aufweisen. Die Summe der Innenwinkel im Ring beträgt $539,8^{\circ}$; der Ring ist demnach planar, wie die nachfolgende Abbildung bestätigt:

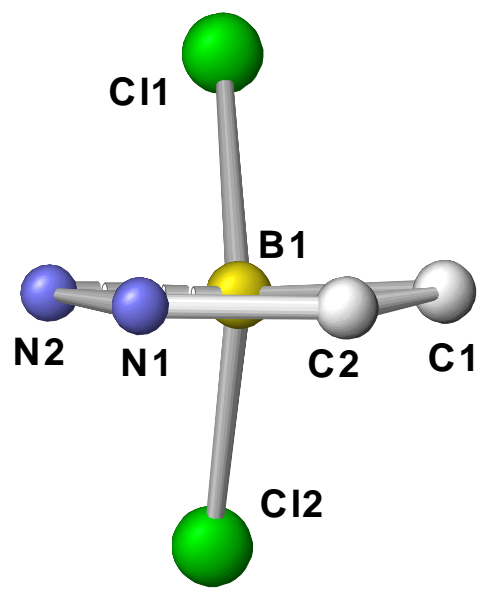

Abbildung 10: Seitenansicht der Ringebene von Verbindung $\mathbf{X}$ (ohne Alkylsubstituenten an $\mathrm{N}(2)$ und $\mathrm{C}(2)$ )

Die Winkelsumme an $\mathrm{N}(2)$ beträgt $359,9^{\circ}$. Das Stickstoffatom ist demnach $\mathrm{sp}^{2}-$ hybridisiert und trigonal-planar. Für $\mathrm{N}(1)$ ist nur der Winkel $\mathrm{N}(2)-\mathrm{N}(1)-\mathrm{C}(2)$ messbar, der mit $109,5^{\circ}$ einem Innenwinkel eines regelmäßigen fünfgliedrigen Ringes nahe kommt $\left(5 \cdot 108^{\circ}=540^{\circ}\right)$. Zudem sollte dieser Winkel durch das freie Elektronenpaar an $\mathrm{N}(1)$ im Vergleich zu einem streng trigonal-planar ausgerichteten $120^{\circ}$-Winkel verkleinert sein. Dies spricht ebenfalls für die $\mathrm{sp}^{2}$-Hybridisierung.

Der fünfgliedrige Ring ist damit mit den einzelnen Ringen der schon bekannten Bicyclen aus dem Arbeitskreis MELLER vergleichbar ${ }^{6,7,8}$. Dort sind zwei planare fünf- 
gliedrige Ringe über die beiden Stickstoffatome ohne axiale Verdrehung zu einem symmetrischen planaren achtgliedrigen Ring verknüpft.<smiles></smiles>

$\mathbf{X}$<smiles></smiles>

XI

Abbildung 11: Vergleich von Verbindung $\mathbf{X}$ mit dem analogen Bicyclus XI

Einzige Ausnahme bildet das 4,8-Di-tert.-butyl-2,2,6,6-tetrajod-1,5-diaza-2,6-diborabicyclo[3.3.0]octa-3,7-dien, bei dem die beiden Boratome aus den Ebenen der fünfgliedrigen Ringen herausstehen. Mit den Daten der analogen Dichlorborylverbindung XI stimmen Innenwinkelsumme und Bindungslängen im Rahmen der angegeben Fehlergrenzen nahezu vollständig überein. Der BN-Abstand entspricht wieder mit rund 161 pm einer $\mathrm{B}-\mathrm{N}-$-Einfachbindung. Ebenso liegen typische $\mathrm{C}=\mathrm{N}-$ Doppelbindungen mit 127,9 bzw. 129,1 pm vor (s.o.). Auch die NMR-Daten gleichen sich weitgehend.

\subsubsection{Substitution von 5-tert.-Butyl-2-(3,3-dimethylbutyliden-2)-3,3-dichlor- 1,2-azaazonia-3-boratacyclopent-5-en}

Da Substitutionen bislang nur an Diazaboracyclopentenen erfolgten, sind B-Alkyl-BHalogen- oder $B$-Aryl-B-Halogen-Azaazoniaboratacyclopentene nicht bekannt. Solche Verbindungen sollten durch Substitution von $\mathbf{X}$ ebenfalls über die Umsetzung von monolithiierten Ketazinen mit Alkyl- oder Aryldihalogenboranen zugänglich sein. 
Während die Umsetzung von Verbindung $\mathbf{X}$ mit Methyllithium in Ether zu keinen isolierbaren Produkten führte, war die Reaktion mit tert.-Butyllithium in $n$-Hexan erfolgreich:
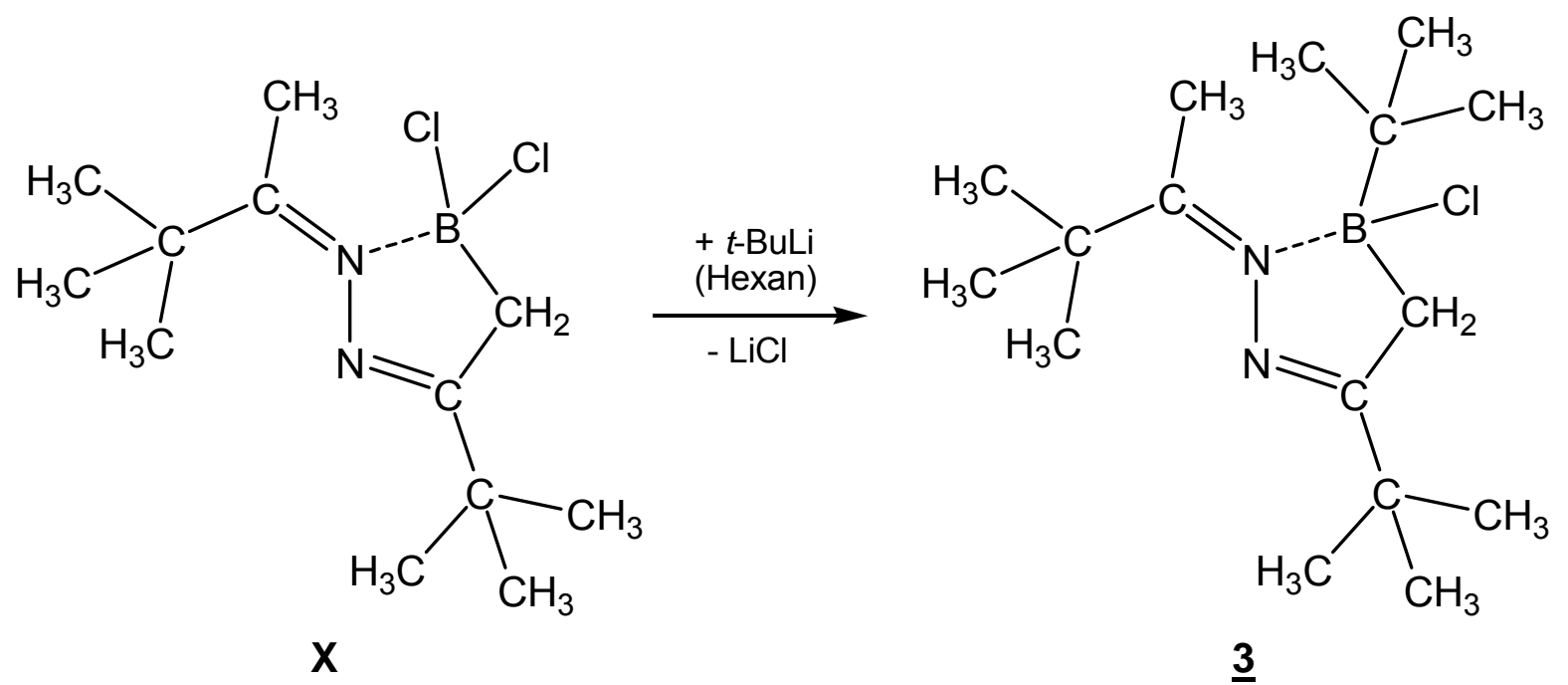

Schema 11: Reaktion des Azaazoniacyclopentens X mit tert.-Butyllithium

Wegen der geringen Massenunterschiede der Verbindungen ist die destillative Trennung aufwendig; sie reduziert die Ausbeute des Reinprodukts auf ein Viertel der eingesetzten Stoffmenge. Eine Aufarbeitung durch Extraktion ist aber wegen des ähnlichen Löseverhaltens keine Alternative.

Das Azaazoniacyclopenten $\underline{\mathbf{3}}$ ist wie $\mathbf{X}$ ein gelber Feststoff. Durch Umkristallisation aus $n$-Hexan bzw. Dichlormethan werden keine Einkristalle gewonnen. Es bildet sich vielmehr ein mikrokristalliner Niederschlag. Unter Feuchtigkeitsausschluss sind Azaazoniacyclopentene bei Raumtemperatur mehrere Wochen stabil.

\subsubsection{Dehydrohalogenierung von 5-tert.-Butyl-2-(3,3-dimethylbutyliden-2)-3,3- dichlor-1,2-azaazonia-3-boratacyclopent-5-en}

Die Darstellung von Verbindung $\mathbf{X}$ in Anlehnung an SCHMIDPETER ${ }^{24,25}$ zeigt, dass eine Dehydrohalogenierung möglich ist. Zur Abspaltung eines zweiten Äquivalents Chlorwasserstoff reicht weiteres Erhitzen von $\mathbf{X}$ in Benzol oder Toluol nicht aus. Durch Zugabe von 2 Äquivalenten Triethylamin (100 \%iger Überschuss) wird eine Dehydrohalogenierung teilweise erreicht: 

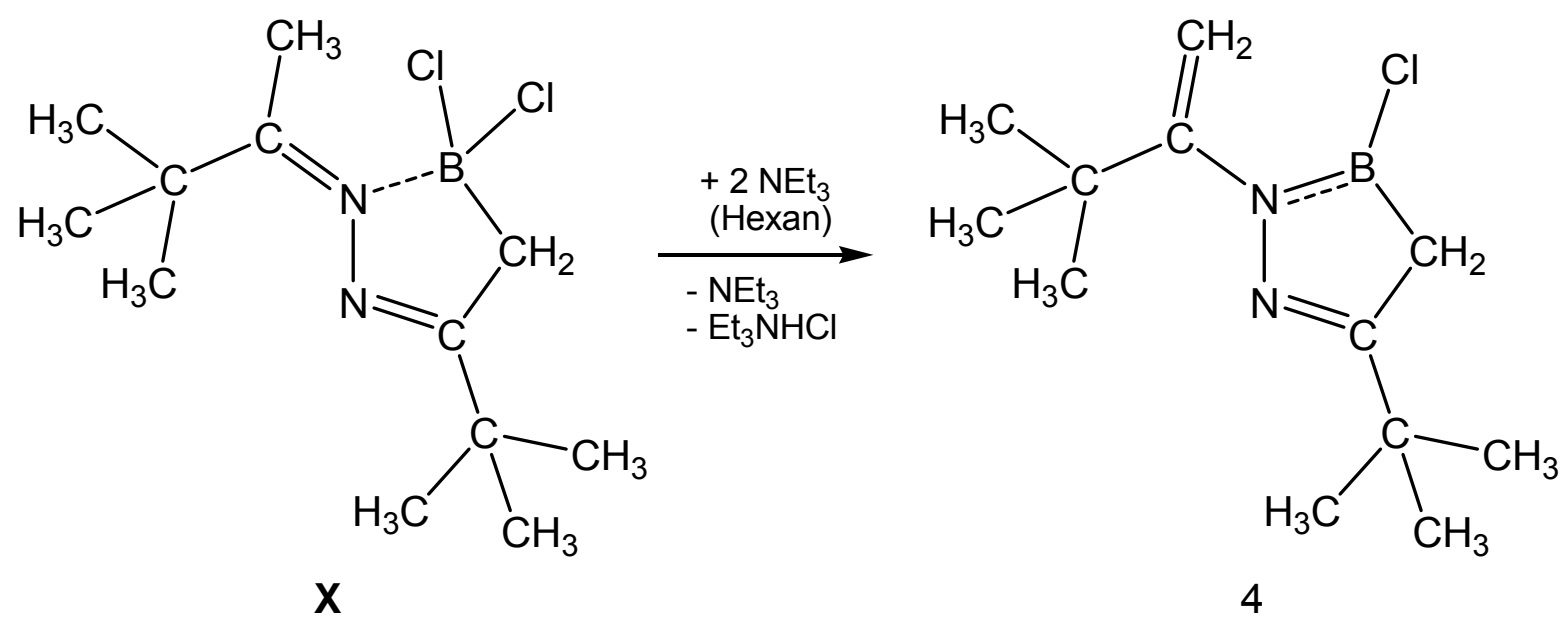

Schema 12: Dehydrohalogenierung von Verbindung $\mathbf{X}$

Nach dem Abtrennen des Triethylaminhydrochlorids lässt sich destillativ nur ein Gemisch aus $\mathbf{X}$ und 4 erhalten. Eine Trennung ist durch Extraktion mit $n$-Hexan in einer Soxhlet-Apparatur möglich. Das Diazaboracyclopenten 4 ist ein gelber Feststoff.

Eine eindeutige Unterscheidung der beiden Verbindungen kann durch ${ }^{11} \mathrm{~B}-\mathrm{NMR}$ Messungen erfolgen. Während Verbindung $\mathbf{X}$ ein - für ein vierfach koordiniertes Boratom typisches - scharfes Signal bei 7,4 ppm liefert, ist das Signal von Verbindung 4 breit und bei 27,0 ppm zu finden.

Diazaboracyclopentene mit einem monohalogensubstituierten Boratom waren bislang nur über Lithiumsalze silylierter Ketazine zugänglich ${ }^{7,23}$. Derartige Ringe sind interessante Ausgangsstoffe für einen weiteren Dehydrohalogenierungsschritt oder beliebige Substitutionsreaktionen.

Um eine vollständigere Dehydrohalogenierung zu erzielen, versetzt man Verbindung $\mathbf{X}$ mit einem vierfachen Überschuss an Triethylamin. Dabei kann folgendes Produkt isoliert werden: 


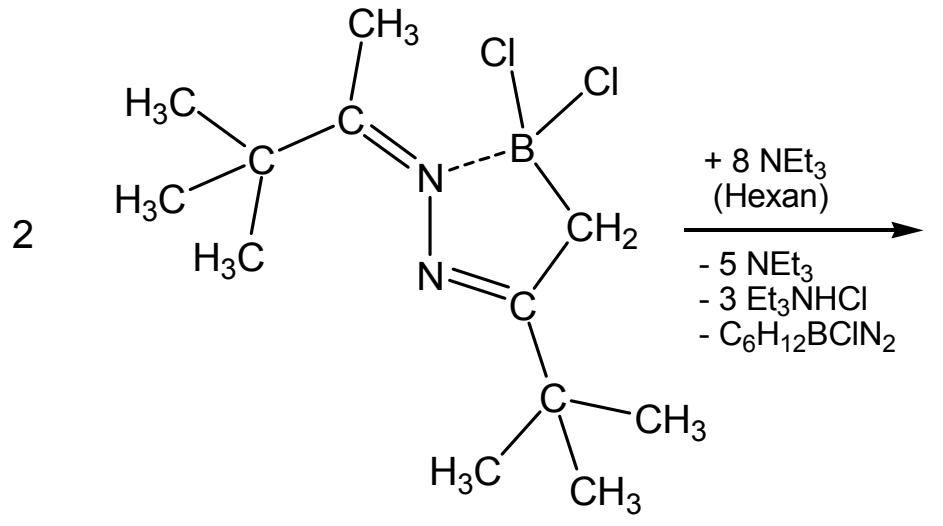

$\mathbf{X}$<smiles>CC(C)(C)C1=CB2CC(C(C)(C)C)=C(C(C)(C)C)N2N=C1C(C)(C)C</smiles>

$\underline{5}$

Schema 13: Dehydrohalogenierung von Verbindung $\mathbf{X}$

Verbindung $\underline{\mathbf{5}}$ kann mit der Eliminierung zweier Chlorwasserstoffmoleküle und der Spaltung einer CN-Bindung unter den drastischen Bedingungen erklärt werden (großer Amin-Überschuss, deutlich basisches Milieu, Siedetemperatur des Hexans). Ein möglicher Reaktionsweg ist in Schema 4 vorgestellt. Danach könnte durch Abspaltung eines zweiten Chlorwasserstoffs aus der ersten Dehydrohalogenierungsstufe 4 intermediär eine $B=C$-Doppelbindung entstehen, die sich über einen Angriff auf ein zweites Molekül 4 stabilisiert. 


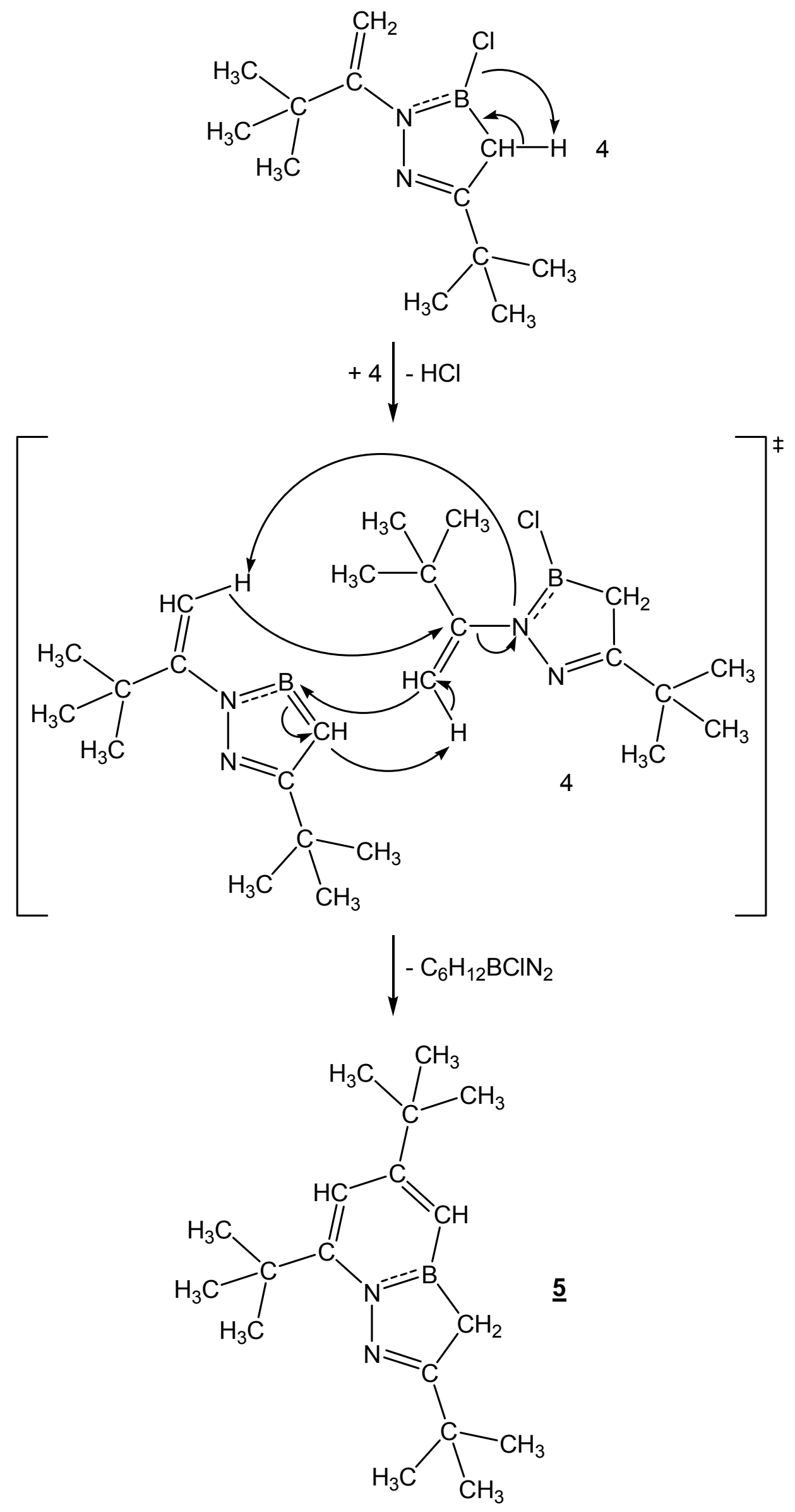

Schema 14: Möglicher Reaktionsweg für den Bicyclus $\underline{\mathbf{5}}$ 
Der Reaktionsmechanismus bedarf noch der Aufklärung. Allerdings konnte von $\underline{\mathbf{5}}$ eine Röntgenstrukturanalyse angefertigt werden:

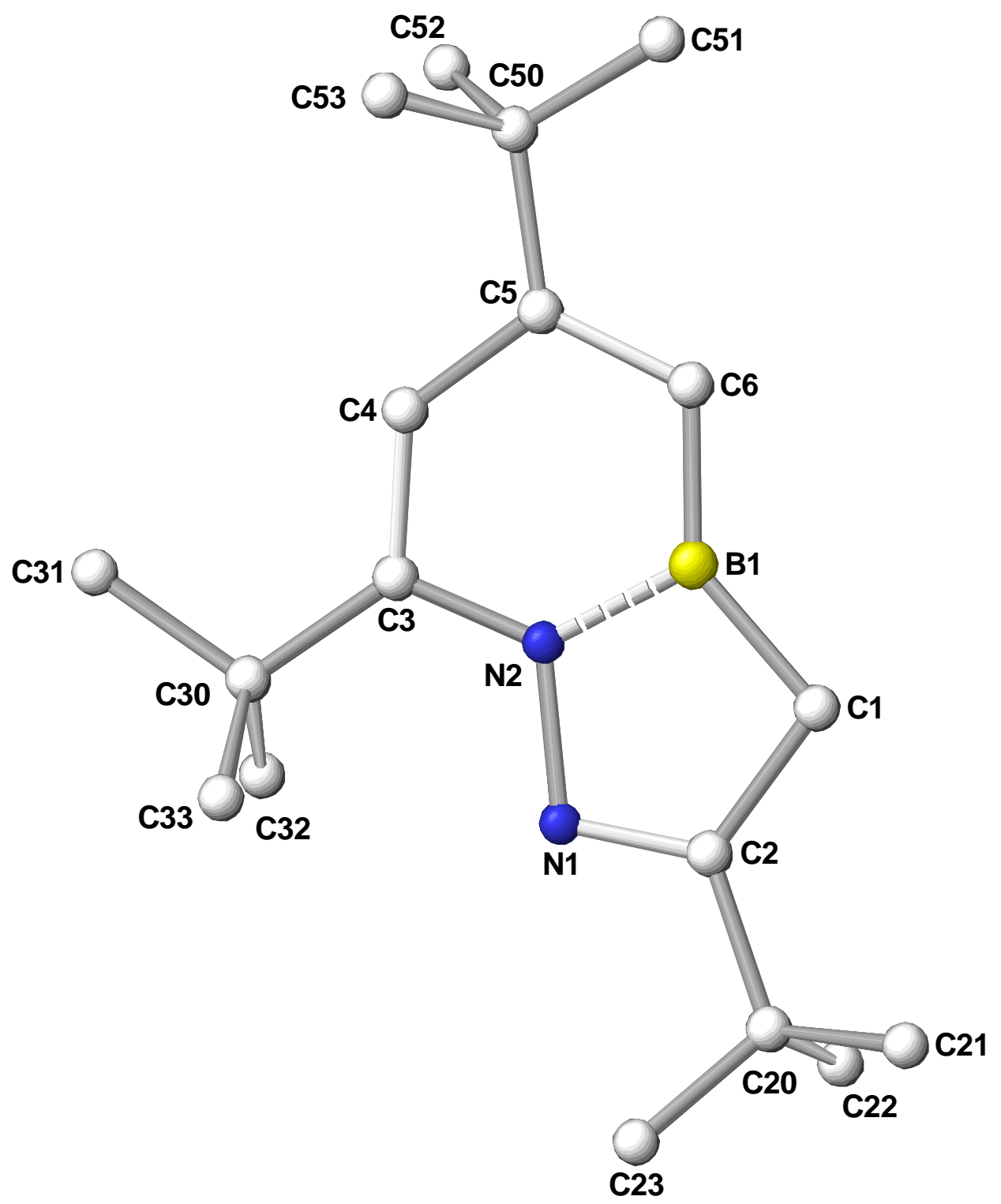

Abbildung 12: Struktur von Verbindung $\underline{\mathbf{5}}$ (ohne Wasserstoffatome)

\begin{tabular}{|c|c|c|c|}
\hline$N(1)-N(2)$ & 150,2 & $C(6)-B(1)$ & 149,1 \\
\hline$N(2)-B(1)$ & 142,3 & $\mathrm{~N}(1)-\mathrm{N}(2)-\mathrm{B}(1)$ & 111,8 \\
\hline$B(1)-C(1)$ & 157,5 & $N(2)-B(1)-C(1)$ & 104,0 \\
\hline$C(1)-C(2)$ & 150,7 & $B(1)-C(1)-C(2)$ & 102,1 \\
\hline $\mathrm{C}(2)-\mathrm{N}(1)$ & 128,5 & $C(1)-C(2)-N(1)$ & 115,5 \\
\hline $\mathrm{C}(3)-\mathrm{N}(2)$ & 135,8 & $\mathrm{C}(2)-\mathrm{N}(1)-\mathrm{N}(2)$ & 106,6 \\
\hline$C(4)-C(3)$ & 139,1 & $\Sigma$ Innenwinkel & 540,0 \\
\hline
\end{tabular}

Tabelle 2: Ausgewählte Bindungslängen $(\mathrm{pm})$ und -winkel $\left({ }^{\circ}\right)$ von Verbindung $\underline{\mathbf{5}}$ 
Der fünfgliedrige Diazaboracyclopenten-Ring ist mit einer Innenwinkelsumme von $540,0^{\circ}$ planar. Über $N(2)$ und $B(1)$ ist der Ring mit einem zweiten sechsgliedrigen Ring zu einem Bicyclus verknüpft. Die B(1)-N(2)-Bindung ist im Vergleich zu der bisher gezeigten Struktur von $\mathbf{X}$ um rund 18 pm auf 142,3 pm verkürzt, was für eine $\mathrm{B}=\mathrm{N}-$ Doppelbindung spricht (vgl. PAETZOLD ${ }^{16}$ ). Dafür ist die benachbarte NN-Bindung mit 150,2 pm um rund 10 pm länger als in $\mathbf{X}$.

Durch die Ausbildung der Aminoboran-Einheit sind die Doppelbindungen des an den fünfgliedrigen Ring gekoppelten sechsgliedrigen Ring konjugiert. Die Innenwinkelsumme dieses Rings beträgt $719,9^{\circ}$. Die minimale Abweichung von $0,1^{\circ}$ zum geometrischen Sechseck bestätigt die Planarität des Rings und somit die Ausbildung eines aromatischen $6 \pi-S y s t e m s$. Dies zeigt sich an den Bindungslängen der Kohlenstoffatome $C(3)-C(4)-C(5)-C(6)$. Alle drei Abstände liegen mit rund 140 pm im Bereich der CC-Bindungen von Phenylgruppen (vgl. auch Verbindung $\underline{7}$ in Kapitel 3.1.2.5). Die nachfolgende Abbildung verdeutlicht die Planarität des Doppelrings:

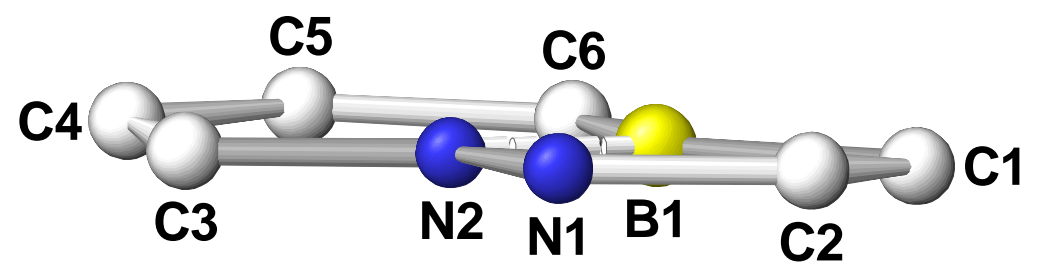

Abbildung 13: Seitenansicht der Ringebene des fünfgliedrigen Rings von Verbindung $\underline{\mathbf{5}}$ (ohne Alkylsubstituenten an $\mathrm{C}(2), \mathrm{C}(3)$ und $\mathrm{C}(5)$ )

Die $\mathrm{C}(3)-\mathrm{N}(2)$-Bindung ist mit $135,8 \mathrm{pm}$ länger als die Doppelbindung $N(1)=C(2)$ (128,5 pm), aber kürzer als eine C-N-Einfachbindung (140 - 142 pm). Das gleiche gilt für die $\mathrm{B}(1)-\mathrm{C}(6)$-Bindung, die im Vergleich $\mathrm{zu}$ der $\mathrm{B}(1)-\mathrm{C}(1)$-Einfachbindung (157,5 pm) mit 149,1 pm deutlich kürzer ist und somit einen partiellen B=C-Doppelbindungscharakter aufweist. Dies beweist noch einmal den aromatischen Charakter des Ringsystems. 


\subsubsection{Darstellung des 5-tert.-Butyl-2-(3,3-dimethylbutyliden-2)-3-chlor-3- phenyl-1,2-azaazonia-3-boratacyclopent-5-ens $\underline{6}$}

Mit Verbindung $\underline{\mathbf{3}}$ konnte durch Substitution eines Halogens an dem Azaazoniacyclopenten $\mathbf{X}$ ein $B$-Alkyl-B-Halogen-Azaazoniaboratacyclopenten dargestellt werden. Durch Umsetzung des monolithiierten Bis(tert.-butyl-methyl)ketazins mit einem Äquivalent Dichlorphenylboran in $n$-Hexan entsteht die analoge Arylverbindung $\underline{\mathbf{6}}$ in einem Schritt:

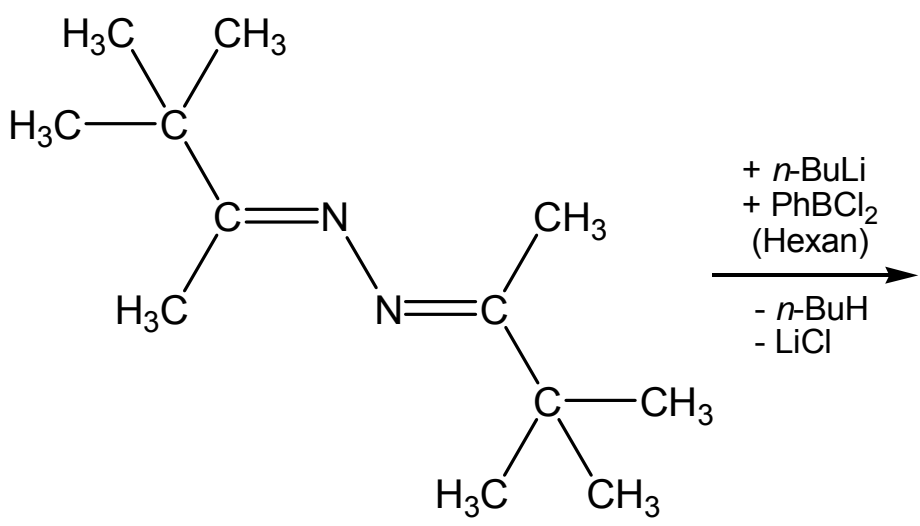

VIII<smiles></smiles>

$\underline{6}$

Schema 15: Darstellung des Azaazoniaboratacyclopentens $\underline{\mathbf{6}}$

Verbindung $\underline{\mathbf{6}}$ fällt als hellgelber Feststoff an.

\subsubsection{Substitution von 5-tert.-Butyl-2-(3,3-dimethylbutyliden-2)-3-chlor-3- phenyl-1,2-azaazonia-3-boratacyclopent-5-en}

Bei Zugabe von Lithiumorganylen zu organischen Verbindungen wird im allgemeinen bei höheren Temperaturen (Raumtemperatur und höher) die Substitution gegenüber der Metallierung bevorzugt. Das $B$-Chlor- $B$-Phenyl-Azaazoniaboratacyclopenten bildet dabei keine Ausnahme. Bei der Umsetzung von Verbindung $\underline{\mathbf{6}}$ mit sterisch anspruchsvollem tert.-Butyllithium entsteht bei Raumtemperatur in $n$-Hexan erwartungsgemäß Verbindung $\underline{7}$ : 


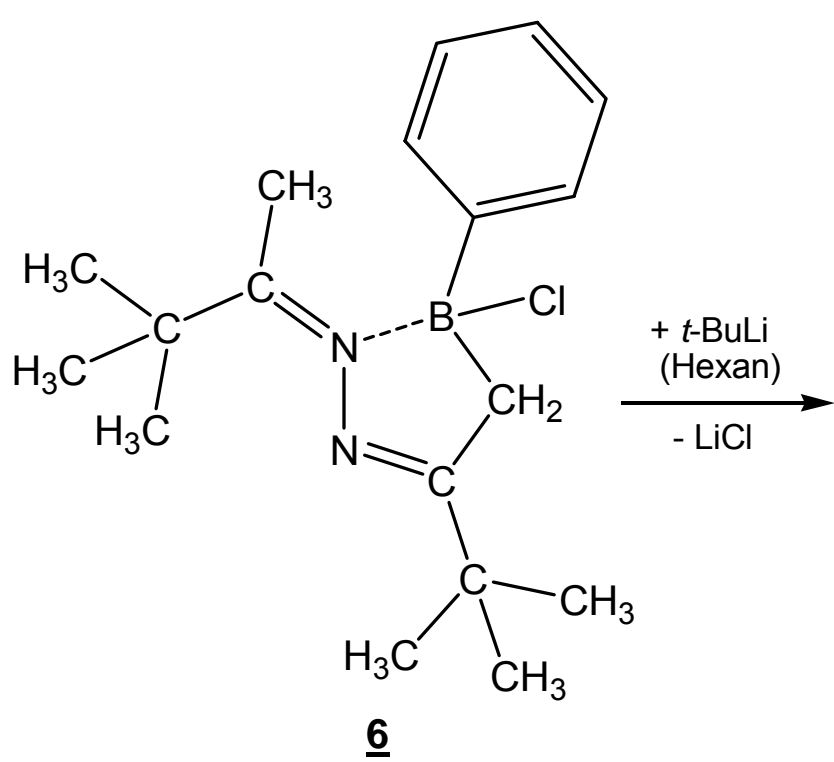<smiles></smiles>

Schema 16: Darstellung des Azaazoniaboratacyclopentens $\underline{7}$

Die Reaktion verläuft unvollständig. Man erhält etwa die Hälfte der Ausgangssubstanz bei der Aufarbeitung wieder zurück. Nebenprodukte werden nicht isoliert; NMR-Daten lassen auf ein polymeres Verbindungsgemisch schließen.

Verbindung $\underline{7}$ kristallisiert monoklin aus Hexan mit der Raumgruppe P2(1)/c: 


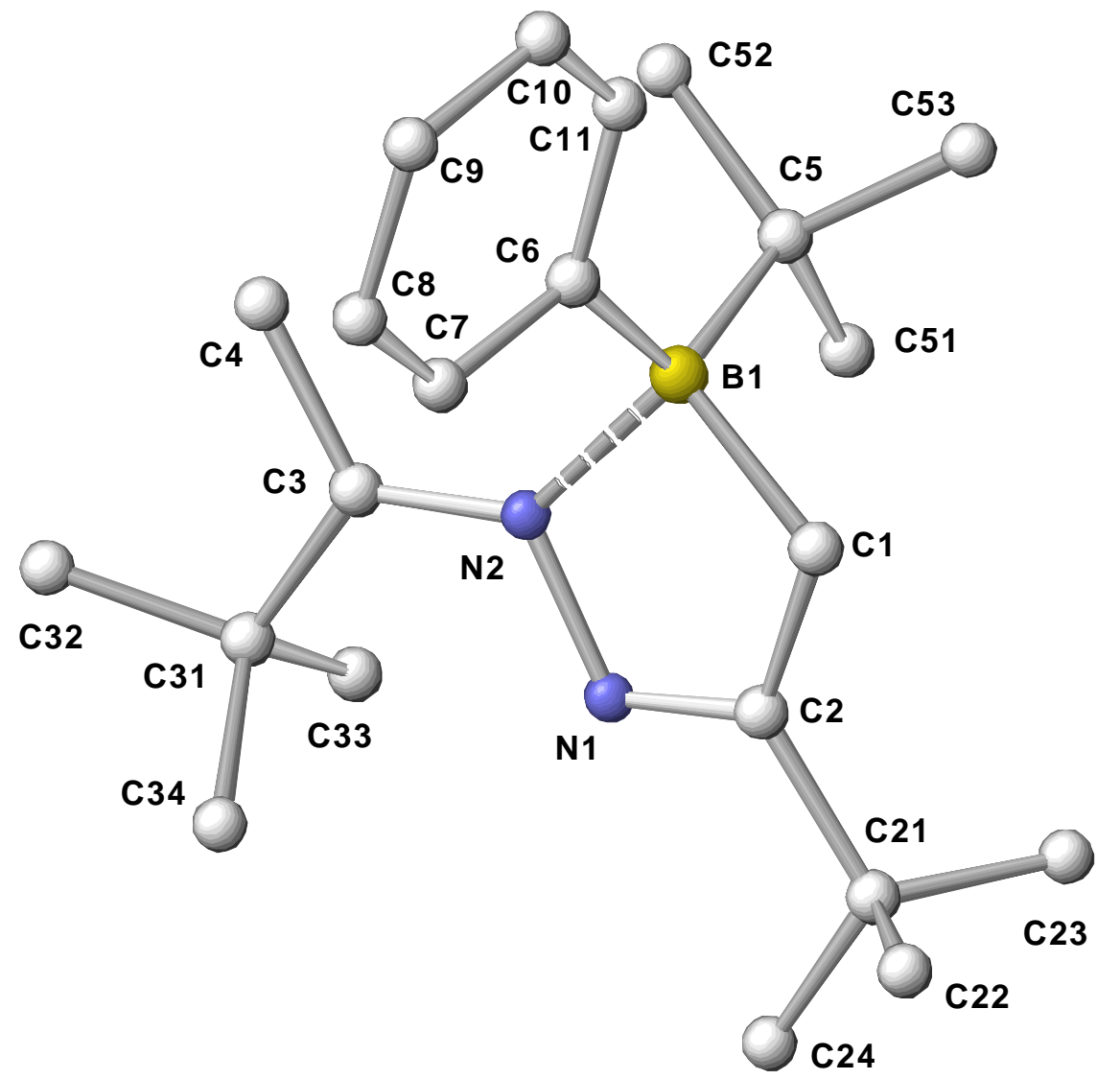

Abbildung 14: Struktur von Verbindung $\mathbf{Z}$ (ohne Wasserstoffatome)

\begin{tabular}{|c|c|c|c|}
\hline$N(1)-N(2)$ & 143,1 & $\mathrm{~N}(1)-\mathrm{N}(2)-\mathrm{B}(1)$ & 110,4 \\
\hline $\mathrm{N}(2)-\mathrm{B}(1)$ & 167,3 & $N(2)-B(1)-C(1)$ & 94,8 \\
\hline $\mathrm{B}(1)-\mathrm{C}(1)$ & 163,7 & $B(1)-C(1)-C(2)$ & 104,9 \\
\hline$C(1)-C(2)$ & 148,2 & $C(1)-C(2)-N(1)$ & 117,7 \\
\hline$C(2)-N(1)$ & 128,2 & $C(2)-N(1)-N(2)$ & 109,0 \\
\hline$C(3)-N(2)$ & 129,5 & $\Sigma$ Innenwinkel & 536,8 \\
\hline
\end{tabular}

Tabelle 3: Ausgewählte Bindungslängen $(\mathrm{pm})$ und -winkel $\left({ }^{\circ}\right)$ von Verbindung $\mathbf{z}$

Im Gegensatz zu Verbindung $\mathbf{X}$ ist der fünfgliedrige Ring nicht streng planar. Das Boratom steht aus der durch die $\mathrm{N}(2)-\mathrm{N}(1)-\mathrm{C}(2)-\mathrm{C}(1)$-Atome aufgespannten Ebene um $33 \mathrm{pm}$ heraus. Dies verdeutlicht auch die Innenwinkelsumme des Ringes, die mit $536,8^{\circ}$ um mehr als $3^{\circ}$ von einem geometrischen Vieleck abweicht. Dabei wird das Boratom von der sterisch sehr anspruchsvollen tert.-Butylgruppe aus der Ringebene herausgezogen: 


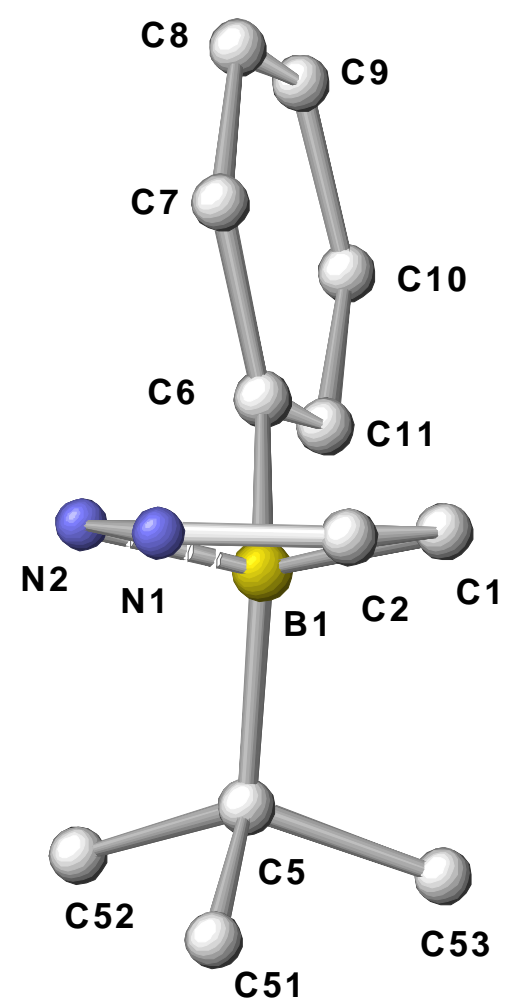

Abbildung 15: Seitenansicht der Ringebene von Verbindung $\mathbf{7}$ (ohne Alkylsubstituenten an N(2) und C(2))

Die anderen Bindungslängen und -winkel entsprechen weitgehend denen von Verbindung $X$ und auch den erwarteten Werten für eine solche Struktur ( $C=N$ 128,2 bzw. 129,5 pm, NN 143,1, CC 148,2 bis 154,1 pm). Abweichungen von $\underline{\mathbf{Z}}$ gegenüber $\mathbf{X}$ sind neben der nicht mehr vorhandenen Ringplanarität die Bindungsverlängerungen von N(2)-B(1) und B(1)-C(1) von 160,9 bzw. 159,4 pm auf 167,3 bzw. 163,7 pm. Dies lässt sich mit den Substituenten am Boratom begründen. Das Boratom wird durch die Alkyl- bzw. Arylsubstitution elektronenreicher. Dadurch wird vor allem die Boramminbindung, aber auch die Bormethylenbindung, geschwächt und verlängert. Den Einfluss der unterschiedlichen Borsubstitution zeigt auch der Ringinnenwinkel an $B(1)$. Dieser wird von $99,7^{\circ}$ auf $94,8^{\circ}$ deutlich verkleinert. Damit wird er den größeren sterischen Ansprüchen des tert.-Butyl- bzw. des Phenylrestes gegenüber den beiden Chloratomen an $\mathbf{X}$ gerecht. 


\subsubsection{Dehydrohalogenierung von 5-tert.-Butyl-2-(3,3-dimethylbutyliden-2)-3- chlor-3-phenyl-1,2-azaazonia-3-boratacyclopent-5-en}

Eine Umsetzung der Verbindung $\underline{\mathbf{6}}$ mit 2 Äquivalenten Triethylamin führt zu dem aus dem Arbeitskreis MELLER bekannten B-Phenyl-Diazaboracyclopenten XII ${ }^{7}$ :

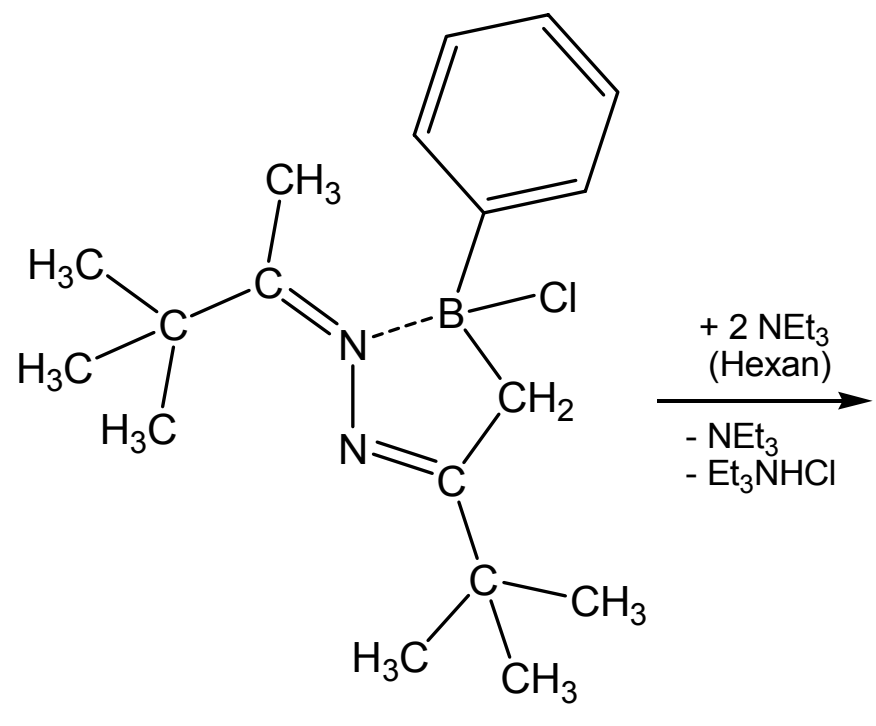

$\underline{6}$<smiles>C=C(N1N=C(C(C)(C)C)CB1c1ccccc1)C(C)(C)C</smiles>

XII

Schema 17: Dehydrohalogenierung der Verbindung $\underline{6}$

Bei der Reaktion entsteht ein Produktgemisch. Die Dehydrohalogenierung ist über den Zusatz von Triethylamin nicht quantitativ zu erreichen. Eine vollständige Umsetzung ist mit $n$-Butyllithium bei tiefen Temperaturen $\left(-60^{\circ} \mathrm{C}\right)$ in $n$-Hexan weitgehend möglich. Ähnliche Ergebnisse sind aus der Bor-Hydrazon-Chemie bekannt. Dort konnten aus acyclischen $\mathrm{N}$-Hydrazonyl-Aminoboranen in einem zweiten Metallierungsschritt fünfgliedrige Ringe erhalten werden ${ }^{21,22,23}$. Eine Deprotonierung über starke Alkyllithiumbasen mit anschließender Lithiumhalogenid-Eliminierung wird häufig zu einer inter- wie intramolekularen Bindungsbildung genutzt. Bei tiefen Temperaturen ist dieser Reaktionsweg gegenüber der Substitution bevorzugt. 


\subsubsection{Darstellung des 3-Bis(trimethylsilyl)amino-5-tert.-butyl-2-(3,3dimethyl- butyliden-2)-1,2-diaza-3-boracyclopent-5-ens $\underline{9}$}

Im Arbeitskreis MELLER wurden bislang acyclische Aminoboryl-Ketazine und zu Verbindung $\underline{\mathbf{9}}$ analoge Diazaboracyclopentene isoliert ${ }^{7,23}$. Azaazoniaboratacyclopentene mit aminosubstituierten Bor sind nicht bekannt. Die Substitution an einem halogenfunktionellen Ring gelang mit Lithiumaminoverbindungen bisher nicht. Erfolgreiche Darstellungen verliefen immer über die Umsetzung eines Aminoborans mit einem lithiierten Ketazin.

Ein Versuch der Halogensubstitution an X mit Lithium-(bis(trimethylsilyl)amid) führt zu keinem befriedigenden Ergebnis. Die Umsetzung eines Aminodifluorborans mit monolithiiertem Ketazin VIII führt dagegen zu dem Produkt $\underline{\mathbf{9}}$ :

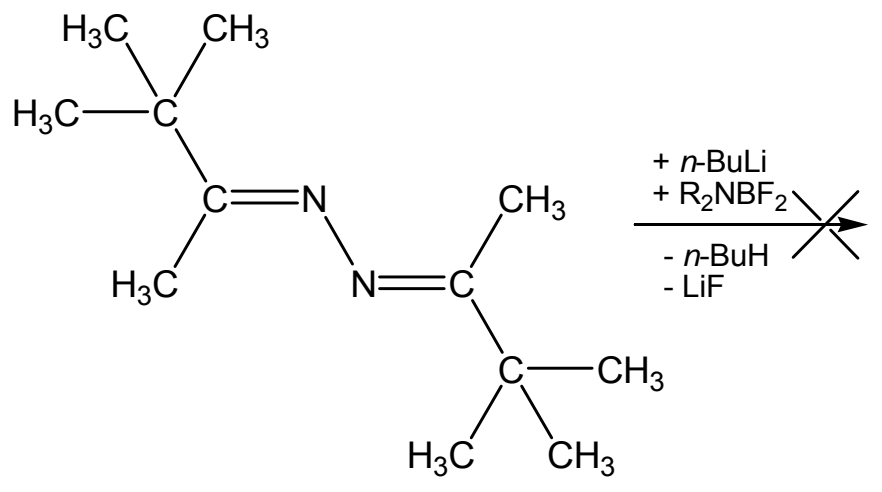<smiles></smiles>

VIII

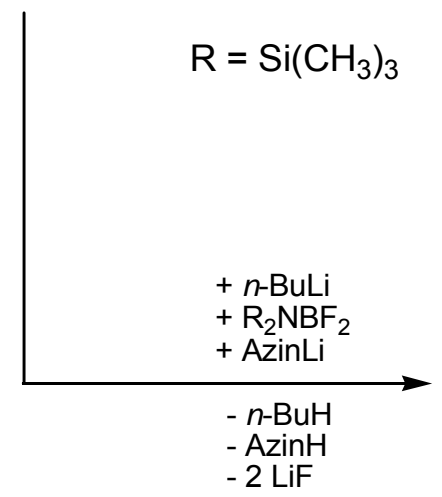<smiles>C=C(N1N=C(C(C)(C)C)CB1N([Si](C)(C)C)[Si](C)(C)C)C(C)(C)C</smiles>

Schema 18: Umsetzung des monolithiierten VIII mit einem Aminodihalogenboran 
Dabei entsteht $\underline{\mathbf{9}}$ durch Abspaltung zweier Moleküle Lithiumfluorid. Da monosubstituierte Ketazine bekannt sind, muss - zumindest intermediär - ein monolithiiertes Ketazin existieren und somit eine intermolekulare Umlithiierung erfolgen. Dies ist von Hydrazonen bekannt ${ }^{21,56}$ (siehe auch GI. 3). Verbindung 8 konnte genau so wenig isoliert werden, wie eine isomere Kette. Die ${ }^{11} \mathrm{~B}-\mathrm{NMR}$-Daten weisen auf das Vorhandensein mehrerer borhaltiger Produkte hin; eine Zuordnung zu definierten Verbindungen ist nicht möglich, da bei der thermischen Aufreinigung Polymerisationsprozesse die Isolierung der einzelnen Komponenten verhindern. Ein acyclisches Aminoboryl-Ketazin wurde im Arbeitskreis MELLER dargestellt und seine Struktur mit einer Röntgenanalyse belegt ${ }^{7}$.

Eine direkte Synthese von $\underline{\mathbf{9}}$ über die dilithiierte Spezies von VIII verläuft erwartungsgemäß mit besserer Ausbeute. Verbindung $\underline{\mathbf{9}}$ ist ein gelber Feststoff, der unzersetzt sublimierbar ist.

\subsubsection{Versuchte Darstellung eines B-Diamino-Azaazoniaboratacyclopentens}

Nach der erfolgreichen Darstellung des Alkyl-Aryl-substituierten Azaazoniaboratacyclopentens $\underline{7}$ liegt der Syntheseversuch eines doppelt aminosubstituierten Ringes nahe. Da die Darstellung eines $B$-Amino- $B$-Halogen-substituierten Azaazoniaboratacyclopentens nicht gelang, entfällt die Substitution eines Halogens als mögliche Syntheseroute.

Die versuchte Umsetzung des monolithiierten Ketazins VIII mit Diaminohalogenboranen brachte nicht das gewünschte Ergebnis. Trotz erfolgreicher Lithiierung (durch Auswiegen des entstandenen Butans überprüfbar) findet keine Reaktion statt: 
<smiles>C/C(=N\N=C(/C)C(C)(C)C)C(C)(C)C</smiles>

VIII<smiles></smiles>

10,11

\begin{tabular}{c|c|c} 
& 10 & 11 \\
\hline $\mathrm{R}$ & $\mathrm{iPr}$ & $\mathrm{SiMe}_{3}$ \\
\hline Hal & $\mathrm{Cl}$ & $\mathrm{F}$
\end{tabular}

Schema 19: Umsetzung des Ketazins VIII mit Diaminohalogenboranen

Sowohl Ketazin als auch das Diaminohalogenboran können destillativ aus dem Reaktionsgemisch zurückgewonnen werden (als Nebenprodukt entsteht eine schwarze ölige Flüssigkeit undefinierter Zusammensetzung).

Vermutlich unterbleibt eine intramolekulare Cyclisierung über eine $\mathrm{N} \rightarrow \mathrm{B}$-Adduktbildung, da diese ein ausreichend Lewis-acides Borzentrum voraussetzt. Durch die beiden Aminosubstituenten ist das Boratom vergleichbar elektronenreich und eine intramolekulare Absättigung ist somit nicht erforderlich. Im Arbeitskreis MELLER sorgte in einem Fall eine Aminogruppe an der Borverbindung für ein Unterbleiben der intramolekularen Cyclisierung ${ }^{7,23}$. 


\subsubsection{Kristallstruktur des 2,6-tert.-Butoxi-4,8-diisopropyl-1,5-diaza- 2,6-dibora-bicyclo[3.3.0]octa-3,7-diens XV}

Die Erzeugung von röntgentauglichen Kristallen eines zweiten bicyclischen Systems gelang bei der erneuten Darstellung eines Octadiens aus Bis(isopropyl-(trimethylsilyl)methyl)ketazin XIII und anschließender Substitution der verbliebenen Halogene durch Butoxisubstituenten ${ }^{8}$ :

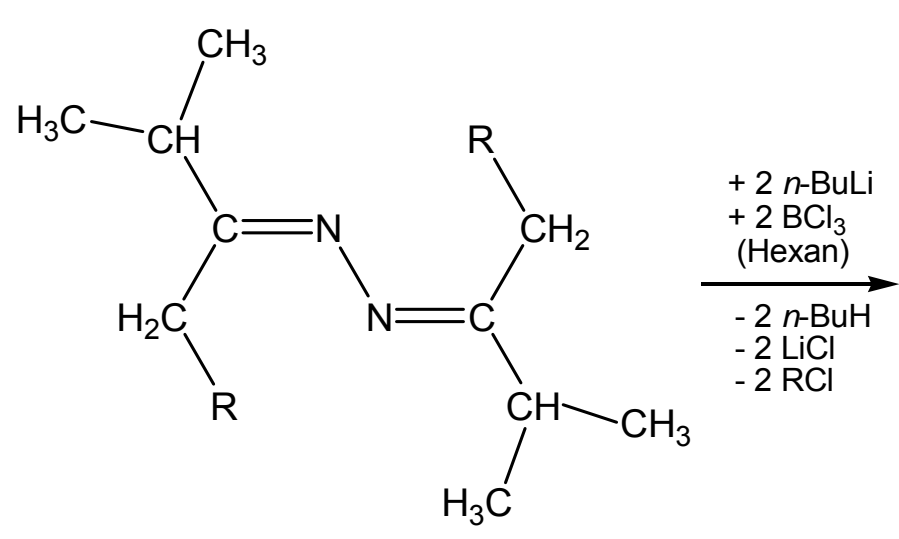

XIII $\mathrm{R}=\mathrm{Si}\left(\mathrm{CH}_{3}\right)_{3}$<smiles></smiles>

XIV<smiles>CC(C)(C)OC(C)(C)C(C)(C)C</smiles><smiles></smiles>

Schema 20: Darstellung des Bicyclus XV

Die Struktur von $\mathbf{X V}$ ist erwartungsgemäß nahezu identisch zu der im Arbeitskreis MELLER bereits zuvor dargestellten analogen Verbindung aus Bis(tert.-butyl-methyl)ketazin ${ }^{6}$. 


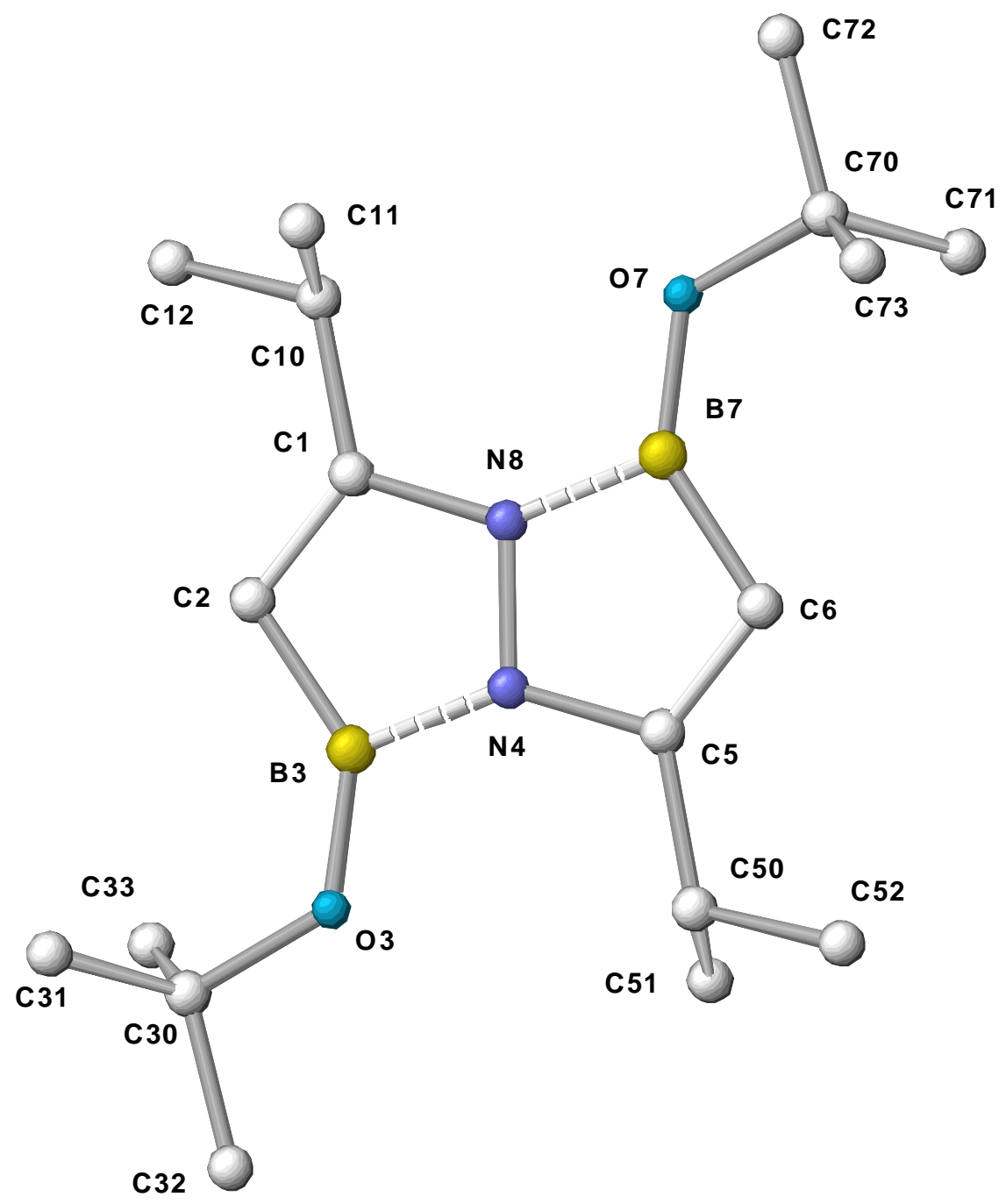

Abbildung 16: Struktur von Verbindung XV (ohne Wasserstoffatome)

$\begin{array}{|cc|}\mathrm{C}(1)-\mathrm{C}(2) & 135,6 \\
\mathrm{C}(2)-\mathrm{B}(3) & 153,1 \\
\mathrm{~B}(3)-\mathrm{N}(4) & 145,7 \\
\mathrm{~N}(4)-\mathrm{N}(8) & 140,0 \\
\mathrm{~N}(8)-\mathrm{C}(1) & 140,7 \\
\mathrm{~B}(3)-\mathrm{O}(3)-\mathrm{C}(30) & 125,3\end{array} \mid$\begin{tabular}{ccc|}
$\mathrm{C}(1)-\mathrm{C}(2)-\mathrm{B}(3)$ & 106,7 \\
$\mathrm{C}(2)-\mathrm{B}(3)-\mathrm{N}(4)$ & 103,6 \\
$\mathrm{~B}(3)-\mathrm{N}(4)-\mathrm{N}(8)$ & 109,6 \\
$\mathrm{~N}(4)-\mathrm{N}(8)-\mathrm{C}(1)$ & 107,7 \\
$\mathrm{~N}(8)-\mathrm{C}(1)-\mathrm{C}(2)$ & 112,3 \\
& & \\
\hline
\end{tabular}

Tabelle 4: Ausgewählte Bindungslängen $(\mathrm{pm})$ und -winkel $\left({ }^{\circ}\right)$ von Verbindung XV

Die Summen der Innenwinkel der beiden fünfgliedrigen Rings bestätigen mit rund $540^{\circ}\left(\mathrm{C}(1)-\mathrm{C}(2)-\mathrm{B}(3)-\mathrm{N}(4)-\mathrm{N}(8)=539,9^{\circ}, \mathrm{C}(5)-\mathrm{C}(6)-\mathrm{B}(7)-\mathrm{N}(8)-\mathrm{N}(4)=540,1^{\circ}\right)$ die Planarität jedes Einzelrings, während die Winkelsummen an $\mathrm{N}(4)$ und $\mathrm{N}(8)$ mit je $360,0^{\circ}$ die $\mathrm{sp}^{2}$-Hybridisierung der Stickstoffatome verdeutlichen. Somit sind zwei planare 
Ringe aneinander zu einem planaren bicyclischen achtgliedrigen Ringsystem gekoppelt.

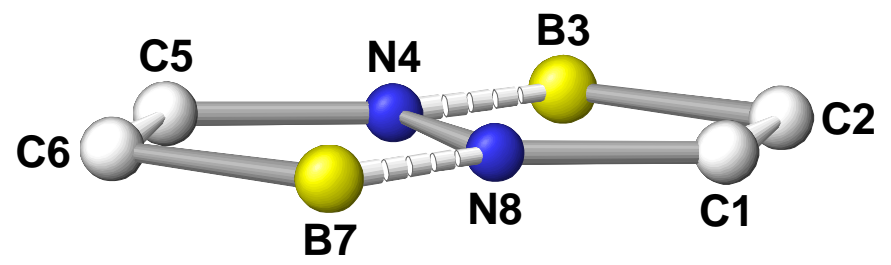

Abbildung 17: Seitenansicht der Ringebene von Verbindung XV (ohne Substituenten an $\mathrm{C}(1), \mathrm{B}(3), \mathrm{C}(5)$ und $\mathrm{B}(7)$ )

Der Winkel an $\mathrm{O}(3)$ wird mit $125,3^{\circ}$ den sterischen Ansprüchen der terminalen tert.Butylgruppe gerecht und ist im Vergleich zum Wassermolekül um rund $20^{\circ}$ geweitet. Auch die Bindungslängen entsprechen den schon weiter oben vorgestellten Ringsystemen. Die Abstände von $\mathrm{C}(1)-\mathrm{C}(2), \mathrm{C}(5)-\mathrm{C}(6), \mathrm{B}(3)-\mathrm{N}(4)$ und $\mathrm{B}(7)-\mathrm{N}(8)$ weisen deutlich auf Doppelbindungen hin $(C=C \sim 135$ pm, $B=N \sim 145$ pm). Die anderen Bindungen entsprechen typischen Einfachbindungslängen (z.B. N(4)-N(8) 140,0 pm, $\mathrm{N}(4)-\mathrm{C}(5) 138,8 \mathrm{pm})$. 


\subsection{Phosphor-Azin-Chemie}

\subsubsection{Synthese von fünfgliedrigen phosphorhaltigen Ringen}

Aus der Bor-Azin-Chemie gewonnene Erkenntnisse können auf Umsetzungen von Ketazinen mit Phosphanen übertragen werden. Dies soll die Darstellung neuer Ringe ermöglichen. Zunächst steht die Umsetzung von Ketazin mit Phosphanen im molaren Verhältnis 1:1 im Vordergrund.

\subsubsection{Versuchte Darstellung des zu Verbindung 4 analogen Diazaphospha- cyclopentadiens}

Die beste Vergleichsmöglichkeit zwischen bor- und phosphorhaltigen fünfgliedrigen Ringen würde die Darstellung des zu Verbindung 4 analogen Rings bieten:<smiles></smiles>

Abbildung 18: Vergleich der Borverbindungen $\mathbf{X}$ und 4 mit der phosphorhaltigen Verbindung 12

Eine Darstellung erfordert auch in diesem Fall eine doppelte Dehydrohalogenierungsreaktion. Eine zu Verbindung $\mathbf{X}$ analoge Phosphorverbindung ist nicht isolierbar. Die Struktur von $\mathbf{X}$ setzt einen intramolekularen Ringschluss durch eine LewisSäure-Base-Reaktion voraus. Die Darstellung eines acyclisches Systems wäre aber denkbar.

Die Reaktion von Bis(tert.-butyl-methyl)ketazin mit einem Moläquivalent Trichlorphosphan in Anlehnung an SCHMIDPETER ${ }^{24,25,26,27}$ führt zu folgendem Ergebnis: 


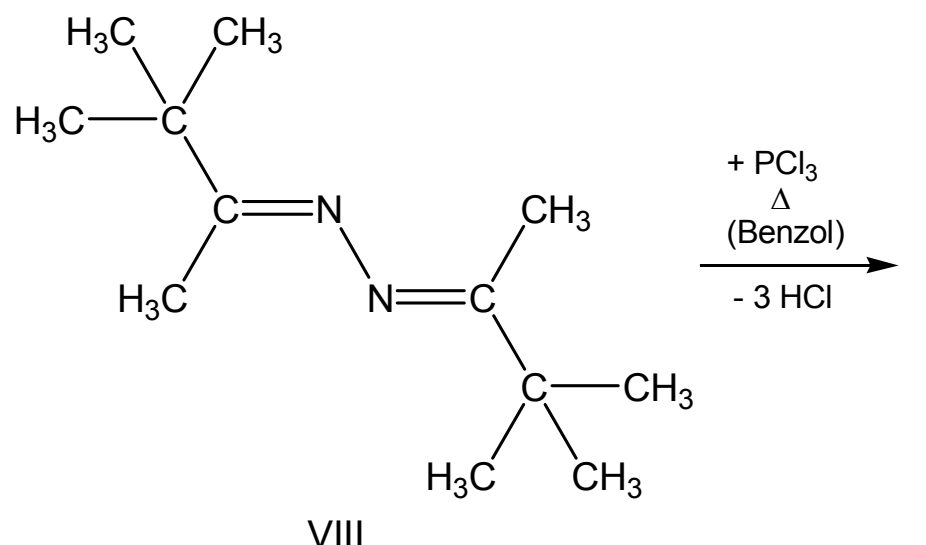<smiles></smiles>

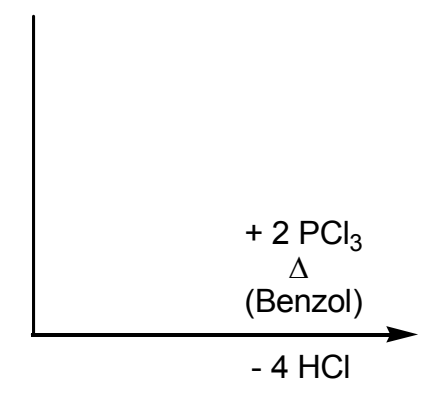<smiles>CC(C)(C)C1=CP(Cl)N2C(C(C)(C)C)=CP(Cl)N12</smiles>

Schema 21: Reaktion von Verbindung VIII mit $\mathrm{PCl}_{3}$

Die zuvor beschriebene Verbindung 12 ist nicht isolierbar. Es entsteht ein Gemisch aus etwa 60 \% 5-tert.-Butyl-2-(3,3-dimethylbut-1-en-2-yl)-1,2-diaza-3-phosphacyclopenta-3,5-dien $\underline{13}$ und $20 \%$ 4,8-Di-tert.-butyl-2,6-dichlor-1,5-diaza-2,6-diphosphabicyclo[3.3.0]octa-3,7-dien 14. Beide Verbindungen sind hellgelbe bis gelbe Feststoffe, die sich durch fraktionierte Sublimation voneinander trennen lassen. Dabei liegt Verbindung $\underline{13}$ als ein gegen Hydrolyse und Oxidation stabiles Pulver vor. Selbst gezielte Reaktionen mit Wasser, Kaliumhydroxid oder Schwefel führen zu keinen Additions- oder Oxidationsprodukten. Darin gleicht dieser Ring den in der Literatur bekannten Phospholen ${ }^{24,25}$. Die Stabilität gründet sich auf die Ausbildung eines aromatischen 6ா-Systems mit Beteiligung zweier konjugierter Doppelbindungen $(\mathrm{N}=\mathrm{C}$ und $\mathrm{P}=\mathrm{C})$ und eines freien Elektronenpaares des Stickstoffatoms (PN). Zudem ist die exocyclische Butyliden-Doppelbindung ebenfalls konjugiert.

Der Bicyclus $\underline{14}$ wird im nachfolgenden Kapitel 3.2.3.1 näher beschrieben. 


\subsubsection{Darstellung des 5-tert.-Butyl-2-(3,3-dimethylbut-1-en-2-yl)-3-chlor-3,4- dihydro-1,2-diaza-3-phosphacyclopenta-3,5-diens $\underline{13}$ über Lithium- derivate des Bis(tert.-butyl-methyl)ketazins}

Da eine Synthese von Verbindung 12 über die in der Phospholdarstellung üblichen Methoden nicht gelang, wurde Trichlorphosphan mit dem dilithiierten Ketazin VIII umgesetzt:

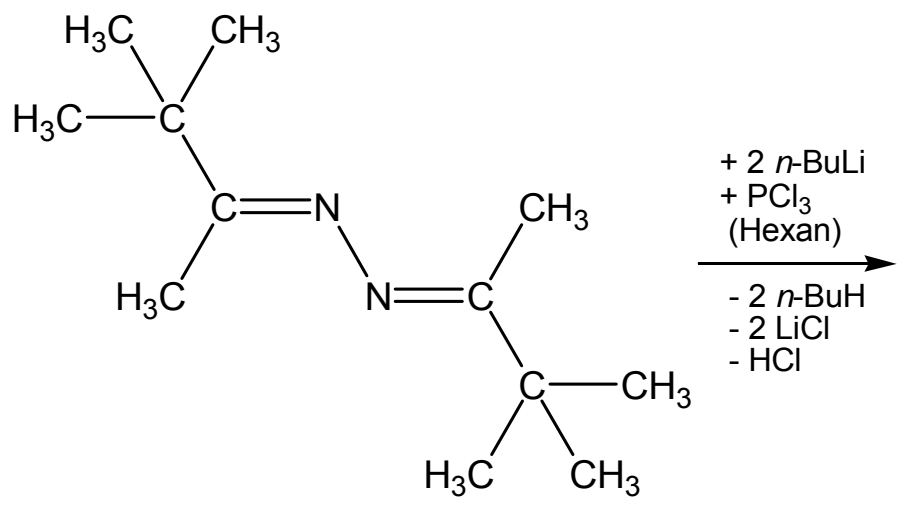<smiles>C=C(n1nc(C(C)(C)C)cp1)C(C)(C)C</smiles>

VIII

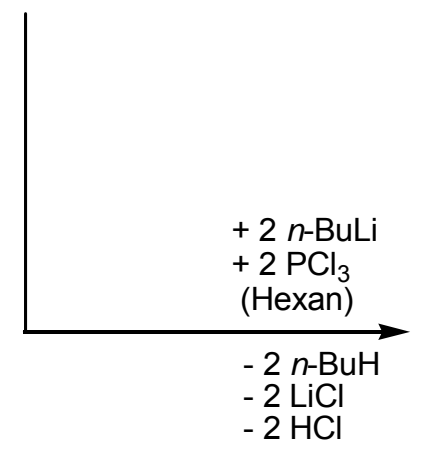<smiles>CC(C)(C)C1=CP(Cl)N2C(C(C)(C)C)=CP(Cl)N12</smiles>

Schema 22: Reaktion des dilithiierten Ketazins VIII mit $\mathrm{PCl}_{3}$

Wiederum konnten ausschließlich die Verbindungen $\underline{13}$ und $\underline{14}$ isoliert werden. Als Nebenprodukt entsteht ein zu Nadeln kristallisierender, farbloser Feststoff, der durch NMR-Messungen als das Hydrochlorid des Ketazins VIII identifiziert wurde. Eine Röntgenstrukturanalyse des Bis(tert.-butyl-methyl)ketazinhydrochlorids $\underline{\mathbf{1 5}}$ konnte angefertigt werden (vgl. Abb. 19). 


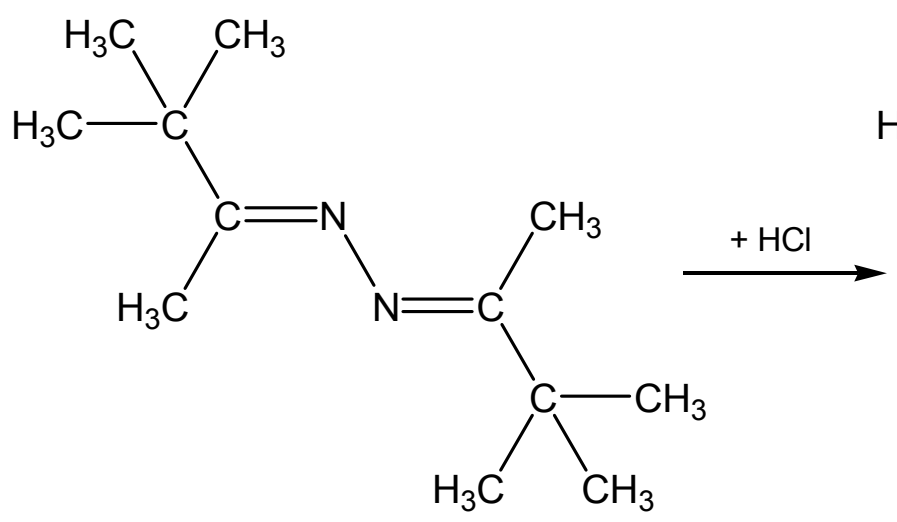

VIII

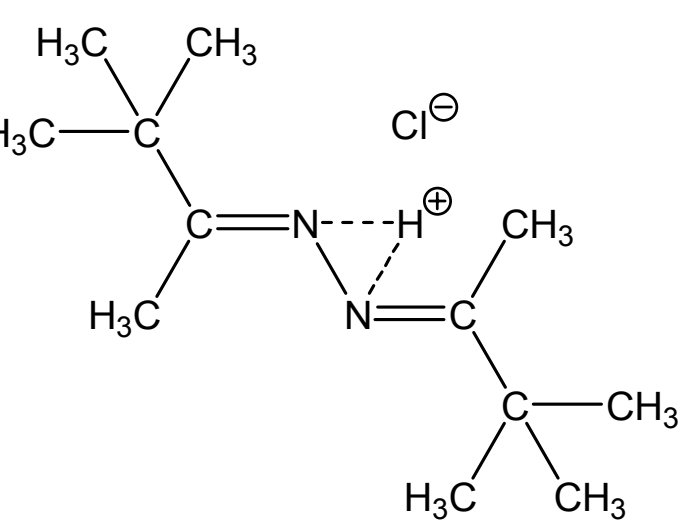

15

Schema 23: Entstehung des Hydrochlorids $\underline{\underline{15}}$

Chlorwasserstoffmoleküle, die in der Reaktion von Ketazin VIII mit Trichlorphosphan freigesetzt werden (vgl. Schema 21 und 22), fällen noch nicht umgesetztes Azin als Salz aus dem unpolaren Lösungsmittel. Die Bildung von Verbindung $\underline{\mathbf{1 5}}$ in der Reaktion nach Schema 21 wird durch das thermische Austreiben des Chlorwasserstoffgases aus dem Lösungsmittel unterbunden. Bei der direkten Umsetzung von Ketazin VIII mit trockenem HCl-Gas ohne Lösungsmittel entsteht $\underline{15}$ quantitativ in einer exothermen Reaktion.

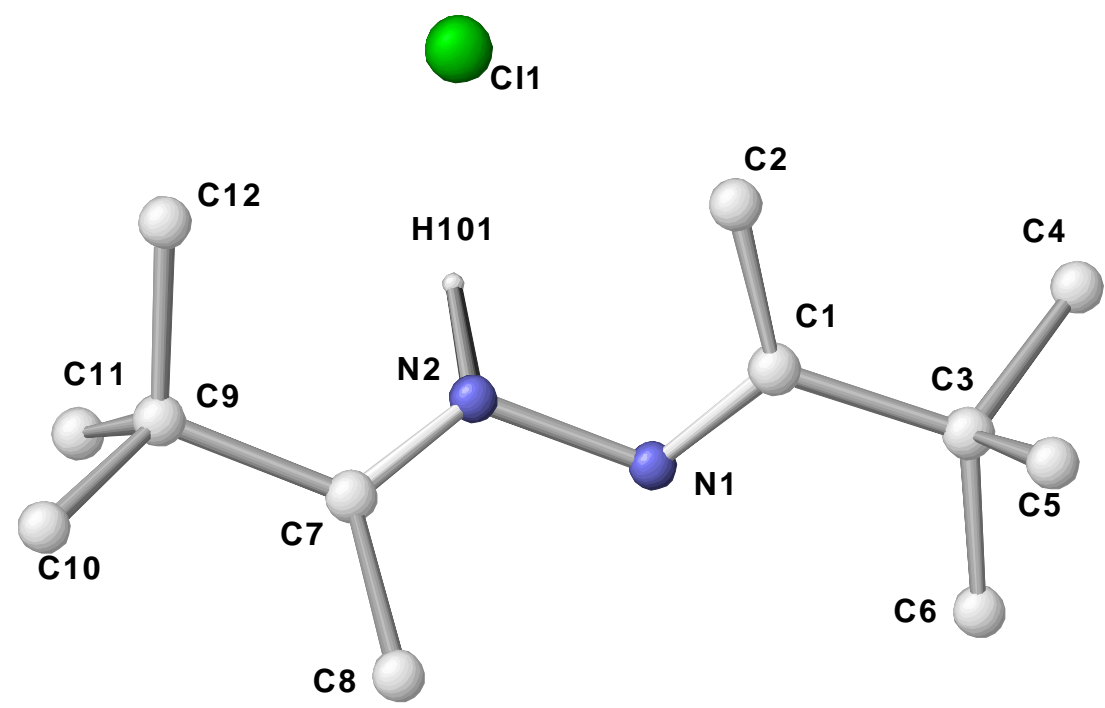

Abbildung 19: Struktur von Verbindung $\underline{\mathbf{1 5}}$ (ohne kohlenstoffgebundene Wasserstoffatome)

Deutlich ist das um $122^{\circ}$ tordierte Ketazingrundgerüst zu erkennen. Im Festkörper ist ein Wasserstoffatom an eines der beiden Ketazin-Stickstoffatome gebunden. Im ${ }^{1} \mathrm{H}-\mathrm{NMR}-\mathrm{Spektrum}$ findet sich lediglich ein breites $\mathrm{NH}$-Signal im stark sauren Bereich 
bei 15,5 ppm. Da die beiden ternären $\mathrm{C}=\mathrm{N}-$ Kohlenstoffatome im ${ }^{13} \mathrm{C}-\mathrm{NMR}-S p e k t r u m$ nur ein Signal liefern, liegt entweder ein symmetrischer Molekülaufbau und somit ein delokalisiertes Proton in Lösung vor, oder das Proton ist zwar an ein Stickstoffatom gebunden, aber die Struktur fluktuiert so rasch, dass die beiden Signale auf der NMR-Zeitskala nicht getrennt sichtbar sind.

\subsubsection{Gleichgewichtsreaktionen des Diazaphosphacyclopentadiens $\underline{13}$ mit Chlorwasserstoff}

Die Reaktion von Ketazin VIII mit Trichlorphosphan ist eine Gleichgewichtsreaktion, bei der durch kontinuierliche Abtrennung des entstehenden Chlorwasserstoffs das Gleichgewicht auf die Seite der Produkte verschoben wird (vgl. Schema 21). Eine Umkehrung des Gleichgewichts durch Zugabe von Chlorwasserstoff zu Verbindung $\underline{13}$ lässt sich wie folgt darstellen:<smiles>C=C(n1nc(C(C)(C)C)cp1)C(C)(C)C</smiles><smiles>C=C(N1N=C(C(C)(C)C)CP1Cl)C(C)(C)C</smiles>

$\underline{13}$
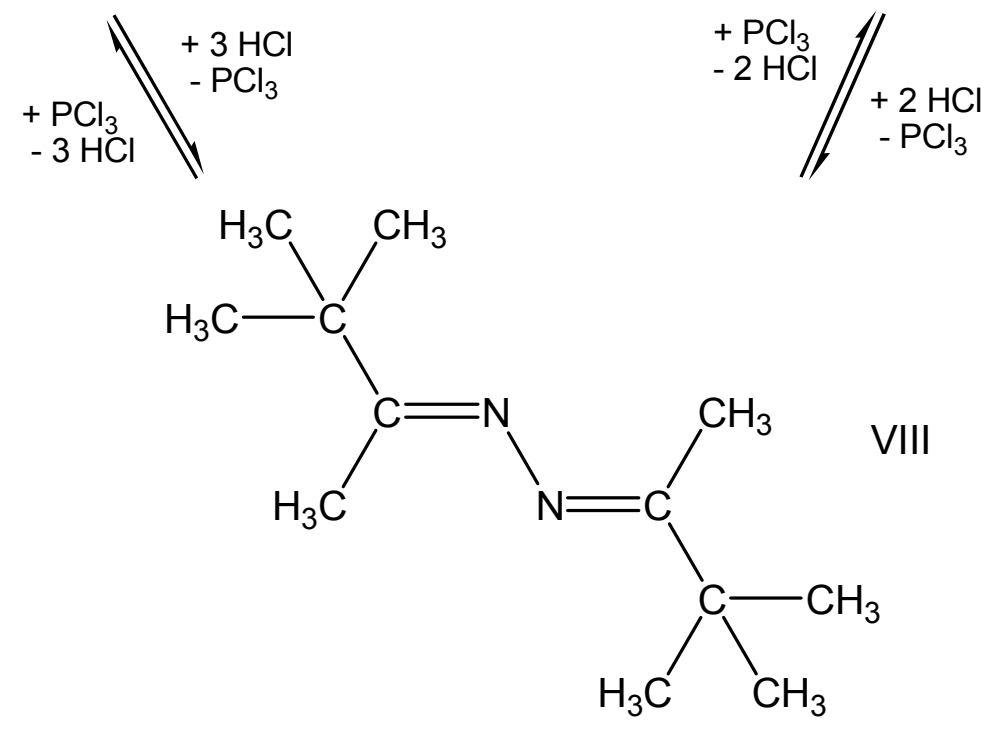

Schema 24: Gleichgewichtsreaktion des zwischen dem Phosphol $\underline{13}$ und dem Ketazin VIII 
Bei dieser Reaktion lässt sich Verbindung $12 \mathrm{im}$ Produktgemisch mit Hilfe von ${ }^{31} \mathrm{P}$ NMR-Spektren nachweisen. Die Isolierung ist bisher nicht gelungen. Eine thermische Aufarbeitung durch Destillation oder Sublimation führt in jedem Fall zu einer erneuten Dehydrochlorierung des 1,2-Diaza-3-phosphacyclopent-5-ens 12 und zur Bildung von Verbindung $\underline{13}$. Bei Zugabe eines Überschusses Chlorwasserstoff ist $\underline{13}$ wieder komplett in das Ketazin VIII und Trichlorphosphan überführbar. Diese lassen sich destillativ aus dem Produktgemisch zurückgewinnen.

Für die bicyclische Verbindung $\underline{14}$ lässt sich ein ähnliches Gleichgewicht formulieren. Dabei sind jedoch keine weiteren Zwischenstufen nachweisbar.

\subsubsection{Darstellung des 3,5-Di-tert.-butyl-2-(3,3-dimethylbut-1-en-2-yl)-1,2- diaza-3-phosphacyclopent-5-ens $\underline{16}$}

Da Verbindung 12 nicht isoliert wurde, ist eine Alkylsubstitution analog zur Bildung von Verbindung $\underline{3}$ nicht möglich. Die Umsetzung eines alkylsubstituierten Phosphans mit dem Bis(tert.-butyl-methyl)ketazin sollte aber ebenso das gewünschte Produkt liefern. Dabei stellte sich die thermische Dehydrochlorierung in der Siedehitze von Benzol oder Toluol als effektiver heraus, als die Lithiumchlorid-Eliminierung bei der Umsetzung mit dem dimetallierten Derivat des Ketazins. Das Produkt $\underline{16}$ konnte auf beiden Wegen erhalten werden.

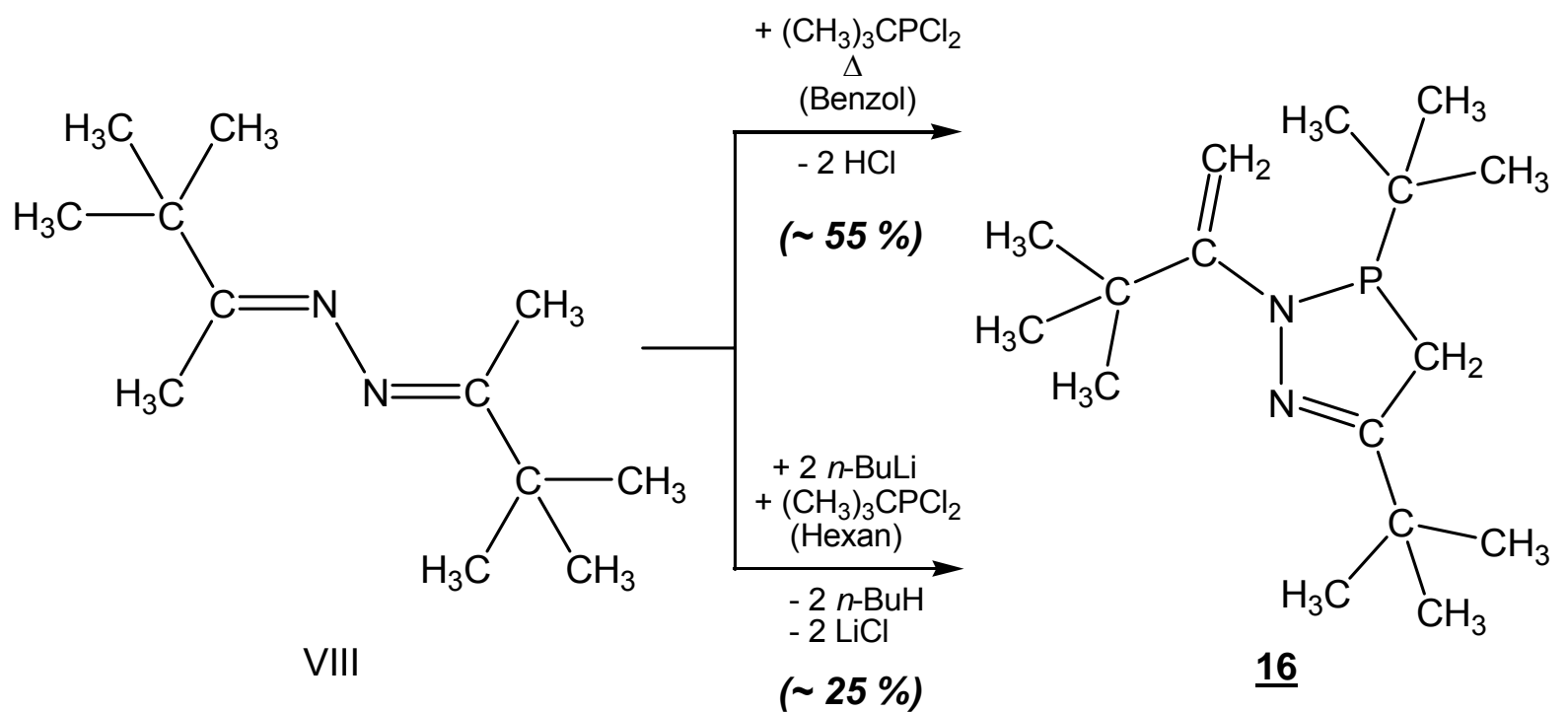

Schema 25: Darstellung des Dihydrophosphols $\underline{16}$ 
Verbindung $\underline{16}$ fällt dabei als gelber Feststoff an, der unter trockener Inertgasatmosphäre einige Wochen stabil ist. Im Gegensatz zu dem aromatischen Ring $\underline{13}$ ist $\underline{16}$ gegenüber Feuchtigkeit, Oxidationsmitteln und Basen sehr empfindlich.

\subsubsection{Oxidation des 3,5-Di-tert.-butyl-2-(3,3-dimethylbut-1-en-2-yl)-1,2-diaza-} 3-phosphacyclopent-5-ens $\underline{16}$

Bei dem Versuch, die durch NMR-spektroskopische und massenspektrometrische Daten charakterisierte Verbindung $\underline{\mathbf{1 6}}$ auch durch eine Röntgenstruktur zu belegen, konnte nur das Oxidationsprodukt aufgeklärt werden.

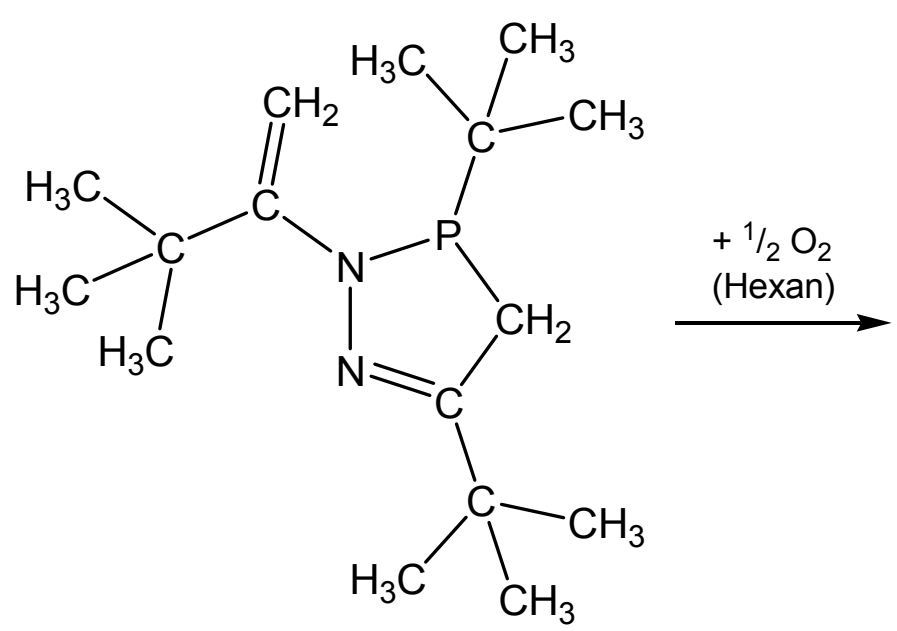

16<smiles>C=C(N1N=C(C(C)(C)C)CP1(=O)C(C)(C)C)C(C)(C)C</smiles>

$\underline{17}$

Schema 26: Oxidation der Verbindung $\underline{16}$

Dabei kristallisiert Verbindung $\underline{17}$ monoklin in der Raumgruppe Cc: 


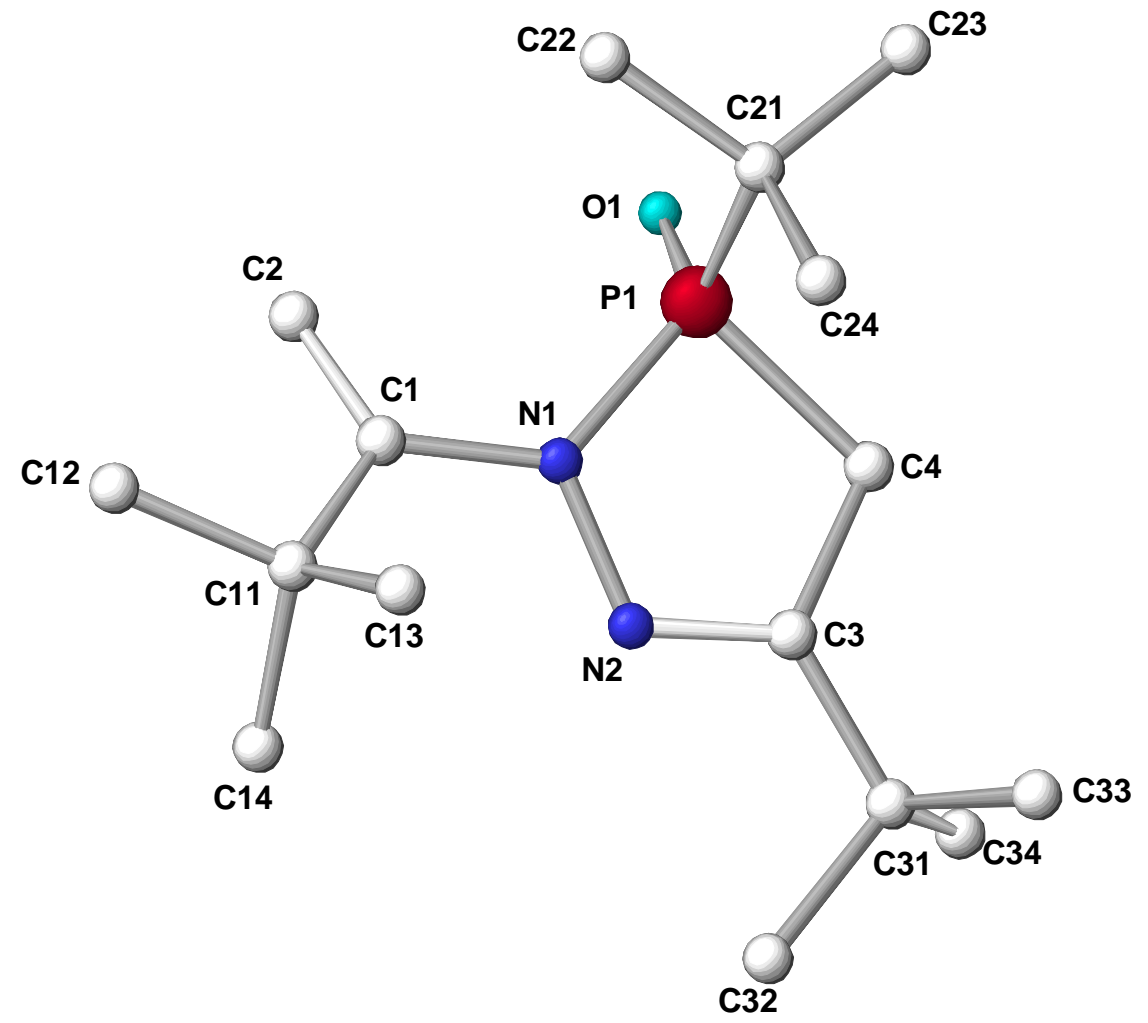

Abbildung 20: Struktur von Verbindung $1 \mathbf{1 7}$ (ohne Wasserstoffatome)

\begin{tabular}{|c|c|c|c|}
\hline$C(4)-C(3)$ & 148,3 & $\mathrm{C}(4)-\mathrm{C}(3)-\mathrm{N}(2)$ & 115,7 \\
\hline $\mathrm{C}(3)-\mathrm{N}(2)$ & 130,8 & $\mathrm{C}(3)-\mathrm{N}(2)-\mathrm{N}(1)$ & 114,8 \\
\hline$N(2)-N(1)$ & 137,0 & $N(2)-N(1)-P(1)$ & 114,0 \\
\hline$N(1)-P(1)$ & 171,7 & $N(2)-P(1)-C(4)$ & 90,5 \\
\hline$P(1)-C(4)$ & 183,9 & $P(1)-C(4)-C(3)$ & 105,0 \\
\hline$P(1)-O(1)$ & 138,3 & $\Sigma$ Innenwinkel & 540,0 \\
\hline
\end{tabular}

Tabelle 5: Ausgewählte Bindungslängen $(\mathrm{pm})$ und -winkel $\left({ }^{\circ}\right)$ von Verbindung $\underline{17}$

Alle Bindungslängen sind im Vergleich zu berechneten Einfach- und Mehrfachbindungen etwas verkürzt, entsprechen aber den zu erwartenden Abständen. Dabei weichen die $\mathrm{P}=\mathrm{O}-$ Doppelbindung mit $138 \mathrm{pm}$ und die $\mathrm{N}-\mathrm{N}$-Einfachbindung mit $137 \mathrm{pm}$ am stärksten von Literaturwerten ab $\left(\mathrm{P}=\mathrm{O}_{\text {ber. }}=148 \mathrm{pm}, \mathrm{N}-\mathrm{N}_{\text {ber. }}=148 \mathrm{pm}\right)^{57}$. Auch im Vergleich zu den fünfgliedrigen borhaltigen Ringen oder Teilringen unterscheiden sich diese beiden Werte am deutlichsten (NN entspricht $140 \mathrm{pm}$ (XV), $142 \mathrm{pm}(\mathbf{X}), 143 \mathrm{pm}(\underline{\mathbf{7}})$ und $150 \mathrm{pm}(\underline{\mathbf{5}}))$. Die PC-Bindungen stimmen mit $180 \mathrm{pm}$ bzW. 183 pm besser mit den berechneten Werten überein $\left(P-C_{\text {ber. }}=187\right.$ pm). 
Die Innenwinkelsumme des fünfgliedrigen Rings entspricht mit $540^{\circ}$ der Innenwinkelsumme eines geometrischen Fünfecks. Dabei wird von den $\mathrm{P}(1)-\mathrm{C}(4)-\mathrm{C}(3)-\mathrm{N}(2)-$ $\mathrm{N}(1)$-Atomen eine Ebene aufgespannt, die innerhalb von 0,9 pm koplanar ist. Eine Seitenansicht der Ringebene von Verbindung $\underline{17}$ verdeutlicht dies:

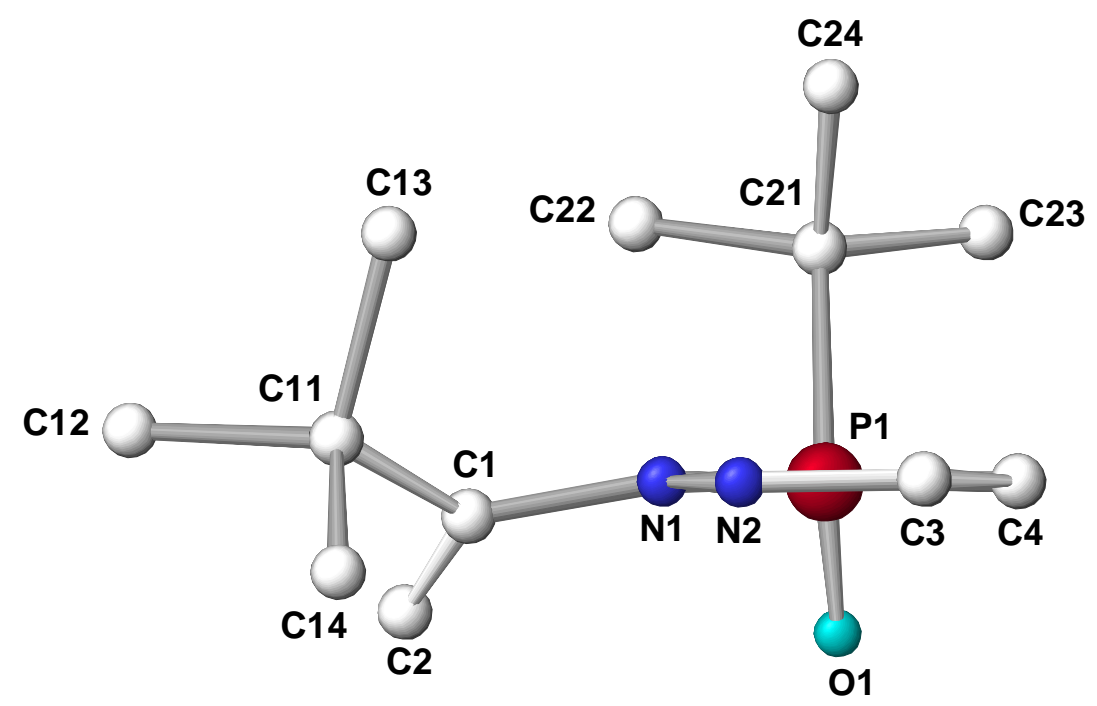

Abbildung 21: Seitenansicht der Ringebene von Verbindung 17 (ohne Substituent an $\mathrm{C}(4)$ )

Das Phosphoratom weist mit einer Winkelsumme von $653,3^{\circ}$ eine fast tetraedrische Geometrie auf, wobei der Ringinnenwinkel mit $90^{\circ}$ am stärksten verkleinert und der $\mathrm{N}(1)-\mathrm{P}(1)-\mathrm{O}(1)-$ Winkel mit $120^{\circ}$ am deutlichsten vergrößert ist. Beide Winkel weisen auf nicht unerhebliche Anteile von d-Orbitalen in den Bindungen hin. Die Winkelsumme des $\mathrm{N}(1)$ weicht mit $358,7^{\circ}$ etwas von der idealen trigonal-planaren Konfiguration ab. Dies lässt sich auf die sterischen Ansprüche des tert.-Butylsubstituenten am Phosphoratom zurückführen, der gleichzeitig auch für die Torsion der $\mathrm{C}(2)-\mathrm{C}(1)-\mathrm{N}(1)-\mathrm{N}(2)-K e t t e$ um $145^{\circ}$ verantwortlich ist. 


\subsubsection{Hydrolyse des 3,5-Di-tert.-butyl-2-(3,3-dimethylbut-1-en-2-yl)-1,2-diaza- 3-phosphacyclopent-5-ens $\underline{16}$}

Bei gezielter Umsetzung des Cyclopentens $\underline{16}$ mit einem Moläquivalent Wasser, aber auch schon bei Lagerung ohne trockenes Inertgas im geschlossenen Gefäß, entsteht das Hydrolyseprodukt $\underline{\mathbf{1 8}}$ :

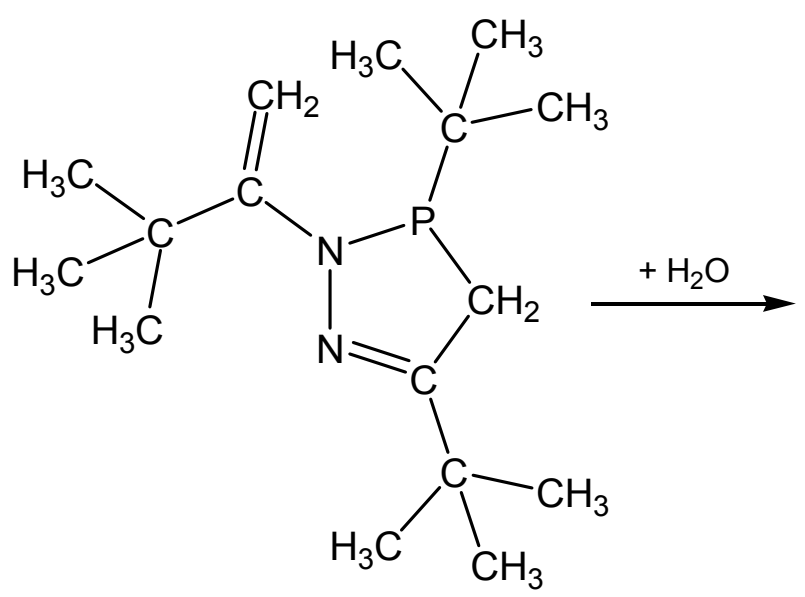

$\underline{16}$<smiles>C/C(=N\N=C(/C[P+](=O)C(C)(C)C)C(C)(C)C)C(C)(C)C</smiles>

$\underline{18}$

Schema 27: Hydrolyse der Verbindung $\underline{16}$

Dabei wird die PN-Bindung gelöst und ein acyclisches Ketazingerüst ausgebildet. Verbindung $\underline{18}$ ist ein helles, fast weißes, mikrokristallines Pulver, das sich gut in polaren Lösungsmitteln löst. Die Strukturaufklärung erfolgte über ${ }^{1} \mathrm{H}-,{ }^{13} \mathrm{C}$ - und ${ }^{31} \mathrm{P}$ NMR-Spektroskopie. Dabei erleichterte das Kopplungsverhalten des Phosphorkerns die Zuordnung aller Signale. Die Methylgruppe liefert im ${ }^{1} \mathrm{H}-\mathrm{NMR}-S p e k t r u m$ ein Singulett bei 1,90 ppm, während jedes Proton der Methylengruppe zu einem Dublett von Dublett von Dublett aufspaltet. Dabei konnten $\mathrm{H}_{\mathrm{A}}$ die Kopplungskonstanten ${ }^{2} \mathrm{~J}_{\mathrm{H}_{A} \mathrm{H}_{B}}=$ $13,1 \mathrm{~Hz},{ }^{2} \mathrm{~J}_{\mathrm{H}_{\mathrm{A}} \mathrm{P}}=17,8 \mathrm{~Hz}$ und ${ }^{3} \mathrm{~J}_{\mathrm{H}_{\mathrm{A}} \mathrm{H}_{\mathrm{P}}}=7,8 \mathrm{~Hz}$ zugeordnet werden. Für das $\mathrm{H}_{\mathrm{B}}$ der Methylengruppe wurde eine ${ }^{2} \mathrm{~J}_{\mathrm{H}_{B} \mathrm{H}_{\mathrm{A}}}-$ Kopplung von $13,1 \mathrm{~Hz}$, eine ${ }^{2} \mathrm{~J}_{\mathrm{H}_{B} \mathrm{P}-K o p p l u n g}$ von

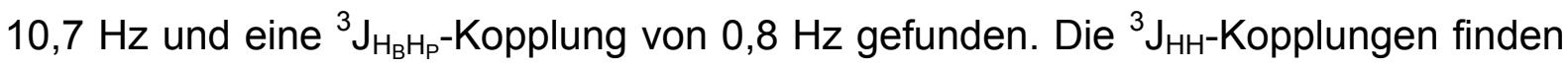
sich im Signal des am Phosphoratom sitzenden Wasserstoffkerns $\mathrm{H}_{\mathrm{P}}$ wieder. Außerdem weist dieser noch die ${ }^{1} \mathrm{~J}_{\mathrm{H}_{\mathrm{P}} \mathrm{P}}$-Kopplung von $463 \mathrm{~Hz}$ auf. Für eine mögliche $\mathrm{NH}-$ Gruppierung gibt es keine Anzeichen. Das Integral des ${ }^{1} \mathrm{H}-\mathrm{NMR}-S p e k t r u m s$ bestätigt die eindeutige Zuweisung der oben aufgeführten Methyl- und Methylen-Signale. 
Es ist möglich, dass es beim Lösen der PN-Bindung, neben der Ausbildung der $\mathrm{P}=\mathrm{O}$-Doppelbindung und der $\mathrm{PH}-$ Bindung, zur Bildung einer $\mathrm{NH}$-Gruppe kommt. Im NMR-Spektrum wird dieses N1-Butyliden-N1-Wasserstoff-Hydrazon jedoch nicht gefunden. Über die Imin-Enamin-Tautomerie könnte sich ein intermediäres Hydrazon zu dem konjugierten Doppelbindungssystem des Ketazins stabilisieren:<smiles>C=C(NN=C(C[P](=O)C(C)(C)C)C(C)(C)C)C(C)(C)C</smiles>

Enamin<smiles>C/C(=N\N=C(/C[P+](=O)C(C)(C)C)C(C)(C)C)C(C)(C)C</smiles>

Imin

18

Schema 28: Enamin-Tautomer als mögliches Übergangsprodukt bei der Bildung von Verbindung $\underline{18}$

Setzt man die Verbindungen $\underline{16}$ oder $\underline{18}$ über längere Zeit einer feuchten Stickstoffatmosphäre aus, oder gibt Wasser im Überschuss hinzu, hydrolysieren beide Verbindungen vollständig zu Ketazin VIII und phosphoriger Säure. Bei gleichzeitiger Anwesenheit von Feuchtigkeit und Sauerstoff bildet sich als Zersetzungsprodukt neben dem Bis(tert.-butyl-methyl)ketazin Phosphorsäure. Die Identifizierung ist durch NMRMessungen leicht möglich.

\subsubsection{Darstellung des 5-tert.-Butyl-2-(3,3-dimethylbut-1-en-2-yl)-3-phenyl-1,2- diaza-3-phosphacyclopent-5-ens $\underline{19}$}

Nach gelungener Synthese eines P-alkylierten Diazaphosphacyclopentens sollte die Darstellung eines arylsubstituierten Rings auf ähnliche Weise möglich sein. Nach Zugabe von Dichlorphenylphosphan zu dem dilithiierten Ketazin VIII erhält man das P-Phenyl-Diazaphosphacyclopenten 19: 


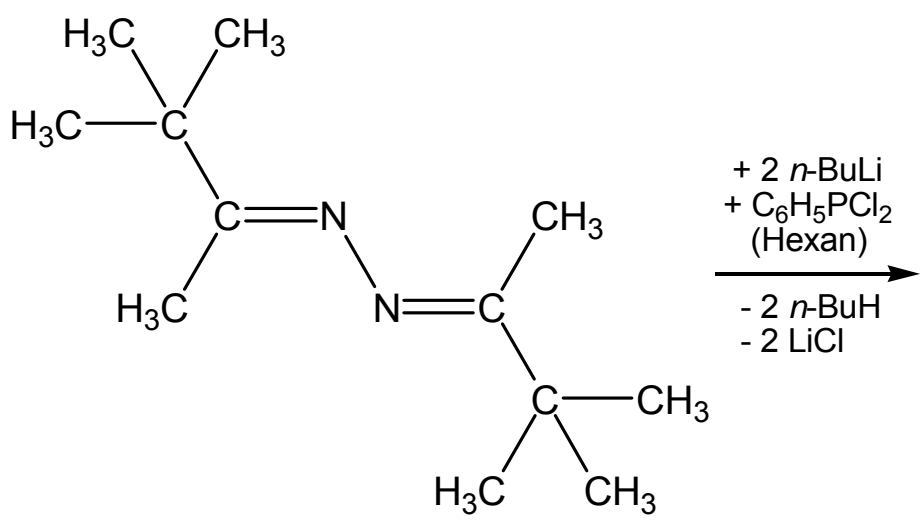

VIII<smiles>C=C(N1N=C(C(C)(C)C)CP1c1ccccc1)C(C)(C)C</smiles>

$\underline{19}$

Schema 29: Darstellung von Verbindung $\underline{19}$

Verbindung $\underline{19}$ ist wie Verbindung $\underline{16}$ ein heller Feststoff, der aber deutlich weniger hydrolyse- und oxidationsempfindlich ist und sich unter Inertgasatmosphäre mehrere Monate lagern lässt. Bei einer gezielten Hydrolyse kann kein Produkt analog zu $\underline{16}$ isoliert werden. Das Diazaphosphacyclopenten $\underline{19}$ hydrolysiert vollständig zu phosphoriger Säure und Azin.

\subsubsection{Versuchte Darstellung eines acyclischen Phosphylketazins}

Bei den bisherigen Umsetzungen gelang die Darstellung acyclischer Verbindungen nicht. Wie in Kapitel 3.1.2.8 beschrieben, führte die Umsetzung eines Aminodifluorborans mit dem Bis(tert.-butyl-methyl)ketazin VIII im Arbeitskreis MELLER zur Ausbildung einer Kettenstruktur. Daher ist die Reaktion eines Aminodifluorphosphans mit einem Ketazinderivat vielversprechend. Gibt man zu dem monolithiierten Ketazin IX in $n$-Hexan (Diisopropylamino)difluorphosphan, so kann kein acyclisches Produkt isoliert werden, sondern das Diazaphosphacyclopenten 21: 


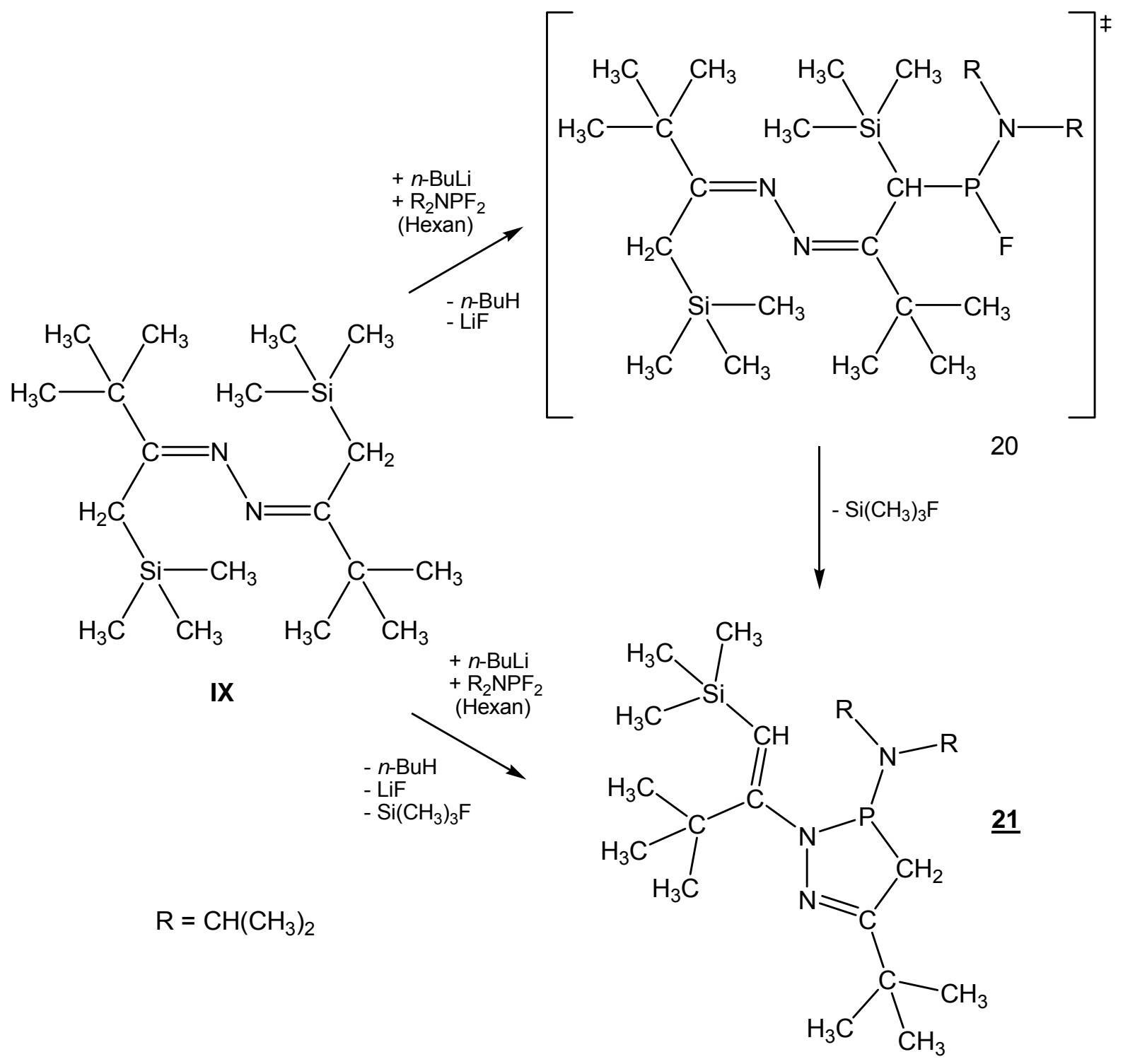

Schema 30: Umsetzung des monolithiierten Ketazins IX mit $\left(\mathrm{iPr}_{2} \mathrm{~N}\right) \mathrm{PF}_{2}$

Spätestens während der thermischen Aufarbeitung des Reaktionsgemischs spaltet das oben vorgestellte acyclische Zwischenprodukt 20 ein Molekül Fluortrimethylsilan ab und bildet den gelben Feststoff $\underline{\mathbf{2 1}}$. ${ }^{31}$ P-NMR-Messungen der Reaktionslösung zeigen kurz nach Zugabe des Phosphans zu dem Lithiumsalz drei Phosphorsignale. Davon lässt sich das eine dem Aminophosphan und das andere dem Produkt 21 zuordnen. Es ist anzunehmen, dass es sich bei dem dritten Signal um das des Zwischenprodukts 20 handelt.

Die Trimethylsilylgruppe in Nachbarschaft zu dem Fluoratom stellt eine gute Abgangsgruppe dar. Umsetzungen des einfach metallierten unsilylierten Ketazins VIII 
mit Aminophosphanen sind noch nicht untersucht. Hierbei könnte eine intramolekulare Cyclisierung durch Fluortrimethylsilylabspaltung unterbunden werden.

Verbindung $\underline{\mathbf{2 1}}$ kristallisiert monoklin mit der Raumgruppe P2(1)/c:

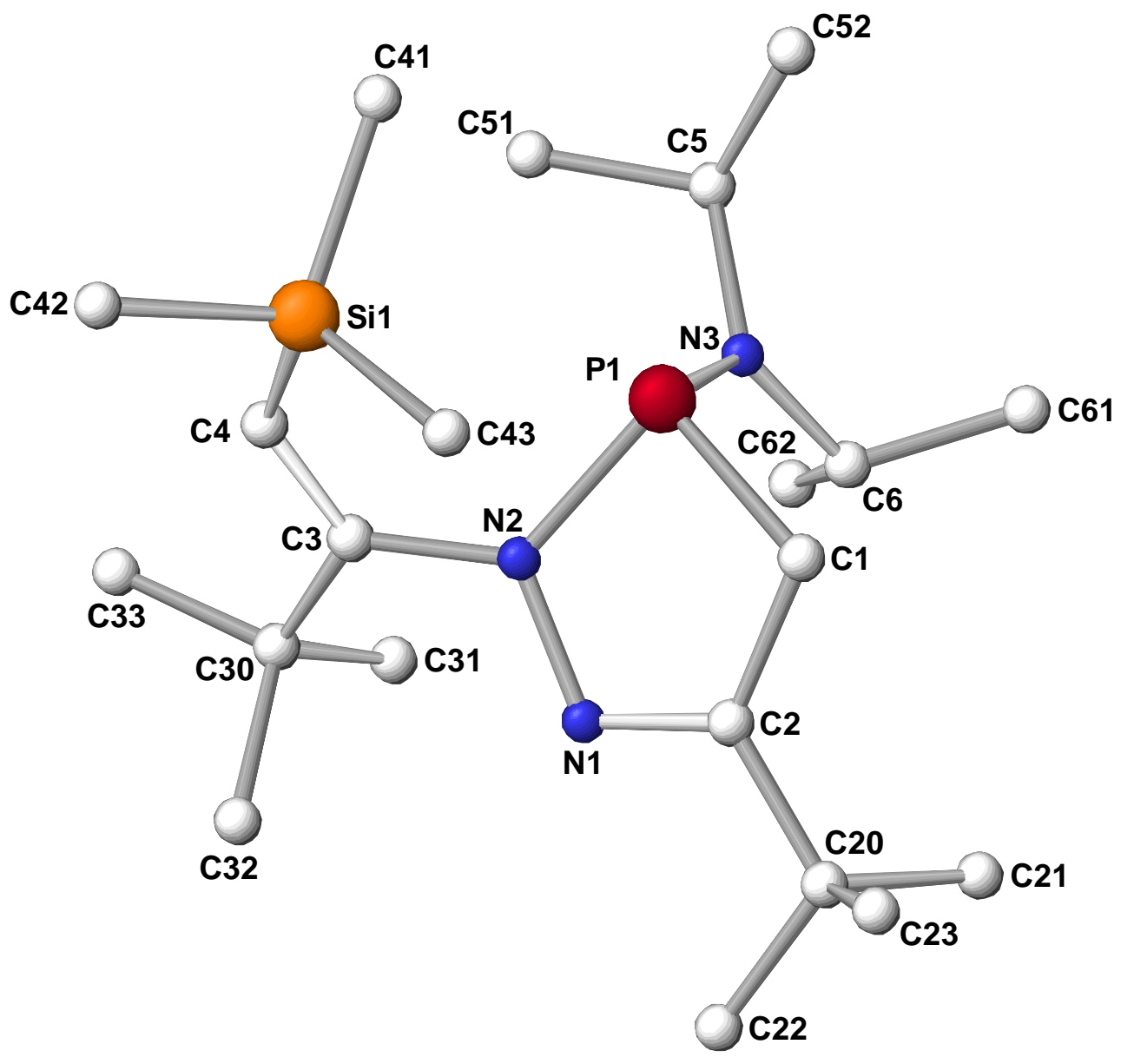

Abbildung 22: Struktur von Verbindung $\underline{21}$ (ohne Wasserstoffatome)

\begin{tabular}{|ll||cc|}
$\mathrm{C}(1)-\mathrm{C}(2)$ & 151,4 \\
$\mathrm{C}(2)-\mathrm{N}(1)$ & 127,7 \\
$\mathrm{~N}(1)-\mathrm{N}(2)$ & 141,4 \\
$\mathrm{~N}(2)-\mathrm{P}(1)$ & 175,1 \\
$\mathrm{P}(1)-\mathrm{C}(1)$ & 184,6 \\
$\mathrm{P}(1)-\mathrm{N}(3)$ & 168,2 & $\mathrm{C}(1)-\mathrm{C}(2)-\mathrm{N}(1)$ & 116,4 \\
$\mathrm{C}(2)-\mathrm{N}(1)-\mathrm{N}(2)$ & 110,9 \\
$\mathrm{~N}(1)-\mathrm{N}(2)-\mathrm{P}(1)$ & 116,1 \\
$\mathrm{~N}(2)-\mathrm{P}(1)-\mathrm{C}(1)$ & 86,1 \\
$\mathrm{P}(1)-\mathrm{C}(1)-\mathrm{C}(2)$ & 105,9 \\
& & I Innenwinkel & 535,4
\end{tabular}

Tabelle 6: Ausgewählte Bindungslängen $(\mathrm{pm})$ und -winkel $\left({ }^{\circ}\right)$ von Verbindung $\underline{14}$

Alle Bindungslängen liegen in den erwarteten Bereichen für P-N-, P-C-, Si-C-, C-Noder C-C-Einfach-, bzw. für $\mathrm{C}=\mathrm{C}$ - und $\mathrm{C}=\mathrm{N}-$ Doppelbindungen ${ }^{57}$. Die exocyclische PN-Bindung ist allerdings gegenüber der endocyclischen verkürzt. 
Die Summe der Innenwinkel des fünfgliedrigen Rings weicht mit 535,5 pm schon deutlich von der eines geometrischen Fünfecks ab. Das Phosphoratom steht um 43,2 pm aus der von den Atomen $\mathrm{C}(1)-\mathrm{C}(2)-\mathrm{N}(1)-\mathrm{N}(2)$ beschriebenen Ebene heraus. Der Ring ist nicht mehr planar. Die Winkelsumme von $359,6^{\circ}$ an $\mathrm{N}(2)$ zeigt die $\mathrm{sp}^{2}-$ Hybridisierung des Stickstoffatoms an. Die C(4)-C(3)-N(2)-N(1)-Kette ist um $141,1^{\circ}$ tordiert, das $\mathrm{Si}(1)-\mathrm{C}(4)-\mathrm{C}(3)-\mathrm{N}(2)-G e r u ̈ s t ~ u m ~ d i e ~ C=C-D o p p e l b i n d u n g ~ u m ~ 18,1^{\circ}$. Die sterische Behinderung der Trimethylsilylgruppe durch den P-Aminosubstituenten wird dadurch minimiert:

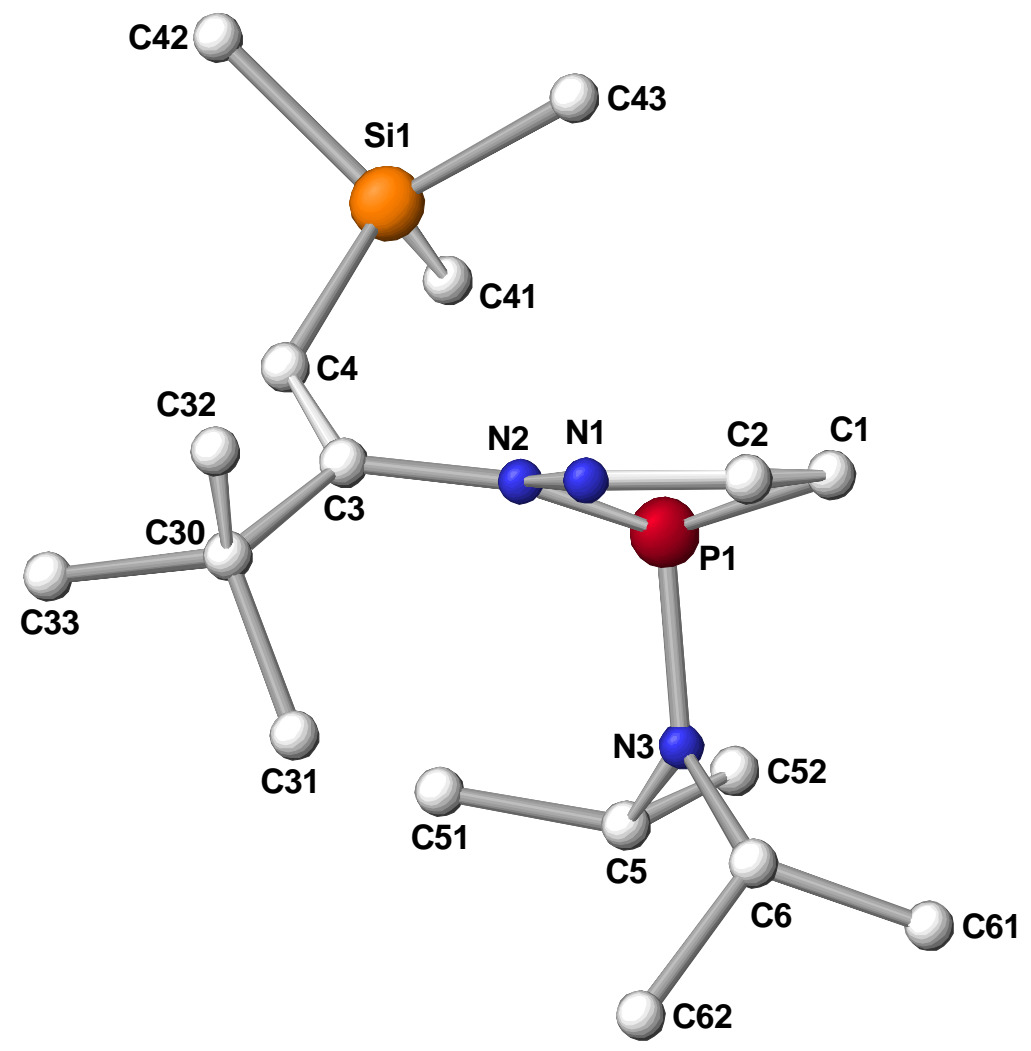

Abbildung 23: Seitenansicht der Ringebene von Verbindung 21 (ohne Alkylsubstituent an $\mathrm{C}(2)$ )

Die Winkel am Phosphoratom sind mit N(2)-P(1)-N(3) $=109,3^{\circ}, N(2)-P(1)-C(1)=$ $86,1^{\circ}$ und $\mathrm{N}(3)-\mathrm{P}(1)-\mathrm{C}(1)=103,6^{\circ}$ nicht mit denen aus Verbindung $\mathbf{X}$ vergleichbar. Das vierfach koordinierte Boratom besitzt eine tetraedrische Molekülgeometrie. Verbindung XV bietet sich wegen der dreifachen Koordination des Boratoms als Vergleich an. Allerdings weichen die analogen Winkel am Boratom mit N(8)-B(7)-O(7) = $118,8, \mathrm{~N}(8)-\mathrm{B}(7)-\mathrm{C}(6)=101,8$ und $\mathrm{O}(7)-\mathrm{B}(7)-\mathrm{C}(6)=139,5^{\circ}$ deutlich von denen am 
Phosphoratom in $\underline{\mathbf{2 1}}$ ab. Die Winkelsumme von 360,1 $1^{\circ}$ zeigt die trigonal-planare Geometrie und die $\mathrm{sp}^{2}$-Hybridisierung des Boratoms in Verbindung XV.

Die deutlichen Unterschiede in den Bindungswinkeln liegen zum einen an den längeren Phosphorbindungen - gerade bei dem Ringinnenwinkel, und zum anderen an der wenig ausgeprägten Hybridisierung der Phosphororbitale. Alle $\sigma$-Bindungen werden vorwiegend von den p-Orbitalen des Phosphors ausgebildet, die in einem $90^{\circ}$-Winkel zueinander stehen. Nur der N-P-N-Winkel entspricht einem Tetraederwinkel und weist auf eine zumindest teilweise Hybridisierung der Phosphororbitale hin.

\subsubsection{Synthese achtgliedriger phosphorhaltiger Ringe}

Wie in der Bor-Azin-Chemie entstehen in der Phosphor-Azin-Chemie neben fünfgliedrigen Ringen auch achtgliedrige Bicyclen. Bei der Darstellung von $\underline{13}$ wird der Bicyclus $\underline{14}$ als Nebenprodukt erhalten. Bei Umsetzungen von Ketazin VIII mit substituierten Phosphanen konnten keine achtgliedrigen Ringe isoliert werden. Die Halogenfunktion des 4,8-Di-tert.-butyl-2,6-dichlor-1,5-diaza-2,6-diphospha-bicyclo[3.3.0]octa-3,7-diens $\underline{14}$ bietet jedoch die Möglichkeit der Substitution.

\subsubsection{Kristallstruktur des 4,8-Di-tert.-butyl-2,6-dichlor-1,5-diaza-2,6- diphospha-bicyclo[3.3.0]octa-3,7-diens $\underline{14}$}

Einkristalle von Verbindung $\underline{14}$ wurden durch Umkristallisation in $n$-Hexan gewonnen. Sie kristallisieren monoklin mit der Raumgruppe $P 2(1) / n$ und sind farblos. Als Feststoff ist Verbindung $\underline{14}$ unter Inertgasatmosphäre problemlos mehrere Wochen stabil. Das Molekül zeigt im Festkörper in Bezug auf die Chlorsubstituenten der Phosphoratome eine wannenähnliche Konformation: 


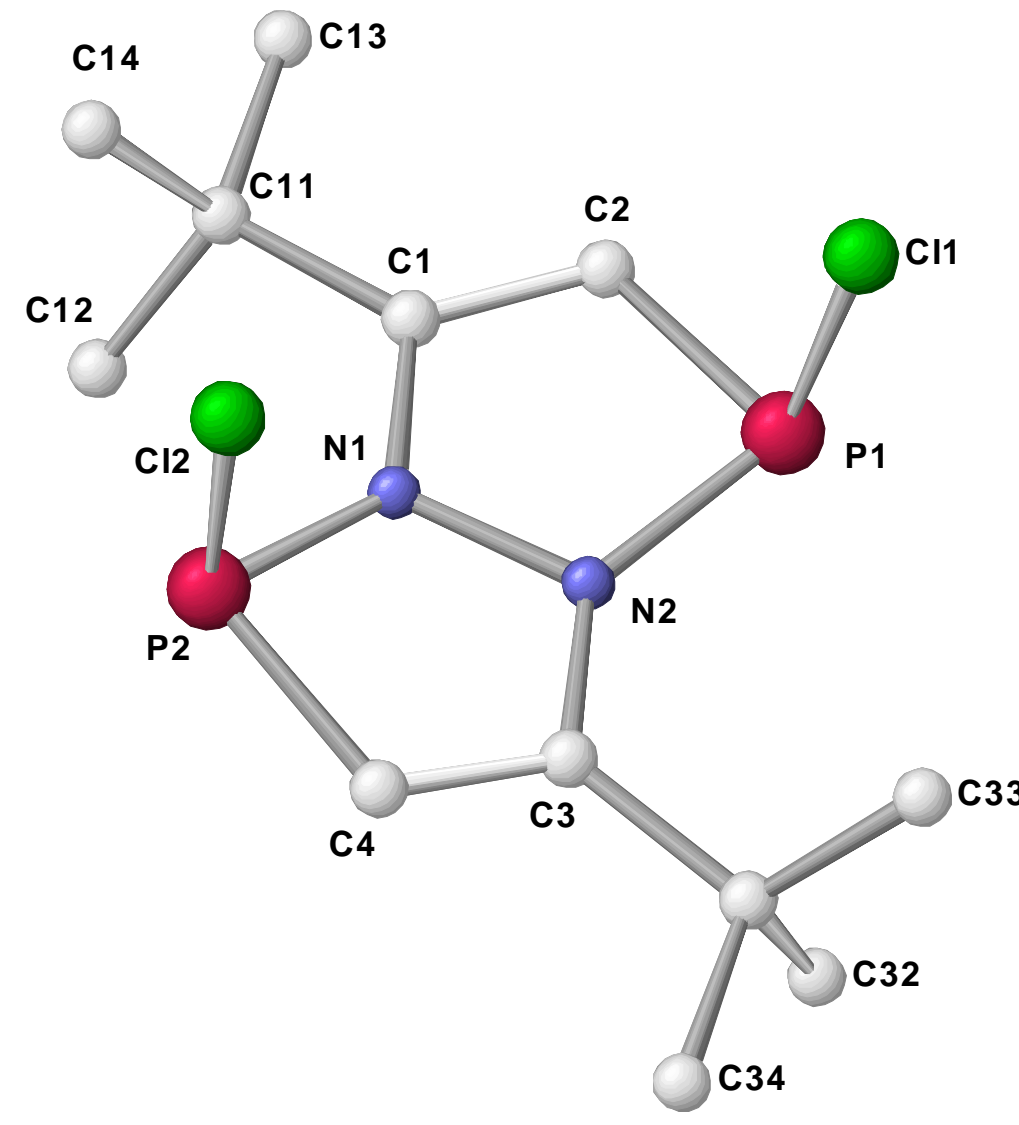

Abbildung 24: Struktur von Verbindung $\underline{14}$ (ohne Wasserstoffatome)

\begin{tabular}{|c|c|c|c|}
\hline$C(1)-C(2)$ & 135,3 & $C(1)-C(2)-P(1)$ & 113,2 \\
\hline$C(2)-P(1)$ & 177,1 & $C(2)-P(1)-N(2)$ & 88,9 \\
\hline$P(1)-N(2)$ & 169,8 & $P(1)-N(2)-N(1)$ & 113,2 \\
\hline$N(2)-N(1)$ & 142,6 & $N(2)-N(1)-C(1)$ & 110,7 \\
\hline$N(1)-C(1)$ & 139,9 & $N(1)-C(1)-C(2)$ & 112,7 \\
\hline $\mathrm{P}(1)-\mathrm{Cl}(1)$ & 213,8 & $\Sigma$ Innenwinkel & 538,7 \\
\hline
\end{tabular}

Tabelle 7: Ausgewählte Bindungslängen $(\mathrm{pm})$ und -winkel $\left(^{\circ}\right)$ von Verbindung $\underline{14}$

Das Ketazingerüst des Doppelrings ist nahezu identisch zu der borhaltigen Verbindung XV, die in Kapitel 3.1.3 beschrieben wurde. Die Bindungslängen $N(1)-C(1)$ und $C(1)-C(2)$ stimmen bis auf $0,3 \mathrm{pm}$ mit denen der Verbindung $X V$ überein. Nur die NNBindung ist im Vergleich bei $\underline{\mathbf{1 4}}$ um 2,6 pm länger. Es liegen also charakteristische C$\mathrm{N}$-Einfach- und $\mathrm{C}=\mathrm{C}$-Doppelbindungen vor. Die Bindungen des Phosphoratoms entsprechen jeweils Einfachbindungen, die berechnet mit $\mathrm{P}-\mathrm{N}=176 \mathrm{pm}, \mathrm{P}-\mathrm{C}=187 \mathrm{pm}$, und $\mathrm{P}-\mathrm{Cl}=204 \mathrm{pm}$ angegeben werden ${ }^{57}$. Die endocyclischen Bindungen sind verkürzt, während die exocyclische Bindung verlängert ist. 
Die Bindungswinkel lassen sich wegen des unterschiedlichen Heterokerns in den Ringen nicht direkt mit denen von Verbindung XV vergleichen (siehe auch Kapitel 3.2.2). Der $\mathrm{N}(2)-\mathrm{P}(1)-\mathrm{C}(2)-W i n k e l$ ist mit $88,9^{\circ}$ im Vergleich zu dem entsprechenden Winkel in Verbindung XV $\left(\mathrm{N}(4)-\mathrm{B}(3)-\mathrm{C}(2)=103,8^{\circ}\right)$ deutlich kleiner. Damit entsprechen die Bindungsverhältnisse im Bicyclus $\underline{14}$ am ehesten denen der Verbindung 21 (14: N-P-Cl 105 ${ }^{\circ}, \mathrm{C}-\mathrm{P}-\mathrm{Cl} \sim 100^{\circ}, \mathbf{2 1}$ : N-P-N' 109 ${ }^{\circ}, \mathrm{C}-\mathrm{P}-\mathrm{N}^{\prime} \sim 104^{\circ}$ ). Die Innenwinkelsummen der fünfgliedrigen Teilringe kommen mit rund $538,5^{\circ}$ denen eines geometrischen Fünfecks nahe. Allerdings ragen die Phosphoratome aus der von den $\mathrm{C}(1)-\mathrm{N}(1)-\mathrm{N}(2)-\mathrm{C}(3)-A t o m e n$ aufgespannten Ebene um etwa $31 \mathrm{pm}$ in dieselbe Richtung heraus. $\mathrm{C}(2)$ und $\mathrm{C}(4)$ stehen um etwa 13 pm von dieser Ebene ab. Die nachfolgende Abbildung verdeutlicht dies:

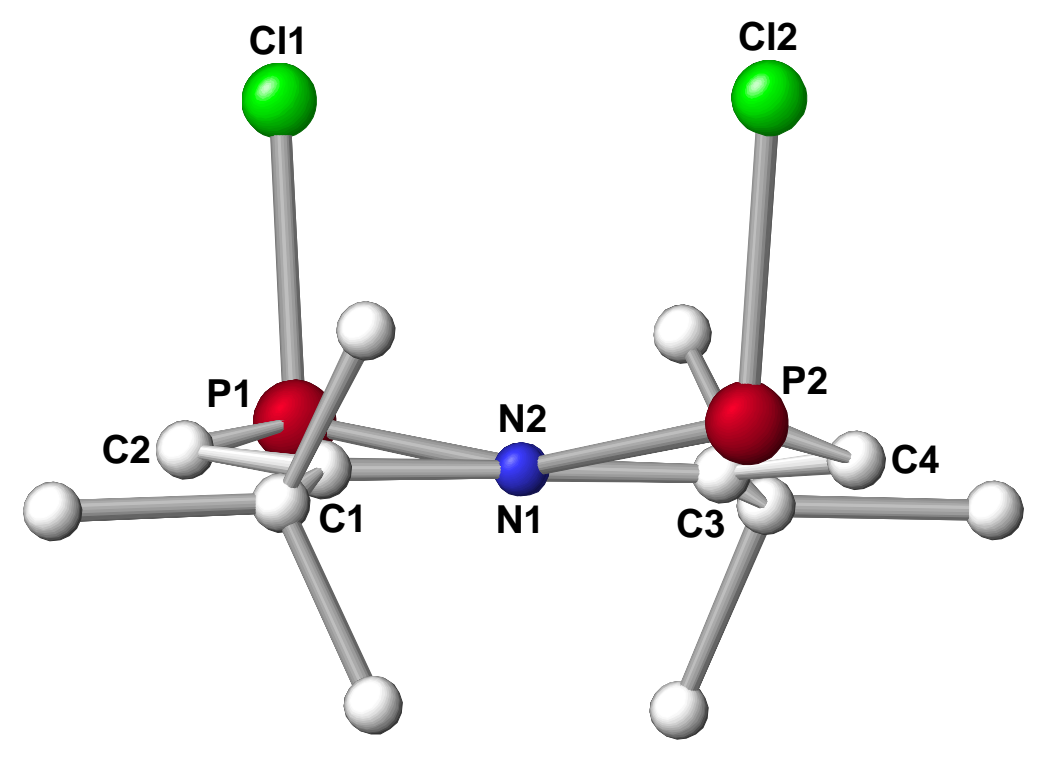

Abbildung 25: Blick auf die NN-Bindungsachse von Verbindung $\underline{14}$

Wegen der ähnlichen Bindungsverhältnisse im Ketazingerüst bietet sich der Vergleich mit Verbindung XV an:

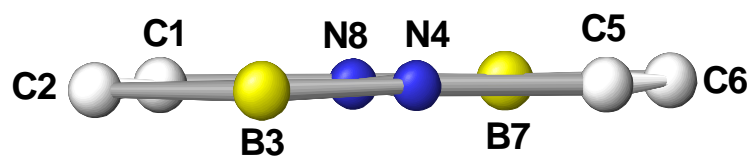

XV

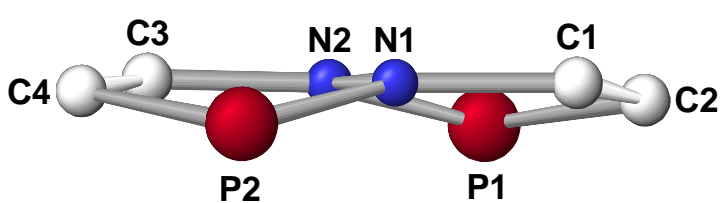

$\underline{14}$

Abbildung 26: Vergleich der Seitenansichten der Verbindungen XV und $\underline{14}$ 


\subsubsection{Substitution von 4,8-Di-tert.-butyl-2,6-dichlor-1,5-diaza-2,6-diphospha- bicyclo[3.3.0]octa-3,7-dien $\underline{14}$}

Substitutionsversuche mit Lithiumorganylen verlaufen nicht wie erwartet. Zu der in $n$-Hexan gelösten Verbindung $\underline{14}$ werden in verschiedenen Umsetzungen tert.-Butyl-, Phenyl- und Methyllithium bzw. Lithium-(bis(trimethylsilyl)amid) im Verhältnis 2:1 zugegeben. Dabei entstehen Produktgemische, die bis auf die Reaktion mit Methyllithium nicht zu isolierbaren Verbindungen führen.

Eine Reaktion mit Methyllithium liefert das Produkt 23:
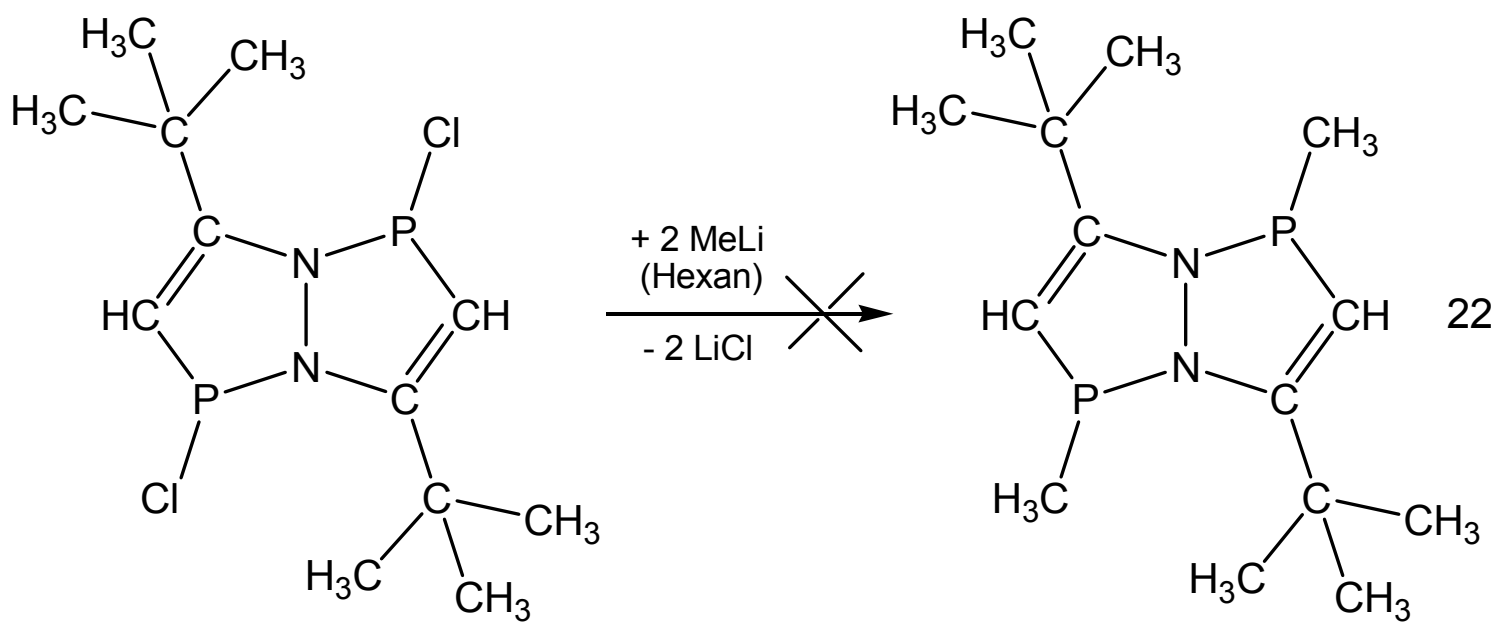

$\underline{14}$
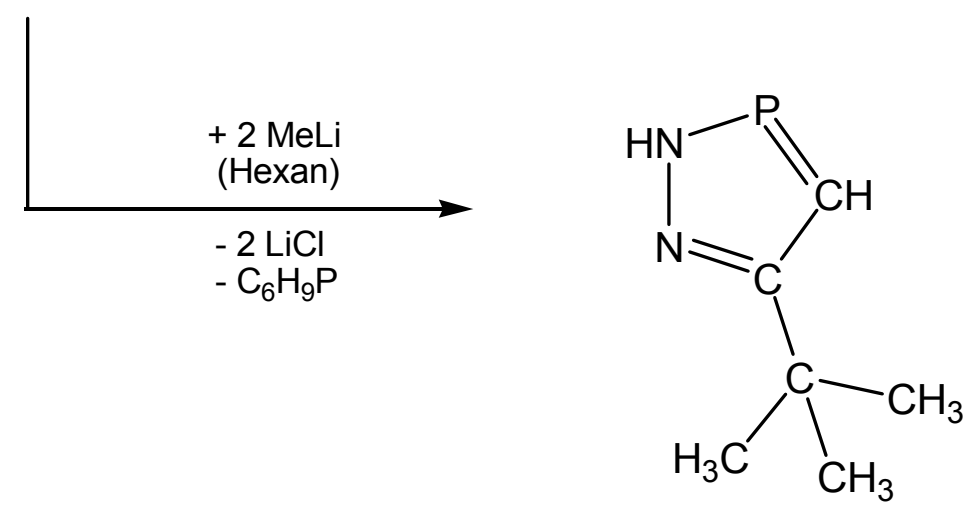

$\underline{23}$

Schema 31: Umsetzung der Verbindung $\underline{14}$ mit Methyllithium

Verbindung $\underline{23}$ wird dabei zu etwa $15 \%$ erhalten. Vorwiegend reagieren die Lithiumorganyle also nicht als Substitutionsreagenzien, sondern als starke Basen. Aus dem Bicyclus $\underline{14}$ entsteht nach einem noch ungeklärten Reaktionsmechanismus das stabile Phosphol 23. Massenspektren zeigen, dass ein Butyliden-Rest von einem Diazaphosphacyclopenten abgespalten werden kann $\left([\mathrm{M}]^{+}=83 \mathrm{u}\right)$. Unter moderaten Bedingungen ist eine Substitution des Butylidensubstituenten durch ein Wasserstoff- 
atom nicht bekannt, da der Abspaltung des N-Butyliden eine Protonenumlagerung oder eine Hydridionenwanderung folgen müsste.

Auf anderem Wege gelang die Synthese von Verbindung $\underline{23}$ durch Umsetzung des tert.-Butyl-methyl-hydrazons XVI mit Trichlorphosphan in Toluol:<smiles>C/C(=N\N)C(C)(C)C</smiles>

$\mathrm{XVI}$

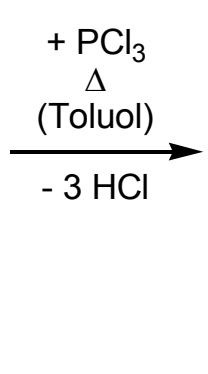

Es stellt sich die Frage, ob die Basen nicht beide P-N-Einfachbindungen spalten und sich im alkalischen Milieu ein Gleichgewicht zwischen Ketazin und Hydrazon einstellt (vgl. Kapitel 1.1). Eine anschließende Bildung des stabilen fünfgliedrigen Rings während der thermischen Aufarbeitung ist denkbar.

Dies könnte auch die große Anzahl von Nebenreaktionen und die unerwarteten Produkte bei den Umsetzungen mit tert.-Butyl- und Phenyllithium erklären.

Eine versuchte Oxidation des Bicyclus $\underline{14}$ mit Schwefel führte zu keinem Ergebnis.

\subsubsection{Reduktion des 4,8-Di-tert.-butyl-2,6-dichlor-1,5-diaza-2,6-diphospha- bicyclo[3.3.0]octa-3,7-diens $\underline{14}$}

Durch eine Reduktion sollte es möglich sein, die Verbindung $\underline{14}$ von einem bicyclischen Octadien in einen achtgliedrigen Ring mit vier alternierenden Doppelbindungen umzuwandeln: 
<smiles>CC(C)(C)C1=CP(Cl)N2C(C(C)(C)C)=CP(Cl)N12</smiles>

$\underline{15}$

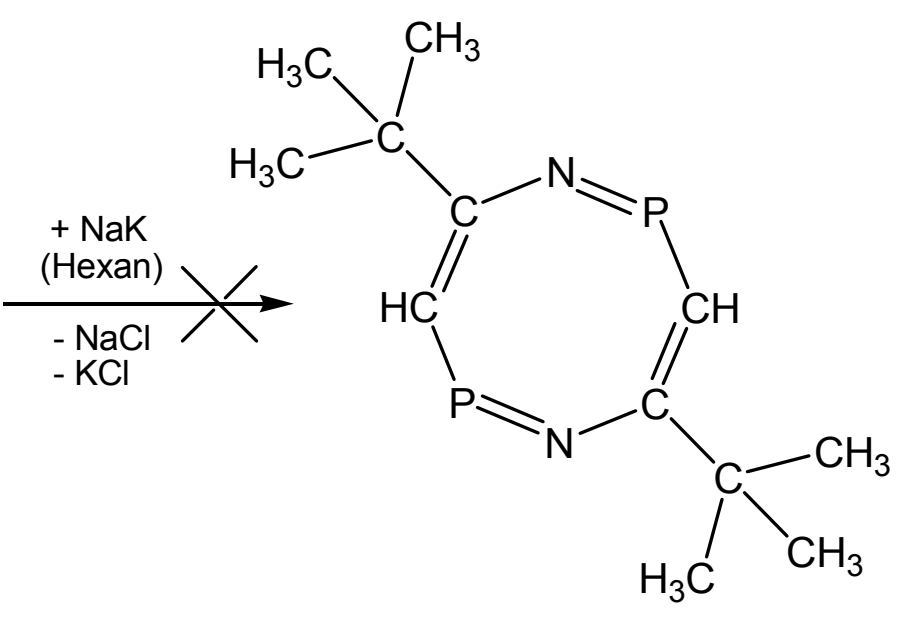

24

Schema 33: Mögliche Reduktion von Verbindung 14

Ein solcher Ring wäre für Komplexe mit Übergangsmetallen interessant. Verbindung 24 lässt sich bei der Reaktion des Bicyclus $\underline{14}$ mit zwei Moläquivalenten Natrium-Kalium-Legierung nicht isolieren. Aus der Reaktionslösung kristallisiert Verbindung $\underline{25}$ mit einer Ausbeute unter $5 \%$.<smiles>CC(C)(C)C1=CP(Cl)N2C(C(C)(C)C)=CP(Cl)N12</smiles>

$\underline{14}$
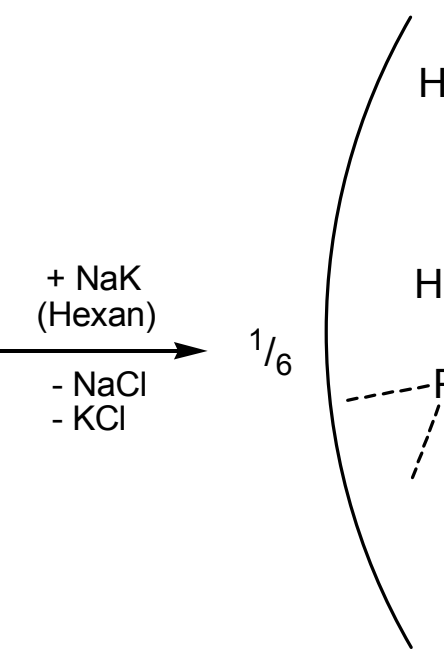

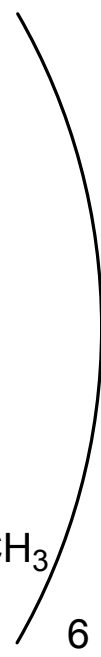

$\underline{25}$

Schema 34: Umsetzung der Verbindung 14 mit NaK-Legierung

Verbindung $\underline{25}$ ist ein Cyclohexaphosphan, das an jedem Phosphoratom über den Butylidenrest mit einem Molekül $\underline{13}$ substituiert ist.

Bisherige Darstellungen von Cyclophosphanen verliefen z.B. über Aryldifluorphosphane, die in Chloroform disproportionierten ${ }^{44}$. 
Ein möglicher Reaktionsmechanismus zur Bildung des Cyclophosphans ist in Schema 35 vorgestellt:

6<smiles>CC(C)(C)C1=CP(Cl)N2C(C(C)(C)C)=CP(Cl)N12</smiles>

\section{$\underline{14}$}

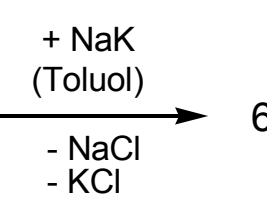

$-\mathrm{KCl}$

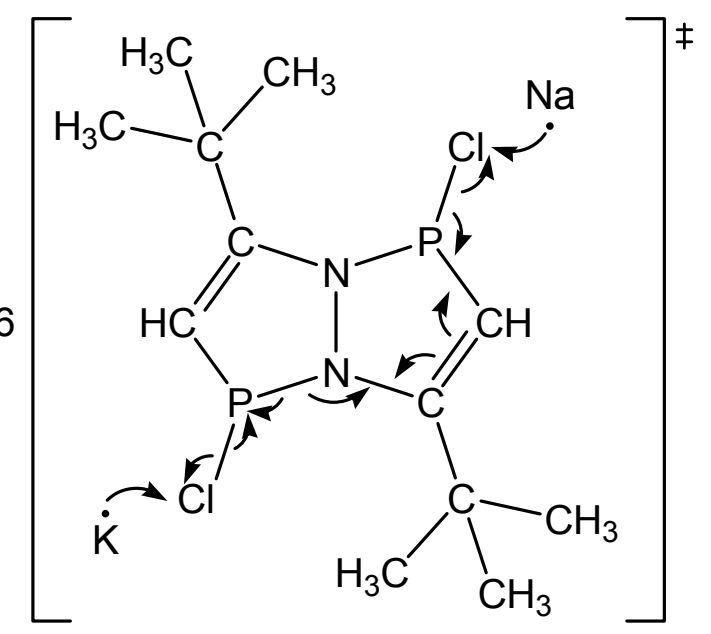<smiles>[3H][V]</smiles><smiles>CP(C)/C=C(/n1nc(C(C)(C)C)cp1)C(C)(C)C</smiles><smiles>[CH2]C(C)(C)c1cpn(/C(=C/P)C(C)(C)C)n1</smiles>

$\underline{25}$

Schema 35: Mögliche Bildung von Verbindung $\underline{25}$

Untersuchungen zum Mechanismus stehen noch aus. Der formulierte diradikalische Übergangszustand soll der Anschauung dienen. Ein paralleler Aufbau der PPBindungen während der Spaltung der PCl-Bindung ist aber wahrscheinlicher. Die Bildung einer P-P-Einfachbindung ist auch schon in anderen Fällen als treibende Kraft einer Reaktion beschrieben worden.

Die Röntgenstrukturanalyse bestätigt den Molekülaufbau des Produkts $\underline{\mathbf{2 5}}$ : 


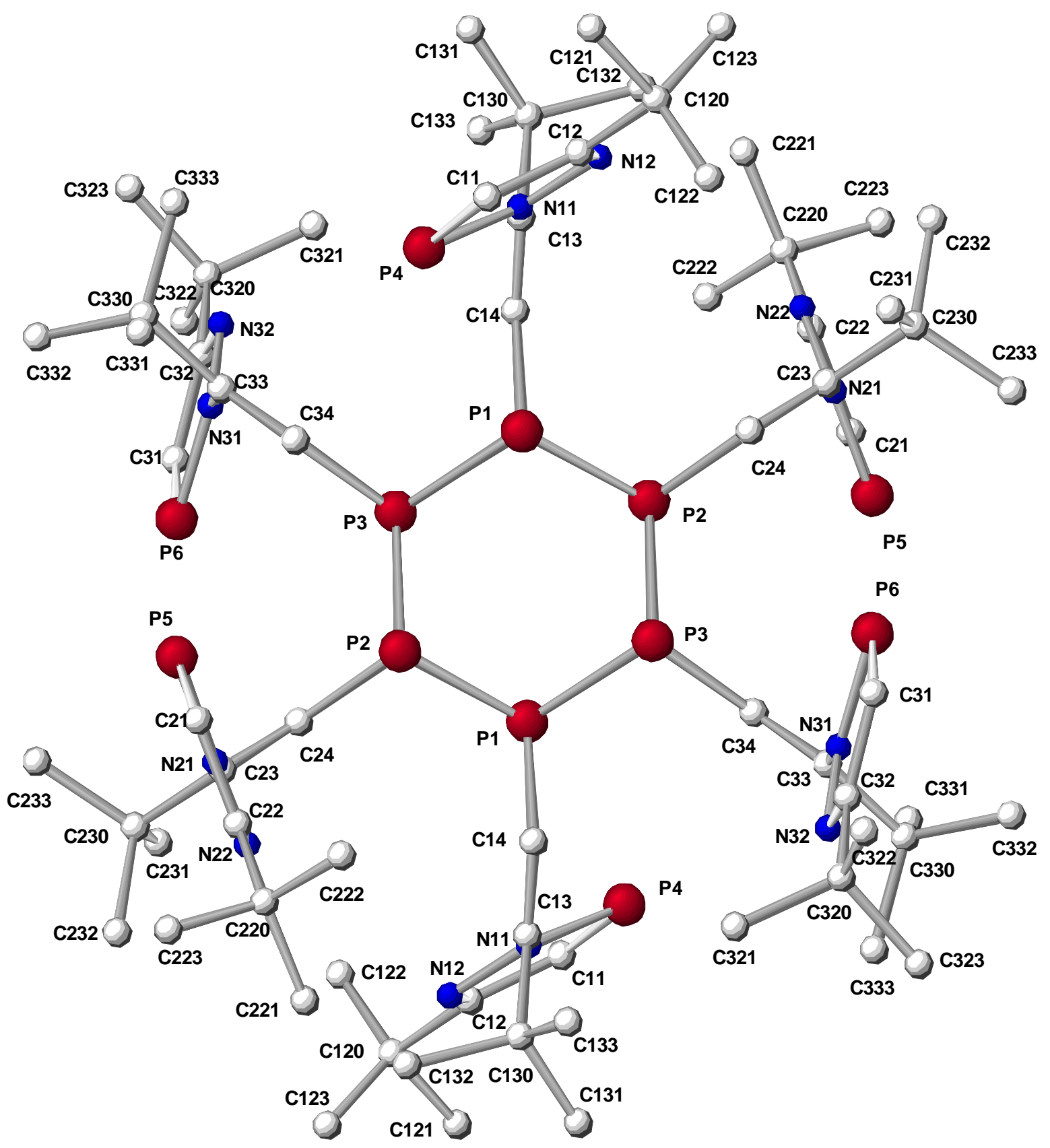

Abbildung 27: Struktur von Verbindung $\underline{25}$ (ohne Wasserstoffatome)

Die Moleküle kristallisieren monoklin mit der Raumgruppe C2/c. In den Abbildungen 28 und 29 sind Ausschnitte der Gesamtstruktur zur besseren Übersicht wiedergegeben.

Der zentrale sechsgliedrige Phosphorring zeigt eine Sesselkonformation. Die Substituenten sind jeweils äquatorial ausgerichtet. 


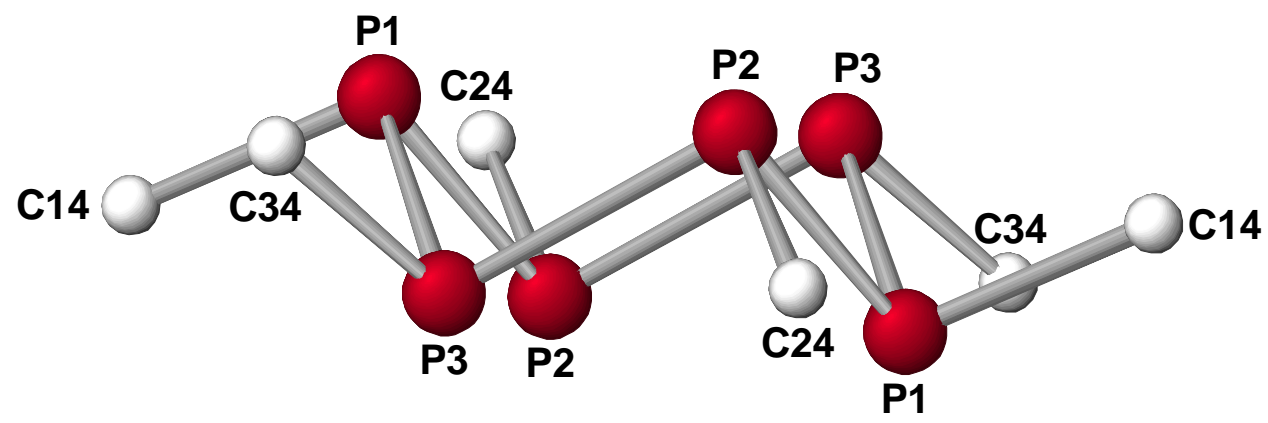

Abbildung 28: $\mathrm{P}_{6}$-Ring von Verbindung $\underline{25}$ mit dem Methylenkohlenstoffatom des Substituenten

\begin{tabular}{|cc||cc|}
$P(1)-P(2)$ & 224,6 & 223,8 \\
$P(2)-P(3)$ & 223,6 \\
$P(3)-P(1)$ & 182,2 & $P(1)-P(2)-P(3)$ & 91,3 \\
$P(1)-C(14)$ & $P(2)-P(3)-P(1)$ & 94,0 \\
$P(3)-P(1)-P(2)$ & 95,3 \\
& $\Sigma$ Innenwinkel & 561,2
\end{tabular}

Tabelle 8: Ausgewählte Bindungslängen $(\mathrm{pm})$ und -winkel $\left({ }^{\circ}\right)$ des $\mathrm{P}_{6}$-Rings von Verbindung $\underline{\mathbf{2 5}}$

Je vier der Phosphoratome spannen eine Ebene auf, die vollkommen planar ist. Dies wird in der oberen Abbildung an der von $\mathrm{P}(3)-\mathrm{P}(2)-\mathrm{P}(3)-\mathrm{P}(2)$ gebildeten Ebene deutlich. Die Bindungswinkel entsprechen weder Tetraederwinkeln, die in Cyclohexanen angestrebt werden, noch denen eines ideal planaren Sechsecks $\left(120^{\circ}\right)$. Sie sind jedoch mit Winkeln in anderen Cyclophosphanen vergleichbar. Für (PPh) 6 konnten zwei unterschiedliche Raumgruppen ermittelt werden ${ }^{43}$. Die mittleren PPP-Winkel werden von DALY mit rund $87^{\circ}$ und $89^{\circ}$ angegeben, die damit wieder auf einen überwiegenden $p-\sigma$-Bindungsanteil des Phosphoratoms und eine nahezu ausbleibende Hybridisierung hinweisen. Die angegebenen mittleren PP- und PC-Bindungslängen stimmen mit 223,4 pm und 184,5 pm nahezu vollkommen mit denen von Verbindung $\underline{25}$ überein. Für andere arylsubstituierte Cyclohexaphosphane sind ähnliche Werte veröffentlicht ${ }^{42,44}$.

Die einzelnen Substituenten weisen eine Torsion um die $\mathrm{C}(14)-\mathrm{C}(13)-\mathrm{N}(11)-\mathrm{N}(12)-$ Kette von $124^{\circ}$ auf, während die fünfgliedrigen Ringe des Substituenten - einem aromatischen System entsprechend - planar sind. 


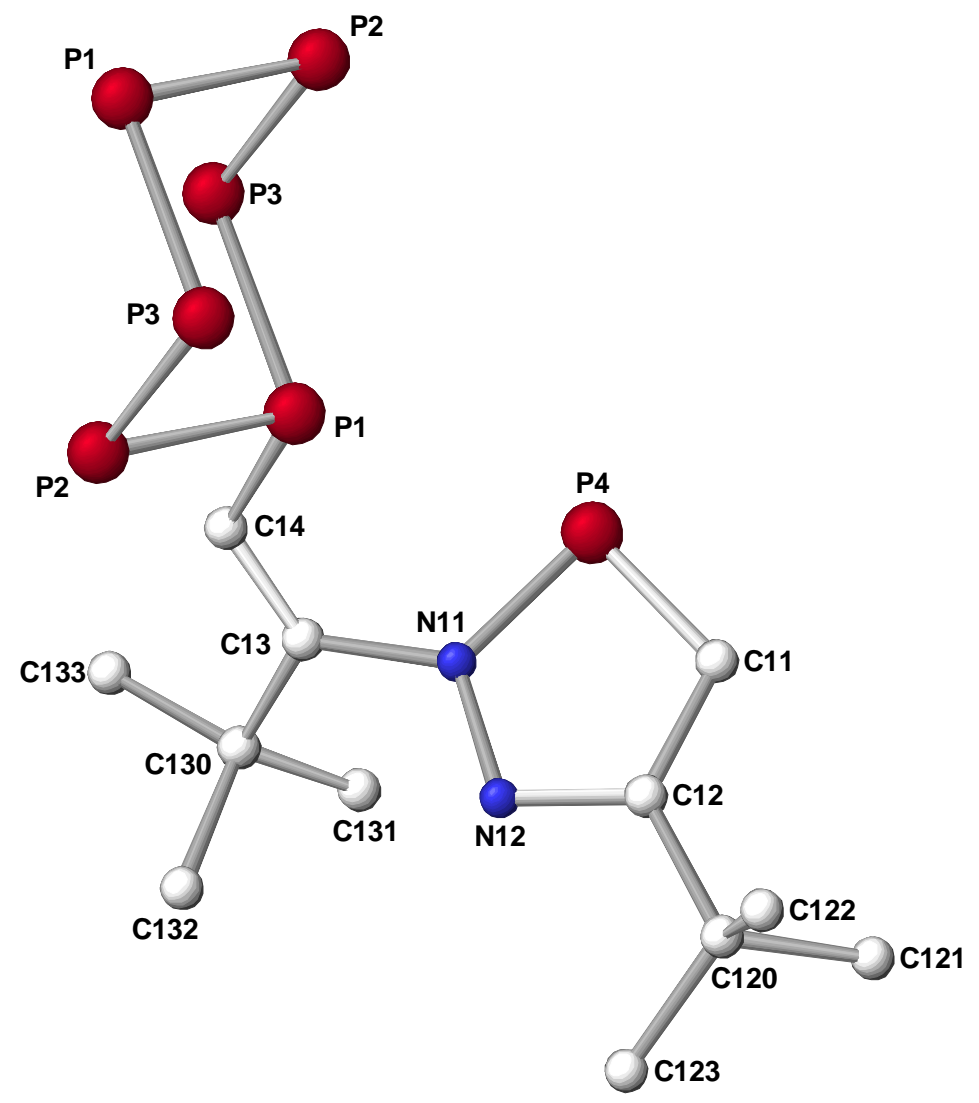

Aufsicht auf die Ringebene

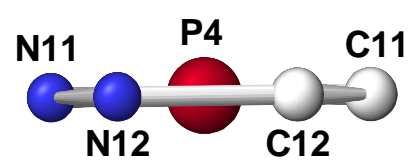

Seitenansicht der Ringebene

Abbildung 29: Ansichten des Substituenten von Verbindung $\underline{\mathbf{2 5}}$

$\begin{array}{|cc|}\mathrm{C}(11)-\mathrm{C}(12) & 139,8 \\ \mathrm{C}(12)-\mathrm{N}(12) & 133,2 \\ \mathrm{~N}(12)-\mathrm{N}(11) & 135,9 \\ \mathrm{~N}(11)-\mathrm{P}(4) & 168,8 \\ \mathrm{P}(4)-\mathrm{C}(11) & 169,7 \\ \mathrm{~N}(11)-\mathrm{C}(13) & 144,0 \\ \mathrm{C}(13)-\mathrm{C}(14) & 133,4\end{array}|\quad| \begin{array}{cc}\mathrm{C}(11)-\mathrm{C}(12)-\mathrm{N}(12) & 115,4 \\ \mathrm{C}(12)-\mathrm{N}(12)-\mathrm{N}(11) & 108,6 \\ \mathrm{~N}(12)-\mathrm{N}(11)-\mathrm{P}(4) & 116,6 \\ \mathrm{~N}(11)-\mathrm{P}(4)-\mathrm{C}(11) & 88,7 \\ \mathrm{P}(4)-\mathrm{C}(11)-\mathrm{C}(12) & 110,7 \\ \Sigma \text { Innenwinkel } & 540,0 \\ \mathrm{C} 14)-\mathrm{C}(13)-\mathrm{N}(11) & 119,6\end{array}$

Tabelle 9: Ausgewählte Bindungslängen $(\mathrm{pm})$ und -winkel $\left({ }^{\circ}\right)$ des Substituenten von Verbindung $\underline{\mathbf{2 5}}$

Im Vergleich zu den fünfgliedrigen Teilringen von Verbindung $\underline{14}$ sind alle endocyclischen Bindungen, mit Ausnahme der CC-Bindung, verkürzt. Alle Bindungen weisen damit einen partiellen Doppelbindungscharakter auf. Die Bindungslänge der $\mathrm{C}=\mathrm{C}$-Doppelbindung des Bicyclus $\underline{14}$ beträgt 135,3 pm. Die vergleichbare Bindung in dem Phospholring ( $\mathrm{C}(11)-\mathrm{C}(12)$ ist um 4,5 pm länger, aber zeigt mit 139,8 pm deutlich, dass ein aromatisches System vorliegt. Dass $\mathrm{N}(11)$ und $\mathrm{C}(13) \mathrm{sp}^{2}$ hybridisiert sind, zeigen die Winkelsummen von $359,9^{\circ}$ bzw. $360,1^{\circ}$. 
Die geringe Löslichkeit in den unterschiedlichsten Solventen $\left(C_{6} D_{6}, T H F-D_{4}, D_{2} O\right.$, $\mathrm{CDCl}_{3}, \mathrm{CS}_{2}$ ) hat bislang eine zusätzliche Charakterisierung von $\underline{\mathbf{2 5}}$ durch NMRSpektroskopie verhindert. 


\section{Zusammenfassung}

In dieser Arbeit werden neue bor- und phosphorhaltige Ringe vorgestellt, die sich bevorzugt durch thermische Dehydrohalogenierung oder über Lithiumderivate des Bis(tert.-butyl-methyl)ketazins darstellen lassen. Außerdem konnten bereits im Arbeitskreis MELLER ${ }^{6,7,8}$ dargestellte Verbindungen kristallisiert und röntgenstrukturanalytisch ausgewertet werden.

Dabei bestätigen sich die aus NMR-spektroskopischen Daten gewonnenen Strukturvorschläge für die disilylierte Ausgangsverbindung $\mathbf{I X}^{7}$ und das Azaazoniaboratacyclopenten $\mathbf{X}^{7}$ :

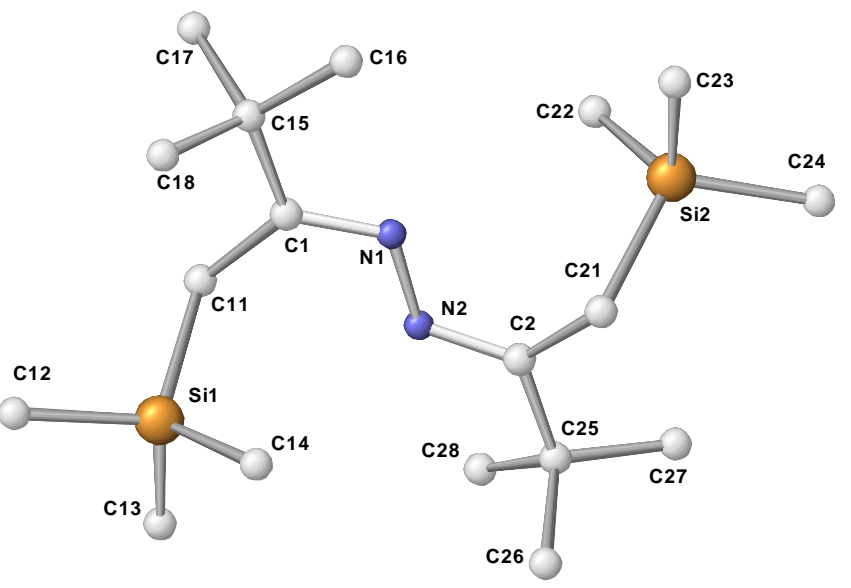

IX

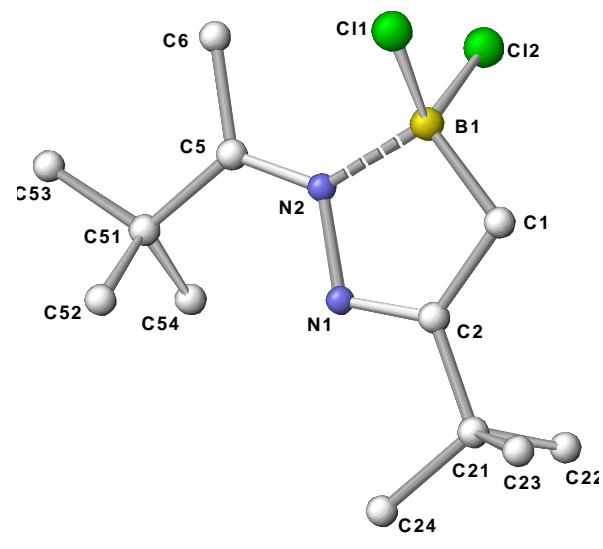

$\mathbf{X}$

Abbildung 30: Strukturen der Verbindungen IX und X (ohne Wasserstoffatome)

Die Bindungswinkel und -längen von Verbindung $\mathbf{X}$ lassen sich mit bereits bekannten bicyclischen achtgliedrigen Ringen vergleichen und stimmen mit den dort gefundenen Ergebnissen zu großen Teilen überein.

Reaktionen mit Verbindung $\mathbf{X}$ führen zu weiteren borhaltigen Ringen und ergänzen bisher gefundene Daten. Eine Substitution liefert die neue Verbindung $\underline{\mathbf{3}}$; eine Dehydrohalogenierung mit Folgereaktionen ergibt den Bicyclus $\underline{\mathbf{5}}$. 


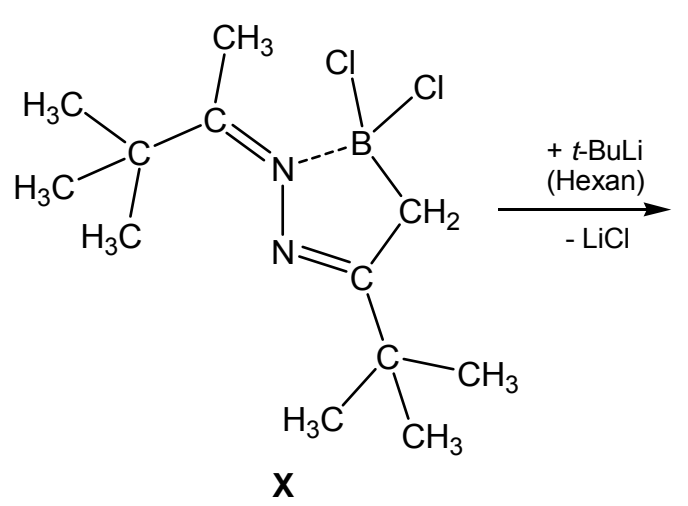<smiles></smiles>
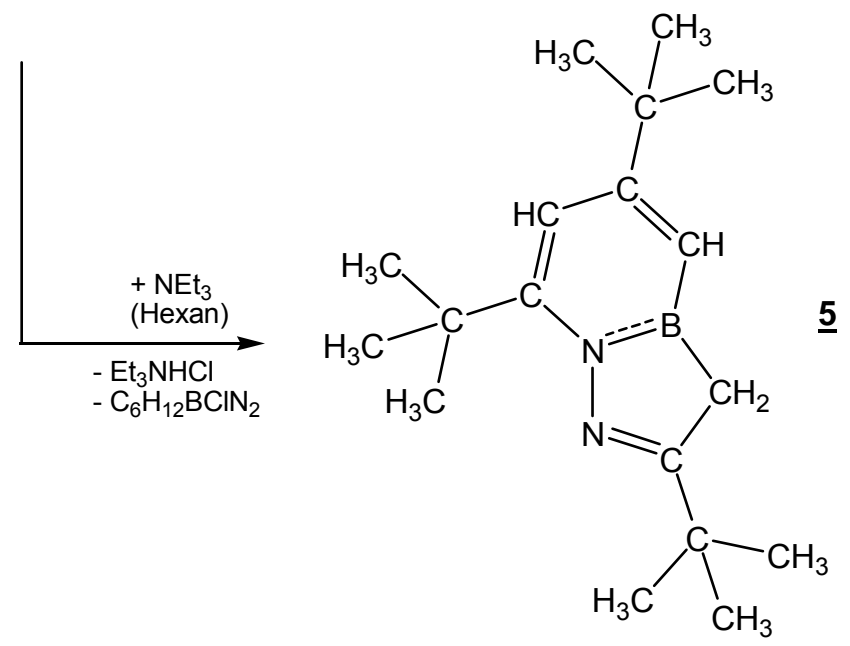

Schema 36: Weiterführende Reaktionen des borhaltigen Rings $\mathbf{X}$

Die Röntgenstrukturanalyse der Einkristalle belegt die Struktur von Verbindung $\underline{\mathbf{5}}$.

Die neuen borhaltigen Ringe $\underline{\mathbf{6}}$ und $\underline{\mathbf{9}}$ bilden sich bei der Umsetzung eines Arylbzw. Amino-substituierten Borans mit Bis(tert.-butyl-methyl)ketazin; Verbindung $\underline{\mathbf{Z}}$ kann durch Substitution des Chloratoms an $\underline{\mathbf{6}}$ erhalten werden: 
<smiles>[R]N([R])B1CC(C(C)(C)C)=NN1C(=C)C(C)(C)C</smiles>

Schema 37: Darstellung der Verbindungen $\underline{\mathbf{6}}, \underline{\mathbf{Z}}$ und $\underline{\mathbf{9}}$

Zu Verbindung $\underline{\mathbf{Z}}$ analoge diaminosubstituierte Azaazoniaboratacyclopentene sind nicht isolierbar. Einkristalle konnten sowohl von Verbindung $\underline{\mathbf{Z}}$, als auch von dem butoxisubstituierten Bicyclus $\mathbf{X V}{ }^{8}$ röntgenstrukturanalytisch untersucht werden:

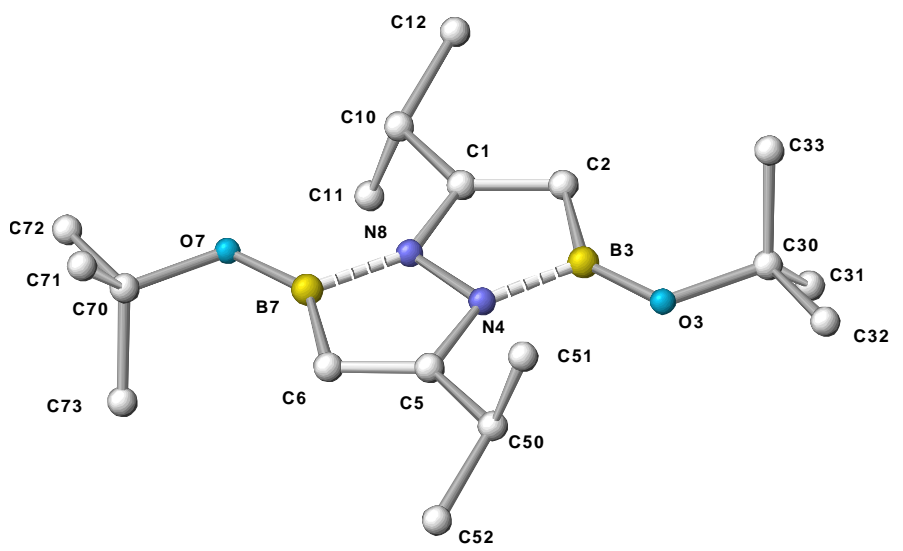

Abbildung 31: Strukturen der Verbindung $\mathbf{Z}$ und $\mathbf{X V}$ (ohne Wasserstoffatome) 
Reaktionen des Ketazins VIII mit Trichlorphosphan führen zu dem fünfgliedrigen Ring $\underline{13}$ und dem Bicyclus $\underline{14}$.<smiles>C/C(=N/N=C(\C)C(C)(C)C)C(C)(C)C</smiles><smiles>C=C(n1nc(C(C)(C)C)cp1)C(C)(C)C</smiles>

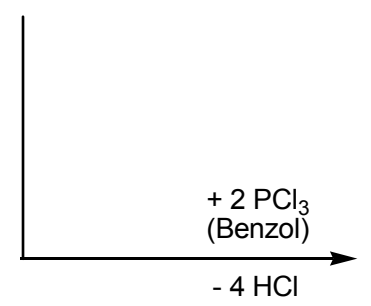<smiles>CC(C)(C)C1=CP(Cl)N2C(C(C)(C)C)=CP(Cl)N12</smiles>

Schema 38: Darstellung der Verbindungen $\underline{13}$ und $\underline{14}$

Als Nebenprodukt bei der Reaktion mit dem Dilithiumderivat von VIII entsteht das Aziniumsalz 15, das auch direkt aus Ketazin und Chlorwasserstoff dargestellt wird:<smiles>C/C(=N/N=C(\C)C(C)(C)C)C(C)(C)C</smiles>

VIII<smiles>CC(=NN([O])C(C)=C(C)C(C)(C)C)C(C)(C)C</smiles>

$\underline{15}$

Schema 39: Darstellung der Verbindung $\underline{15}$

Verbindung $\underline{14}$ und $\underline{15}$ werden als Einkristalle aus $n$-Hexan gewonnen und konnten röntgenstrukturanalytysch untersucht werden. 
Bei der Synthese phosphorhaltiger Ringe aus Halogenorganylphosphanen und dem Ketazin VIII werden die cyclischen Verbindungen $\underline{\mathbf{1 6}}$ und $\underline{19}$ isoliert:

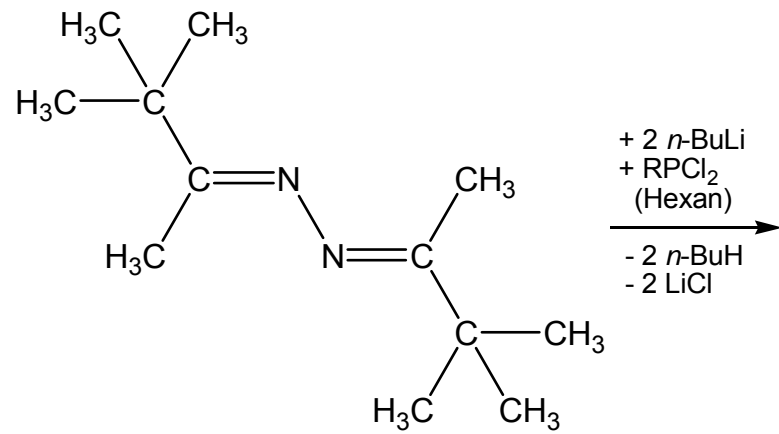

VIII<smiles>[R]P1CC(C(C)(C)C)=NN1C(=C)C(C)(C)C</smiles>

$\underline{16}, \underline{19}$

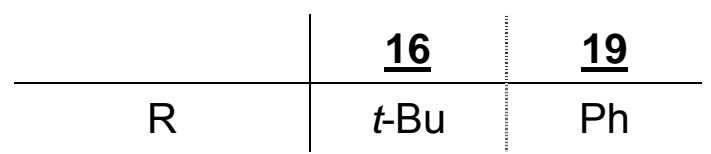

Schema 40: Darstellung der Verbindungen $\underline{16}$ und $\underline{19}$

Während Verbindung $\underline{19}$ gegenüber Feuchtigkeit und Sauerstoff über einige Zeit stabil ist, reagiert $\underline{16}$ zu dem Oxidationsprodukt $\underline{17}$ und dem Hydrolyseprodukt $\underline{18}$ :<smiles>C=C(N1N=C(C(C)(C)C)CP1C(C)(C)C)C(C)(C)C</smiles>

$\underline{16}$<smiles>C=C(N1N=C(C(C)(C)C)CP1(=O)C(C)(C)C)C(C)(C)C</smiles>

17

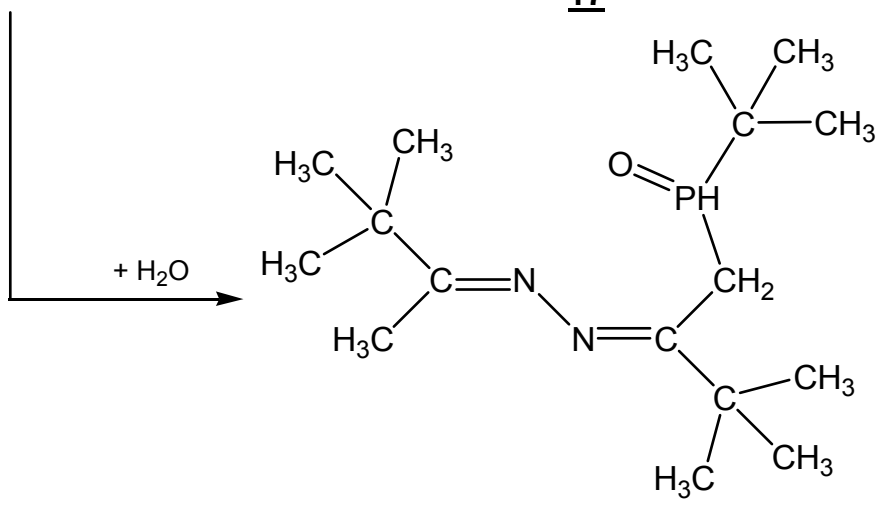

18

Schema 41: Oxidation und Hydrolyse der Verbindung $\underline{16}$ 
Die Molekülstruktur der Verbindung $\underline{17}$ wird durch eine Röntgenstrukturanalyse bestätigt.

Die Umsetzung des monolithiierten disilylierten Ketazins IX mit einem Aminodifluorphosphan zeigt eine Fluortrimethylsilan-Abspaltung und die Bildung des Diazaphosphacyclopentens 21:

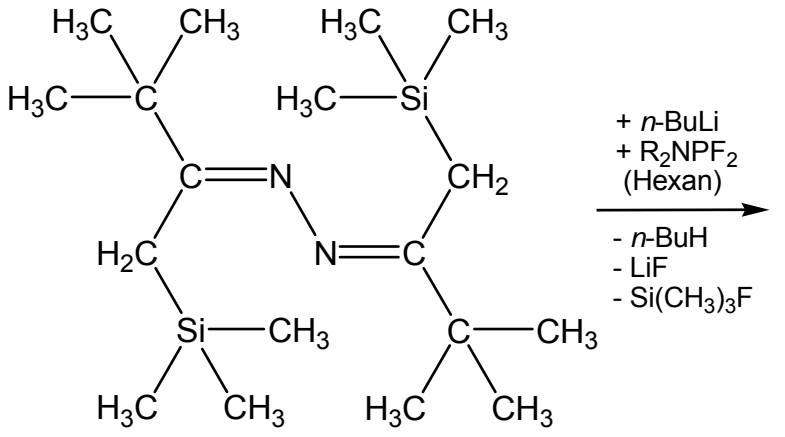

IX

$\mathrm{R}=\mathrm{CH}\left(\mathrm{CH}_{3}\right)_{2}$<smiles>[R]N([R])P1CC(C(C)(C)C)=NN1/C(=C/[Si](C)(C)C)C(C)(C)C</smiles>

$\underline{21}$

Schema 42: Darstellung von Verbindung $\underline{21}$

Die Röntgenstrukturanalyse der Verbindung $\underline{21}$ bestätigt die Struktur.

Substitutionen des Chloratoms von Verbindung 14 mit Lithiumorganylen zeigen unterschiedliche Ergebnisse. Während die sterisch anspruchsvolleren Organylreste nicht zuweisbare Substitutionsprodukte liefern, spaltet Methyllithium den Bicyclus $\underline{14}$ zu dem fünfgliedrigen Ring $\underline{\mathbf{2 3}}$. $\underline{\mathbf{2 3}}$ entsteht auch bei der Umsetzung des Hydrazons XVI mit Trichlorphosphan:

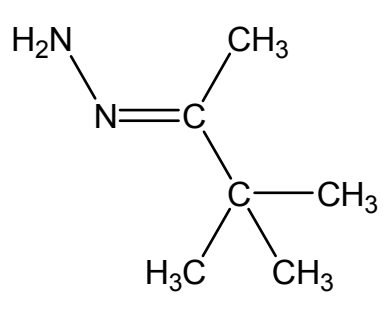

$\mathrm{XVI}$

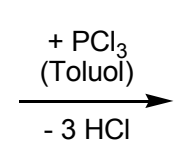

Schema 43: Darstellung von Verbindung $\underline{23}$

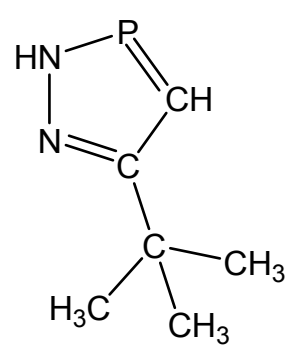

$\underline{23}$ 
Die Reduktion des Bicyclus 14 mit Kalium-Natrium-Legierung liefert als einziges isolierbares Produkt das Cyclohexaphosphan $\underline{\mathbf{2 5}}$ in geringer Ausbeute:<smiles>CC(C)(C)C1=CP(Cl)N2C(C(C)(C)C)=CP(Cl)N12</smiles>

14

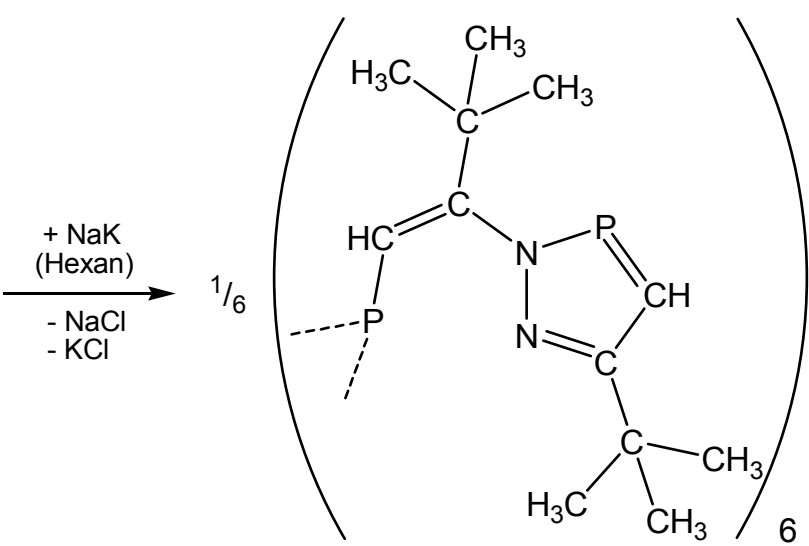

$\underline{25}$

Schema 44: Darstellung von Verbindung $\underline{\mathbf{2 5}}$

Einkristalle des Cyclohexaphosphans $\underline{25}$ bestätigen die Struktur:
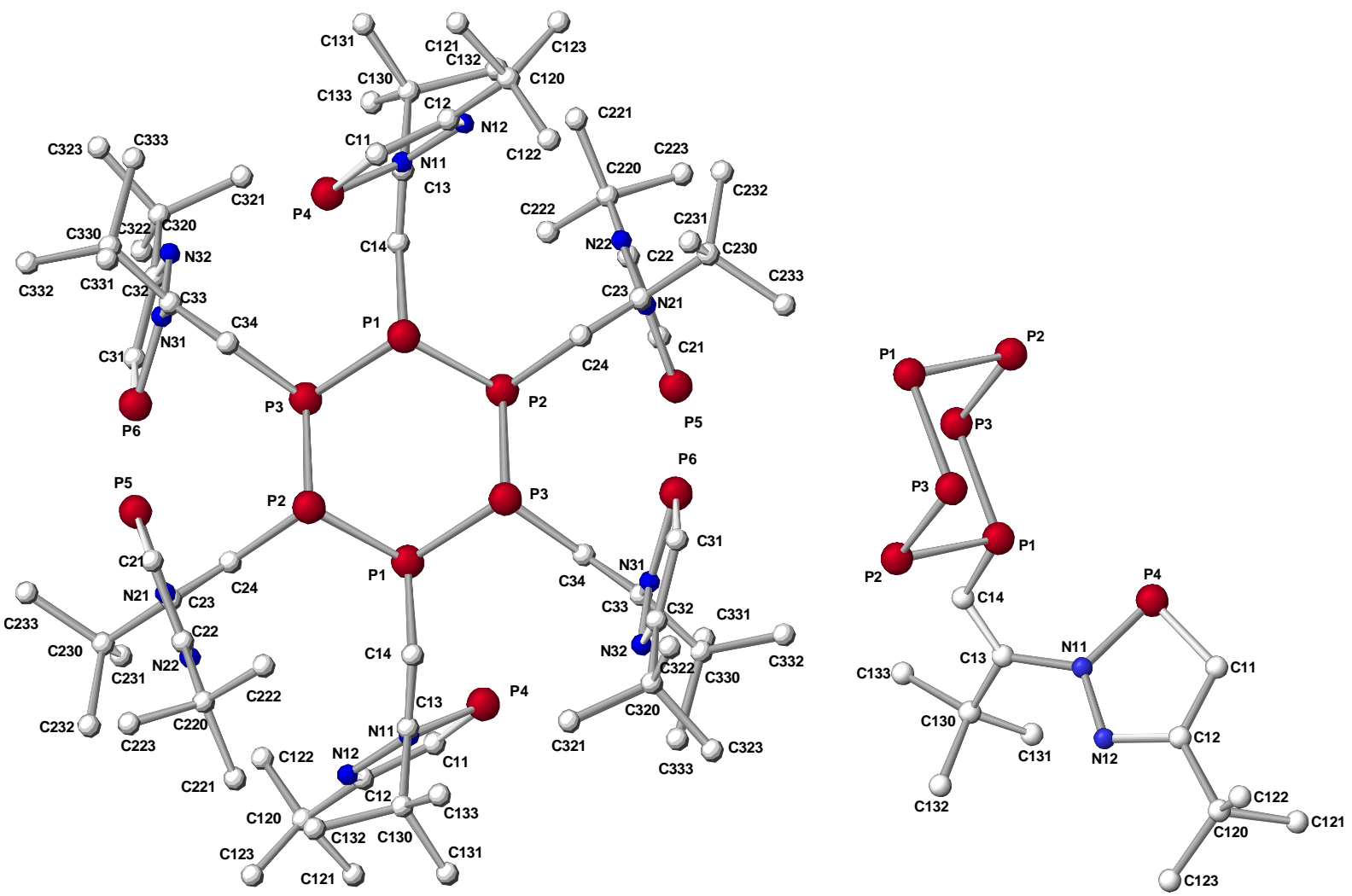

Abbildung 32: Struktur von Verbindung $\underline{25}$ 


\section{Ausblick}

Die Strukturen von IX und $\mathbf{X}$ legen nahe, dass in den vorausgehenden Lithiumderivaten des Ketazins ein deutlicher Bindungsanteil des Lithiumatoms zu den $\alpha$ Kohlenstoffatomen vorliegt. In Reaktionen mit Lithiumderivaten der nah verwandten Hydrazone werden Aminoborane gebildet. Dort ist das Metall an dem deprotonierten amidischen Stickstoffatom lokalisiert. Eine vergleichbare räumliche Anordnung lässt sich bei den Ketazinen durch die Imin-Enamin-Tautomerie formulieren. Diese erklärt aber nicht die entstehende BC-Bindung und die anschließende Stabilisierung des Moleküls durch eine intramolekulare $\mathrm{N} \rightarrow \mathrm{B}$-Adduktbildung. Röntgenstrukturanalysen der Lithiumsalze sind zur endgültigen Aufklärung nötig.

Weitere Umsetzungen Alkyl-, Aryl-, Alkoxi- und Amino-substituierter Halogenphosphane mit dem Bis(tert.-butyl-methyl)ketazin VIII sollten durchgeführt werden. Durch Variation der Ketazin-Substituenten kann der sterische Einfluss auf die Produktbildung untersucht werden. In der Bor-Azin-Chemie wurde damit bereits begonnen.

Die Synthese acyclischer Phosphor-Azin-Verbindungen ist bislang vernachlässigt worden. Hierüber sind vielleicht Vorstufen zu Ringsystemen darstellbar, durch deren Struktur mechanistische Erkenntnisse gewonnen werden können.

Weiterführende Reaktionen an den fünf- und achtgliedrigen Ringen stehen noch aus. Die versuchten Substitutionsreaktionen eines Halogens am bicyclischen Ring $\underline{14}$ sind beispielsweise noch nicht aufgeklärt. Substitutionen ohne Salzeliminierung könnten hier zum Erfolg führen.

Auch besitzen die Diazaphosphacyclopentene am Ring ein acides Proton, das zu Ringkopplungen oder Substitutionen genutzt werden kann.

Übergangsmetallkomplexe von Phospholen sind bekannt. Untersuchungen über die Bindungsmöglichkeiten neu synthetisierter Ringe sind vielversprechend. Koordinationen können über das aromatische System, einzelne Ringatome oder über die exocyclische Doppelbindung erfolgen. 
Durch Wahl weiterer Hauptgruppenelementverbindungen (z.B. Germane, Silane, Stannane oder Alane) würde eine bessere Vergleichbarkeit der Azinchemie erreicht. Durch eine anschließende Kopplung unterschiedlich heteroatomhaltiger Ringe wären interessante Moleküle darstellbar. 


\section{6 Übersicht über neue Verbindungen}

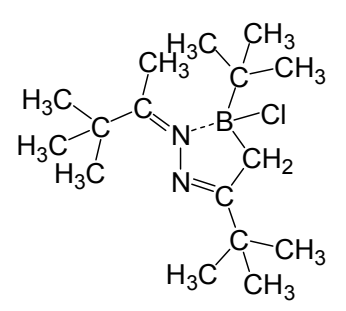

$\underline{3}$

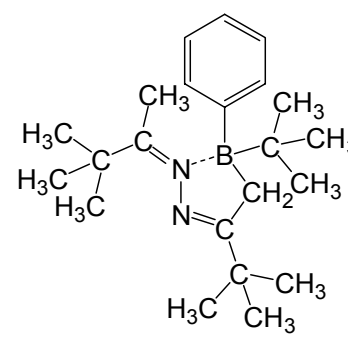

$\underline{7}$

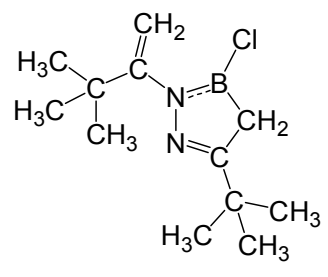

4

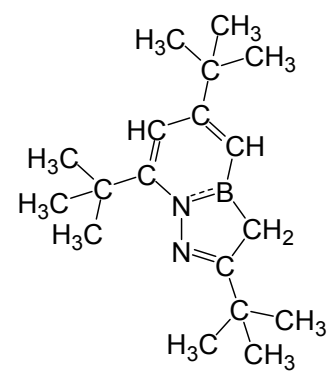

$\underline{5}$

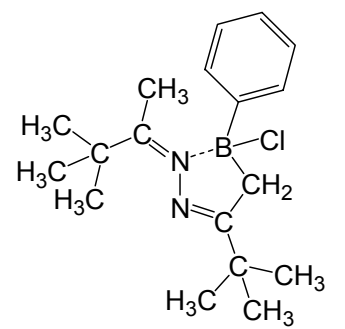

$\underline{6}$

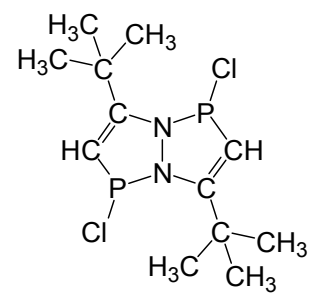

$\underline{14}$<smiles>CC(=N[NH+](Cl)C(C)C(C)(C)C)C(C)(C)C</smiles>

$\underline{15}$

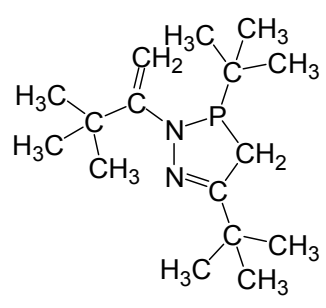

$\underline{16}$

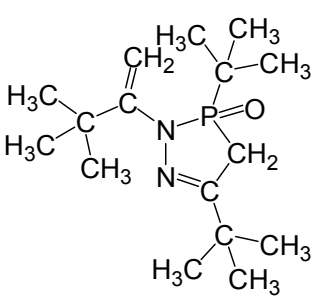

$\underline{17}$

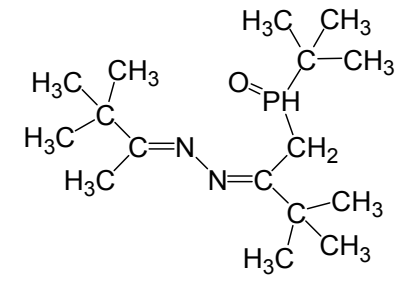

$\underline{18}$

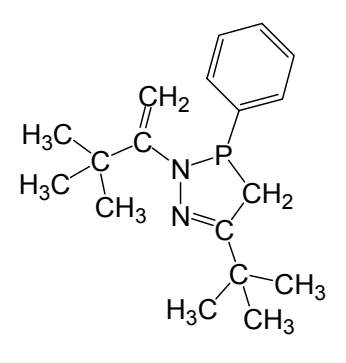

$\underline{19}$

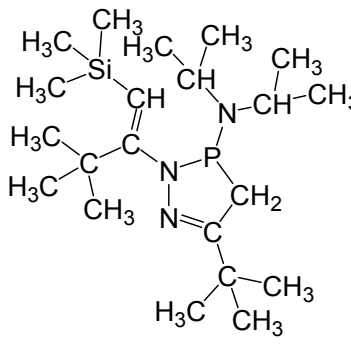

$\underline{21}$

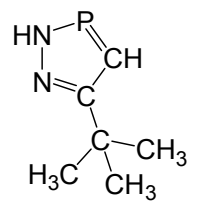

$\underline{23}$<smiles>[R]P1P([R])P([R])P([R])P([R])P1[R]</smiles><smiles>[R]=C/C=C(/n1nccp1)C(C)(C)C</smiles>

$\underline{25}$

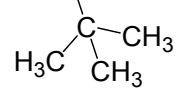




\section{Präparativer Teil}

\subsection{Allgemeine Arbeitsmethoden}

Alle Reaktionen wurden in trockener Stickstoff- oder Argonatmosphäre durchgeführt. Eine Ausnahme bildet die Synthese des Ketazins, bei dessen Darstellung durch Kondensation Wasser entsteht. Die verwendeten Lösungsmittel wurden nach gängigen Methoden getrocknet, gereinigt und aufbewahrt ${ }^{58}$.

Das als Ausgangssubstanz verwendete Keton, das Hydrazin-Hydrat, das Trichlorboran und -phosphan sind handelsüblich und wurden unverändert eingesetzt. Aryl-, Alkyl- und Aminoborane und -phosphane wurden in Anlehnung an Literaturvorschriften dargestellt ${ }^{59}$. Die eingesetzten Basen $n$-Butyllithium (23\%ig in $n$-Hexan), tert.-Butyllithium (15\%ig in $n$-Pentan), Methyllithium (5\%ig in Diethylether) und Phenyllithium (2,0 molar in Cyclohexan/Diethylether) sind ebenfalls käuflich zu erwerben. Das verwendete Ketazin, sowie seine Lithiumsalz- und Silylderivate, sind in Anlehnung an $\mathrm{GROH}^{6,7}$ dargestellt. Auch die Verbindung $\underline{\mathbf{3}}, \mathbf{4}, \underline{\mathbf{5}}, \underline{\mathbf{6}}, \underline{\mathbf{7}}, \underline{\mathbf{9}}, \underline{\mathbf{1 9}}$, und $\underline{\mathbf{2 1}}$ wurden ähnlich der dort beschriebenen Methoden synthetisiert, während $X, \underline{13}, \underline{14}$, $\underline{\mathbf{1 6}}, \underline{\mathbf{2 3}}$ und $\underline{\mathbf{2 5}}$ analog zu SCHMIDPETER ${ }^{25,24,26,27}$ erhalten wurden.

Der Verlauf der Reaktionen wurde mit Hilfe von ${ }^{1} \mathrm{H}-,{ }^{11} \mathrm{~B}-,{ }^{31} \mathrm{P}$ - und teilweise ${ }^{19} \mathrm{~F}$ Kernresonanzspektren verfolgt. Die Charakterisierung der dargestellten Verbindungen erfolgte ebenfalls durch kernresonanzspektroskopische Messungen und mittels Massenspektrometrie durch Elektronenstoßionisation.

Die Erstellung der Massenspektren erfolgte an einem FINNIGAN MAT 8200- und an einem FINNIGAN MAT 95-Spektrometer mit einer Elektronenanregungsenergie von $70 \mathrm{eV}$.

Die Schmelzpunkte der dargestellten festen Substanzen wurden in abgeschmolzenen Glaskapillaren in einer Apparatur nach TOTTOLı bestimmt. 
Die Kristalldaten wurden auf einem STOE-SIEMENS-AED-Vierkreisdiffraktometer mit graphitmonochromatisierter $\mathrm{MoK}_{\alpha}$-Strahlung bei etwa $200 \mathrm{~K}$ gesammelt. Zur Strukturlösung, Verfeinerung und Dokumentation wurde das Programmsystem SHELXL-97 ${ }^{60}$ und SHELXS-97 von G.M. SHELDRICK, Universität Göttingen, verwendet. Die Methode der Verfeinerung ist die der direct methods ${ }^{61}$ mit kleinsten Quadraten an $\mathrm{F}^{2}$.

Die ${ }^{1} \mathrm{H}-,{ }^{13} \mathrm{C}-,{ }^{11} \mathrm{~B}-,{ }^{15} \mathrm{~N}-,{ }^{29} \mathrm{Si}-$ und ${ }^{31} \mathrm{P}-\mathrm{NMR}-M e s s u n g e n$ wurden an einem BRUKER AVANCE 300- und einem BRUKER AVANCE 500-Kernresonanzspektrometer vorgenommen. Dafür wurden folgende Lösungen eingesetzt:

${ }^{1} \mathrm{H}$ : $\quad 5$ \%ige Lösungen in $\mathrm{CDCl}_{3}$, interner Standard TMS

${ }^{13} \mathrm{C}$ : $\quad 30 \%$ ige Lösungen in $\mathrm{CDCl}_{3}$, interner Standard TMS, ${ }^{13} \mathrm{C}$-Verschiebungen protonenbreitbandentkoppelt, ${ }^{13} \mathrm{C}$-Editierung über DEPT

${ }^{11} \mathrm{~B}: \quad 30 \%$ ige Lösungen in $\mathrm{CDCl}_{3}$, externer Standard $\mathrm{BF}_{3} \cdot \mathrm{OEt}_{2}$

${ }^{15} \mathrm{~N}$ : $\quad 30 \%$ ige Lösungen in $\mathrm{CDCl}_{3}$, externer Standard $\mathrm{MeNO}_{2}$, teilweise protonenbreitbandentkoppelt über INEPT, teilweise unter Zusatz von $\mathrm{Cr}$ (acac) / InversGated

${ }^{29} \mathrm{Si}: \quad 30 \%$ ige Lösungen in $\mathrm{CDCl}_{3}$, interner Standard TMS, protonenbreitbandentkoppelt, über INEPT

${ }^{31} \mathrm{P}$ : $\quad 30$ \%ige Lösungen in $\mathrm{CDCl}_{3}$, externer Standard $85 \%$ ige $\mathrm{H}_{3} \mathrm{PO}_{4}$, protonenbreitbandentkoppelt

Die ${ }^{19}$ F-NMR-Messungen wurden an einem BRUKER AVANCE 200-Kernresonanzspektrometer durchgeführt. Folgende Lösungen wurden zur Messung verwendet:

${ }^{19} \mathrm{~F}: \quad 10 \%$ ige Lösungen in $\mathrm{CDCl}_{3}$, interner Standard $\mathrm{C}_{6} \mathrm{~F}_{6}$

\subsection{Behandlung und Entsorgung der Abfälle}

Die Entsorgung halogenhaltiger Lösungsmittelabfälle und der Lösungsmittelabfälle mit sonstigen Verunreinigungen erfolgte gemeinsam mit den für kernresonanzspektroskopische Untersuchungen verwendeten deuterierten Lösungsmitteln in einem Be- 
hälter für halogenierte Rückstände. Nur wenn ausgeschlossen werden konnte, dass angefallene Abfälle halogenhaltig waren, wurden sie über entsprechende Sammelbehälter der zentralen Entsorgung für halogenfreie Lösungsmittelabfälle zugeführt.

Während größere Mengen an Natriumrückständen durch Zusammenschmelzen in siedendem Toluol wieder aufbereitet werden konnten, wurden nicht mehr verwendbare Rückstände mit Ethanol und anschließend mit Wasser vernichtet. Danach wur-

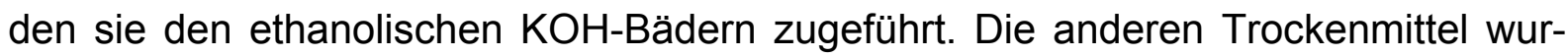
den entweder direkt entsorgt $\left(\mathrm{CaCl}_{2}\right)$ oder zunächst vorsichtig in Wasser hydrolysiert und neutralisiert $\left(\mathrm{P}_{4} \mathrm{O}_{10}\right)$.

Das zum Vorreinigen und Nachspülen der Glasgeräte verwendete Aceton wurde in einem dafür vorgesehenen Behälter gesammelt, durch Destillation gereinigt und danach erneut eingesetzt.

Alle Glasgeräte wurden durch Einlegen in konzentrierter ethanolischer $\mathrm{KOH}$ von Verunreinigungen und Fettrückständen gereinigt und anschließend mit salzsaurer Lösung und demineralisiertem Wasser gespült. Die entstandenen Lösungen wurden nach mehrwöchigem Gebrauch stark eingeengt und in den dafür vorgesehenen 20 LBehältern der zentralen Entsorgung zugeführt.

\subsection{Darstellungsvorschriften}

\section{Verbindung $\mathbf{X}$ :}

In $400 \mathrm{~mL}$ Benzol oder Toluol löst man 0,25 mol Bis(tert.-butyl-methyl)ketazin VIII $(49,0 \mathrm{~g})$ und gibt 1 Äq. Trichlorphosphan (34,5 g) hinzu. Die Reaktionsmischung wird 3 d unter Rückfluss des Lösungsmittels bis zum Sieden erhitzt und gelegentlich mit trockenem Stickstoffgas durchspült. Das Lösungsmittel entfernt man destillativ bei ND. Verbindung $\mathbf{X}$ wird durch eine fraktionierte Destillation des Rohprodukts bei $76{ }^{\circ} \mathrm{C}$ bei HV erhalten und erstarrt in der Vorlage. Durch Umkristallisation in $n$-Hexan erhält man farblose bis leicht gelbe Einkristalle. 


\section{Verbindung $\underline{3}$ :}

$0,05 \mathrm{~mol}$ von Verbindung $\mathbf{X}(14,0 \mathrm{~g})$ werden in $100 \mathrm{~mL}$ Hexan gelöst und auf $0{ }^{\circ} \mathrm{C}$ gekühlt. Danach gibt man 0,05 mol tert.-Butyllithium (15\%ig in Pentan, $170 \mathrm{~mL}$ ) hinzu. Man erwärmt die Reaktionsmischung im Laufe von $3 \mathrm{~h}$ auf RT und rührt anschlieBend weitere $2 \mathrm{~h}$ ohne Erhitzen. Bei HV entfernt man das Lösungsmittel. Das Produktgemisch wird bei HV fraktioniert destilliert und Verbindung $\underline{\mathbf{3}}$ fällt bei $82^{\circ} \mathrm{C}$ bei $\mathrm{HV}$ als viskose gelbe Flüssigkeit an, die in der Vorlage erstarrt.

\section{Verbindungen 4 und $\underline{\mathbf{5}}$ :}

Zu 0,1 mol in $250 \mathrm{~mL} n$-Hexan gelöster Verbindung $X$ werden 2 bzw. 4 Äq. Triethylamin $(18,0 \mathrm{~g}$ bzw. $36,0 \mathrm{~g})$ unter Rühren bei RT über $2 \mathrm{~h}$ gegeben. Die entstehende gelbe Aufschlämmung wird filtriert und der Rückstand mit $n$-Hexan gespült. Der Filterkuchen wird verworfen und das Lösungsmittel von dem Filtrat bei HV entfernt. Das Rohprodukt wird durch fraktionierte Sublimation gereinigt und Verbindung 4 bzw. $\underline{\mathbf{5}}$ je als gelber Feststoff gewonnen. Dabei erhält man Verbindung 4 bei $\sim 80^{\circ} \mathrm{C}$ und $\underline{\mathbf{5}}$ bei $126{ }^{\circ} \mathrm{C}$ bei je $0,1 \mathrm{mbar}$.

\section{Verbindung $\underline{6}$ :}

In $200 \mathrm{~mL} n$-Hexan löst man 0,25 mol Ketazin VIII und tropft unter Rühren 1 Äq. $n$ Butyllithium (23\%ig in $n$-Hexan, $106 \mathrm{~mL}$ ) zu. Nach $3 \mathrm{~h}$ ist die Metallierung abgeschlossen und man kühlt die Aufschlämmung bis auf $-60^{\circ} \mathrm{C}$. Das Lithiumsalz wird anschließend langsam zu $50 \mathrm{~mL} n$-Hexan und $0,25 \mathrm{~mol}-60^{\circ} \mathrm{C}$ kaltem Phenyldichlorboran gegeben. Über 6-12 h erwärmt man das Reaktionsgemisch unter Rühren wieder auf RT und trennt das Lösungsmittel bei HV ab. Das Produktgemisch wird durch fraktionierte Sublimation gereinigt und man erhält Verbindung $\underline{\mathbf{6}}$ als gelben Feststoff bei $113^{\circ} \mathrm{C}$ bei $0,1 \mathrm{mbar}$.

\section{Verbindung 7:}

$0,05 \mathrm{~mol}$ von Verbindung $\mathbf{X}(14,0 \mathrm{~g})$ werden in $100 \mathrm{~mL}$ Hexan gelöst und auf $0{ }^{\circ} \mathrm{C}$ gekühlt. Anschließend gibt man 0,05 mol Phenyllithium (2,0 molar in Cyclohexan/Diethylether, $25 \mathrm{~mL}$ ) zu. Man erwärmt die Reaktionsmischung über einen Zeitraum von $3 \mathrm{~h}$ bis auf RT und rührt danach weitere $2 \mathrm{~h}$ ohne Erhitzen. Bei HV entfernt man das Lösungsmittel. Das Produktgemisch wird bei HV fraktioniert sublimiert, und man erhält Verbindung $\underline{7}$ bei $78^{\circ} \mathrm{C}$ bei 0,04 mbar als gelben Feststoff. 


\section{Verbindung $\underline{9}$ :}

Von Verbindung VII löst man $0,25 \mathrm{~mol}(49 \mathrm{~g})$ in $400 \mathrm{~mL} n$-Hexan und metalliert mit 2 Äq. n-Butyllithium (23\%ig in $n$-Hexan, $212 \mathrm{~mL}$ ) in der Siedehitze des Lösungsmittels. Nach abgeschlossener Lithiierung kühlt man die Salzaufschlämmung bis auf $\mathrm{RT}$, tropft sie dann langsam zu einer auf $-60^{\circ} \mathrm{C}$ gekühlten Lösung aus $150 \mathrm{~mL} n$ Hexan und $0,25 \mathrm{~mol}$ Bis(trimethylsilyl)aminofluorboran $(47,5 \mathrm{~g})$ und rührt bei $-60^{\circ} \mathrm{C}$ für $1 \mathrm{~h}$. Das Reaktionsgemisch lässt man über 6 bis $12 \mathrm{~h} \mathrm{RT} \mathrm{erreichen} \mathrm{und} \mathrm{entfernt}$ anschließend das Lösungsmittel bei HV. Durch fraktionierte Sublimation bei ÖV und $82^{\circ} \mathrm{C}$ gewinnt man Verbindung $\underline{9}$ als gelben Feststoff.

\section{Verbindung $\underline{13}$ und $\underline{14}$ :}

Zu einer Lösung von 0,5 mol Ketazin VIII $(98,0 \mathrm{~g})$ in $500 \mathrm{~mL}$ Benzol oder Toluol gibt man bei RT 1,2 bzw. 2,5 Äq. Trichlorphosphan (165 g bzw. 344,0 g) und erhitzt $3 \mathrm{~d}$ unter Rückfluss des Lösungsmittels. Gelegentlich spült man die Reaktionslösung mit trockenem Stickstoffgas. Das Lösungsmittel wird bei ND abdestilliert und das verbleibende Produktgemisch durch fraktionierte Sublimation getrennt. Dabei entsteht ein Gemisch aus $\underline{13}$ und $\underline{14}$ im Verhältnis von 63:18 bzw. von 32: 43, wobei bei ÖV $\underline{13}$ als weißer bis hellgelber, glänzender Feststoff bei $66^{\circ} \mathrm{C}$ und $\underline{14}$ als gelber Feststoff bei $108^{\circ} \mathrm{C}$ erhalten wird. Von $\underline{14}$ erhält man durch Umkristallisation in $n$ Hexan Einkristalle, die zur Röntgenstrukturanalyse geeignet sind.

\section{Verbindung $\underline{15}$ :}

In 0,05 mol Ketazin VIII $(9,8 \mathrm{~g})$ leitet man frisch dargestelltes Chlorwasserstoffgas (aus $3 \mathrm{~g} \mathrm{NaCl}$ und $3 \mathrm{~mL}$ konzentrierter Schwefelsäure). Verbindung $\underline{\mathbf{1 5}}$ kristallisiert als weißer Feststoff aus der Reaktionsmischung. Durch Extraktion des Rohprodukts mit wenig $n$-Hexan und anschließender Umkristallisation in $n$-Hexan erhält man Verbindung $\underline{15}$ als farblose nadelförmige Einkristalle.

\section{Verbindung $\underline{16}$ und $\underline{19}$ :}

$0,25 \mathrm{~mol}$ des Ketazins VIII ( $49 \mathrm{~g}$ ) werden in $300 \mathrm{~mL}$ Toluol oder Benzol gelöst. Man gibt 0,25 mol tert.-Butyldichlorphosphan $(40 \mathrm{~g})$ bzw. 0,25 mol Phenyldichlorphosphan $(45 \mathrm{~g})$ unter Rühren hinzu und erhitzt bis zum Sieden unter Rückfluss des Lösungsmittels für $3 \mathrm{~d}$. Anschließend entfernt man durch Destillation bei ND das Lösungsmit- 
tel und reinigt das Rohprodukt durch fraktionierte Sublimation bei 0,005 mbar. Dabei erhält man $\underline{16}$ bzw. $\underline{18}$ bei $96^{\circ} \mathrm{C}$ bzw. $102^{\circ} \mathrm{C}$ als gelbe Feststoffe.

\section{Verbindung 17:}

In eine Lösung von 0,005 mol Verbindung $\underline{\mathbf{1 6}}(1,5 \mathrm{~g})$ in $30 \mathrm{~mL}$-Hexan leitet man unter Rühren trockenes Sauerstoffgas $(125 \mathrm{~mL})$ und lässt das Reaktionsgemisch für weitere $5 \mathrm{~h}$ bei RT rühren. Verbindung $\underline{17}$ erhält man durch Umkristallisation in $n$ Hexan als gelbe Kristalle, die zur Röntgenstrukturanalyse geeignet sind.

\section{Verbindung 18:}

Zu 0,01 mol der Verbindung $\underline{\mathbf{1 6}}$ (3 g) in Diethylether gibt man 1 Äq. bidestilliertes Wasser $(0,18 \mathrm{~mL})$ und rührt für $3 \mathrm{~h}$. Nach Abtrennen des Lösungsmittels bei HV erhält man durch Umkristallisation des Rohprodukts in $n$-Hexan Verbindung $\underline{\mathbf{1 5}}$ als hellgelbe Einkristalle.

\section{Verbindung 21:}

Von Verbindung IX löst man 0,25 mol (85 g) in $400 \mathrm{~mL} n$-Hexan und metalliert mit 1 Äq. n-Butyllithium (23\%ig in $n$-Hexan, $106 \mathrm{~mL}$ ) in der Siedehitze des Lösungsmittels. Nach abgeschlossener Lithiierung kühlt man die Salzaufschlämmung bis auf RT und tropft sie dann zu einer auf $-60{ }^{\circ} \mathrm{C}$ gekühlten Lösung aus $150 \mathrm{~mL} n$-Hexan und 0,25 mol Bis(isopropyl)aminofluorphosphan (42,5 g). Das Reaktionsgemisch erwärmt man unter Rühren über 6 bis $12 \mathrm{~h}$ auf RT und entfernt anschließend das Lösungsmittel bei HV. Durch fraktionierte Sublimation bei ÖV und $136{ }^{\circ} \mathrm{C}$ gewinnt man Verbindung $\underline{\mathbf{2 1}}$ als gelben Feststoff, der in $n$-Hexan Einkristalle bildet, die zur Röntgenstrukturanalyse geeignet sind.

\section{Verbindung 23:}

In $200 \mathrm{~mL}$ Benzol oder Toluol löst man 0,25 mol tert.-Butyl-methyl-hydrazon XVI $(28,5 \mathrm{~g})$ und gibt $0,25 \mathrm{~mol}$ Trichlorphosphan $(34,5 \mathrm{~g})$ hinzu. Nach $3 \mathrm{~d}$ Sieden unter Rückfluss mit gelegentlichem Spülen der Reaktionslösung mit trockenem Stickstoffgas entfernt man das Lösungsmittel durch Destillation bei ND. 
Verbindung 25:

0,055 mol einer Natrium-Kalium-Legierung werden frisch aus $0,4 \mathrm{~g}$ Natrium und 1,4 g Kalium durch Zusammenschmelzen hergestellt. Nachdem die Legierung RT erreicht hat gibt man $250 \mathrm{~mL} n$-Hexan und 0,05 mol Verbindung 14 (16 g) zu. Das Reaktionsgemisch lässt man 1 Woche unter Rückfluss des Lösungsmittels sieden und filtriert anschließend den verbleibenden Feststoff ab. Den Filterkuchen spült man mehrmals mit heißem $n$-Hexan. Nach Einengen des Filtrats auf 200 bis $250 \mathrm{~mL}$ kristallisiert Verbindung $\underline{25}$ zu kubischen Einkristallen aus der Reaktionslösung. 


\section{Charakteristische Daten}

Aus Gründen der Übersichtlichkeit sind die gesammelten Daten in zwei Kapitel getrennt. Im ersten Teil werden die allgemeinen Daten aufgeführt, der zweite Teil behandelt die Röntgenstrukturdaten der Verbindungen IX, X, XIII, 모 ㄱ, 14, 15, 17, $\underline{21}$ und $\underline{25}$.

\subsection{Namen, physikalische und chemische Eigenschaften}

Im nachfolgenden Abschnitt sind alle neu synthetisierten Verbindungen oder schon bekannte Verbindungen, bei denen neue Erkenntnisse gewonnen werden konnten, aufgeführt. Bei den bekannten Verbindungen sind die Daten der Vollständigkeit halber unter Verweis auf die entsprechende Literatur (Verbindung $\mathbf{I} \mathbf{X}^{7}, \mathbf{X}^{6}, \mathbf{X I}^{\mathbf{8}}$ ) aufgenommen.

Die Verbindungen 4, 17 und $\underline{25}$ sind nicht vollständig im Datenteil enthalten.

Bei den Verbindungen 12 und 20 gelang eine Isolierung des analytisch reinen Produkts nicht. Ihre Existenz ist jedoch massen- und/oder kernresonanzspektroskopisch nachgewiesen. 


$$
\mathrm{IX}^{7}
$$

\section{Bis(tert.-butyl-(trimethylsilyl)methyl)ketazin} oder 1,2-bis(3,3-dimethyl-1-(trimethylsilyl)butan-2-ylidene)hydrazine<smiles>CC(C)(C)/C(C[Si](C)(C)C)=N/N=C(/C[Si](C)(C)C)C(C)(C)C</smiles>

Summenformel:

$\mathrm{C}_{18} \mathrm{H}_{40} \mathrm{~N}_{2} \mathrm{Si}_{2}$

Ausbeute ${ }^{7}$ : $96 \%$

Schmelzpunkt ${ }^{7}$ :

$39^{\circ} \mathrm{C}$

Siedepunkt ${ }^{7}$ :

$75^{\circ} \mathrm{C} / 10^{-2} \mathrm{mbar}$

Molare Masse:

$340,70 \mathrm{~g} / \mathrm{mol}$

Massenspektrum (EI) ${ }^{7}: \quad 340\left[\mathrm{C}_{18} \mathrm{H}_{40} \mathrm{~N}_{2} \mathrm{Si}_{2} ; \mathrm{M}\right]^{+} 20 \%$

$325\left[\mathrm{M}-\mathrm{CH}_{3}\right]^{+} 40 \%$

$283\left[\mathrm{M}-\mathrm{C}_{4} \mathrm{H}_{9}\right]^{+} 20 \%$

$57\left[\mathrm{C}_{4} \mathrm{H}_{9}\right]^{+} 40 \%$

$\mathrm{NMR}^{7}$ :

$\begin{array}{llcc} & \delta^{1} \mathbf{H}[\mathbf{p p m}] & \mathbf{J}[\mathrm{Hz}] & \text { Integral } \\ \mathrm{Si}\left(\mathrm{C}_{3}\right)_{3} & 0,06 \mathrm{~s} . & & 18 \\ \mathrm{C}\left(\mathrm{C}_{3}\right)_{3} & 1,16 \mathrm{~s} . & & 18 \\ \mathrm{C} \underline{\mathrm{H}}_{2} & 2,02 \mathrm{~s} . & \mathbf{J}[\mathrm{Hz}] & 4 \\ & \delta^{13} \mathrm{C}[\mathrm{ppm}] & & \\ \mathrm{Si}\left(\underline{\mathrm{C}} \mathrm{H}_{3}\right)_{3} & 0,7 & & \\ \underline{\mathrm{C}} \mathrm{H}_{2} & 19,6 & \\ \mathrm{C}\left(\underline{\mathrm{C}} \mathrm{H}_{3}\right)_{3} & 28,9 & \\ \underline{\mathrm{C}}\left(\mathrm{CH}_{3}\right)_{3} & 38,5 & & \end{array}$




\section{$\delta^{29} \mathrm{Si} \mathrm{[ppm]} \mathrm{J} \mathrm{[Hz]}$}

$\underline{\text { Si }}$

$1,1 \mathrm{~s}$. 


$$
x^{6}
$$

5-tert.-Butyl-2-(3,3-dimethylbutyliden-2)-3,3-dichlor-1,2-azaazonia-3-borata-

$$
\text { cyclopent-5-en }
$$

oder 5-tert.-butyl-3,3-dichloro-3,4-dihydro-2-(3,3-dimethylbutan-2-ylidene)-2H-

$$
\text { 1,2,3-diazaborole }
$$<smiles></smiles>

Summenformel: $\quad \mathrm{C}_{12} \mathrm{H}_{23} \mathrm{BCl}_{2} \mathrm{~N}_{2}$

Ausbeute ${ }^{6}: \quad 54 \%$

Schmelzpunkt ${ }^{6}$ : $\quad 104{ }^{\circ} \mathrm{C}$

Molare Masse: $\quad 277,04 \mathrm{~g} / \mathrm{mol}$

Massenspektrum (EI) ${ }^{6}: \quad 276\left[\mathrm{C}_{12} \mathrm{H}_{23} \mathrm{~B}^{35} \mathrm{Cl}_{2} \mathrm{~N}_{2} ; \mathrm{M}\right]^{+} 5 \%$

$261\left[\mathrm{M}-\mathrm{CH}_{3}\right]^{+} 10 \%$

$241\left[\mathrm{M}-{ }^{35} \mathrm{Cl}\right]^{+} 40 \%$

$57\left[\mathrm{C}_{4} \mathrm{H}_{9}\right]^{+} 100 \%$

$\mathrm{NMR}^{6}$ :

$\begin{array}{lllc} & \delta^{1} \mathbf{H}[\mathbf{p p m}] & \mathbf{J}[\mathrm{Hz}] & \text { Integral } \\ \mathrm{C}\left(\underline{\mathrm{CH}}_{3}\right)_{3} & 1,20 \mathrm{~s} . & & 9 \\ \mathrm{C}\left(\mathrm{C}_{3}\right)_{3} & 1,39 \mathrm{~s} . & & 9 \\ \mathrm{BCH}_{2} & 2,22 \mathrm{~s} . & & 2 \\ \mathrm{C} \underline{\mathrm{H}}_{3} & 2,72 \mathrm{~s} . & \mathbf{J}[\mathrm{Hz}] & 3 \\ & \delta^{13} \mathrm{C}[\mathrm{ppm}] & & \\ \mathrm{CH}_{3} & 21,3 & & \\ \mathrm{C}\left(\underline{\mathrm{CH}}_{3}\right)_{3} & 27,8 & & \\ \mathrm{C}\left(\underline{\mathrm{C}} \mathrm{H}_{3}\right)_{3} & 28,0 & & \end{array}$




\section{$\delta^{13} \mathrm{C}[\mathrm{ppm}] \quad \mathrm{J}[\mathrm{Hz}]$}

$\mathrm{BCH}_{2} \quad 34,0 \mathrm{br}$.

$\underline{\mathrm{C}}\left(\mathrm{CH}_{3}\right)_{3} \quad 36,8$

$\underline{\mathrm{C}}\left(\mathrm{CH}_{3}\right)_{3} \quad 41,2$

$\underline{\mathrm{C}}_{\mathrm{q}}=\quad 188,6$

$\underline{\mathrm{C}}_{\mathrm{q}}=\quad 189,4$

$\delta^{11} \mathrm{~B}[\mathrm{ppm}] \quad \mathrm{J}[\mathrm{Hz}]$

B $7,4 \mathrm{~s}$. 


$$
\mathrm{XV}^{8}
$$

2,6-Di-tert.-Butoxi-4,8-diisopropyl-1,5-diaza-2,6-dibora-bicyclo[3.3.0]octa-3,7dien oder 1,5-tert.-butoxi-1,5-dihydro-3,7-diisopropyl-[1,2,3]diazaborolo[2,1- $\alpha$ $[1,2,3]$ diazaborole<smiles>CC(C)C1=CB(OC(C)(C)C)N2C(C(C)C)=CB(OC(C)(C)C)N12</smiles>

Summenformel:

$\mathrm{C}_{18} \mathrm{H}_{34} \mathrm{~B}_{2} \mathrm{~N}_{2} \mathrm{O}_{2}$

Ausbeute ${ }^{8}$ :

$90 \%$

Schmelzpunkt ${ }^{8}$ :

$284{ }^{\circ} \mathrm{C}$

Molare Masse:

$332,10 \mathrm{~g} / \mathrm{mol}$

Massenspektrum (EI) ${ }^{8}: \quad 332\left[\mathrm{C}_{18} \mathrm{H}_{34} \mathrm{~B}_{2} \mathrm{~N}_{2} \mathrm{O}_{2} ; \mathrm{M}\right]^{+} 35 \%$

$317\left[\mathrm{M}-\mathrm{CH}_{3}\right]^{+} 5 \%$

$220\left[\mathrm{M}-\mathrm{C}_{7} \mathrm{H}_{14} \mathrm{~N}\right]^{+} 100 \%$

$57\left[\mathrm{C}_{4} \mathrm{H}_{9}\right]^{+} 40 \%$

$N M R^{8}$

$\begin{array}{lllc} & \delta^{1} \mathbf{H}[\mathbf{p p m}] & \mathbf{J}[\mathbf{H z}] & \text { Integral } \\ \mathrm{CH}\left(\mathrm{CH}_{3}\right)_{2} & 1,13 \mathrm{~d} . & 3^{3} \mathrm{~J}_{\mathrm{HH}}=6,8 & 12 \\ \mathrm{OC}\left(\mathrm{CH}_{3}\right]_{3} & 1,28 \mathrm{~s} . & & 18 \\ \mathrm{C} \underline{\mathrm{H}}\left(\mathrm{CH}_{3}\right)_{2} & 2,95 \mathrm{sept} & { }^{3} \mathrm{~J}_{\mathrm{HH}}=6,8 & 2 \\ \mathrm{BC} \underline{\mathrm{H}} & 4,41 \mathrm{~s} . & & 2\end{array}$




\section{$\delta^{13} \mathrm{C}[\mathrm{ppm}] \quad \mathrm{J}[\mathrm{Hz}]$}

$\underline{\mathrm{C}} \mathrm{H}\left(\mathrm{CH}_{3}\right)_{2} \quad 27,7$

$\mathrm{CH}\left(\mathrm{CH}_{3}\right)_{2} \quad 21,9$

$\mathrm{OC}\left(\mathrm{CH}_{3}\right)_{3} \quad 30,5$

$\mathrm{O} \underline{\mathrm{C}}\left(\mathrm{CH}_{3}\right)_{3} \quad 73,7$

$\mathrm{B} \underline{\mathrm{CH}} \quad 91,7 \mathrm{br}$.

$\underline{\mathrm{C}}_{\mathrm{t}}=\mathrm{C} \quad 167,9$

$\delta^{11} \mathrm{~B}[\mathrm{ppm}] \quad \mathrm{J}[\mathrm{Hz}]$

B $27,5 \mathrm{~s}$. 
$\underline{3}$

3,5-Di-tert.-butyl-2-(3,3-dimethylbutyliden-2)-3-chlor-1,2-azaazonia-3-boratacyclopent-5-en

oder 3,5-di-tert.-butyl-3-chloro-3,4-dihydro-2-(3,3-dimethylbutan-2-ylidene)-2H-

1,2,3-diazaborole<smiles></smiles>

Summenformel: $\quad \mathrm{C}_{16} \mathrm{H}_{32} \mathrm{BCIN}_{2}$

Ausbeute: $\quad 24 \%$

Schmelzpunkt: $\quad 142{ }^{\circ} \mathrm{C}$

Molare Masse: $\quad 298,70 \mathrm{~g} / \mathrm{mol}$

Massenspektrum (EI): $\quad 300\left[\mathrm{C}_{16} \mathrm{H}_{32} \mathrm{~B}^{37} \mathrm{CIN}_{2}\right]^{+} 5 \%$

$298\left[\mathrm{C}_{16} \mathrm{H}_{32} \mathrm{~B}^{35} \mathrm{CIN}_{2} ; \mathrm{M}\right]^{+} 15 \%$

$283\left[\mathrm{M}-\mathrm{CH}_{3}\right]^{+} 5 \%$

$57\left[\mathrm{C}_{4} \mathrm{H}_{9}\right]^{+} 100 \%$

NMR:

$\begin{array}{lllc} & \delta^{1} \mathbf{H}[\mathbf{p p m}] & \mathbf{J}[\mathrm{Hz}] & \text { Integral } \\ \mathrm{BC}\left(\mathrm{CH}_{3}\right)_{3} & 0,70 \mathrm{~s} . & & 9 \\ \mathrm{C}\left(\mathrm{CH}_{3}\right)_{3} & 1,18 \mathrm{~s} . & & 9 \\ \mathrm{C}\left(\mathrm{C}_{3}\right)_{3} & 1,36 \mathrm{~s} . & & 9 \\ \mathrm{BC} \underline{\mathrm{H}}_{2}\left(\mathrm{H}_{\mathrm{a}}\right) & 1,77 \mathrm{~d} . & { }^{2} \mathrm{~J}_{\mathrm{HH}}=19,1 & 1 \\ \mathrm{BC} \underline{H}_{2}\left(\mathrm{H}_{\mathrm{b}}\right) & 1,95 \mathrm{~d} . & { }^{2} \mathrm{~J}_{\mathrm{HH}}=19,1 & 1 \\ \underline{\mathrm{H}}_{3} & 2,57 \mathrm{~s} . & & 3\end{array}$




\section{$\delta^{13} \mathrm{C}[\mathrm{ppm}] \quad \mathrm{J}[\mathrm{Hz}]$}

$\mathrm{CH}_{3} \quad 22,4$

$\mathrm{BC}\left(\mathrm{CH}_{3}\right)_{3} \quad 28,1$

$\mathrm{C}\left(\underline{\mathrm{CH}}_{3}\right)_{3} \quad 28,2$

$\mathrm{C}\left(\mathrm{CH}_{3}\right)_{3} \quad 28,4$

$\mathrm{B}\left(\mathrm{CH}_{3}\right)_{3} \quad 24,0$ br.

$\mathrm{BCH}_{2} \quad 33,0 \mathrm{br}$.

$\underline{\mathrm{C}}\left(\mathrm{CH}_{3}\right)_{3} \quad 36,8$

$\underline{\mathrm{C}}\left(\mathrm{CH}_{3}\right)_{3} \quad 41,3$

$\underline{\mathrm{C}}_{\mathrm{q}}=\quad 185,8$

$\underline{\mathrm{C}}_{\mathrm{q}}=\quad 189,9$

$\delta^{11} \mathrm{~B}[\mathrm{ppm}] \quad \mathrm{J}[\mathrm{Hz}]$

B $\quad 10,5 \mathrm{~s}$. 
4

5-tert.-Butyl-3-chlor-2-(3,3-dimethylbut-1-en-2-yl)-1,2-diaza-3-boracyclopent-5en

oder 5-tert.-butyl-3-chloro-3,4-dihydro-2-(3,3-dimethylbut-1-en-2-yl)-2H-1,2,3-diazaborole<smiles>C=C(N1N=C(C(C)(C)C)CB1Cl)C(C)(C)C</smiles>

Summenformel: $\quad \mathrm{C}_{12} \mathrm{H}_{22} \mathrm{BCIN}_{2}$

Ausbeute: $\quad 17 \%$

Molare Masse: $\quad 240,58 \mathrm{~g} / \mathrm{mol}$

Massenspektrum (EI): $\quad 242\left[\mathrm{C}_{12} \mathrm{H}_{22} \mathrm{~B}^{37} \mathrm{CIN}_{2}\right]^{+} 10 \%$

$240\left[\mathrm{C}_{12} \mathrm{H}_{22} \mathrm{~B}^{35} \mathrm{CIN} 2 ; \mathrm{M}\right]^{+} 40 \%$

$205\left[\mathrm{M}-\mathrm{CH}_{3}\right]^{+} 20 \%$

$195\left[\mathrm{M}-{ }^{35} \mathrm{Cl}\right]^{+} 5 \%$

$183\left[\mathrm{M}-\mathrm{C}_{4} \mathrm{H}_{9}\right]^{+} 20 \%$

$57\left[\mathrm{C}_{4} \mathrm{H}_{9}\right]^{+} 100 \%$ 
$\underline{5}$

3,6,8-Tri-tert.-butyl-4,5-diaza-1-bora-bicyclo[3.4.0]nona-1 ${ }^{5}, 3,6,8$-tetraen oder 2,6,8-tri-tert.-butyl-3H-[1,2,3]diazaborolo[2,3- $\alpha][1,2]$ azaborinine<smiles>CC(C)(C)C1=CB2N=C(C(C)(C)C)C(C(C)(C)C)=CB2C1</smiles>

Summenformel: $\quad \mathrm{C}_{18} \mathrm{H}_{31} \mathrm{BN}_{2}$

Ausbeute: $\quad 32 \%$

Siedepunkt: $\quad 126{ }^{\circ} \mathrm{C} / 0,1 \mathrm{mbar}$

Molare Masse: $\quad 286,26 \mathrm{~g} / \mathrm{mol}$

Massenspektrum (EI): $\quad 286\left[\mathrm{C}_{18} \mathrm{H}_{31} \mathrm{BN}_{2} ; \mathrm{M}\right]^{+} 100 \%$

$271\left[\mathrm{M}-\mathrm{CH}_{3}\right]^{+} 50 \%$

$229\left[\mathrm{M}-\mathrm{C}_{4} \mathrm{H}_{9}\right]^{+} 15 \%$

$57\left[\mathrm{C}_{4} \mathrm{H}_{9}\right]^{+} 35 \%$

NMR:

$\delta^{1} \mathrm{H}[\mathrm{ppm}]$

$1,28 \mathrm{~s}$.

$1,30 \mathrm{~s}$.

$1,52 \mathrm{~s}$.

$2,23 \mathrm{~s}$.

$6,41 \mathrm{~d}$.

$6,68 \mathrm{~d}$.
$\mathbf{J}[\mathrm{Hz}]$

Integral

$\mathrm{C}\left(\mathrm{C}_{3}\right)_{3}$

$\mathrm{C}\left(\mathrm{C}_{3}\right)_{3}$

$\mathrm{BC}_{2}$

$=\mathrm{C} \underline{\mathrm{H}}$

$=\underline{\mathrm{CH}}$
9

9

9

2

${ }^{4} \mathrm{~J}_{\mathrm{HH}}=2,2$

${ }^{4} \mathrm{~J}_{\mathrm{HH}}=2,2$ 


\section{$\delta^{13} \mathrm{C}[\mathrm{ppm}] \quad \mathrm{J}[\mathrm{Hz}]$}

$\mathrm{BCH}_{2} \quad 23,8 \mathrm{br}$.

$\mathrm{C}\left(\mathrm{CH}_{3}\right)_{3} \quad 29,2$

$\mathrm{C}\left(\mathrm{CH}_{3}\right)_{3} \quad 29,5$

$\mathrm{C}\left(\mathrm{CH}_{3}\right)_{3} \quad 31,1$

$\underline{\mathrm{C}}\left(\mathrm{CH}_{3}\right)_{3} \quad 35,8$

$\underline{\mathrm{C}}\left(\mathrm{CH}_{3}\right)_{3} \quad 36,6$

$\underline{\mathrm{C}}\left(\mathrm{CH}_{3}\right)_{3} \quad 36,8$

$=\underline{\mathrm{C}} \mathrm{H} \quad 104,7$

$\mathrm{B} \underline{\mathrm{CH}} \quad 114,4 \mathrm{br}$.

$=\underline{C}_{t} \quad 153,1$

$=\underline{C}_{t} \quad 166,9$

$=\underline{C}_{t} \quad 177,0$

$\delta^{11} \mathrm{~B}$ [ppm] $\quad \mathrm{J}[\mathrm{Hz}]$

B $36,1 \mathrm{br}$. 
$\underline{6}$

5-tert.-Butyl-2-(3,3-dimethylbutyliden-2)-3-chlor-3-phenyl-1,2-azaazonia-3boratacyclopent-5-en oder 5-tert.-butyl-3-chloro-3,4-dihydro-2-(3,3-dimethylbutan-2-ylidene)-3-phenyl2H-1,2,3-diazaborole<smiles></smiles>

Summenformel: $\quad \mathrm{C}_{18} \mathrm{H}_{28} \mathrm{BCIN}$

Ausbeute: $\quad 72 \%$

Siedepunkt: $\quad 113^{\circ} \mathrm{C} / 0,1 \mathrm{mbar}$

Molare Masse: $\quad 318,16 \mathrm{~g} / \mathrm{mol}$

Massenspektrum (EI): $\quad 320\left[\mathrm{C}_{18} \mathrm{H}_{28} \mathrm{~B}^{37} \mathrm{CIN}_{2}\right]^{+} 5 \%$

$318\left[\mathrm{C}_{18} \mathrm{H}_{28} \mathrm{~B}^{35} \mathrm{CIN}_{2} ; \mathrm{M}\right]^{+} 15 \%$

$303\left[\mathrm{M}-\mathrm{CH}_{3}\right]^{+} 1 \%$

$283\left[\mathrm{M}-{ }^{35} \mathrm{Cl}\right]{ }^{+} 55 \%$

$261\left[\mathrm{M}-\mathrm{C}_{4} \mathrm{H}_{9}\right]^{+} 10 \%$

$241\left[\mathrm{M}-\mathrm{C}_{6} \mathrm{H}_{5}\right]^{+} 100 \%$

$57\left[\mathrm{C}_{4} \mathrm{H}_{9}\right]^{+} 75 \%$

NMR:

$$
\delta^{1} \mathrm{H} \text { [ppm] }
$$

$\mathrm{C}\left(\mathrm{CH}_{3}\right)_{3}$

$\mathrm{C}\left(\mathrm{CH}_{3}\right)_{3}$

$\mathrm{BC}_{2}\left(\mathrm{H}_{\mathrm{a}}\right)$ 
$\delta^{1} \mathbf{H}$ [ppm]

$\mathrm{J}[\mathrm{Hz}]$

Integral

$\mathrm{CH}_{3}$

$2,13 \mathrm{~s}$.

3

$\mathrm{BC}_{2}\left(\mathrm{H}_{\mathrm{b}}\right)$

$2,22 \mathrm{~d}$.

${ }^{2} \mathrm{~J}_{\mathrm{HH}}=19,9$

1

$\mathrm{B}\left(\mathrm{C}_{6} \underline{\mathrm{H}}_{5}\right)$

$7,1-7,4 \mathrm{~m}$.

5

$\delta^{13} \mathrm{C}[\mathrm{ppm}]$

J [Hz]

$\mathrm{C}_{3} \quad 21,5$

$\mathrm{C}\left(\mathrm{CH}_{3}\right)_{3} \quad 27,5$

$\mathrm{C}\left(\mathrm{CH}_{3}\right)_{3} \quad 28,0$

$\mathrm{BCH}_{2} \quad 34 \mathrm{br}$.

$\underline{\mathrm{C}}\left(\mathrm{CH}_{3}\right)_{3} \quad 36,4$

$\underline{\mathrm{C}}\left(\mathrm{CH}_{3}\right)_{3} \quad 40,3$

$\mathrm{B}\left(\underline{\mathrm{C}}_{6} \mathrm{H}_{5}\right)\left(\mathrm{C}_{4}\right) \quad 125,7$

$\mathrm{B}\left(\underline{\mathrm{C}}_{6} \mathrm{H}_{5}\right)\left(\mathrm{C}_{2}\right) \quad 127,3$

$\mathrm{B}\left(\underline{\mathrm{C}}_{6} \mathrm{H}_{5}\right)\left(\mathrm{C}_{3}\right) \quad 129,9$

$\mathrm{B}\left(\underline{\mathrm{C}}_{6} \mathrm{H}_{5}\right)\left(\mathrm{C}_{1}\right) \quad 148 \mathrm{br}$.

$=\underline{C}_{t} \quad 185,4$

$=C_{t} \quad 190,5$

$\delta^{11} \mathrm{~B}[\mathrm{ppm}] \quad \mathrm{J}[\mathrm{Hz}]$

B $\quad 6,2 \mathrm{~s}$. 
$\underline{7}$

3,5-Di-tert.-butyl-2-(3,3-dimethylbutyliden-2)-3-phenyl-1,2-azaazonia-3-boratacyclopent-5-en

oder 3,5-di-tert.-butyl-3,4-dihydro-2-(3,3-dimethylbutan-2-ylidene)-3-phenyl-2H1,2,3-diazaborole<smiles></smiles>

Summenformel: $\quad \mathrm{C}_{22} \mathrm{H}_{37} \mathrm{BN}_{2}$

Ausbeute: $\quad 16 \%$

Siedepunkt: $\quad 78{ }^{\circ} \mathrm{C} / 0,04 \mathrm{mbar}$

Molare Masse: $\quad 340,25 \mathrm{~g} / \mathrm{mol}$

Massenspektrum (EI): $\quad 325\left[\mathrm{C}_{21} \mathrm{H}_{34} \mathrm{BN}_{2} ; \mathrm{M}-\mathrm{CH}_{3}\right]^{+} 1 \%$

$283\left[\mathrm{M}-\mathrm{C}_{4} \mathrm{H}_{9}\right]^{+} 100 \%$

$186\left[\mathrm{HNC}\left(\mathrm{CH}_{3}\right)_{3} \mathrm{CH}_{2} \mathrm{BC}_{6} \mathrm{H}_{5}\right]^{+} 40 \%$

$57\left[\mathrm{C}_{4} \mathrm{H}_{9}\right]^{+} 80 \%$

NMR:

\begin{tabular}{|c|c|c|c|}
\hline & $\delta^{1} \mathrm{H}[\mathrm{ppm}]$ & $\mathbf{J}[\mathrm{Hz}]$ & Integral \\
\hline $\mathrm{BC}\left(\mathrm{CH}_{3}\right)_{3}$ & $0,87 \mathrm{~s}$. & & 9 \\
\hline $\mathrm{C}\left(\mathrm{CH}_{3}\right)_{3}$ & $1,25 \mathrm{~s}$. & & 9 \\
\hline $\mathrm{C}\left(\mathrm{CH}_{3}\right)_{3}$ & $1,36 \mathrm{~s}$. & & 9 \\
\hline$B C \underline{H}_{2}\left(\mathrm{H}_{\mathrm{a}}\right)$ & $1,53 \mathrm{~d}$ & ${ }^{2} \mathrm{~J}_{\mathrm{HH}}=19,4$ & 1 \\
\hline 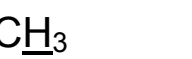 & $1,80 \mathrm{~s}$. & & 3 \\
\hline
\end{tabular}




\begin{tabular}{|c|c|c|c|}
\hline & $\delta^{1} \mathrm{H}[\mathrm{ppm}]$ & $\mathbf{J}[\mathrm{Hz}]$ & Integral \\
\hline$B C \underline{H}_{2}\left(H_{b}\right)$ & $1,94 \mathrm{~d}$ & ${ }^{2} \mathrm{~J}_{\mathrm{HH}}=19,4$ & 1 \\
\hline \multirow[t]{2}{*}{$\mathrm{B}\left(\mathrm{C}_{6} \underline{\mathrm{H}}_{5}\right)$} & $7,1-7,4 \mathrm{~m}$ & & 5 \\
\hline & $\delta^{13} \mathrm{C}[\mathrm{ppm}]$ & $\mathbf{J}[\mathrm{Hz}]$ & \\
\hline $\mathrm{B} \underline{\mathrm{C}}\left(\mathrm{CH}_{3}\right)_{3}$ & 22,8 br. & & \\
\hline$\underline{\mathrm{CH}}_{3}$ & 23,8 & & \\
\hline $\mathrm{C}\left(\underline{\mathrm{CH}}_{3}\right)_{3}$ & 28,1 & & \\
\hline $\mathrm{C}\left(\underline{\mathrm{CH}}_{3}\right)_{3}$ & 28,7 & & \\
\hline $\mathrm{BC}\left(\underline{\mathrm{CH}}_{3}\right)_{3}$ & 30,4 & & \\
\hline $\mathrm{BC}^{\mathrm{C}} \mathrm{H}_{2}$ & $33 \mathrm{br}$. & & \\
\hline$\underline{\mathrm{C}}\left(\mathrm{CH}_{3}\right)_{3}$ & 36,9 & & \\
\hline$\underline{\mathrm{C}}\left(\mathrm{CH}_{3}\right)_{3}$ & 40,5 & & \\
\hline $\mathrm{B}\left(\underline{\mathrm{C}}_{6} \mathrm{H}_{5}\right)\left(\mathrm{C}_{4}\right)$ & 124,2 & & \\
\hline $\mathrm{B}\left(\underline{\mathrm{C}}_{6} \mathrm{H}_{5}\right)\left(\mathrm{C}_{2}\right)$ & 127,3 & & \\
\hline $\mathrm{B}\left(\underline{\mathrm{C}}_{6} \mathrm{H}_{5}\right)\left(\mathrm{C}_{3}\right)$ & 132,1 & & \\
\hline $\mathrm{B}\left(\underline{\mathrm{C}}_{6} \mathrm{H}_{5}\right)\left(\mathrm{C}_{1}\right)$ & $154,5 \mathrm{br}$. & & \\
\hline$=C_{t}$ & 181,1 & & \\
\hline \multirow[t]{2}{*}{$=C_{t}$} & 191,6 & & \\
\hline & $\delta^{11} \mathrm{~B}[\mathrm{ppm}]$ & $\mathbf{J}[\mathrm{Hz}]$ & \\
\hline$\underline{B}$ & $3,7 \mathrm{~s}$. & & \\
\hline
\end{tabular}


$\underline{9}$

3-Bis(trimethylsilyl)amino-5-tert.-butyl-2-(3,3-dimethylbutyliden-2)-1,2-diaza-3boracyclopent-5-en

oder 3-bis(trimethylsilyl)amino-5-tert.-butyl-3,4-dihydro-2-(3,3-dimethylbut-1-en2-yl)-2H-1,2,3-diazaborole<smiles>C=C(N1N=C(C(C)(C)C)CB1N([Si](C)(C)C)[Si](C)(C)C)C(C)(C)C</smiles>

Summenformel: $\quad \mathrm{C}_{18} \mathrm{H}_{40} \mathrm{BN}_{3} \mathrm{Si}_{2}$

Ausbeute: $66 \%$

Sublimationspunkt: $82{ }^{\circ} \mathrm{C} / 0,01 \mathrm{mbar}$

Molare Masse: $365,51 \mathrm{~g} / \mathrm{mol}$

Massenspektrum (EI): $\quad 365\left[\mathrm{C}_{18} \mathrm{H}_{40} \mathrm{BN}_{3} \mathrm{Si}_{2} ; \mathrm{M}\right]^{+} 10 \%$ $350\left[\mathrm{M}-\mathrm{CH}_{3}\right]^{+} 10 \%$ $308\left[\mathrm{M}-\mathrm{C}_{4} \mathrm{H}_{9}\right]^{+} 100 \%$

NMR:

\begin{tabular}{|c|c|c|c|}
\hline & $\delta^{1} \mathrm{H}[\mathrm{ppm}]$ & $\mathbf{J}[\mathrm{Hz}]$ & Integral \\
\hline $\mathrm{Si}\left(\mathrm{C}_{3}\right)_{3}$ & $0,11 \mathrm{~s}$. & & 18 \\
\hline $\mathrm{C}\left(\mathrm{CH}_{3}\right)_{3}$ & $1,14 \mathrm{~s}$. & & 9 \\
\hline $\mathrm{C}\left(\mathrm{CH}_{3}\right)_{3}$ & $1,24 \mathrm{~s}$. & & 9 \\
\hline $\mathrm{BCH}_{2}$ & $1,77 \mathrm{~s}$ & & 2 \\
\hline$=\underline{C H}_{2}\left(\mathrm{H}_{\mathrm{a}}\right)$ & $4,77 \mathrm{~s}$ & & 1 \\
\hline$=\mathrm{C}_{2}\left(\mathrm{H}_{\mathrm{b}}\right)$ & 4,92 s. & & 1 \\
\hline
\end{tabular}




\section{$\delta^{13} \mathrm{C}[\mathrm{ppm}] \quad \mathrm{J}[\mathrm{Hz}]$}

$\mathrm{Si}\left(\mathrm{CH}_{3}\right)_{3} \quad 3,4$

$\mathrm{BCH}_{2} \quad 27 \mathrm{br}$.

$\mathrm{C}\left(\mathrm{C}_{3}\right)_{3} \quad 28,8$

$\mathrm{C}\left(\mathrm{CH}_{3}\right)_{3} \quad 30,4$

$\underline{\mathrm{C}}\left(\mathrm{CH}_{3}\right)_{3} \quad 35,2$

$\underline{\mathrm{C}}\left(\mathrm{CH}_{3}\right)_{3} \quad 36,8$

$=\underline{\mathrm{C}} \mathrm{H}_{2}$

$\underline{\mathrm{C}}_{\mathrm{t}}=\mathrm{C} \quad 156,2$

$\underline{\mathrm{C}}_{\mathrm{t}}=\mathrm{N} \quad 167,2$

$\delta^{29} \mathrm{Si}[\mathrm{ppm}] \quad \mathrm{J}[\mathrm{Hz}]$

Si $\quad 0,7 \mathrm{~d}$.

$\delta^{11} \mathrm{~B}$ [ppm] $\quad \mathrm{J}[\mathrm{Hz}]$

B $\quad 41,5 \mathrm{~s}$. 
13

5-tert.-Butyl-2-(3,3-dimethylbut-1-en-2-yl)-1,2-diaza-3-phosphacyclopenta-3,5dien oder 5-tert.-butyl-2-(3,3-dimethylbut-1-en-2-yl)-2H-1,2,3-diazaphosphole<smiles>C=C(n1nc(C(C)(C)C)cp1)C(C)(C)C</smiles>

Summenformel: $\quad \mathrm{C}_{12} \mathrm{H}_{21} \mathrm{~N}_{2} \mathrm{P}$

Ausbeute: $\quad 60 \%$

Sublimationspunkt: $\quad 66^{\circ} \mathrm{C} / 0,05$ mbar

Molare Masse: $\quad 224,28 \mathrm{~g} / \mathrm{mol}$

Massenspektrum (EI): $\quad 224\left[\mathrm{C}_{12} \mathrm{H}_{21} \mathrm{~N}_{2} \mathrm{P} ; \mathrm{M}\right]^{+} 80 \%$ $209\left[\mathrm{M}-\mathrm{CH}_{3}\right]^{+} 60 \%$

$167\left[\mathrm{M}-\mathrm{C}_{4} \mathrm{H}_{9}\right]^{+} 55 \%$

$57\left[\mathrm{C}_{4} \mathrm{H}_{9}\right]^{+} 100 \%$

NMR:

$\begin{array}{lllc} & \delta^{\mathbf{1}} \mathbf{H}[\mathbf{p p m}] & \mathbf{J}[\mathrm{Hz}] & \text { Integral } \\ \mathrm{C}\left(\mathrm{CH}_{3}\right)_{3} & 1,20 \mathrm{~s} . & & 9 \\ \mathrm{C}\left(\mathrm{CH}_{3}\right)_{3} & 1,34 \mathrm{~s} . & & 9 \\ =\mathrm{CH}_{2}\left(\mathrm{H}_{\mathrm{a}}\right) & 5,06 \mathrm{~d} \mathbf{v d} . & { }^{2} \mathrm{~J}_{\mathrm{HH}}=0,5 & 1 \\ & & { }^{4} \mathrm{~J}_{\mathrm{HP}}=1,2 & 1 \\ =\mathrm{CH}_{2}\left(\mathrm{H}_{\mathrm{b}}\right) & 5,18 \mathrm{~d} \mathbf{v d} & { }^{2} \mathrm{~J}_{\mathrm{HH}}=0,5 & \\ & & { }^{4} \mathrm{~J}_{\mathrm{HP}}=1,9 & 2 \\ \mathrm{PC} \underline{\mathrm{H}} & 7,56 \mathrm{~d} . & { }^{2} \mathrm{~J}_{\mathrm{HP}}=44,4 & \end{array}$




\section{$\delta^{13} \mathrm{C}[\mathrm{ppm}]$}

$\mathrm{C}\left(\underline{\mathrm{C}}_{3}\right)_{3}$

$\mathrm{C}\left(\mathrm{CH}_{3}\right)_{3}$

$\underline{\mathrm{C}}\left(\mathrm{CH}_{3}\right)_{3}$

$\underline{\mathrm{C}}\left(\mathrm{CH}_{3}\right)_{3}$

$=\mathrm{CH}_{2}$

$\mathrm{P} \underline{\mathrm{CH}}$

$=\mathrm{C}_{\mathrm{t}}$

$=\mathrm{C}_{\mathrm{t}}$

$\underline{P}=$
$29,3 \mathrm{~d}$.

$30,8 \mathrm{~d}$.

$33,9 \mathrm{~d}$.

$36,7 \mathrm{~d}$.

$107,3 \mathrm{~d}$.

$131,8 \mathrm{~d}$.

$158,6 \mathrm{~d}$.

$168,7 \mathrm{~d}$.

$\delta^{31} \mathrm{P}[\mathrm{ppm}]$

$235,0 \mathrm{~s}$.

${ }^{1}$ H-gek. d.
J [Hz]

${ }^{4} \mathrm{~J}_{\mathrm{CP}}=0,9$

${ }^{4} \mathrm{~J}_{\mathrm{CP}}=0,7$

${ }^{3} \mathrm{~J}_{\mathrm{CP}}=1,4$

${ }^{3} \mathrm{~J}_{\mathrm{CP}}=0,7$

${ }^{3} J_{C P}=9,7$

${ }^{1} \mathrm{~J}_{\mathrm{CP}}=36,1$

${ }^{2} \mathrm{~J}_{\mathrm{CP}}=8,3$

${ }^{2} \mathrm{~J}_{\mathrm{CP}}=9,0$

$\mathrm{J}[\mathrm{Hz}]$

${ }^{2} \mathrm{~J}_{\mathrm{PH}}=44,4$ 
$\underline{14}$

4,8-Di-tert.-butyl-2,6-dichlor-1,5-diaza-2,6-diphospha-bicyclo[3.3.0]octa-3,7-dien oder 3,7-di-tert-butyl-1,5-dichloro-1,5-dihydro-[1,2,3]diazaphospholo[2,1- $\alpha]$ $[1,2,3]$ diazaphosphole<smiles>CC(C)(C)C1=CP(Cl)N2C(C(C)(C)C)=CP(Cl)N12</smiles>

Summenformel: $\quad \mathrm{C}_{12} \mathrm{H}_{20} \mathrm{Cl}_{2} \mathrm{~N}_{2} \mathrm{P}_{2}$

Ausbeute: $\quad 35 \%$

Schmelzpunkt: $\quad 112{ }^{\circ} \mathrm{C}$

Molare Masse: $\quad 325,15 \mathrm{~g} / \mathrm{mol}$

Massenspektrum (EI): $\quad 326\left[\mathrm{C}_{12} \mathrm{H}_{20}{ }^{37} \mathrm{Cl}^{35} \mathrm{CIN}_{2} \mathrm{P}_{2}\right]^{+} 5 \%$ $324\left[\mathrm{C}_{12} \mathrm{H}_{20}{ }^{35} \mathrm{Cl}_{2} \mathrm{~N}_{2} \mathrm{P}_{2} ; \mathrm{M}\right]^{+} 10 \%$ $289\left[\mathrm{M}-{ }^{35} \mathrm{Cl}\right]{ }^{+} 100 \%$

NMR:

\begin{tabular}{|c|c|c|c|}
\hline & $\delta^{1} \mathrm{H}[\mathrm{ppm}]$ & $\mathrm{J}[\mathrm{Hz}]$ & Integral \\
\hline $\mathrm{C}\left(\mathrm{CH}_{3}\right)_{3}$ & $1,46 \mathrm{~s}$. & & 18 \\
\hline \multirow[t]{2}{*}{$\mathrm{PC} \underline{\mathrm{H}}$} & 5,65 m. $\underline{A A^{\prime} X X^{\prime}}$ & unaufgelöst & 2 \\
\hline & $\delta^{13} \mathrm{C}[\mathrm{ppm}]$ & $\mathrm{J}[\mathrm{Hz}]$ & \\
\hline $\mathrm{C}\left(\underline{\mathrm{CH}}_{3}\right)_{3}$ & 30,3 pst. & ${ }^{4} J_{C P}=4,5$ & \\
\hline$\underline{\mathrm{C}}\left(\mathrm{CH}_{3}\right)_{3}$ & 33,7 & & \\
\hline $\mathrm{P} \underline{\mathrm{C}} \mathrm{H}$ & 104,4 br. & & \\
\hline \multirow[t]{2}{*}{$=C_{t}$} & 161,0 & & \\
\hline & $\delta^{31} \mathrm{P}[\mathrm{ppm}]$ & $\mathrm{J}[\mathrm{Hz}]$ & \\
\hline$\underline{\mathrm{P} C l}$ & $108,6 \mathrm{~s}$. & & \\
\hline
\end{tabular}


$\underline{15}$

Bis(tert.-butyl-methyl)ketazinhydrochlorid oder 1,2-bis(3,3-dimethylbutan-2-ylidene)hydrazine hydrochloride<smiles></smiles>

Summenformel:

$\mathrm{C}_{12} \mathrm{H}_{25} \mathrm{CIN}$

Ausbeute:

$98 \%$

Molare Masse:

$232,17 \mathrm{~g} / \mathrm{mol}$

NMR:

$\delta^{1} \mathbf{H}[\mathrm{ppm}]$

$\mathrm{C}\left(\mathrm{C}_{3}\right)_{3}$

$\mathrm{CH}_{3}$

$\mathrm{N} \underline{\mathrm{H}}$

$\mathrm{CH}_{3}$

$\mathrm{C}\left(\underline{\mathrm{C}} \mathrm{H}_{3}\right)_{3}$

$\underline{\mathrm{C}}\left(\mathrm{CH}_{3}\right)_{3}$

$=\mathrm{C}_{\mathrm{t}}$

$1,19 \mathrm{~s}$.

$2,07 \mathrm{~s}$.

15,5 br.

$\delta^{13} \mathrm{C}$ [ppm]

16,2

27,3

39,8

185,9
$\mathrm{J}[\mathrm{Hz}]$ Integral

$\mathrm{J}[\mathrm{Hz}]$
18

6

1 


\section{6}

3,5-Di-tert.-butyl-2-(3,3-dimethylbut-1-en-2-yl)-1,2-diaza-3-phosphacyclopent-5-

en

oder 3,5-di-tert.-butyl-3,4-dihydro-2-(3,3-dimethylbut-1-en-2-yl)-2H-1,2,3-diazaphosphole<smiles>C=C(N1N=C(C(C)(C)C)CP1C(C)(C)C)C(C)(C)C</smiles>

Summenformel: $\quad \mathrm{C}_{16} \mathrm{H}_{31} \mathrm{~N}_{2} \mathrm{P}$

Ausbeute:

$54 \%$

Schmelzpunkt:

$108{ }^{\circ} \mathrm{C}$

Molare Masse:

$282,40 \mathrm{~g} / \mathrm{mol}$

Massenspektrum (EI): $\quad 282\left[\mathrm{C}_{16} \mathrm{H}_{31} \mathrm{~N}_{2} \mathrm{P} ; \mathrm{M}\right]^{+} 15 \%$

$267\left[\mathrm{M}-\mathrm{CH}_{3}\right]^{+} 5 \%$

$225\left[\mathrm{M}-\mathrm{C}_{4} \mathrm{H}_{9}\right]^{+} 45 \%$

$57\left[\mathrm{C}_{4} \mathrm{H}_{9}\right]^{+} 100 \%$

NMR:

$\begin{array}{lllc} & \delta^{1} \mathbf{H}[\mathbf{p p m}] & \mathbf{J}[\mathbf{H z}] & \text { Integral } \\ \mathrm{PC}\left(\underline{\mathrm{C}}_{3}\right)_{3} & 0,85 \mathrm{~d} . & { }^{3} \mathrm{~J}_{\mathrm{HP}}=11,6 & 9 \\ \mathrm{C}\left(\underline{\mathrm{CH}}_{3}\right)_{3} & 1,03 \mathrm{~s} . & & 9 \\ \mathrm{C}\left(\underline{\mathrm{CH}}_{3}\right)_{3} & 1,50 \mathrm{~s} . & & 9 \\ \mathrm{PC} \underline{\mathrm{H}}_{2}\left(\mathrm{H}_{\mathrm{a}}\right) & 2,23 \mathrm{~d} \boldsymbol{v d} . & { }^{2} \mathrm{~J}_{\mathrm{HH}}=18,7 & 1 \\ & & { }^{2} \mathrm{~J}_{\mathrm{HP}}=25,2 & \\ \mathrm{PC} \underline{\mathrm{H}}_{2}\left(\mathrm{H}_{\mathrm{b}}\right) & 2,34 \mathrm{~d} \mathbf{v d} . & { }^{2} \mathrm{~J}_{\mathrm{HH}}=18,7 & 1 \\ & & { }^{2} \mathrm{~J}_{\mathrm{HP}}=2,5 & \end{array}$




\begin{tabular}{|c|c|c|c|}
\hline & $\delta^{1} \mathrm{H}[\mathrm{ppm}]$ & $\mathbf{J}[\mathrm{Hz}]$ & Integral \\
\hline \multirow[t]{2}{*}{$=\underline{C}_{2}\left(\mathrm{H}_{\mathrm{a}}\right)$} & $4,41 \mathrm{~d} v \mathrm{~d}$ & ${ }^{2} \mathrm{~J}_{\mathrm{HH}}=0,5$ & 1 \\
\hline & & ${ }^{4} J_{H P}=1,2$ & \\
\hline \multirow[t]{3}{*}{$=\underline{C H}_{2}\left(\mathrm{H}_{\mathrm{b}}\right)$} & $5,08 \mathrm{~d} v d$ & ${ }^{2} \mathrm{~J}_{\mathrm{HH}}=0,9$ & 1 \\
\hline & & ${ }^{4} J_{H P}=5,4$ & \\
\hline & $\delta^{13} \mathrm{C}[\mathrm{ppm}]$ & $\mathbf{J}[\mathrm{Hz}]$ & \\
\hline $\mathrm{PC}\left(\underline{\mathrm{CH}}_{3}\right)_{3}$ & $25,5 \mathrm{~d}$ & ${ }^{2} \mathrm{~J}_{\mathrm{CP}}=14,7$ & \\
\hline $\mathrm{C}\left(\underline{\mathrm{CH}}_{3}\right)_{3}$ & $28,9 \mathrm{~d}$ & ${ }^{4} \mathrm{~J}_{\mathrm{CP}}=0,4$ & \\
\hline $\mathrm{P}_{\mathrm{C}} \mathrm{H}_{2}$ & $29,1 \mathrm{~d}$ & ${ }^{1} \mathrm{~J}_{\mathrm{CP}}=17,1$ & \\
\hline $\mathrm{C}\left(\underline{\mathrm{CH}}_{3}\right)_{3}$ & $30,4 \mathrm{~d}$ & ${ }^{4} \mathrm{~J}_{\mathrm{CP}}=0,5$ & \\
\hline$\underline{\mathrm{C}}\left(\mathrm{CH}_{3}\right)_{3}$ & $34,9 \mathrm{~d}$ & ${ }^{3} \mathrm{~J}_{\mathrm{CP}}=1,4$ & \\
\hline $\mathrm{P} \underline{\mathrm{C}}\left(\mathrm{CH}_{3}\right)_{3}$ & $35,0 \mathrm{~d}$ & ${ }^{1} \mathrm{~J}_{\mathrm{CP}}=27,8$ & \\
\hline$\underline{\mathrm{C}}\left(\mathrm{CH}_{3}\right)_{3}$ & 37,4 & & \\
\hline$=\underline{\mathrm{C}} \mathrm{H}_{2}$ & $88,4 \mathrm{~d}$ & ${ }^{3} \mathrm{~J}_{\mathrm{CP}}=23,2$ & \\
\hline$\underline{\mathrm{C}}_{\mathrm{t}}=\mathrm{C}$ & $155,6 \mathrm{~d}$ & ${ }^{2} \mathrm{~J}_{\mathrm{CP}}=3,7$ & \\
\hline \multirow[t]{2}{*}{$\underline{\mathrm{C}}_{\mathrm{t}}=\mathrm{N}$} & $160,5 \mathrm{~d}$ & ${ }^{2} \mathrm{~J}_{\mathrm{CP}}=11,0$ & \\
\hline & $\delta^{31} \mathrm{P}[\mathrm{ppm}]$ & $\mathrm{J}[\mathrm{Hz}]$ & \\
\hline$\underline{P}$ & $56,8 \mathrm{~s}$ & & \\
\hline
\end{tabular}




\section{7}

3,5-Di-tert-Butyl-2-(3,3-dimethylbut-1-en-2-yl)-3-oxo-1,2-Diaza-3-phosphocyclopent-5-en

oder 3,5-di-tert.-butyl-3,4-dihydro-2-(3,3-dimethylbut-1-en-2-yl)-2H-1,2,3-diazaphosphol-3-oxide<smiles>C=C(N1N=C(C(C)(C)C)CP1(=O)C(C)(C)C)C(C)(C)C</smiles>

Summenformel:

$\mathrm{C}_{16} \mathrm{H}_{31} \mathrm{~N}_{2} \mathrm{OP}$

Ausbeute:

$87 \%$

Schmelzpunkt:

$168^{\circ} \mathrm{C}$

Molare Masse:

$298,40 \mathrm{~g} / \mathrm{mol}$ 
18

(tert.-Butyl-methyl)-(tert.-butyl-((tert.-butylphosphino)methyl))ketazin<smiles>C/C(=N\N=C(/C[P+](=O)C(C)(C)C)C(C)(C)C)C(C)(C)C</smiles>

Summenformel:

$\mathrm{C}_{16} \mathrm{H}_{33} \mathrm{~N}_{2} \mathrm{OP}$

Ausbeute:

$96 \%$

Schmelzpunkt:

$121^{\circ} \mathrm{C}$

Molare Masse:

$300,42 \mathrm{~g} / \mathrm{mol}$

Massenspektrum (EI): $\quad 300\left[\mathrm{C}_{16} \mathrm{H}_{33} \mathrm{~N}_{2} \mathrm{OP} ; \mathrm{M}\right]^{+} 5 \%$

$285\left[\mathrm{M}-\mathrm{CH}_{3}\right]^{+} 5 \%$

$243\left[\mathrm{M}-\mathrm{C}_{4} \mathrm{H}_{9}\right]^{+} 100 \%$

$57\left[\mathrm{C}_{4} \mathrm{H}_{9}\right]^{+} 50 \%$

NMR:

$\delta^{1} \mathrm{H}[\mathrm{ppm}]$

$\mathbf{J}[\mathrm{Hz}]$

Integral

$\mathrm{C}\left(\mathrm{C}_{3}\right)_{3}$

$1,14 \mathrm{~s}$.

9

$\mathrm{PC}\left(\mathrm{C}_{3}\right)_{3}$

$1,17 \mathrm{~d}$.

${ }^{3} J_{H P}=16,5$

9

$\mathrm{C}\left(\mathrm{C}_{3}\right)_{3}$

$1,24 \mathrm{~s}$.

$\mathrm{CH}_{3}$

$1,90 \mathrm{~s}$.

$\mathrm{PCH}_{2}\left(\mathrm{H}_{\mathrm{a}}\right)$

$2,90 \mathrm{~d} v \mathrm{~d} v \mathrm{~d}$.

${ }^{2} \mathrm{~J}_{\mathrm{HH}}=13,1$

${ }^{2} J_{\mathrm{HP}}=17,8$

${ }^{3} \mathrm{~J}_{\mathrm{HH}}=7,8$

$\mathrm{PC}_{2}\left(\mathrm{H}_{\mathrm{b}}\right)$

3,07 dvdvd.

${ }^{2} \mathrm{~J}_{\mathrm{HH}}=13,1$

${ }^{2} \mathrm{~J}_{\mathrm{HP}}=10,7$

${ }^{3} \mathrm{~J}_{\mathrm{HH}}=0,8$ 
$\delta^{1} \mathrm{H}$ [ppm]

$\mathrm{P} \underline{\mathrm{H}}$

$6,61 \mathrm{~d} v \mathrm{~d} v \mathrm{~d}$

$\delta^{13} \mathrm{C}$ [ppm]

$13,7 \mathrm{~s}$.

23,7 d.

25, 1 d.

27,8

28,5 d.

31,9 d.

38,7

$38,8 \mathrm{~d}$.

$162,9 \mathrm{~d}$.

$171,1 \mathrm{~d}$.

$\delta^{31} \mathbf{P}$ [ppm]

$43,1 \mathrm{~s}$.

${ }^{1} \mathrm{H}$-gek. Dvdvdecvd.
J [Hz]

${ }^{1} \mathrm{~J}_{\mathrm{HP}}=463,3$

Integral

${ }^{3} \mathrm{~J}_{\mathrm{HH}}=7,8$

${ }^{3} \mathrm{~J}_{\mathrm{HH}}=0,8$

$\mathbf{J}[\mathrm{Hz}]$

${ }^{2} \mathrm{~J}_{\mathrm{CP}}=1,9$

${ }^{1} \mathrm{~J}_{\mathrm{CP}}=52,8$

${ }^{4} J_{C P}=0,9$

${ }^{1} \mathrm{~J}_{\mathrm{CP}}=66,6$

${ }^{3} \mathrm{~J}_{\mathrm{CP}}=1,3$

${ }^{2} \mathrm{~J}_{\mathrm{CP}}=10,4$

${ }^{5} J_{C P}=1,9$

J [Hz]

${ }^{1} \mathrm{~J}_{\mathrm{PH}}=463,3 \mathrm{~Hz}$

${ }^{2} \mathrm{~J}_{\mathrm{PH}}=17,8 \mathrm{~Hz}$

${ }^{3} \mathrm{~J}_{\mathrm{PH}}=16,5 \mathrm{~Hz}$

${ }^{2} \mathrm{~J}_{\mathrm{PH}}=10,7 \mathrm{~Hz}$ 


\section{9}

5-tert.-butyl-2-(3,3-dimethylbut-1-en-2-yl)-3-phenyl-1,2-diaza-3-phosphacyclopent-5-en

oder 5-di-tert.-butyl-3,4-dihydro-2-(3,3-dimethylbut-1-en-2-yl)-3-phenyl-2H-1,2,3diazaphosphole<smiles>C=C(N1N=C(C(C)(C)C)CP1c1ccccc1)C(C)(C)C</smiles>

Summenformel:

$\mathrm{C}_{18} \mathrm{H}_{27} \mathrm{~N}_{2} \mathrm{P}$

Ausbeute:

$11 \%$

Siedepunkt:

$102{ }^{\circ} \mathrm{C} / 0,005$ mbar

Molare Masse:

$302,19 \mathrm{~g} / \mathrm{mol}$

Massenspektrum (EI):

$302\left[\mathrm{C}_{18} \mathrm{H}_{27} \mathrm{~N}_{2} \mathrm{P} ; \mathrm{M}\right]^{+} 50 \%$

$287\left[\mathrm{M}-\mathrm{CH}_{3}\right]^{+} 10 \%$

$245\left[\mathrm{M}-\mathrm{C}_{4} \mathrm{H}_{9}\right]^{+} 40 \%$

$204\left[\mathrm{M}-\left(\mathrm{C}_{4} \mathrm{H}_{9}\right)\left(\mathrm{CH}_{2}\right) \mathrm{CN}\right]^{+} 15 \%$

$128\left[\left(\mathrm{C}_{4} \mathrm{H}_{9}\right)\left(\mathrm{CH}_{2}\right) \mathrm{CNP}\right]^{+} 100 \%$

$108\left[\mathrm{C}_{6} \mathrm{H}_{5} \mathrm{P}\right]^{+} 20 \%$

NMR:

$\delta^{1} \mathrm{H}$ [ppm]

$1,08 \mathrm{~s}$.

$1,30 \mathrm{~s}$.

2,59 $\underline{A B X}$
$\mathbf{J}[\mathrm{Hz}]$

$\mathrm{C}\left(\mathrm{C}_{3}\right)_{3}$

$\mathrm{C}\left(\mathrm{C}_{3}\right)_{3}$

$\mathrm{PCH}_{2}\left(\mathrm{H}_{\mathrm{a}}\right)$

\section{[}

Integral

9

9

${ }^{2} \mathrm{~J}_{\mathrm{HH}}=17,7$

1

$$
{ }^{2} \mathrm{~J}_{\mathrm{HP}}=2,4
$$




\begin{tabular}{|c|c|c|}
\hline & $\delta^{1} \mathrm{H}[\mathrm{ppm}]$ & $\mathbf{J}[\mathrm{Hz}]$ \\
\hline \multirow[t]{2}{*}{$\mathrm{PC} \underline{\mathrm{H}}_{2}\left(\mathrm{H}_{\mathrm{b}}\right)$} & $2,94 \mathrm{AB} X$ & ${ }^{2} \mathrm{~J}_{\mathrm{HH}}=17,7$ \\
\hline & & ${ }^{2} J_{H P}=26,6$ \\
\hline \multirow[t]{2}{*}{$=\underline{C}_{2}\left(\mathrm{H}_{\mathrm{a}}\right)$} & $4,14 \mathrm{~d} v \mathrm{~d}$ & ${ }^{2} \mathrm{~J}_{\mathrm{HH}}=1,1$ \\
\hline & & ${ }^{4} \mathrm{~J}_{\mathrm{HP}}=1,9$ \\
\hline \multirow[t]{2}{*}{$=\underline{C H}_{2}\left(\mathrm{H}_{\mathrm{b}}\right)$} & $4,22 \mathrm{~d} v \mathrm{~d}$ & ${ }^{2} \mathrm{~J}_{\mathrm{HH}}=1,1$ \\
\hline & & ${ }^{4} \mathrm{~J}_{\mathrm{HP}}=2,6$ \\
\hline \multirow[t]{2}{*}{$\mathrm{P}\left(\mathrm{C}_{6} \underline{\mathrm{H}}_{5}\right)$} & $7,2-7,4 \mathrm{~m}$. & \\
\hline & $\delta^{13} \mathrm{C}[\mathrm{ppm}]$ & $\mathbf{J}[\mathrm{Hz}]$ \\
\hline $\mathrm{C}\left(\underline{\mathrm{CH}}_{3}\right)_{3}$ & 28,5 & \\
\hline $\mathrm{C}\left(\underline{\mathrm{CH}}_{3}\right)_{3}$ & $29,8 \mathrm{~d}$ & ${ }^{4} \mathrm{~J}_{\mathrm{CP}}=1,8$ \\
\hline$\underline{\mathrm{C}}\left(\mathrm{CH}_{3}\right)_{3}$ & 34,8 & \\
\hline$\underline{\mathrm{C}}\left(\mathrm{CH}_{3}\right)_{3}$ & 36,7 & ${ }^{3} \mathrm{~J}_{\mathrm{CP}}=1,6$ \\
\hline $\mathrm{P}_{\mathrm{C}} \mathrm{H}_{2}$ & $37,1 \mathrm{~d}$ & ${ }^{1} \mathrm{~J}_{\mathrm{CP}}=10,3$ \\
\hline$=\underline{\mathrm{C}} \mathrm{H}_{2}$ & $88,3 \mathrm{~d}$ & ${ }^{3} J_{C P}=19,0$ \\
\hline $\mathrm{P}\left(\underline{\mathrm{C}}_{6} \mathrm{H}_{5}\right)\left(\mathrm{C}_{3}\right)$ & $128,2 \mathrm{~d}$ & ${ }^{3} \mathrm{~J}_{\mathrm{CP}}=6,2$ \\
\hline $\mathrm{P}\left(\underline{\mathrm{C}}_{6} \mathrm{H}_{5}\right)\left(\mathrm{C}_{4}\right)$ & 129,0 & \\
\hline $\mathrm{P}\left(\underline{\mathrm{C}}_{6} \mathrm{H}_{5}\right)\left(\mathrm{C}_{2}\right)$ & $129,3 \mathrm{~d}$ & ${ }^{2} J_{C P}=19,5$ \\
\hline $\mathrm{P}\left(\underline{\mathrm{C}}_{6} \mathrm{H}_{5}\right)\left(\mathrm{C}_{1}\right)$ & $139,9 \mathrm{~d}$ & ${ }^{1} \mathrm{~J}_{\mathrm{CP}}=33,3$ \\
\hline$\underline{\mathrm{C}}_{\mathrm{t}}=\mathrm{C}$ & $157,5 \mathrm{~d}$ & ${ }^{2} \mathrm{~J}_{\mathrm{CP}}=2,8$ \\
\hline \multirow[t]{2}{*}{$\underline{\mathrm{C}}_{\mathrm{t}}=\mathrm{N}$} & $159,1 \mathrm{~d}$ & ${ }^{2} \mathrm{~J}_{\mathrm{CP}}=8,5$ \\
\hline & $\delta^{31} \mathbf{P}[\mathrm{ppm}]$ & $\mathbf{J}[\mathrm{Hz}]$ \\
\hline \multirow[t]{5}{*}{$\underline{P}$} & $40,8 \mathrm{~s}$ & \\
\hline & ${ }^{1} \mathrm{H}$-gek. m., ABX & ${ }^{2} J_{P H}=26,6 \mathrm{~Hz}$ \\
\hline & & ${ }^{4} \mathrm{~J}_{\mathrm{PH}}=2,6 \mathrm{~Hz}$ \\
\hline & & ${ }^{2} \mathrm{~J}_{\mathrm{PH}}=2,4 \mathrm{~Hz}$ \\
\hline & & ${ }^{4} \mathrm{~J}_{\mathrm{PH}}=1,9 \mathrm{~Hz}$ \\
\hline
\end{tabular}




\section{1}

3-Bis-(isopropyl)amino-5-tert.-butyl-2-(3,3-dimethyl-1-(trimethylsilyl)but-1-en-2yl)-1,2-diaza-3-phosphacyclopent-5-en oder N-(5-tert.-butyl-2-(3,3-dimethyl-1-(trimethylsilyl)but-1-en-2-yl)-2H-1,2,3-diazaphosphol-3(4H)-yl)-N-isopropylpropan-2-amine<smiles>CC(C)N(C(C)C)P1CC(C(C)(C)C)=NN1/C(=C/[Si](C)(C)C)C(C)(C)C</smiles>

Summenformel:

$\mathrm{C}_{21} \mathrm{H}_{44} \mathrm{~N}_{3} \mathrm{Psi}$

Ausbeute:

$43 \%$

Schmelzpunkt:

$115^{\circ} \mathrm{C}$

Molare Masse:

$397,65 \mathrm{~g} / \mathrm{mol}$

Massenspektrum (EI): $\quad 397\left[\mathrm{C}_{21} \mathrm{H}_{44} \mathrm{~N}_{3} \mathrm{PSi} ; \mathrm{M}\right]^{+} 10 \%$

$382\left[\mathrm{M}-\mathrm{CH}_{3}\right]^{+} 10 \%$

$340\left[\mathrm{M}-\mathrm{C}_{4} \mathrm{H}_{9}\right]^{+} 100 \%$

$324\left[\mathrm{M}-\mathrm{Si}\left(\mathrm{CH}_{3}\right)_{3}\right]^{+} 1 \%$

$297\left[\mathrm{M}-\mathrm{N}\left(\mathrm{C}_{3} \mathrm{H}_{7}\right)_{2}\right]^{+} 15 \%$

$73\left[\mathrm{Si}\left(\mathrm{CH}_{3}\right)_{3}\right]^{+} 40 \%$

$57\left[\mathrm{C}_{4} \mathrm{H}_{9}\right]^{+} 20 \%$

NMR:

$\delta^{1} \mathbf{H}[\mathrm{ppm}]$

J [Hz]

Integral

$\mathrm{Si}\left(\mathrm{CH}_{3}\right)_{3}$

$0,03 d$.

${ }^{2} \mathrm{~J}_{\mathrm{Hsi}}=1,8$

9

$\mathrm{CH}\left(\mathrm{CH}_{3}\right)_{3}\left(\mathrm{CH}_{3 a}\right) \quad 1,06 \mathrm{~d}$.

${ }^{3} \mathrm{~J}_{\mathrm{HH}}=6,6$

6

$\mathrm{CH}\left(\mathrm{CH}_{3}\right)_{3}\left(\mathrm{CH}_{3 b}\right) \quad 1,10 \mathrm{~d}$.

${ }^{3} \mathrm{~J}_{\mathrm{HH}}=6,7$

6

$\mathrm{C}\left(\mathrm{C}_{3}\right)_{3}$

$1,17 \mathrm{~s}$. 
$\delta^{1} \mathrm{H}$ [ppm]

$\mathrm{C}\left(\mathrm{C}_{3}\right)_{3}$

$\mathrm{PC}_{2}\left(\mathrm{H}_{\mathrm{a}}\right)$

$\mathrm{PC}_{2}\left(\mathrm{H}_{\mathrm{b}}\right)$

$\mathrm{C} \underline{\mathrm{H}}\left(\mathrm{CH}_{3}\right)_{3}$

$=\underline{\mathrm{CH}}$

$\operatorname{SiC}\left(\mathrm{CH}_{3}\right)_{3}$

$\mathrm{CH}\left(\mathrm{CH}_{3}\right)_{2}$

$\mathrm{C}\left(\underline{\mathrm{CH}}_{3}\right)_{3}$

$\mathrm{C}\left(\underline{\mathrm{CH}}_{3}\right)_{3}$

$\mathrm{PCH}_{2}$

$\underline{\mathrm{C}}\left(\mathrm{CH}_{3}\right)_{3}$

$\mathrm{C}\left(\mathrm{CH}_{3}\right)_{3}$

$\underline{\mathrm{CH}}\left(\mathrm{CH}_{3}\right)_{2}$

$=\underline{\mathrm{CH}}$

$\underline{\mathrm{C}}_{\mathrm{t}}=\mathrm{C}$

$\underline{\mathrm{C}}_{\mathrm{t}}=\mathrm{N}$

$\underline{\text { Si }}$

$\underline{P}$
$1,23 \mathrm{~s}$.

$2,44 \underline{A B X}$

2,71 AㅁX

3,02 dvsept.

4,82 d.

$\delta^{13} \mathrm{C}$ [ppm]

$0,3 \mathrm{~d}$.

24,2

28,7

30,9

31,4 d.

34,8

38,9 d.

$47,3 \mathrm{br}$.

$107,6 \mathrm{~d}$.

$155,7 \mathrm{~d}$.

$164,7 \mathrm{~d}$.

$\delta^{29} \mathrm{Si}$ [ppm]

$-11,0 \mathrm{~d}$.

$\delta^{31} \mathrm{P}$ [ppm]

63,6 s.

${ }^{1} \mathrm{H}$-gek. ABX.
J [Hz]

Integral

9

${ }^{2} \mathrm{~J}_{\mathrm{HH}}=18,6$

1

${ }^{2} \mathrm{~J}_{\mathrm{HP}}=40,4$

${ }^{2} \mathrm{~J}_{\mathrm{HH}}=18,6$

1

${ }^{2} \mathrm{~J}_{\mathrm{HP}}=3,5$

${ }^{2} J_{H P}=9,9$

2

${ }^{3} \mathrm{~J}_{\mathrm{HH}}=6,6$

${ }^{2} J_{\mathrm{Hsi}}=3,4$

1

J [Hz]

${ }^{5} J_{C P}=9,6$

${ }^{1} \mathrm{~J}_{\mathrm{CP}}=9,0$

${ }^{3} J_{C P}=0,6$

${ }^{3} J_{C P}=9,4$

${ }^{2} \mathrm{~J}_{\mathrm{CP}}=2,5$

${ }^{2} J_{C P}=8,3$

J [Hz]

${ }^{4} J_{\text {SiP }}=1,8$

$\mathbf{J}[\mathrm{Hz}]$

${ }^{2} \mathrm{~J}_{\mathrm{PH}}=40,4$

${ }^{2} J_{\mathrm{PH}}=3,5$ 


\section{3}

5-tert-Butyl-1,2-diaza-3-phosphacyclopenta-3,5-dien oder 5-tert-butyl-2H-1,2,3-diazaphosphole<smiles>CC(C)(C)c1cp[nH]n1</smiles>

Summenformel: $\quad \mathrm{C}_{6} \mathrm{H}_{11} \mathrm{~N}_{2} \mathrm{P}$

Ausbeute: $\quad 57 \%$

Sublimationspunkt: $\quad 42{ }^{\circ} \mathrm{C} / 0,05 \mathrm{mbar}$

Molare Masse: $\quad 142,14 \mathrm{~g} / \mathrm{mol}$

Massenspektrum (EI): $\quad 142\left[\mathrm{C}_{6} \mathrm{H}_{11} \mathrm{~N}_{2} \mathrm{P} ; \mathrm{M}\right]^{+} 25 \%$

$141[\mathrm{M}-\mathrm{H}]^{+} 5 \%$

$127\left[\mathrm{M}-\mathrm{CH}_{3}\right]^{+} 100 \%$

NMR:

$\delta^{1} \mathbf{H}[\mathrm{ppm}]$

$\mathrm{C}\left(\mathrm{C}_{3}\right)_{3}$

$\mathrm{N} \underline{\mathrm{H}}$

$\mathrm{PCH}$

$\mathrm{C}\left(\underline{\mathrm{C}} \mathrm{H}_{3}\right)_{3}$

$\underline{\mathrm{C}}\left(\mathrm{CH}_{3}\right)_{3}$

$\mathrm{P} \underline{\mathrm{CH}}$

$=\mathrm{C}_{\mathrm{t}}$

$\underline{P}$

$1,36 \mathrm{~s}$.

10,6 sbr.

$7,69 \mathrm{~d}$.

$\delta^{13} \mathrm{C}$ [ppm]

$30,8 \mathrm{~d}$.

$33,7 \mathrm{~d}$.

$131,8 \mathrm{~d}$.

$169,4 \mathrm{~d}$.

$\delta^{31} \mathrm{P}$ [ppm]

222,8 s.
${ }^{1} \mathrm{H}$-gek. d.
$\mathrm{J}[\mathrm{Hz}]$

Integral

9

1

${ }^{2} \mathrm{~J}_{\mathrm{HP}}=45,6$

1

J [Hz]

${ }^{4} J_{C P}=0,8$

${ }^{3} \mathrm{~J}_{\mathrm{CP}}=1,7$

${ }^{1} J_{C P}=34,5$

${ }^{2} J_{C P}=9,7$

$\mathrm{J}[\mathrm{Hz}]$

${ }^{2} J_{P H}=45,6$ 


\section{5}

Hexakis-(3,3-dimethyl-2-(5-tert.-Butyl-1,2-diaza-3-phosphacyclopenta-3,5-dien2-yl)-but-1-en-1-yl)-cyclohexaphosphan

oder 2-(1-(2,3,4,5,6-pentakis(-2-(5-tert.-butyl-2H-1,2,3-diazyposphol-2-yl)-3,3-dimethylbut-1-enyl)-hexaphosphinan-1-yl)-3,3-dimethylbut-1-en-2-yl)-5-tert.-butyl2H-1,2,3-diazaphosphole<smiles>[R][R]1P([R])P([R])P([R])P([R])P1[R]</smiles><smiles></smiles>

Summenformel:

$\mathrm{C}_{72} \mathrm{H}_{120} \mathrm{~N}_{12} \mathrm{P}_{12}$

Ausbeute:

$5 \%$

Molare Masse:

$1525,49 \mathrm{~g} / \mathrm{mol}$

Massenspektrum (El): $\quad 762\left[\mathrm{C}_{36} \mathrm{H}_{60} \mathrm{~N}_{6} \mathrm{P}_{6} ; 3 / 6 \mathrm{M}\right]^{+} 1 \%$

$508\left[\mathrm{C}_{24} \mathrm{H}_{40} \mathrm{~N}_{4} \mathrm{P}_{4} ; 2 / 6 \mathrm{M}\right]^{+} 5 \%$

$254\left[\mathrm{C}_{12} \mathrm{H}_{20} \mathrm{~N}_{2} \mathrm{P}_{2} ; 1 / 6 \mathrm{M}\right]^{+} 100 \%$

$57\left[\mathrm{C}_{4} \mathrm{H}_{9}\right]^{+} 5 \%$

NMR:

[keine Daten, da nicht löslich in $\mathrm{C}_{6} \mathrm{D}_{6}, \mathrm{THF}-\mathrm{D}_{4}, \mathrm{CDCl}_{3}, \mathrm{D}_{2} \mathrm{O}, \mathrm{CS}_{2}$ ] 


\subsection{Röntgenstrukturdaten}

Im Nachfolgenden sind die gesammelten röntgenspektroskopischen Daten der Verbindungen IX, X, XV, $\underline{\mathbf{5}}, \underline{\mathbf{7}}, \underline{\mathbf{1 4}}, \underline{\mathbf{1 5}}, \underline{\mathbf{1 7}}, \underline{\mathbf{2 1}}$ und $\underline{\mathbf{2 5}}$ aufgeführt. In den Tabellen finden sich charakteristische Angaben zu den Kristallen und Messungen. Daran schlieBen sich für jede Verbindung ein Ortep-Plot der anisotropen Auslenkungsparameter mit 50 \%iger Wahrscheinlichkeit und je eine Tabelle für Bindungslängen und -winkel an. 


\begin{tabular}{|c|c|c|c|}
\hline Verbindung & IX & $\mathrm{X}$ & $X V$ \\
\hline Temperatur [K] & $200(2)$ & $203(2)$ & $293(2)$ \\
\hline Wellenlänge [pm] & 71,073 & 71,073 & 71,073 \\
\hline Kristallsystem & Monoklin & Monoklin & Triklin \\
\hline Raumgruppe & $\mathrm{P} 2(1) / \mathrm{C}$ & $\mathrm{P} 2(1) / \mathrm{c}$ & $P-1$ \\
\hline Zelldimensionen (a) & $2025,6(4) \mathrm{pm}$ & $1160,8(4) \mathrm{pm}$ & $1028,0(5) \mathrm{pm}$ \\
\hline (b) & $627,7(1) \mathrm{pm}$ & $614,7(1) \mathrm{pm}$ & $1216,3(5) \mathrm{pm}$ \\
\hline (c) & $1927,7(4) \mathrm{pm}$ & $2222,0(8) \mathrm{pm}$ & 2631(4) pm \\
\hline$(\alpha)$ & $90^{\circ}$ & $90^{\circ}$ & $79,50(7)^{\circ}$ \\
\hline$(\beta)$ & $112,10(3)^{\circ}$ & $100,76(3)^{\circ}$ & $89,70(4)^{\circ}$ \\
\hline$(\mathrm{y})$ & $90^{\circ}$ & $90^{\circ}$ & $77,15(3)^{\circ}$ \\
\hline Zellvolumen $\left[\mathrm{nm}^{3}\right]$ & $2,2708(8)$ & $1,5577(8)$ & $3,15(1)$ \\
\hline Z & 4 & 4 & 6 \\
\hline Dichte (ber.) $\left[\mathrm{Mg} / \mathrm{m}^{3}\right]$ & 0,997 & 1,181 & 1,050 \\
\hline Absorptionskoeffizient & $0,157 \mathrm{~mm}^{-1}$ & $0,399 \mathrm{~mm}^{-1}$ & $0,066 \mathrm{~mm}^{-1}$ \\
\hline$F(000)$ & 760 & 592 & 1092 \\
\hline Kristallgröße $\left[\mathrm{mm}^{3}\right]$ & $1,00 \times 1,00 \times 0,40$ & $0,80 \times 0,40 \times 0,40$ & $1,00 \times 0,70 \times 0,60$ \\
\hline Gem. $\theta$-Bereich & $3,69^{\circ}-25,01^{\circ}$ & $3,57^{\circ}-25,06^{\circ}$ & $3,52^{\circ}-25,02^{\circ}$ \\
\hline Indexgrenzen (h) & $-23 \leq h \leq 24$ & $-13 \leq h \leq 13$ & $-12 \leq h \leq 12$ \\
\hline$(k)$ & $-7 \leq k \leq 5$ & $-6 \leq k \leq 7$ & $-14 \leq k \leq 14$ \\
\hline (I) & $-22 \leq \mathrm{I} \leq 22$ & $-24 \leq \mathrm{I} \leq 26$ & $-31 \leq \mathrm{I} \leq 31$ \\
\hline Anzahl Reflexe & 4585 & 3339 & 17691 \\
\hline Unabhängige Reflexe & $\begin{array}{c}3997 \\
{[R \text { (int) }=0,0995]}\end{array}$ & $\begin{array}{c}2750 \\
{[R \text { (int) }=0,0539]}\end{array}$ & $\begin{array}{c}10955 \\
{[R(\text { int })=0,0551]}\end{array}$ \\
\hline Vollst. Zu $\theta \approx 25^{\circ}$ & $99,5 \%$ & $99,2 \%$ & $98.3 \%$ \\
\hline Max. \& min. Transm. & $0,9399-0,8588$ & $0,8567-0,7407$ & $0,9616-0,9371$ \\
\hline $\begin{array}{l}\text { Daten / Restraints / } \\
\text { Parameter }\end{array}$ & 3997 / 0 / 211 & $2750 / 0 / 161$ & 10955 / 0 / 680 \\
\hline Goodness-of-fit an $\mathrm{F}^{2}$ & 1,072 & 0,666 & 1,020 \\
\hline $\begin{array}{l}\text { Endgültige R-Werte } \\
\qquad[1>2 \sigma(I)]\end{array}$ & $\begin{array}{c}\mathrm{R} 1=0,0572 \\
\mathrm{wR} 2=0,1613\end{array}$ & $\begin{array}{c}\mathrm{R} 1=0,0485 \\
\mathrm{wR} 2=0,1397\end{array}$ & $\begin{aligned} R 1 & =0,0770 \\
w R 2 & =0,02391\end{aligned}$ \\
\hline $\begin{array}{l}\text { R-Werte (sämtlicher } \\
\text { Daten) }\end{array}$ & $\begin{array}{c}\mathrm{R} 1=0,0786 \\
\mathrm{wR} 2=0,1613\end{array}$ & $\begin{array}{c}R 1=0,0519 \\
w R 2=0,1444\end{array}$ & $\begin{array}{c}R 1=0,1389 \\
w R 2=0,2907\end{array}$ \\
\hline $\begin{array}{l}\text { Max. Differenz }\left[e . \AA^{3}\right] \\
\quad(\max .-\min .)\end{array}$ & $0,347--0,492$ & $0,322--0,331$ & $0,283--0,326$ \\
\hline
\end{tabular}




\begin{tabular}{|c|c|c|c|}
\hline Verbindung & $\underline{5}$ & $\underline{7}$ & $\underline{14}$ \\
\hline Temperatur [K] & 133(2) & $200(2)$ & $203(2)$ \\
\hline Wellenlänge [pm] & 71,073 & 71,073 & 71,073 \\
\hline Kristallsystem & Orthorombisch & Monoklin & Monoklin \\
\hline Raumgruppe & Pbca & $\mathrm{P} 2(1) / \mathrm{c}$ & $\mathrm{P} 2(1) / \mathrm{n}$ \\
\hline Zelldimensionen (a) & $1182,7(2) \mathrm{pm}$ & $1602,6(2) \mathrm{pm}$ & $1147,7(2) \mathrm{pm}$ \\
\hline (b) & $1182,7(2) \mathrm{pm}$ & $1231,0(2) \mathrm{pm}$ & $1127,7(2) \mathrm{pm}$ \\
\hline (c) & $2716,9(5) \mathrm{pm}$ & $1177,7(2) \mathrm{pm}$ & $1289,3(3) \mathrm{pm}$ \\
\hline$(\alpha)$ & $90^{\circ}$ & $90^{\circ}$ & $90^{\circ}$ \\
\hline$(\beta)$ & $90^{\circ}$ & $107,751(13)^{\circ}$ & $101,26(2)^{\circ}$ \\
\hline$(\mathrm{Y})$ & $90^{\circ}$ & $90^{\circ}$ & $90^{\circ}$ \\
\hline Zellvolumen $\left[\mathrm{nm}^{3}\right]$ & $3,8000(10)$ & $2,2127(5)$ & $1,6265(5)$ \\
\hline Z & 8 & 4 & 4 \\
\hline Dichte (ber.) $\left[\mathrm{Mg} / \mathrm{m}^{3}\right]$ & 1,001 & 1,022 & 1,328 \\
\hline Absorptionskoeffizient & $0,057 \mathrm{~mm}^{-1}$ & $0,058 \mathrm{~mm}^{-1}$ & $0,579 \mathrm{~mm}^{-1}$ \\
\hline$F(000)$ & 880 & 752 & 688 \\
\hline Kristallgröße $\left[\mathrm{mm}^{3}\right]$ & $0,30 \times 0,30 \times 0,10$ & $1,00 \times 0,40 \times 0,30$ & $1,00 \times 0,80 \times 0,80$ \\
\hline Gem. $\theta$-Bereich & $2,55^{\circ}-24,70^{\circ}$ & $3,57^{\circ}-22,48^{\circ}$ & $3,61^{\circ}-25,11^{\circ}$ \\
\hline Indexgrenzen (h) & $-13 \leq h \leq 6$ & $-17 \leq h \leq 17$ & $-13 \leq h \leq 13$ \\
\hline$(\mathrm{k})$ & $-13 \leq k \leq 13$ & $-13 \leq k \leq 11$ & $-10 \leq k \leq 13$ \\
\hline$(\mathrm{l})$ & $-31 \leq 1 \leq 30$ & $-12 \leq \mathrm{I} \leq 12$ & $-14 \leq \mathrm{I} \leq 15$ \\
\hline Anzahl Reflexe & 9468 & 3762 & 3381 \\
\hline Unabhängige Reflexe & $\begin{array}{c}2981 \\
{[R \text { (int) }=0,1738]}\end{array}$ & $\begin{array}{c}2875 \\
{[R \text { (int) }=0,0578]}\end{array}$ & $\begin{array}{c}2905 \\
{[R(\text { int })=0,0312]}\end{array}$ \\
\hline Vollst. Zu $\theta \approx 25^{\circ}$ & $92,1 \%$ & $99,3 \%$ & $99,4 \%$ \\
\hline Max. \& min. Transm. & $0,9943-0,9830$ & $0,9828-0,9441$ & $0,6547-0,5954$ \\
\hline $\begin{array}{l}\text { Daten / Restraints / } \\
\text { Parameter }\end{array}$ & $2981 / 0$ / 199 & $2875 / 0 / 236$ & $2905 / 0 / 169$ \\
\hline Goodness-of-fit an $\mathrm{F}^{2}$ & 1,222 & 1,026 & 1,065 \\
\hline $\begin{array}{l}\text { Endgültige R-Werte } \\
\qquad[1>2 \sigma(I)]\end{array}$ & $\begin{array}{c}R 1=0,1491 \\
w R 2=0,3682\end{array}$ & $\begin{array}{c}R 1=0,0442 \\
w R 2=0,1103\end{array}$ & $\begin{array}{c}R 1=0,0470 \\
w R 2=0,1349\end{array}$ \\
\hline $\begin{array}{l}\text { R-Werte (sämtlicher } \\
\text { Daten) }\end{array}$ & $\begin{array}{c}R 1=0,2390 \\
w R 2=0,4143\end{array}$ & $\begin{array}{c}R 1=0,0546 \\
w R 2=0,1196\end{array}$ & $\begin{array}{c}R 1=0,0485 \\
w R 2=0,1367\end{array}$ \\
\hline $\begin{array}{l}\text { Max. Differenz }\left[e . \AA^{3}\right] \\
\quad \text { (max. - min.) }\end{array}$ & $0,844--0,306$ & $0,188--0,168$ & $1,639--0,410$ \\
\hline
\end{tabular}




\begin{tabular}{|c|c|c|c|}
\hline Verbindung & $\underline{15}$ & $\underline{17}$ & $\underline{21}$ \\
\hline Temperatur [K] & $200(2)$ & $293(2)$ & 133(2) \\
\hline Wellenlänge [pm] & 71,073 & 71,073 & 71,073 \\
\hline Kristallsystem & Orthorombisch & Monoklin & Monoklin \\
\hline Raumgruppe & Pna2(1)/c & $\mathrm{Cc}$ & $\mathrm{P} 2(1) / \mathrm{c}$ \\
\hline Zelldimensionen (a) & $2155,0(8) \mathrm{pm}$ & $1018,28(18) \mathrm{pm}$ & $1072,0(1) \mathrm{pm}$ \\
\hline (b) & $639,7(2) \mathrm{pm}$ & $1724,4(3) \mathrm{pm}$ & $1342,4(1) \mathrm{pm}$ \\
\hline (c) & $1064,9(3) \mathrm{pm}$ & $1116,9(2) \mathrm{pm}$ & $1822,2(1) \mathrm{pm}$ \\
\hline$(\alpha)$ & $90^{\circ}$ & $90^{\circ}$ & $90^{\circ}$ \\
\hline$(\beta)$ & $90^{\circ}$ & $113,771(6)^{\circ}$ & $101,555(5)^{\circ}$ \\
\hline$(\mathrm{Y})$ & $90^{\circ}$ & $90^{\circ}$ & $90^{\circ}$ \\
\hline Zellvolumen $\left[\mathrm{nm}^{3}\right]$ & $1,4682(8)$ & $1,7948(6)$ & $2,5691(3)$ \\
\hline Z & 4 & 4 & 4 \\
\hline Dichte (ber.) $\left[\mathrm{Mg} / \mathrm{m}^{3}\right]$ & 1,053 & 1,156 & 1,028 \\
\hline Absorptionskoeffizient & $0,237 \mathrm{~mm}^{-1}$ & $0,157 \mathrm{~mm}^{-1}$ & $0,163 \mathrm{~mm}^{-1}$ \\
\hline$F(000)$ & 512 & 684 & 880 \\
\hline Kristallgröße $\left[\mathrm{mm}^{3}\right]$ & $1,00 \times 1,00 \times 0,80$ & $0,30 \times 0,30 \times 0,20$ & $0,50 \times 0,30 \times 0,20$ \\
\hline Gem. $\theta$-Bereich & $3,70^{\circ}-25,04^{\circ}$ & $2,36^{\circ}-27,70^{\circ}$ & $1,90^{\circ}-24,78^{\circ}$ \\
\hline Indexgrenzen (h) & $-25 \leq \mathrm{h} \leq 25$ & $-13 \leq h \leq 13$ & $-12 \leq \mathrm{h} \leq 12$ \\
\hline$(\mathrm{k})$ & $-7 \leq k \leq 7$ & $-22 \leq k \leq 22$ & $-15 \leq k \leq 14$ \\
\hline (I) & $-12 \leq \mathrm{I} \leq 12$ & $-11 \leq \mathrm{I} \leq 14$ & $-20 \leq 1 \leq 21$ \\
\hline Anzahl Reflexe & 2754 & 10797 & 9630 \\
\hline Unabhängige Reflexe & $\begin{array}{c}2604 \\
{[R \text { (int) }=0,0567]}\end{array}$ & $\begin{array}{c}3387 \\
{[R(\text { int })=0,1008]}\end{array}$ & $\begin{array}{c}4143 \\
{[R \text { (int) }=0,0358]}\end{array}$ \\
\hline Vollst. Zu $\theta \approx 25^{\circ}$ & $99,8 \%$ & $97,6 \%$ & $93,9 \%$ \\
\hline Max. \& min. Transm. & $0,8329-0,7973$ & $0,9681-0,9229$ & $0,9681-0,9229$ \\
\hline $\begin{array}{l}\text { Daten / Restraints / } \\
\text { Parameter }\end{array}$ & $2604 / 1 / 148$ & 3387 / 2 / 190 & $4143 / 0 / 248$ \\
\hline Goodness-of-fit an $\mathrm{F}^{2}$ & 0,666 & 1,375 & 1,046 \\
\hline $\begin{array}{l}\text { Endgültige R-Werte } \\
\qquad[I>2 \sigma(I)]\end{array}$ & $\begin{array}{c}\mathrm{R} 1=0,0533 \\
\mathrm{wR} 2=0,1317\end{array}$ & $\begin{array}{c}\mathrm{R} 1=0,1440 \\
\mathrm{wR} 2=0,2868\end{array}$ & $\begin{array}{c}R 1=0,0361 \\
w R 2=0,0927\end{array}$ \\
\hline $\begin{array}{l}\text { R-Werte (sämtlicher } \\
\text { Daten) }\end{array}$ & $\begin{array}{c}R 1=0,0541 \\
w R 2=0,1330\end{array}$ & $\begin{array}{c}R 1=0,1703 \\
w R 2=0,2956\end{array}$ & $\begin{array}{c}R 1=0,0460 \\
w R 2=0,0973\end{array}$ \\
\hline $\begin{array}{l}\text { Max. Differenz }\left[e . \AA^{3}\right] \\
\quad \text { (max. - min.) }\end{array}$ & $0,254--0,384$ & $0,566--0,550$ & $0,310--0,217$ \\
\hline
\end{tabular}




\begin{tabular}{|c|c|}
\hline Verbindung & $\underline{25}$ \\
\hline Temperatur [K] & $140(2)$ \\
\hline Wellenlänge [pm] & 71,073 \\
\hline Kristallsystem & Monoklin \\
\hline Raumgruppe & $\mathrm{C} 2 / \mathrm{c}$ \\
\hline Zelldimensionen (a) & $1978,4(4) \mathrm{pm}$ \\
\hline (b) & $2408,8(5) \mathrm{pm}$ \\
\hline (c) & $2121,6(4) \mathrm{pm}$ \\
\hline$(\alpha)$ & $90^{\circ}$ \\
\hline$(\beta)$ & $117,79(3)^{\circ}$ \\
\hline$(\gamma)$ & $90^{\circ}$ \\
\hline Zellvolumen $\left[\mathrm{nm}^{3}\right]$ & $8,95(1)$ \\
\hline Z & 4 \\
\hline Dichte (ber.) $\left[\mathrm{Mg} / \mathrm{m}^{3}\right]$ & 1,133 \\
\hline Absorptionskoeffizient & $0,271 \mathrm{~mm}^{-1}$ \\
\hline$F(000)$ & 3264 \\
\hline Kristallgröße $\left[\mathrm{mm}^{3}\right]$ & $0,40 \times 0,20 \times 0,20$ \\
\hline Gem. $\theta$-Bereich & $1,69^{\circ}-24,75^{\circ}$ \\
\hline Indexgrenzen (h) & $-23 \leq h \leq 23$ \\
\hline (k) & $-28 \leq k \leq 28$ \\
\hline (l) & $-24 \leq \mathrm{I} \leq 24$ \\
\hline Anzahl Reflexe & 62590 \\
\hline Unabhängige Reflexe & $\begin{array}{c}7575 \\
{[R \text { (int) }=0,0700]}\end{array}$ \\
\hline Vollst. Zu $\theta \approx 25^{\circ}$ & $98,8 \%$ \\
\hline Max. \& min. Transm. & $0,9478-0,8994$ \\
\hline $\begin{array}{l}\text { Daten / Restraints / } \\
\text { Parameter }\end{array}$ & 7575 / 96 / 483 \\
\hline Goodness-of-fit an $\mathrm{F}^{2}$ & 1,036 \\
\hline $\begin{array}{l}\text { Endgültige R-Werte } \\
\qquad[\mid>2 \sigma(I)]\end{array}$ & $\begin{array}{c}R 1=0,0377 \\
w R 2=0,0884\end{array}$ \\
\hline $\begin{array}{l}\text { R-Werte (sämtlicher } \\
\text { Daten) }\end{array}$ & $\begin{array}{c}R 1=0,0462 \\
w R 2=0,0922\end{array}$ \\
\hline $\begin{array}{l}\text { Max. Differenz }\left[e . \AA^{3}\right] \\
\quad(\max .-\min .)\end{array}$ & $0,697--0,623$ \\
\hline
\end{tabular}




\section{Verbindung IX}

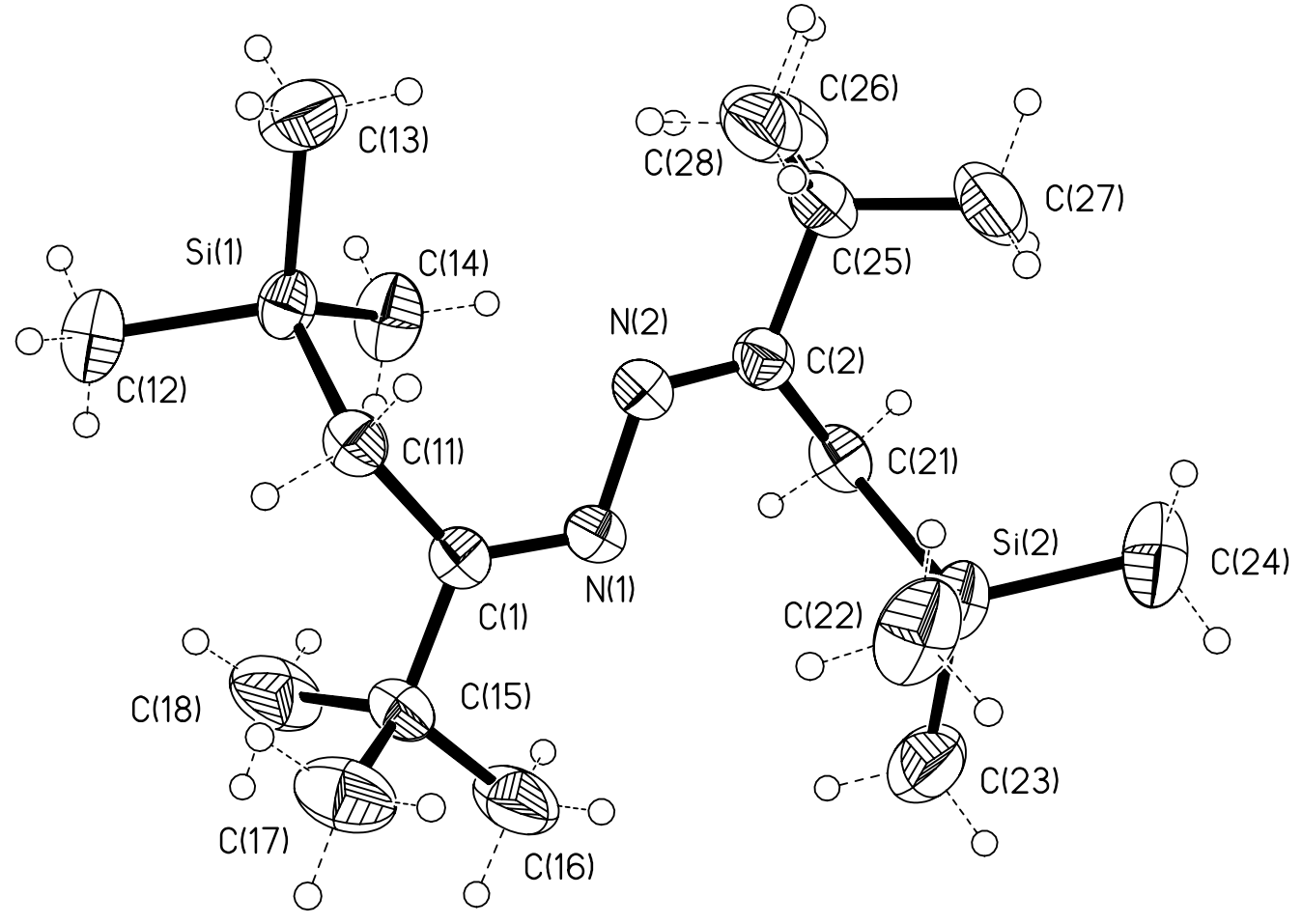

Abbildung 33: Röntgenstruktur von Verbindung IX mit anisotropen Auslenkungsparametern (50 \%)

$\begin{array}{ll}\text { Si(1)-C(13) } & 185,3(3) \\ \text { Si(1)-C(14) } & 186,4(3) \\ \text { Si(1)-C(12) } & 186,8(3) \\ \text { Si(1)-C(11) } & 189,1(2) \\ \text { Si(2)-C(23) } & 185,7(3) \\ \text { Si(2)-C(22) } & 185,9(3) \\ \text { Si(2)-C(24) } & 187,0(3) \\ \text { Si(2)-C(21) } & 189,3(2) \\ \text { N(1)-C(1) } & 128,1(3) \\ \text { N(1)-N(2) } & 142,3(3) \\ \text { N }(2)-C(2) & 128,2(3)\end{array}$

$\begin{array}{ll}\mathrm{C}(1)-\mathrm{C}(11) & 149,3(3) \\ \mathrm{C}(1)-\mathrm{C}(15) & 152,6(3) \\ \mathrm{C}(2)-\mathrm{C}(21) & 150,3(3) \\ \mathrm{C}(2)-\mathrm{C}(25) & 153,2(3) \\ \mathrm{C}(15)-\mathrm{C}(16) & 151,6(4) \\ \mathrm{C}(15)-\mathrm{C}(17) & 152,3(4) \\ \mathrm{C}(15)-\mathrm{C}(18) & 152,7(3) \\ \mathrm{C}(25)-\mathrm{C}(27) & 152,8(3) \\ \mathrm{C}(25)-\mathrm{C}(28) & 152,8(4) \\ \mathrm{C}(25)-\mathrm{C}(26) & 152,9(4)\end{array}$

Tabelle 10: Bindungslängen der Verbindung IX in pm (mit Standardabweichungen) 


\begin{tabular}{|c|c|c|c|}
\hline$C(13)-S i(1)-C(14)$ & $110,39(15)$ & $N(2)-C(2)-C(21)$ & $124,4(2)$ \\
\hline$C(13)-S i(1)-C(12)$ & $107,74(14)$ & $N(2)-C(2)-C(25)$ & $116,3(2)$ \\
\hline$C(14)-S i(1)-C(12)$ & $108,98(13)$ & $C(21)-C(2)-C(25)$ & $119,2(2)$ \\
\hline$C(13)-S i(1)-C(11)$ & $107,54(12)$ & $C(1)-C(11)-S i(1)$ & $117,52(16)$ \\
\hline$C(14)-S i(1)-C(11)$ & $111,80(12)$ & $C(16)-C(15)-C(17)$ & $108,2(2)$ \\
\hline$C(12)-S i(1)-C(11)$ & $110,31(12)$ & $C(16)-C(15)-C(1)$ & $111,8(2)$ \\
\hline$C(23)-S i(2)-C(22)$ & $110,46(17)$ & $C(17)-C(15)-C(1)$ & $109,4(2)$ \\
\hline$C(23)-S i(2)-C(24)$ & $107,56(15)$ & $C(16)-C(15)-C(18)$ & $109,6(2)$ \\
\hline$C(22)-S i(2)-C(24)$ & $108,04(14)$ & $C(17)-C(15)-C(18)$ & $108,2(2)$ \\
\hline$C(23)-S i(2)-C(21)$ & $107,93(13)$ & $C(1)-C(15)-C(18)$ & $109,52(19)$ \\
\hline$C(22)-S i(2)-C(21)$ & $110,89(12)$ & $C(2)-C(21)-S i(2)$ & $115,17(16)$ \\
\hline$C(24)-S i(2)-C(21)$ & $111,92(13)$ & $C(27)-C(25)-C(28)$ & $107,8(2)$ \\
\hline$C(1)-N(1)-N(2)$ & $113,01(19)$ & $C(27)-C(25)-C(26)$ & $110,1(3)$ \\
\hline $\mathrm{C}(2)-\mathrm{N}(2)-\mathrm{N}(1)$ & $113,11(19)$ & $C(28)-C(25)-C(26)$ & $109,4(3)$ \\
\hline$N(1)-C(1)-C(11)$ & $124,1(2)$ & $C(27)-C(25)-C(2)$ & $111,0(2)$ \\
\hline$N(1)-C(1)-C(15)$ & $116,8(2)$ & $C(28)-C(25)-C(2)$ & $111,1(2)$ \\
\hline$C(11)-C(1)-C(15)$ & $119,09(19)$ & $C(26)-C(25)-C(2)$ & $107,4(2)$ \\
\hline
\end{tabular}

Tabelle 11: Bindungswinkel der Verbindung IX in ${ }^{\circ}$ (mit Standardabweichung) 


\section{Verbindung $\mathbf{X}$}

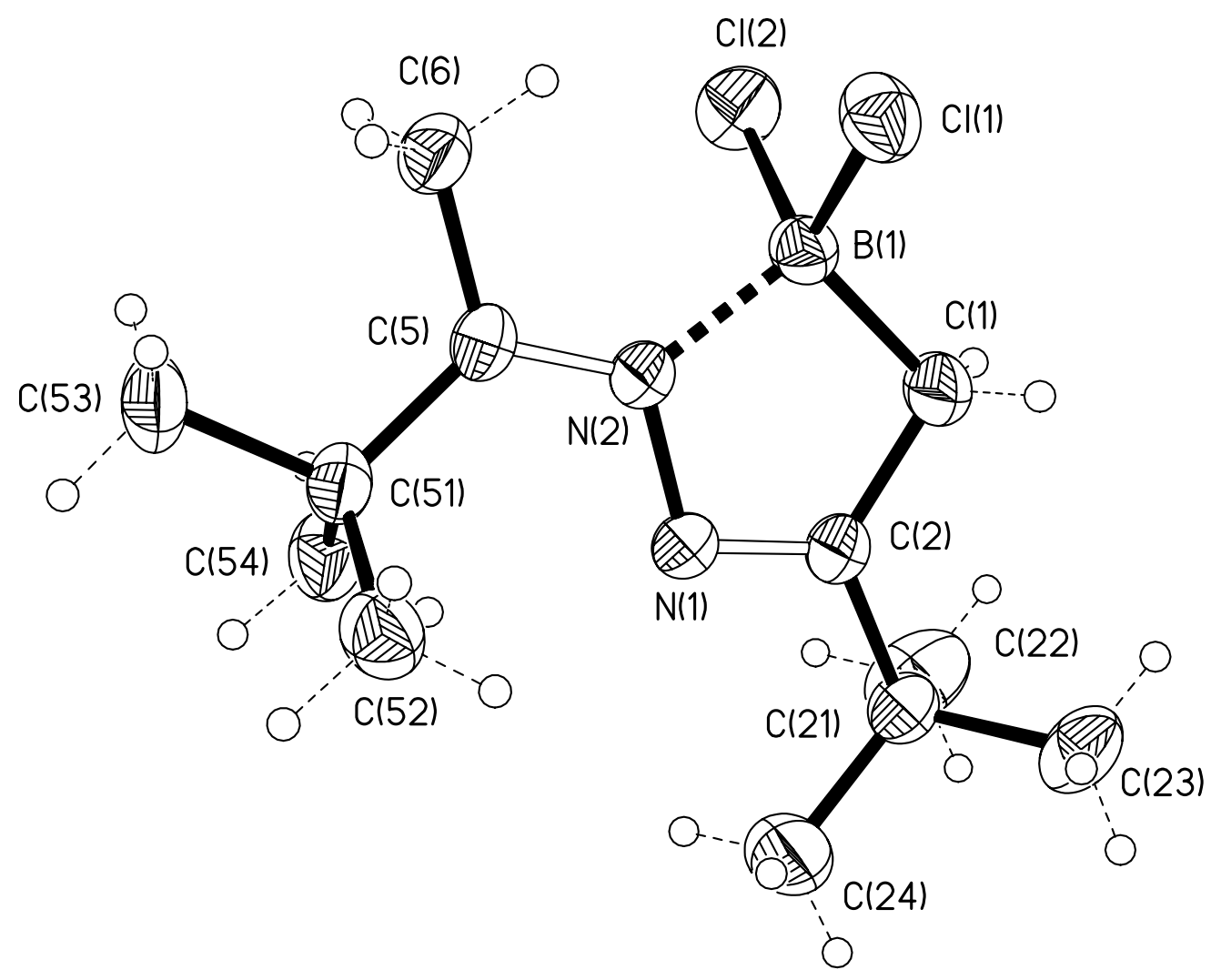

Abbildung 34: Röntgenstruktur von Verbindung X mit anisotropen Auslenkungsparametern (50 \%)

\begin{tabular}{|ll}
$\mathrm{B}(1)-\mathrm{C}(1)$ & $159,4(3)$ \\
$\mathrm{B}(1)-\mathrm{N}(2)$ & $160,9(3)$ \\
$\mathrm{B}(1)-\mathrm{Cl}(1)$ & $185,8(2)$ \\
$\mathrm{B}(1)-\mathrm{Cl}(2)$ & $185,8(3)$ \\
$\mathrm{C}(1)-\mathrm{C}(2)$ & $149,3(3)$ \\
$\mathrm{C}(2)-\mathrm{N}(1)$ & $127,9(3)$ \\
$\mathrm{C}(2)-\mathrm{C}(21)$ & $151,4(3)$ \\
$\mathrm{N}(1)-\mathrm{N}(2)$ & $142,4(2)$ \\
$\mathrm{N}(2)-\mathrm{C}(5)$ & $129,1(3)$
\end{tabular}

$\begin{array}{cc}\text { C(5)-C(6) } & 148,7(3) \\ \text { C(5)-C(51) } & 153,0(3) \\ \text { C(21)-C(24) } & 151,8(4) \\ \text { C(21)-C(22) } & 152,6(4) \\ \text { C(21)-C(23) } & 153,4(3) \\ \text { C(51)-C(54) } & 153,2(3) \\ \text { C(51)-C(52) } & 153,8(3) \\ \text { C(51)-C(53) } & 154,1(3)\end{array}$

Tabelle 12: Bindungslängen der Verbindung $\mathbf{X}$ in pm (mit Standardabweichungen) 


\begin{tabular}{|c|c|c|c|}
\hline $\mathrm{C}(1)-\mathrm{B}(1)-\mathrm{N}(2)$ & $99,67(16)$ & $N(2)-C(5)-C(51)$ & $123,35(18)$ \\
\hline $\mathrm{C}(1)-\mathrm{B}(1)-\mathrm{Cl}(1)$ & $112,92(16)$ & $C(6)-C(5)-C(51)$ & $118,85(17)$ \\
\hline $\mathrm{N}(2)-\mathrm{B}(1)-\mathrm{Cl}(1)$ & $110,97(14)$ & $C(2)-C(21)-C(24)$ & $110,92(18)$ \\
\hline $\mathrm{C}(1)-\mathrm{B}(1)-\mathrm{Cl}(2)$ & $114,13(16)$ & $C(2)-C(21)-C(22)$ & $107,7(2)$ \\
\hline $\mathrm{N}(2)-\mathrm{B}(1)-\mathrm{Cl}(2)$ & $108,15(14)$ & $C(24)-C(21)-C(22)$ & $111,5(3)$ \\
\hline $\mathrm{Cl}(1)-\mathrm{B}(1)-\mathrm{Cl}(2)$ & $110,43(12)$ & $C(2)-C(21)-C(23)$ & $109,56(18)$ \\
\hline$C(2)-C(1)-B(1)$ & $103,04(17)$ & $C(24)-C(21)-C(23)$ & $108,9(2)$ \\
\hline$N(1)-C(2)-C(1)$ & $117,67(18)$ & $C(22)-C(21)-C(23)$ & $108,2(2)$ \\
\hline$N(1)-C(2)-C(21)$ & $118,89(18)$ & $C(5)-C(51)-C(54)$ & $110,09(18)$ \\
\hline$C(1)-C(2)-C(21)$ & $123,42(18)$ & $C(5)-C(51)-C(52)$ & $109,73(17)$ \\
\hline $\mathrm{C}(2)-\mathrm{N}(1)-\mathrm{N}(2)$ & $109,49(16)$ & $C(54)-C(51)-C(52)$ & $111,31(19)$ \\
\hline $\mathrm{C}(5)-\mathrm{N}(2)-\mathrm{N}(1)$ & $118,34(16)$ & $C(5)-C(51)-C(53)$ & $110,78(18)$ \\
\hline $\mathrm{C}(5)-\mathrm{N}(2)-\mathrm{B}(1)$ & $131,65(17)$ & $C(54)-C(51)-C(53)$ & $107,45(18)$ \\
\hline $\mathrm{N}(1)-\mathrm{N}(2)-\mathrm{B}(1)$ & $109,86(14)$ & $C(52)-C(51)-C(53)$ & $107,44(19)$ \\
\hline $\mathrm{N}(2)-\mathrm{C}(5)-\mathrm{C}(6)$ & $117,77(17)$ & & \\
\hline
\end{tabular}

Tabelle 13: Bindungswinkel der Verbindung $\mathbf{X}$ in ${ }^{\circ}$ (mit Standardabweichung) 


\section{Verbindung $\mathbf{X V}$}

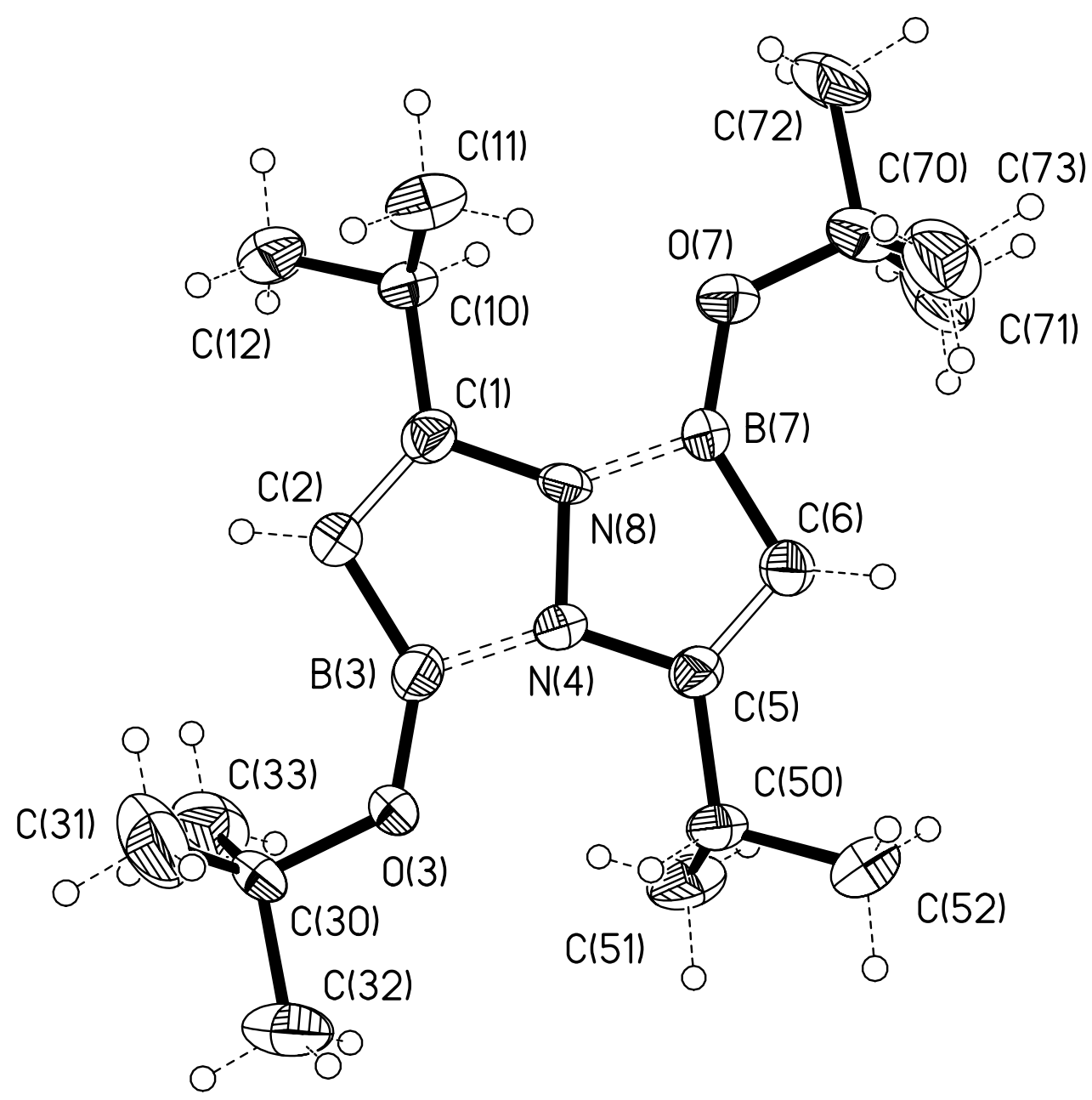

Abbildung 35: Röntgenstruktur von Verbindung XV mit anisotropen Auslenkungsparametern (50 \%)

\begin{tabular}{|cc|c|cc|}
$\mathrm{C}(1)-\mathrm{C}(2)$ & $135,6(6)$ & $\mathrm{B}(3)-\mathrm{N}(4)$ & $145,7(6)$ \\
$\mathrm{C}(1)-\mathrm{N}(8)$ & $140,7(5)$ & $\mathrm{N}(4)-\mathrm{C}(5)$ & $138,8(5)$ \\
$\mathrm{C}(1)-\mathrm{C}(10)$ & $150,7(6)$ & $\mathrm{N}(4)-\mathrm{N}(8)$ & $140,0(4)$ \\
$\mathrm{C}(2)-\mathrm{B}(3)$ & $153,1(6)$ & $\mathrm{C}(5)-\mathrm{C}(6)$ & $134,8(5)$ \\
$\mathrm{O}(3)-\mathrm{B}(3)$ & $134,7(6)$ & $\mathrm{C}(5)-\mathrm{C}(50)$ & $151,3(5)$ \\
$\mathrm{O}(3)-\mathrm{C}(30)$ & $146,4(5)$ & $\mathrm{C}(6)-\mathrm{B}(7)$ & $152,8(6)$
\end{tabular}

Tabelle 14: Bindungslängen der Verbindung XV in pm (mit Standardabweichungen) 


\begin{tabular}{|c|c|c|c|}
\hline $\mathrm{B}(7)-\mathrm{O}(7)$ & $135,9(5)$ & $\mathrm{C}(41)-\mathrm{N}(44)$ & $137,4(5)$ \\
\hline $\mathrm{B}(7)-\mathrm{N}(8)$ & $146,0(5)$ & $C(41)-C(410)$ & $150,6(5)$ \\
\hline $\mathrm{O}(7)-\mathrm{C}(70)$ & $144,2(5)$ & $\mathrm{C}(42)-\mathrm{B}(43) \# 2$ & $155,6(6)$ \\
\hline$C(10)-C(12)$ & $152,4(6)$ & $\mathrm{O}(43)-\mathrm{B}(43)$ & $136,4(5)$ \\
\hline$C(10)-C(11)$ & $153,3(6)$ & $\mathrm{O}(43)-\mathrm{C}(430)$ & $145,8(5)$ \\
\hline$C(21)-C(22)$ & $135,5(6)$ & $\mathrm{B}(43)-\mathrm{N}(44)$ & $143,3(6)$ \\
\hline$C(21)-N(24)$ & $138,9(5)$ & $\mathrm{B}(43)-\mathrm{C}(42) \# 2$ & $155,6(6)$ \\
\hline$C(21)-C(210)$ & $151,2(6)$ & $\mathrm{N}(44)-\mathrm{N}(44) \# 2$ & $140,3(6)$ \\
\hline$C(22)-B(27)$ & $153,1(7)$ & $C(50)-C(52)$ & $152,0(6)$ \\
\hline $\mathrm{O}(23)-\mathrm{B}(23)$ & $136,5(6)$ & $C(50)-C(51)$ & $152,7(7)$ \\
\hline $\mathrm{O}(23)-\mathrm{C}(230)$ & $145,0(5)$ & $C(70)-C(71)$ & $151,3(7)$ \\
\hline $\mathrm{B}(23)-\mathrm{N}(24)$ & $145,6(6)$ & $\mathrm{C}(70)-\mathrm{C}(73)$ & $151,7(7)$ \\
\hline$B(23)-C(26)$ & $153,3(7)$ & $C(70)-C(72)$ & $152,4(7)$ \\
\hline $\mathrm{N}(24)-\mathrm{N}(28)$ & $140,1(4)$ & $C(210)-C(211)$ & $152,6(6)$ \\
\hline$C(25)-C(26)$ & $135,3(6)$ & $C(210)-C(212)$ & $152,8(6)$ \\
\hline $\mathrm{C}(25)-\mathrm{N}(28)$ & $139,0(5)$ & $C(230)-C(233)$ & $151,5(7)$ \\
\hline$C(25)-C(250)$ & $150,1(6)$ & $C(230)-C(232)$ & $151,8(7)$ \\
\hline $\mathrm{O}(27)-\mathrm{B}(27)$ & $136,2(6)$ & $C(230)-C(231)$ & $151,9(7)$ \\
\hline $\mathrm{O}(27)-\mathrm{C}(270)$ & $145,4(5)$ & $C(250)-C(252)$ & $152,1(6)$ \\
\hline $\mathrm{B}(27)-\mathrm{N}(28)$ & $145,9(6)$ & $C(250)-C(251)$ & $152,7(6)$ \\
\hline$C(30)-C(32)$ & $150,8(6)$ & $C(270)-C(273)$ & $151,4(7)$ \\
\hline$C(30)-C(31)$ & $151,2(7)$ & $C(270)-C(271)$ & $151,6(7)$ \\
\hline$C(30)-C(33)$ & $153,1(7)$ & $\mathrm{C}(270)-\mathrm{C}(272)$ & $152,2(7)$ \\
\hline $\mathrm{O}(33)-\mathrm{B}(33)$ & $136,6(6)$ & $C(310)-C(311)$ & $152,5(6)$ \\
\hline $\mathrm{O}(33)-\mathrm{C}(330)$ & $145,7(5)$ & $C(310)-C(312)$ & $152,8(6)$ \\
\hline $\mathrm{B}(33)-\mathrm{N}(34)$ & $145,1(6)$ & $C(330)-C(333)$ & $151,1(7)$ \\
\hline$B(33)-C(34)$ & $153,0(7)$ & $C(330)-C(332)$ & $151,9(7)$ \\
\hline$C(34)-C(35) \# 1$ & $135,4(6)$ & $C(330)-C(331)$ & $152,1(7)$ \\
\hline$N(34)-C(35)$ & $139,1(5)$ & $C(410)-C(412)$ & $152,6(6)$ \\
\hline $\mathrm{N}(34)-\mathrm{N}(34) \# 1$ & $139,8(6)$ & $C(410)-C(411)$ & $152,9(7)$ \\
\hline$C(35)-C(34) \# 1$ & $135,4(6)$ & $C(430)-C(431)$ & $147,9(7)$ \\
\hline C(35)-C(310) & $151,0(6)$ & $C(430)-C(433)$ & $151,5(7)$ \\
\hline$C(41)-C(42)$ & $135,8(5)$ & $\mathrm{C}(430)-\mathrm{C}(432)$ & $152,2(7)$ \\
\hline
\end{tabular}

Fortsetzung der Bindungslängen von Verbindung XV in pm (mit Standardabweichungen) 


\begin{tabular}{|c|c|c|c|}
\hline $\mathrm{C}(2)-\mathrm{C}(1)-\mathrm{N}(8)$ & $112,3(4)$ & $\mathrm{N}(34) \# 1-\mathrm{N}(34)-\mathrm{B}(33)$ & $109,5(4)$ \\
\hline$C(2)-C(1)-C(10)$ & $130,3(4)$ & $\mathrm{C}(34) \# 1-\mathrm{C}(35)-\mathrm{N}(34)$ & $112,7(4)$ \\
\hline$N(8)-C(1)-C(10)$ & $117,4(3)$ & $\mathrm{C}(34) \# 1-C(35)-C(310)$ & $130,3(4)$ \\
\hline$C(1)-C(2)-B(3)$ & $106,7(4)$ & $N(34)-C(35)-C(310)$ & $117,0(4)$ \\
\hline $\mathrm{B}(3)-\mathrm{O}(3)-\mathrm{C}(30)$ & $125,3(3)$ & $C(42)-C(41)-N(44)$ & $111,6(3)$ \\
\hline $\mathrm{O}(3)-\mathrm{B}(3)-\mathrm{N}(4)$ & $117,6(4)$ & $C(42)-C(41)-C(410)$ & $130,9(4)$ \\
\hline $\mathrm{O}(3)-\mathrm{B}(3)-\mathrm{C}(2)$ & $138,7(4)$ & $\mathrm{N}(44)-\mathrm{C}(41)-\mathrm{C}(410)$ & $117,5(3)$ \\
\hline $\mathrm{N}(4)-\mathrm{B}(3)-\mathrm{C}(2)$ & $103,6(4)$ & $C(41)-C(42)-B(43) \# 2$ & $106,2(3)$ \\
\hline $\mathrm{C}(5)-\mathrm{N}(4)-\mathrm{N}(8)$ & $106,8(3)$ & $\mathrm{B}(43)-\mathrm{O}(43)-\mathrm{C}(430)$ & $127,6(4)$ \\
\hline $\mathrm{C}(5)-\mathrm{N}(4)-\mathrm{B}(3)$ & $143,6(4)$ & $\mathrm{O}(43)-\mathrm{B}(43)-\mathrm{N}(44)$ & $121,4(4)$ \\
\hline $\mathrm{N}(8)-\mathrm{N}(4)-\mathrm{B}(3)$ & $109,6(3)$ & $\mathrm{O}(43)-\mathrm{B}(43)-\mathrm{C}(42) \# 2$ & $135,2(4)$ \\
\hline $\mathrm{C}(6)-\mathrm{C}(5)-\mathrm{N}(4)$ & $112,9(4)$ & $\mathrm{N}(44)-\mathrm{B}(43)-\mathrm{C}(42) \# 2$ & $103,4(3)$ \\
\hline$C(6)-C(5)-C(50)$ & $131,0(4)$ & $\mathrm{C}(41)-\mathrm{N}(44)-\mathrm{N}(44) \# 2$ & $109,7(4)$ \\
\hline$N(4)-C(5)-C(50)$ & $116,1(3)$ & $\mathrm{C}(41)-\mathrm{N}(44)-\mathrm{B}(43)$ & $141,2(3)$ \\
\hline$C(5)-C(6)-B(7)$ & $107,6(4)$ & $\mathrm{N}(44) \# 2-\mathrm{N}(44)-\mathrm{B}(43)$ & $109,1(4)$ \\
\hline $\mathrm{O}(7)-\mathrm{B}(7)-\mathrm{N}(8)$ & $118,8(4)$ & $C(5)-C(50)-C(52)$ & $111,2(3)$ \\
\hline $\mathrm{O}(7)-\mathrm{B}(7)-\mathrm{C}(6)$ & $139,5(4)$ & $C(5)-C(50)-C(51)$ & $110,4(4)$ \\
\hline $\mathrm{N}(8)-\mathrm{B}(7)-\mathrm{C}(6)$ & $101,8(3)$ & $C(52)-C(50)-C(51)$ & $111,2(4)$ \\
\hline $\mathrm{B}(7)-\mathrm{O}(7)-\mathrm{C}(70)$ & $124,3(4)$ & $\mathrm{O}(7)-\mathrm{C}(70)-\mathrm{C}(71)$ & $110,0(4)$ \\
\hline $\mathrm{N}(4)-\mathrm{N}(8)-\mathrm{C}(1)$ & $107,7(3)$ & $\mathrm{O}(7)-\mathrm{C}(70)-\mathrm{C}(73)$ & $109,6(4)$ \\
\hline $\mathrm{N}(4)-\mathrm{N}(8)-\mathrm{B}(7)$ & $111,0(3)$ & $C(71)-C(70)-C(73)$ & $111,3(5)$ \\
\hline $\mathrm{C}(1)-\mathrm{N}(8)-\mathrm{B}(7)$ & $141,3(4)$ & $\mathrm{O}(7)-\mathrm{C}(70)-\mathrm{C}(72)$ & $104,3(4)$ \\
\hline$C(1)-C(10)-C(12)$ & $111,6(3)$ & $C(71)-C(70)-C(72)$ & $111,6(5)$ \\
\hline$C(1)-C(10)-C(11)$ & $110,3(4)$ & $C(73)-C(70)-C(72)$ & $109,7(5)$ \\
\hline$C(12)-C(10)-C(11)$ & $110,8(4)$ & $C(21)-C(210)-C(211)$ & $111,6(4)$ \\
\hline $\mathrm{C}(22)-\mathrm{C}(21)-\mathrm{N}(24)$ & $112,2(4)$ & $C(21)-C(210)-C(212)$ & $110,6(3)$ \\
\hline$C(22)-C(21)-C(210)$ & $130,7(4)$ & $C(211)-C(210)-C(212)$ & $111,0(4)$ \\
\hline$N(24)-C(21)-C(210)$ & $117,1(4)$ & $\mathrm{O}(23)-\mathrm{C}(230)-\mathrm{C}(233)$ & $104,1(4)$ \\
\hline $\mathrm{C}(21)-\mathrm{C}(22)-\mathrm{B}(27)$ & $106,8(4)$ & $\mathrm{O}(23)-\mathrm{C}(230)-\mathrm{C}(232)$ & $108,6(4)$ \\
\hline $\mathrm{B}(23)-\mathrm{O}(23)-\mathrm{C}(230)$ & $125,3(4)$ & $\mathrm{C}(233)-\mathrm{C}(230)-\mathrm{C}(232)$ & $111,1(4)$ \\
\hline $\mathrm{O}(23)-\mathrm{B}(23)-\mathrm{N}(24)$ & $119,3(4)$ & $\mathrm{O}(23)-\mathrm{C}(230)-\mathrm{C}(231)$ & $110,2(4)$ \\
\hline $\mathrm{O}(23)-\mathrm{B}(23)-\mathrm{C}(26)$ & $137,4(4)$ & $C(233)-C(230)-C(231)$ & $111,0(4)$ \\
\hline $\mathrm{N}(24)-\mathrm{B}(23)-\mathrm{C}(26)$ & $103,3(4)$ & $\mathrm{C}(232)-\mathrm{C}(230)-\mathrm{C}(231)$ & $111,6(5)$ \\
\hline
\end{tabular}

Tabelle 15: Bindungswinkel der Verbindung XV in ${ }^{\circ}$ (mit Standardabweichung) 


\begin{tabular}{|c|c|c|c|}
\hline $\mathrm{C}(21)-\mathrm{N}(24)-\mathrm{N}(28)$ & $108,5(3)$ & $C(25)-C(250)-C(252)$ & $111,6(4)$ \\
\hline $\mathrm{C}(21)-\mathrm{N}(24)-\mathrm{B}(23)$ & $142,2(4)$ & $C(25)-C(250)-C(251)$ & $110,4(3)$ \\
\hline$N(28)-N(24)-B(23)$ & $109,2(3)$ & $C(252)-C(250)-C(251)$ & $111,0(4)$ \\
\hline $\mathrm{C}(26)-\mathrm{C}(25)-\mathrm{N}(28)$ & $112,2(4)$ & $\mathrm{O}(27)-\mathrm{C}(270)-\mathrm{C}(273)$ & $103,9(4)$ \\
\hline$C(26)-C(25)-C(250)$ & $131,0(4)$ & $\mathrm{O}(27)-\mathrm{C}(270)-\mathrm{C}(271)$ & $109,0(4)$ \\
\hline $\mathrm{N}(28)-\mathrm{C}(25)-\mathrm{C}(250)$ & $116,8(4)$ & $\mathrm{C}(273)-\mathrm{C}(270)-\mathrm{C}(271)$ & $111,7(4)$ \\
\hline$C(25)-C(26)-B(23)$ & $106,9(4)$ & $\mathrm{O}(27)-\mathrm{C}(270)-\mathrm{C}(272)$ & $110,0(4)$ \\
\hline $\mathrm{B}(27)-\mathrm{O}(27)-\mathrm{C}(270)$ & $124,9(4)$ & $C(273)-C(270)-C(272)$ & $110,7(4)$ \\
\hline $\mathrm{O}(27)-\mathrm{B}(27)-\mathrm{N}(28)$ & $118,8(4)$ & $\mathrm{C}(271)-\mathrm{C}(270)-\mathrm{C}(272)$ & $111,3(5)$ \\
\hline $\mathrm{O}(27)-\mathrm{B}(27)-\mathrm{C}(22)$ & $137,8(4)$ & $C(35)-C(310)-C(311)$ & $110,9(3)$ \\
\hline $\mathrm{N}(28)-\mathrm{B}(27)-\mathrm{C}(22)$ & $103,4(4)$ & $C(35)-C(310)-C(312)$ & $111,7(4)$ \\
\hline $\mathrm{C}(25)-\mathrm{N}(28)-\mathrm{N}(24)$ & $108,4(3)$ & $C(311)-C(310)-C(312)$ & $110,6(4)$ \\
\hline $\mathrm{C}(25)-\mathrm{N}(28)-\mathrm{B}(27)$ & $142,5(4)$ & $\mathrm{O}(33)-\mathrm{C}(330)-\mathrm{C}(333)$ & $104,3(4)$ \\
\hline $\mathrm{N}(24)-\mathrm{N}(28)-\mathrm{B}(27)$ & $109,0(3)$ & $\mathrm{O}(33)-\mathrm{C}(330)-\mathrm{C}(332)$ & $109,2(4)$ \\
\hline $\mathrm{O}(3)-\mathrm{C}(30)-\mathrm{C}(32)$ & $105,5(4)$ & $C(333)-C(330)-C(332)$ & $111,4(4)$ \\
\hline $\mathrm{O}(3)-\mathrm{C}(30)-\mathrm{C}(31)$ & $109,9(4)$ & $\mathrm{O}(33)-\mathrm{C}(330)-\mathrm{C}(331)$ & $109,8(4)$ \\
\hline$C(32)-C(30)-C(31)$ & $112,3(5)$ & $C(333)-C(330)-C(331)$ & $110,6(4)$ \\
\hline $\mathrm{O}(3)-\mathrm{C}(30)-\mathrm{C}(33)$ & $108,7(4)$ & $C(332)-C(330)-C(331)$ & $111,3(5)$ \\
\hline$C(32)-C(30)-C(33)$ & $109,3(5)$ & $C(41)-C(410)-C(412)$ & $111,5(4)$ \\
\hline$C(31)-C(30)-C(33)$ & $110,9(5)$ & $C(41)-C(410)-C(411)$ & $110,3(4)$ \\
\hline $\mathrm{B}(33)-\mathrm{O}(33)-\mathrm{C}(330)$ & $125,0(4)$ & $C(412)-C(410)-C(411)$ & $110,8(4)$ \\
\hline $\mathrm{O}(33)-\mathrm{B}(33)-\mathrm{N}(34)$ & $118,6(4)$ & $\mathrm{O}(43)-\mathrm{C}(430)-\mathrm{C}(431)$ & $104,3(4)$ \\
\hline $\mathrm{O}(33)-\mathrm{B}(33)-\mathrm{C}(34)$ & $137,8(4)$ & $\mathrm{O}(43)-\mathrm{C}(430)-\mathrm{C}(433)$ & $108,7(4)$ \\
\hline $\mathrm{N}(34)-\mathrm{B}(33)-\mathrm{C}(34)$ & $103,6(4)$ & $C(431)-C(430)-C(433)$ & $110,9(5)$ \\
\hline $\mathrm{C}(35) \# 1-\mathrm{C}(34)-\mathrm{B}(33)$ & $106,3(4)$ & $\mathrm{O}(43)-\mathrm{C}(430)-\mathrm{C}(432)$ & $108,9(4)$ \\
\hline $\mathrm{C}(35)-\mathrm{N}(34)-\mathrm{N}(34) \# 1$ & $107,9(4)$ & $C(431)-C(430)-C(432)$ & $112,8(5)$ \\
\hline $\mathrm{C}(35)-\mathrm{N}(34)-\mathrm{B}(33)$ & $142,6(4)$ & $C(433)-C(430)-C(432)$ & $111,0(5)$ \\
\hline
\end{tabular}

Fortsetzung der Bindungswinkel von Verbindung $\mathbf{X V}$ in ${ }^{\circ}$ (mit Standardabweichung) 
Verbindung $\underline{\mathbf{5}}$

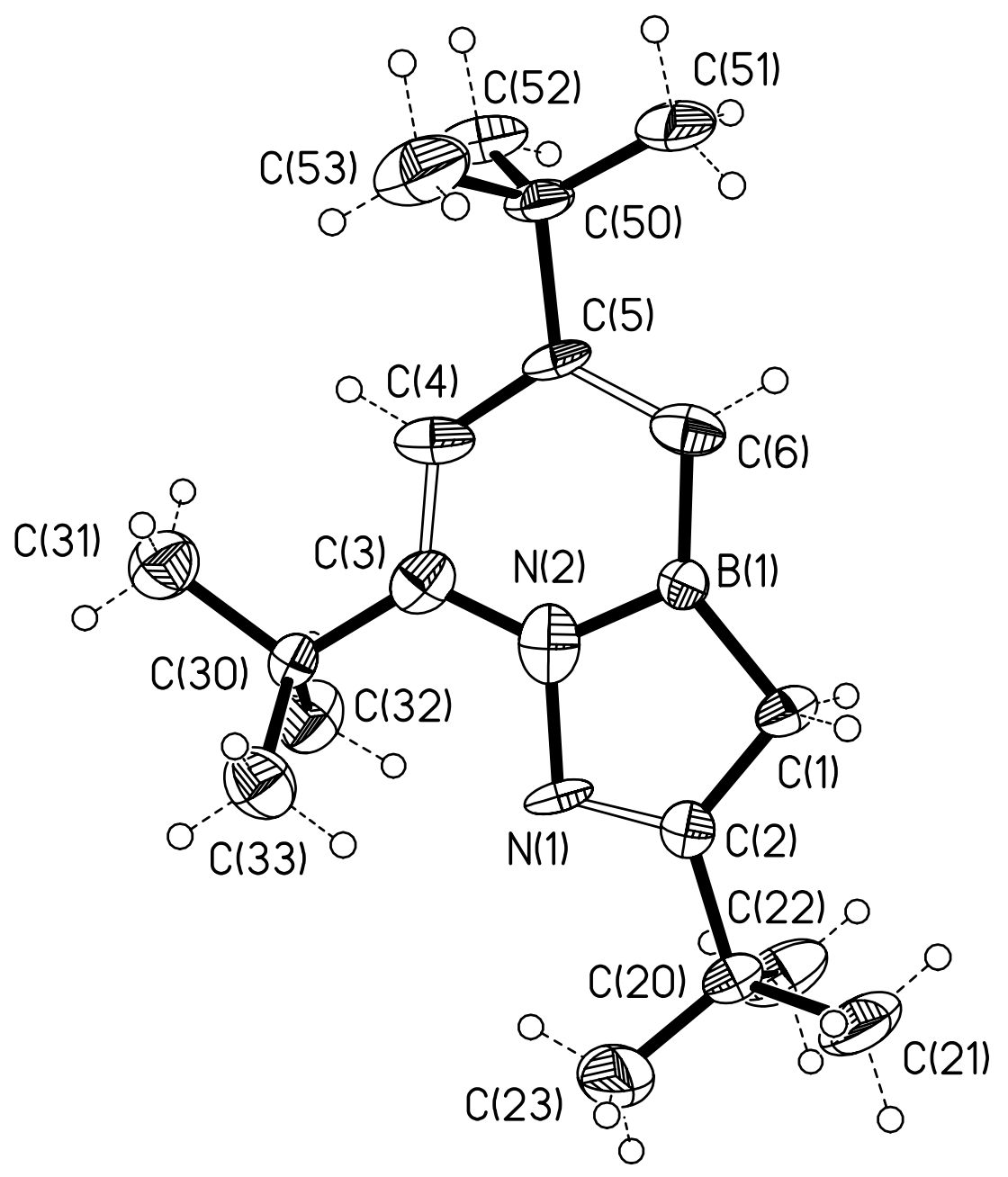

Abbildung 36: Röntgenstruktur von Verbindung $\underline{\mathbf{5}}$ mit anisotropen Auslenkungsparametern (50 \%)

\begin{tabular}{|c|c|c|c|}
\hline $\mathrm{B}(1)-\mathrm{N}(2)$ & $142,3(10)$ & $C(1)-C(2)$ & $150,7(10)$ \\
\hline$B(1)-C(6)$ & $149,1(11)$ & $C(2)-C(20)$ & $153,4(10)$ \\
\hline $\mathrm{B}(1)-\mathrm{C}(1)$ & $157,5(10)$ & $C(3)-C(4)$ & $139,1(11)$ \\
\hline$N(1)-C(2)$ & $128,5(9)$ & $C(3)-C(30)$ & $150,6(10)$ \\
\hline$N(1)-N(2)$ & $150,2(10)$ & $C(4)-C(5)$ & $141,4(10)$ \\
\hline$N(2)-C(3)$ & $135,8(10)$ & $C(5)-C(6)$ & $140,4(11)$ \\
\hline
\end{tabular}

Tabelle 16: Bindungslängen der Verbindung $\underline{\mathbf{5}}$ in pm (mit Standardabweichungen) 


$\begin{array}{|ll|}\mathrm{C}(5)-\mathrm{C}(50) & 152,0(9) \\ \mathrm{C}(20)-\mathrm{C}(23) & 147,7(12) \\ \mathrm{C}(20)-\mathrm{C}(21) & 154,5(12) \\ \mathrm{C}(20)-\mathrm{C}(22) & 156,8(11) \\ \mathrm{C}(30)-\mathrm{C}(33) & 153,9(12)\end{array} \mid \begin{array}{cc}\mathrm{C}(30)-\mathrm{C}(31) & 156,2(11) \\ \mathrm{C}(30)-\mathrm{C}(32) & 157,6(9) \\ \mathrm{C}(50)-\mathrm{C}(51) & 148,3(11) \\ \mathrm{C}(50)-\mathrm{C}(53) & 154,3(12) \\ \mathrm{C}(50)-\mathrm{C}(52) & 156,3(14)\end{array}$

Fortsetzung der Bindungslängen der Verbindung $\underline{\mathbf{5}}$ in pm (mit Standardabweichungen)

\begin{tabular}{|c|c|c|c|}
\hline$N(2)-B(1)-C(6)$ & $116,7(6)$ & $C(23)-C(20)-C(2)$ & $111,1(6)$ \\
\hline$N(2)-B(1)-C(1)$ & $104,0(6)$ & $C(23)-C(20)-C(21)$ & $112,2(9)$ \\
\hline$C(6)-B(1)-C(1)$ & $139,4(6)$ & $C(2)-C(20)-C(21)$ & $109,7(6)$ \\
\hline $\mathrm{C}(2)-\mathrm{N}(1)-\mathrm{N}(2)$ & $106,6(6)$ & $C(23)-C(20)-C(22)$ & $109,6(7)$ \\
\hline$C(3)-N(2)-B(1)$ & $129,5(8)$ & $C(2)-C(20)-C(22)$ & $106,1(7)$ \\
\hline $\mathrm{C}(3)-\mathrm{N}(2)-\mathrm{N}(1)$ & $118,7(6)$ & $C(21)-C(20)-C(22)$ & $107,8(6)$ \\
\hline $\mathrm{B}(1)-\mathrm{N}(2)-\mathrm{N}(1)$ & $111,8(6)$ & $C(3)-C(30)-C(33)$ & $112,6(6)$ \\
\hline$C(2)-C(1)-B(1)$ & $102,1(5)$ & $C(3)-C(30)-C(31)$ & $111,5(7)$ \\
\hline$N(1)-C(2)-C(1)$ & $115,5(7)$ & $C(33)-C(30)-C(31)$ & $107,5(6)$ \\
\hline$N(1)-C(2)-C(20)$ & $120,2(6)$ & $C(3)-C(30)-C(32)$ & $109,0(6)$ \\
\hline$C(1)-C(2)-C(20)$ & $124,3(6)$ & $C(33)-C(30)-C(32)$ & $109,9(7)$ \\
\hline$N(2)-C(3)-C(4)$ & $109,6(7)$ & $C(31)-C(30)-C(32)$ & $106,1(6)$ \\
\hline$N(2)-C(3)-C(30)$ & $122,5(7)$ & $C(51)-C(50)-C(5)$ & $113,0(6)$ \\
\hline$C(4)-C(3)-C(30)$ & $127,7(7)$ & $C(51)-C(50)-C(53)$ & $109,1(8)$ \\
\hline$C(3)-C(4)-C(5)$ & $129,4(7)$ & $C(5)-C(50)-C(53)$ & $108,8(7)$ \\
\hline$C(6)-C(5)-C(4)$ & $118,4(6)$ & $C(51)-C(50)-C(52)$ & $110,5(9)$ \\
\hline$C(6)-C(5)-C(50)$ & $123,6(6)$ & $C(5)-C(50)-C(52)$ & $108,7(6)$ \\
\hline$C(4)-C(5)-C(50)$ & $118,0(6)$ & $C(53)-C(50)-C(52)$ & $106,5(7)$ \\
\hline$C(5)-C(6)-B(1)$ & $116,3(6)$ & & \\
\hline
\end{tabular}

Tabelle 17: Bindungswinkel der Verbindung $\underline{\mathbf{5}}$ in ${ }^{\circ}$ (mit Standardabweichung) 


\section{Verbindung $\underline{7}$}

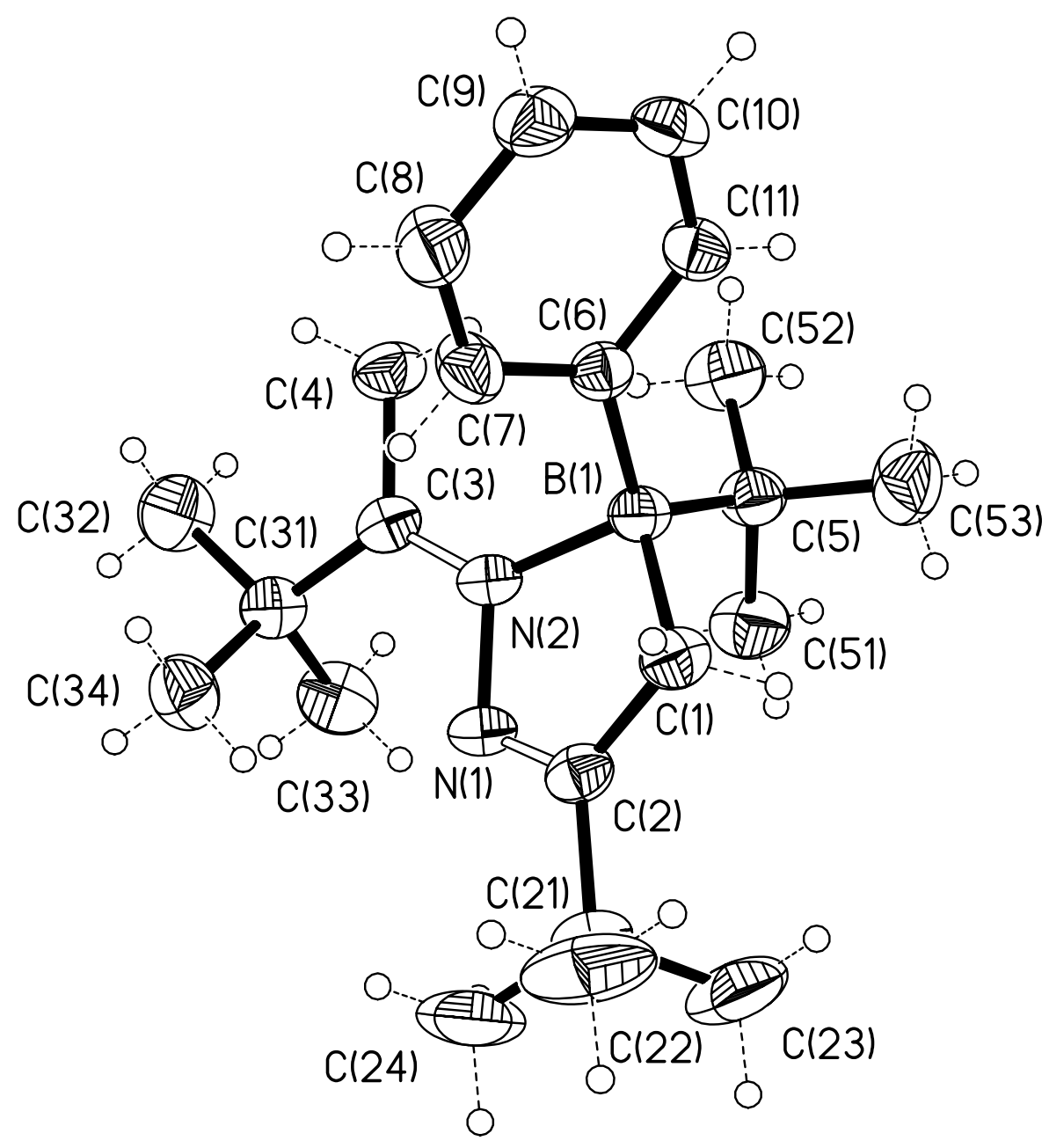

Abbildung 37: Röntgenstruktur von Verbindung $\underline{7}$ mit anisotropen Auslenkungsparametern (50 \%)

$\begin{array}{|ll|}\mathrm{B}(1)-\mathrm{C}(6) & 162,8(3) \\ \mathrm{B}(1)-\mathrm{C}(1) & 163,7(3) \\ \mathrm{B}(1)-\mathrm{C}(5) & 165,8(3) \\ \mathrm{B}(1)-\mathrm{N}(2) & 167,3(3) \\ \mathrm{C}(1)-\mathrm{C}(2) & 148,2(3) \\ \mathrm{N}(1)-\mathrm{C}(2) & 128,2(2)\end{array} \mid \begin{array}{cc}\mathrm{N}(1)-\mathrm{N}(2) & 143,1(2) \\ \mathrm{C}(2)-\mathrm{C}(21) & 152,2(3) \\ \mathrm{N}(2)-\mathrm{C}(3) & 129,5(2) \\ \mathrm{C}(3)-\mathrm{C}(4) & 149,3(2) \\ \mathrm{C}(3)-\mathrm{C}(31) & 153,5(3) \\ \mathrm{C}(5)-\mathrm{C}(51) & 154,1(3)\end{array}$

Tabelle 18: Bindungslängen der Verbindung $\underline{7}$ in pm (mit Standardabweichungen) 


\begin{tabular}{|ll}
$\mathrm{C}(5)-\mathrm{C}(52)$ & $153,2(3)$ \\
$\mathrm{C}(5)-\mathrm{C}(53)$ & $154,5(3)$ \\
$\mathrm{C}(6)-\mathrm{C}(11)$ & $139,4(3)$ \\
$\mathrm{C}(6)-\mathrm{C}(7)$ & $139,9(3)$ \\
$\mathrm{C}(7)-\mathrm{C}(8)$ & $138,8(3)$ \\
$\mathrm{C}(8)-\mathrm{C}(9)$ & $137,7(3)$ \\
$\mathrm{C}(9)-\mathrm{C}(10)$ & $137,0(3)$
\end{tabular} \mid $\begin{array}{lll}\mathrm{C}(10)-\mathrm{C}(11) & 138,3(3) \\
\mathrm{C}(21)-\mathrm{C}(22) & 152,4(3) \\
\mathrm{C}(21)-\mathrm{C}(23) & 152,4(3) \\
\mathrm{C}(21)-\mathrm{C}(24) & 151,7(3) \\
\mathrm{C}(31)-\mathrm{C}(32) & 154,1(3) \\
\mathrm{C}(31)-\mathrm{C}(33) & 153,7(3) \\
\end{array}$

Fortsetzung der Bindungslängen der Verbindung $\mathbf{Z}$ in pm (mit Standardabweichungen)

\begin{tabular}{|c|c|c|c|}
\hline $\mathrm{C}(6)-\mathrm{B}(1)-\mathrm{C}(1)$ & $109,73(15)$ & $\mathrm{C}(53)-\mathrm{C}(5)-\mathrm{B}(1)$ & $110,60(17)$ \\
\hline$C(6)-B(1)-C(5)$ & $119,82(15)$ & $C(11)-C(6)-C(7)$ & $114,55(17)$ \\
\hline$C(1)-B(1)-C(5)$ & $112,02(15)$ & $C(11)-C(6)-B(1)$ & $124,70(16)$ \\
\hline $\mathrm{C}(6)-\mathrm{B}(1)-\mathrm{N}(2)$ & $109,48(14)$ & $C(7)-C(6)-B(1)$ & $120,33(16)$ \\
\hline $\mathrm{C}(1)-\mathrm{B}(1)-\mathrm{N}(2)$ & $94,78(13)$ & $C(8)-C(7)-C(6)$ & $122,79(18)$ \\
\hline $\mathrm{C}(5)-\mathrm{B}(1)-\mathrm{N}(2)$ & $108,08(14)$ & $\mathrm{C}(9)-\mathrm{C}(8)-\mathrm{C}(7)$ & $120,27(19)$ \\
\hline$C(2)-C(1)-B(1)$ & $104,87(15)$ & $C(10)-C(9)-C(8)$ & $118,80(18)$ \\
\hline $\mathrm{C}(2)-\mathrm{N}(1)-\mathrm{N}(2)$ & $109,02(14)$ & $C(9)-C(10)-C(11)$ & $120,27(18)$ \\
\hline$N(1)-C(2)-C(1)$ & $117,65(16)$ & $C(10)-C(11)-C(6)$ & $123,30(18)$ \\
\hline$N(1)-C(2)-C(21)$ & $118,24(17)$ & $C(24)-C(21)-C(2)$ & $111,10(17)$ \\
\hline$C(1)-C(2)-C(21)$ & $124,10(17)$ & $C(24)-C(21)-C(22)$ & $110,9(2)$ \\
\hline $\mathrm{C}(3)-\mathrm{N}(2)-\mathrm{N}(1)$ & $116,17(15)$ & $C(2)-C(21)-C(22)$ & $108,39(17)$ \\
\hline $\mathrm{C}(3)-\mathrm{N}(2)-\mathrm{B}(1)$ & $133,44(15)$ & $C(24)-C(21)-C(23)$ & $109,1(2)$ \\
\hline$N(1)-N(2)-B(1)$ & $110,36(13)$ & $C(2)-C(21)-C(23)$ & $108,85(17)$ \\
\hline$N(2)-C(3)-C(4)$ & $117,63(16)$ & $C(22)-C(21)-C(23)$ & $108,4(2)$ \\
\hline $\mathrm{N}(2)-\mathrm{C}(3)-\mathrm{C}(31)$ & $123,82(16)$ & $C(34)-C(31)-C(3)$ & $112,03(15)$ \\
\hline $\mathrm{C}(4)-\mathrm{C}(3)-\mathrm{C}(31)$ & $118,56(16)$ & $\mathrm{C}(34)-\mathrm{C}(31)-\mathrm{C}(33)$ & $111,41(17)$ \\
\hline$C(52)-C(5)-C(51)$ & $106,26(17)$ & $C(3)-C(31)-C(33)$ & $108,63(16)$ \\
\hline$C(52)-C(5)-C(53)$ & $107,20(17)$ & $\mathrm{C}(34)-\mathrm{C}(31)-\mathrm{C}(32)$ & $106,40(17)$ \\
\hline$C(51)-C(5)-C(53)$ & $106,22(17)$ & $C(3)-C(31)-C(32)$ & $110,96(16)$ \\
\hline $\mathrm{C}(52)-\mathrm{C}(5)-\mathrm{B}(1)$ & $115,05(15)$ & $\mathrm{C}(33)-\mathrm{C}(31)-\mathrm{C}(32)$ & $107,32(17)$ \\
\hline$C(51)-C(5)-B(1)$ & $111,04(15)$ & & \\
\hline
\end{tabular}

Tabelle 19: Bindungswinkel der Verbindung $\underline{7}$ in ${ }^{\circ}$ (mit Standardabweichung) 


\section{Verbindung 14}

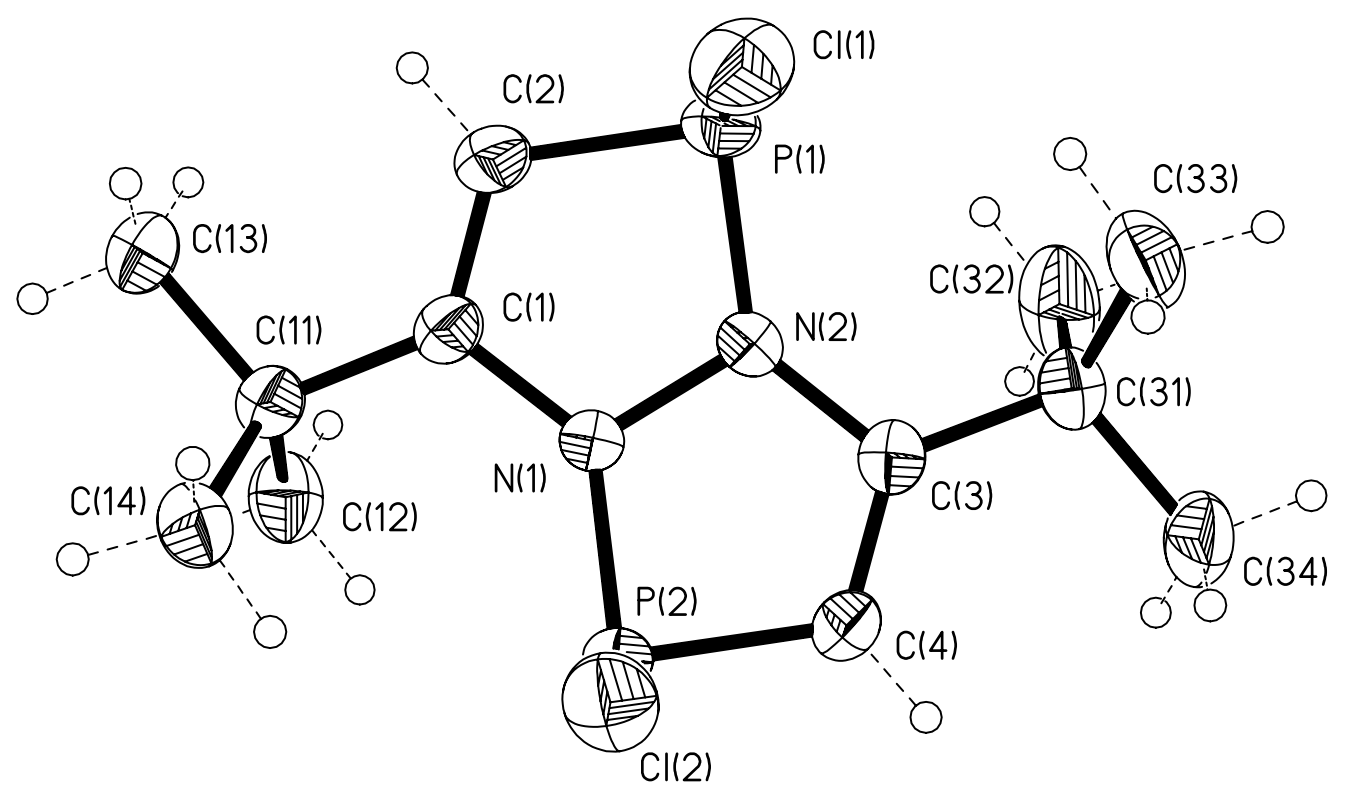

Abbildung 38: Röntgenstruktur von Verbindung $\underline{14}$ mit anisotropen Auslenkungsparametern (50 \%)

$\begin{array}{ll}\mathrm{P}(1)-\mathrm{N}(2) & 169,8(2) \\ \mathrm{P}(1)-\mathrm{C}(2) & 177,1(3) \\ \mathrm{P}(1)-\mathrm{Cl}(1) & 213,8(1) \\ \mathrm{P}(2)-\mathrm{N}(1) & 170,1(2) \\ \mathrm{P}(2)-\mathrm{C}(4) & 177,9(3) \\ \mathrm{P}(2)-\mathrm{Cl}(2) & 215,8(1) \\ \mathrm{N}(1)-\mathrm{C}(1) & 139,9(3) \\ \mathrm{N}(1)-\mathrm{N}(2) & 142,6(3) \\ \mathrm{N}(2)-\mathrm{C}(3) & 139,9(3) \\ \mathrm{C}(1)-\mathrm{C}(2) & 135,3(4)\end{array}$

$\begin{array}{ll}\text { C(1)-C(11) } & 151,9(3) \\ \text { C(3)-C(4) } & 134,1(4) \\ \text { C(3)-C(31) } & 152,3(3) \\ \text { C(11)-C(14) } & 153,5(4) \\ \text { C(11)-C(12) } & 153,5(4) \\ \text { C(11)-C(13) } & 153,8(3) \\ \text { C(31)-C(33) } & 152,5(4) \\ \text { C(31)-C(34) } & 153,2(4) \\ \text { C(31)-C(32) } & 153,6(4)\end{array}$

Tabelle 20: Bindungslängen der Verbindung $\underline{14}$ in pm (mit Standardabweichungen) 


\begin{tabular}{|ll|l|l|}
$\mathrm{N}(2)-\mathrm{P}(1)-\mathrm{C}(2)$ & $88,92(11)$ & $\mathrm{N}(2)-\mathrm{N}(1)-\mathrm{P}(2)$ & $113,94(15)$ \\
$\mathrm{N}(2)-\mathrm{P}(1)-\mathrm{Cl}(1)$ & $105,56(8)$ & $\mathrm{C}(3)-\mathrm{N}(2)-\mathrm{N}(1)$ & $109,94(19)$ \\
$\mathrm{C}(2)-\mathrm{P}(1)-\mathrm{Cl}(1)$ & $100,02(10)$ & $\mathrm{C}(3)-\mathrm{N}(2)-\mathrm{P}(1)$ & $135,67(17)$ \\
$\mathrm{N}(1)-\mathrm{P}(2)-\mathrm{C}(4)$ & $87,77(11)$ & $\mathrm{N}(1)-\mathrm{N}(2)-\mathrm{P}(1)$ & $113,22(15)$ \\
$\mathrm{N}(1)-\mathrm{P}(2)-\mathrm{Cl}(2)$ & $105,20(8)$ & $\mathrm{C}(2)-\mathrm{C}(1)-\mathrm{N}(1)$ & $112,7(2)$ \\
$\mathrm{C}(4)-\mathrm{P}(2)-\mathrm{Cl}(2)$ & $98,95(9)$ & $\mathrm{C}(2)-\mathrm{C}(1)-\mathrm{C}(11)$ & $127,0(2)$ \\
$\mathrm{C}(1)-\mathrm{N}(1)-\mathrm{N}(2)$ & $110,66(18)$ & $\mathrm{N}(1)-\mathrm{C}(1)-\mathrm{C}(11)$ & $120,1(2)$ \\
$\mathrm{C}(1)-\mathrm{N}(1)-\mathrm{P}(2)$ & $134,03(17)$ & $\mathrm{C}(1)-\mathrm{C}(2)-\mathrm{P}(1)$ & $113,18(19)$
\end{tabular}

Tabelle 21: Bindungswinkel der Verbindung $\underline{14}$ in ${ }^{\circ}$ (mit Standardabweichung) 


\section{Verbindung $\underline{15}$}

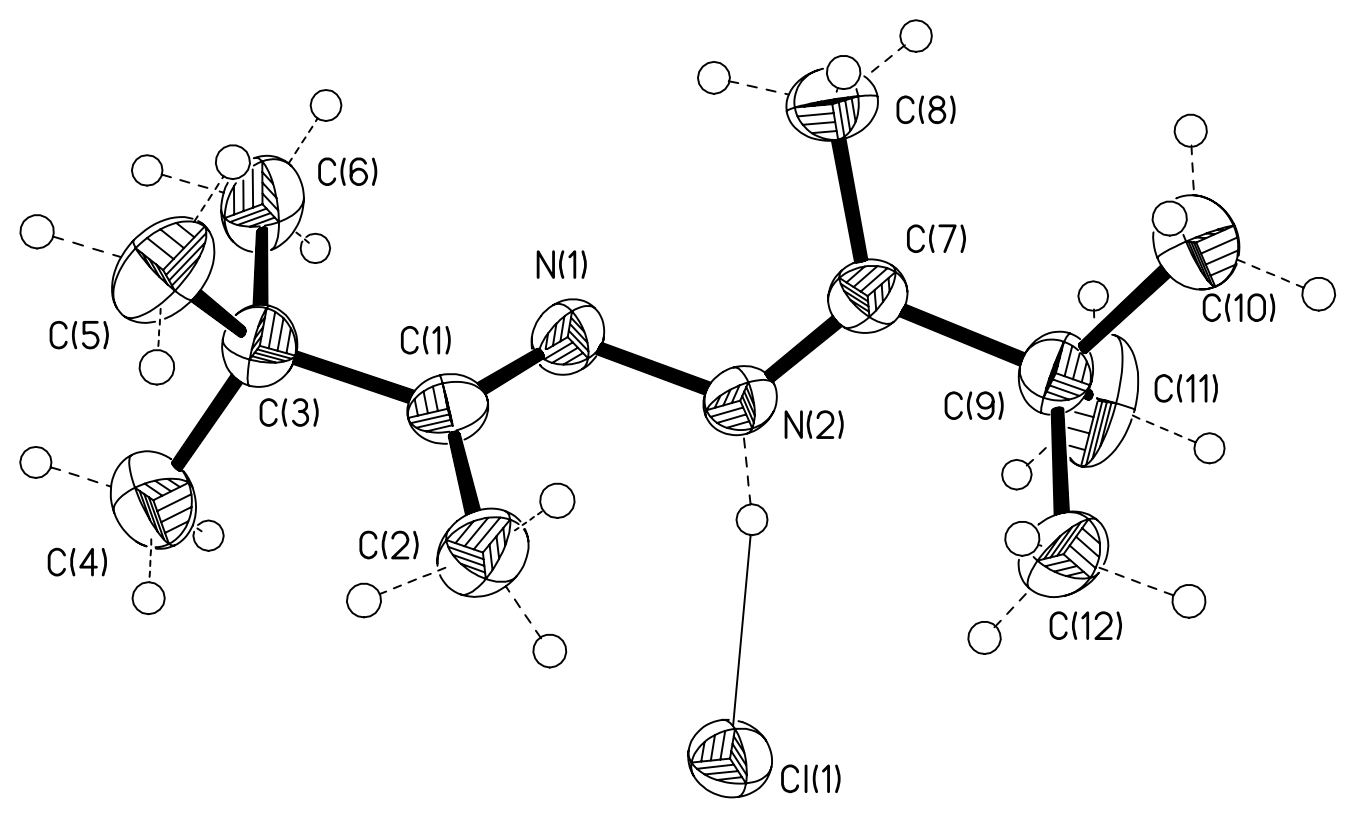

Abbildung 39: Röntgenstruktur von Verbindung $\underline{15}$ mit anisotropen Auslenkungsparametern (50 \%)

\begin{tabular}{ll|l|ll}
$\mathrm{N}(1)-\mathrm{C}(1)$ & $126,9(4)$ & $\mathrm{C}(3)-\mathrm{C}(5)$ & $153,6(4)$ \\
$\mathrm{N}(1)-\mathrm{N}(2)$ & $142,3(3)$ & $\mathrm{C}(7)-\mathrm{C}(8)$ & $147,7(4)$ \\
$\mathrm{N}(2)-\mathrm{C}(7)$ & $128,2(4)$ & $\mathrm{C}(7)-\mathrm{C}(9)$ & $152,3(4)$ \\
$\mathrm{C}(1)-\mathrm{C}(2)$ & $149,5(4)$ & & $\mathrm{C}(9)-\mathrm{C}(11)$ & $152,4(5)$ \\
$\mathrm{C}(1)-\mathrm{C}(3)$ & $151,2(4)$ & $\mathrm{C}(9)-\mathrm{C}(10)$ & $152,7(4)$ \\
$\mathrm{C}(3)-\mathrm{C}(6)$ & $152,6(4)$ & $\mathrm{C}(9)-\mathrm{C}(12)$ & $153,6(4)$ \\
$\mathrm{C}(3)-\mathrm{C}(4)$ & $153,5(4)$ &
\end{tabular}

Tabelle 22: Bindungslängen der Verbindung $\underline{15}$ in pm (mit Standardabweichungen) 


\begin{tabular}{|c|c|c|c|}
\hline $\mathrm{C}(1)-\mathrm{N}(1)-\mathrm{N}(2)$ & $114,1(2)$ & $C(4)-C(3)-C(5)$ & $109,2(3)$ \\
\hline $\mathrm{C}(7)-\mathrm{N}(2)-\mathrm{N}(1)$ & $121,2(2)$ & $N(2)-C(7)-C(8)$ & $120,1(3)$ \\
\hline$N(1)-C(1)-C(2)$ & $123,6(3)$ & $N(2)-C(7)-C(9)$ & $117,9(2)$ \\
\hline$N(1)-C(1)-C(3)$ & $116,7(2)$ & $\mathrm{C}(8)-\mathrm{C}(7)-\mathrm{C}(9)$ & $122,1(3)$ \\
\hline$C(2)-C(1)-C(3)$ & $119,7(2)$ & $C(7)-C(9)-C(11)$ & $107,2(3)$ \\
\hline$C(1)-C(3)-C(6)$ & $111,8(2)$ & $C(7)-C(9)-C(10)$ & $110,9(3)$ \\
\hline$C(1)-C(3)-C(4)$ & $109,3(2)$ & $C(11)-C(9)-C(10)$ & $110,4(3)$ \\
\hline$C(6)-C(3)-C(4)$ & $109,0(3)$ & $C(7)-C(9)-C(12)$ & $109,7(2)$ \\
\hline$C(1)-C(3)-C(5)$ & $109,1(2)$ & $C(11)-C(9)-C(12)$ & $111,6(3)$ \\
\hline$C(6)-C(3)-C(5)$ & $108,4(3)$ & $C(10)-C(9)-C(12)$ & $107,1(3)$ \\
\hline
\end{tabular}

Tabelle 23: Bindungswinkel der Verbindung $\underline{15}$ in ${ }^{\circ}$ (mit Standardabweichung) 


\section{Verbindung 17}

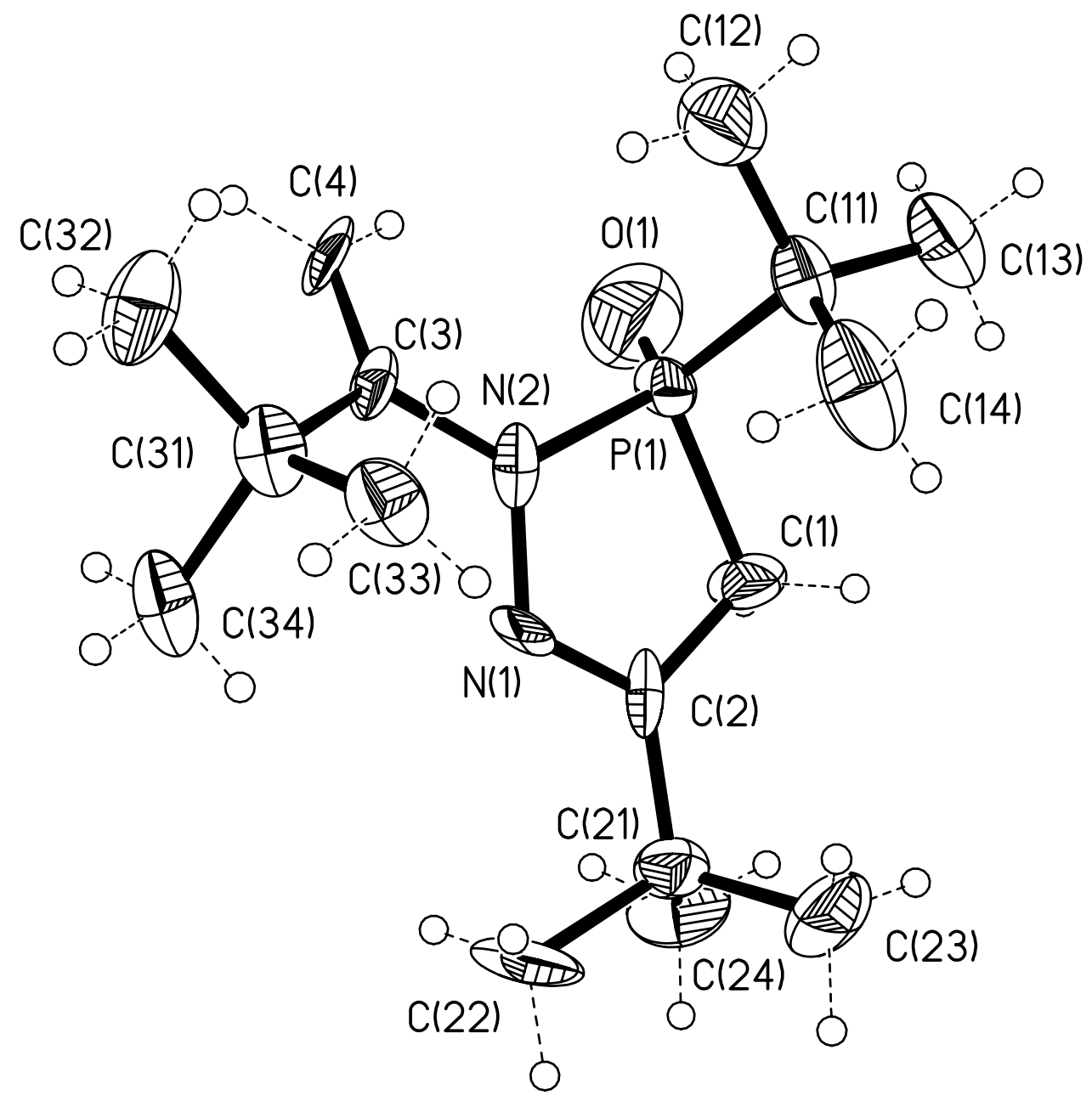

Abbildung 40: Röntgenstruktur von Verbindung $\underline{17}$ mit anisotropen Auslenkungsparametern (50\%)

\begin{tabular}{|cc|c|c|}
$\mathrm{P}(1)-\mathrm{O}(1)$ & $138,3(12)$ & $\mathrm{N}(1)-\mathrm{C}(1)$ & $140,3(10)$ \\
$\mathrm{P}(1)-\mathrm{N}(1)$ & $171,7(7)$ & $\mathrm{C}(1)-\mathrm{C}(2)$ & $133,6(14)$ \\
$\mathrm{P}(1)-\mathrm{C}(21)$ & $180,9(10)$ & $\mathrm{C}(1)-\mathrm{C}(11)$ & $154,7(13)$ \\
$\mathrm{P}(1)-\mathrm{C}(4)$ & $183,9(9)$ & $\mathrm{N}(2)-\mathrm{C}(3)$ & $130,8(11)$ \\
$\mathrm{N}(1)-\mathrm{N}(2)$ & $137,0(10)$ & $\mathrm{C}(3)-\mathrm{C}(4)$ & $148,3(13)$
\end{tabular}

Tabelle 24: Bindungslängen der Verbindung $\underline{17}$ in pm (mit Standardabweichungen) 


\begin{tabular}{cc|}
$\mathrm{C}(3)-\mathrm{C}(31)$ & $149,6(13)$ \\
$\mathrm{C}(11)-\mathrm{C}(13)$ & $154,8(14)$ \\
$\mathrm{C}(11)-\mathrm{C}(14)$ & $155,0(14)$ \\
$\mathrm{C}(11)-\mathrm{C}(12)$ & $156,2(14)$ \\
$\mathrm{C}(21)-\mathrm{C}(23)$ & $150,9(14)$
\end{tabular} \mid $\begin{array}{lll}\mathrm{C}(21)-\mathrm{C}(22) & 152,3(15) \\
\mathrm{C}(21)-\mathrm{C}(24) & 156,7(15) \\
\mathrm{C}(31)-\mathrm{C}(33) & 153,0(15) \\
\mathrm{C}(31)-\mathrm{C}(32) & 154,6(14) \\
\mathrm{C}(31)-\mathrm{C}(34) & 155,6(16)\end{array}$

Fortsetzung der Bindungslängen der Verbindung $\underline{17}$ in pm (mit Standardabweichungen)

\begin{tabular}{|c|c|c|c|}
\hline $\mathrm{O}(1)-\mathrm{P}(1)-\mathrm{N}(1)$ & $121,1(5)$ & $C(13)-C(11)-C(1)$ & $112,2(7)$ \\
\hline $\mathrm{O}(1)-\mathrm{P}(1)-\mathrm{C}(21)$ & $116,0(6)$ & $C(14)-C(11)-C(1)$ & $110,3(9)$ \\
\hline$N(1)-P(1)-C(21)$ & $106,9(4)$ & $C(13)-C(11)-C(12)$ & $106,6(9)$ \\
\hline $\mathrm{O}(1)-\mathrm{P}(1)-\mathrm{C}(4)$ & $112,1(7)$ & $C(14)-C(11)-C(12)$ & $107,8(9)$ \\
\hline$N(1)-P(1)-C(4)$ & $90,5(4)$ & $C(1)-C(11)-C(12)$ & $109,6(8)$ \\
\hline$C(21)-P(1)-C(4)$ & $106,7(6)$ & $C(23)-C(21)-C(22)$ & $110,4(9)$ \\
\hline$N(2)-N(1)-C(1)$ & $119,5(7)$ & $C(23)-C(21)-C(24)$ & $109,4(9)$ \\
\hline$N(2)-N(1)-P(1)$ & $114,0(6)$ & $C(22)-C(21)-C(24)$ & $107,9(10)$ \\
\hline$C(1)-N(1)-P(1)$ & $125,2(7)$ & $C(23)-C(21)-P(1)$ & $107,8(8)$ \\
\hline$C(2)-C(1)-N(1)$ & $120,3(8)$ & $C(22)-C(21)-P(1)$ & $109,2(7)$ \\
\hline$C(2)-C(1)-C(11)$ & $122,6(8)$ & $C(24)-C(21)-P(1)$ & $112,1(7)$ \\
\hline$N(1)-C(1)-C(11)$ & $117,1(8)$ & $C(3)-C(31)-C(33)$ & $108,5(9)$ \\
\hline$C(3)-N(2)-N(1)$ & $114,8(7)$ & $C(3)-C(31)-C(32)$ & $109,7(8)$ \\
\hline$N(2)-C(3)-C(4)$ & $115,7(8)$ & $C(33)-C(31)-C(32)$ & $110,1(10)$ \\
\hline$N(2)-C(3)-C(31)$ & $122,4(8)$ & $C(3)-C(31)-C(34)$ & $109,2(9)$ \\
\hline$C(4)-C(3)-C(31)$ & $121,8(8)$ & $C(33)-C(31)-C(34)$ & $112,0(10)$ \\
\hline$C(3)-C(4)-P(1)$ & $105,0(6)$ & $\mathrm{C}(32)-\mathrm{C}(31)-\mathrm{C}(34)$ & $107,2(10)$ \\
\hline$C(13)-C(11)-C(14)$ & $110,2(8)$ & & \\
\hline
\end{tabular}

Tabelle 25: Bindungswinkel der Verbindung $\underline{17}$ in ${ }^{\circ}$ (mit Standardabweichung) 


\section{Verbindung 21}

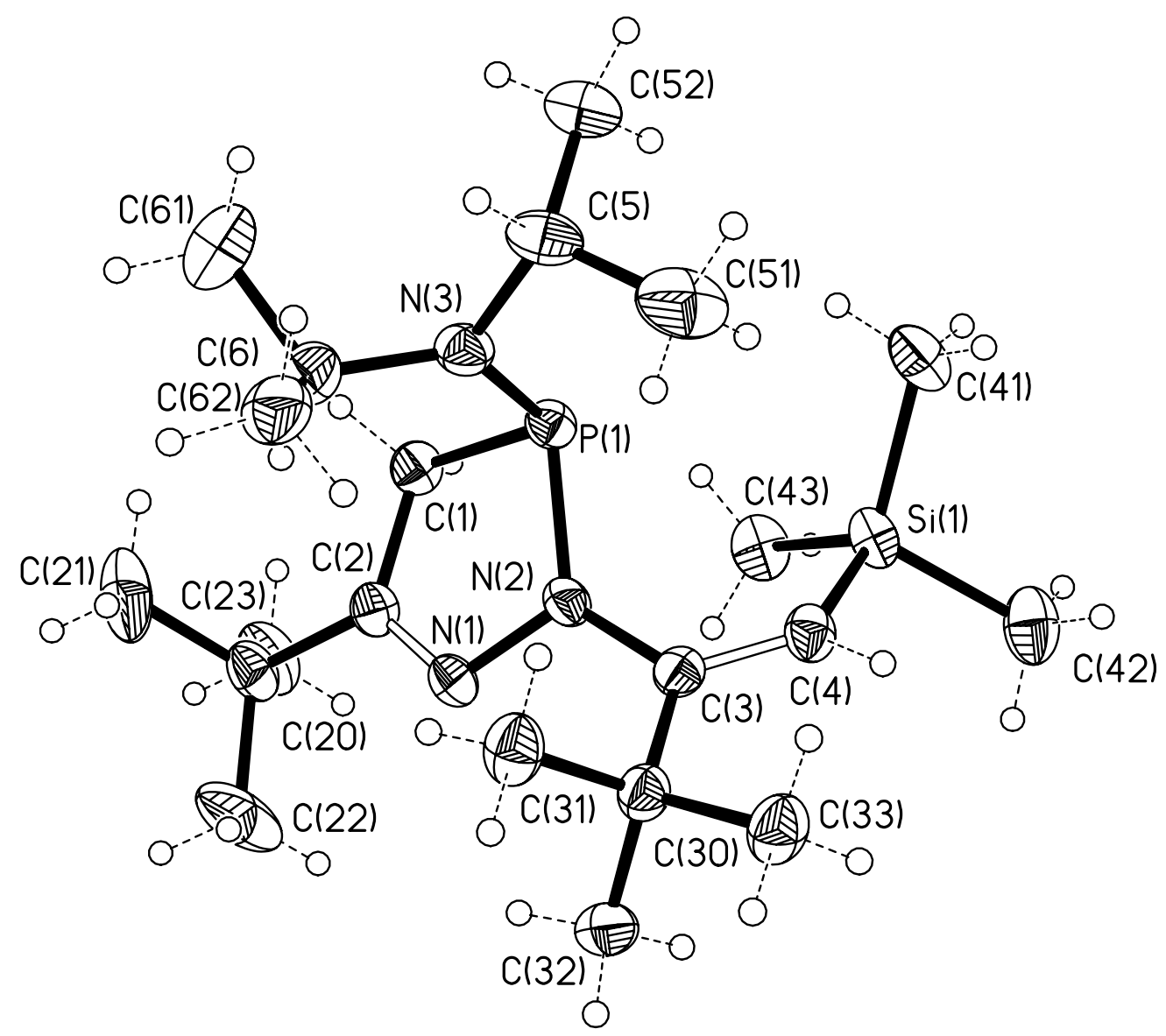

Abbildung 41: Röntgenstruktur von Verbindung $\underline{21}$ mit anisotropen Auslenkungsparametern (50 \%)

$\begin{array}{ll}\mathrm{P}(1)-\mathrm{N}(3) & 168,2(1) \\ \mathrm{P}(1)-\mathrm{N}(2) & 175,1(1) \\ \mathrm{P}(1)-\mathrm{C}(1) & 184,6(2) \\ \mathrm{Si}(1)-\mathrm{C}(4) & 186,1(2) \\ \mathrm{Si}(1)-\mathrm{C}(41) & 186,8(2) \\ \mathrm{Si}(1)-\mathrm{C}(42) & 187,6(2)\end{array}$

$C(3)-C(30)$
$N(3)-C(6)$
$N(3)-C(5)$
$C(5)-C(52)$
$C(5)-C(51)$
$C(6)-C(62)$

$154,1(2)$ $148,0(2)$ $149,3(2)$ $151,9(2)$ $152,0(3)$ $152,4(3)$

Tabelle 26: Bindungslängen der Verbindung $\underline{21}$ in pm (mit Standardabweichungen) 


\begin{tabular}{|c|c|c|c|}
\hline $\mathrm{Si}(1)-\mathrm{C}(43)$ & $187,9(2)$ & $C(6)-C(61)$ & $153,1(3)$ \\
\hline$N(1)-C(2)$ & $127,7(2)$ & $C(20)-C(22)$ & $151,7(3)$ \\
\hline $\mathrm{N}(1)-\mathrm{N}(2)$ & $141,4(2)$ & $C(20)-C(23)$ & $152,3(2)$ \\
\hline$C(1)-C(2)$ & $151,4(2)$ & $C(20)-C(21)$ & $153,2(3)$ \\
\hline$N(2)-C(3)$ & $141,6(2)$ & $C(30)-C(31)$ & $152,8(2)$ \\
\hline$C(2)-C(20)$ & $152,2(2)$ & $C(30)-C(33)$ & $153,8(2)$ \\
\hline$C(3)-C(4)$ & $134,9(2)$ & $C(30)-C(32)$ & $154,8(2)$ \\
\hline
\end{tabular}

Fortsetzung der Bindungslängen der Verbindung $\underline{21}$ in pm (mit Standardabweichungen)

\begin{tabular}{|c|c|c|c|}
\hline$N(3)-P(1)-N(2)$ & $109,35(7)$ & $C(6)-N(3)-P(1)$ & $126,47(11)$ \\
\hline$N(3)-P(1)-C(1)$ & $103,58(8)$ & $C(5)-N(3)-P(1)$ & $116,88(12)$ \\
\hline$N(2)-P(1)-C(1)$ & $86,09(7)$ & $C(3)-C(4)-S i(1)$ & $132,50(13)$ \\
\hline$C(4)-S i(1)-C(41)$ & $114,76(8)$ & $N(3)-C(5)-C(52)$ & $112,86(14)$ \\
\hline$C(4)-S i(1)-C(42)$ & $104,15(9)$ & $N(3)-C(5)-C(51)$ & $111,33(14)$ \\
\hline$C(41)-S i(1)-C(42)$ & $106,74(10)$ & $C(52)-C(5)-C(51)$ & $111,32(17)$ \\
\hline$C(4)-S i(1)-C(43)$ & $113,08(8)$ & $N(3)-C(6)-C(62)$ & $114,29(15)$ \\
\hline$C(41)-S i(1)-C(43)$ & $109,26(9)$ & $N(3)-C(6)-C(61)$ & $111,42(16)$ \\
\hline$C(42)-S i(1)-C(43)$ & $108,36(9)$ & $C(62)-C(6)-C(61)$ & $109,97(16)$ \\
\hline $\mathrm{C}(2)-\mathrm{N}(1)-\mathrm{N}(2)$ & $110,94(13)$ & $C(22)-C(20)-C(2)$ & $110,41(15)$ \\
\hline$C(2)-C(1)-P(1)$ & $105,90(11)$ & $C(22)-C(20)-C(23)$ & $109,86(17)$ \\
\hline $\mathrm{N}(1)-\mathrm{N}(2)-\mathrm{C}(3)$ & $117,86(12)$ & $C(2)-C(20)-C(23)$ & $108,82(14)$ \\
\hline$N(1)-N(2)-P(1)$ & $116,05(10)$ & $C(22)-C(20)-C(21)$ & $109,86(19)$ \\
\hline $\mathrm{C}(3)-\mathrm{N}(2)-\mathrm{P}(1)$ & $125,62(10)$ & $C(2)-C(20)-C(21)$ & $109,72(15)$ \\
\hline$N(1)-C(2)-C(1)$ & $116,41(13)$ & $C(23)-C(20)-C(21)$ & $108,13(17)$ \\
\hline $\mathrm{N}(1)-\mathrm{C}(2)-\mathrm{C}(20)$ & $120,41(15)$ & $C(31)-C(30)-C(33)$ & $107,04(14)$ \\
\hline$C(1)-C(2)-C(20)$ & $123,14(14)$ & $C(31)-C(30)-C(3)$ & $110,84(14)$ \\
\hline $\mathrm{C}(4)-\mathrm{C}(3)-\mathrm{N}(2)$ & $118,86(15)$ & $C(33)-C(30)-C(3)$ & $111,08(13)$ \\
\hline$C(4)-C(3)-C(30)$ & $123,63(15)$ & $C(31)-C(30)-C(32)$ & $109,94(14)$ \\
\hline$N(2)-C(3)-C(30)$ & $117,50(13)$ & $C(33)-C(30)-C(32)$ & $107,81(14)$ \\
\hline$C(6)-N(3)-C(5)$ & $116,58(14)$ & $C(3)-C(30)-C(32)$ & $110,05(13)$ \\
\hline
\end{tabular}

Tabelle 27: Bindungswinkel der Verbindung 21 in ${ }^{\circ}$ (mit Standardabweichung) 


\section{Verbindung 25}

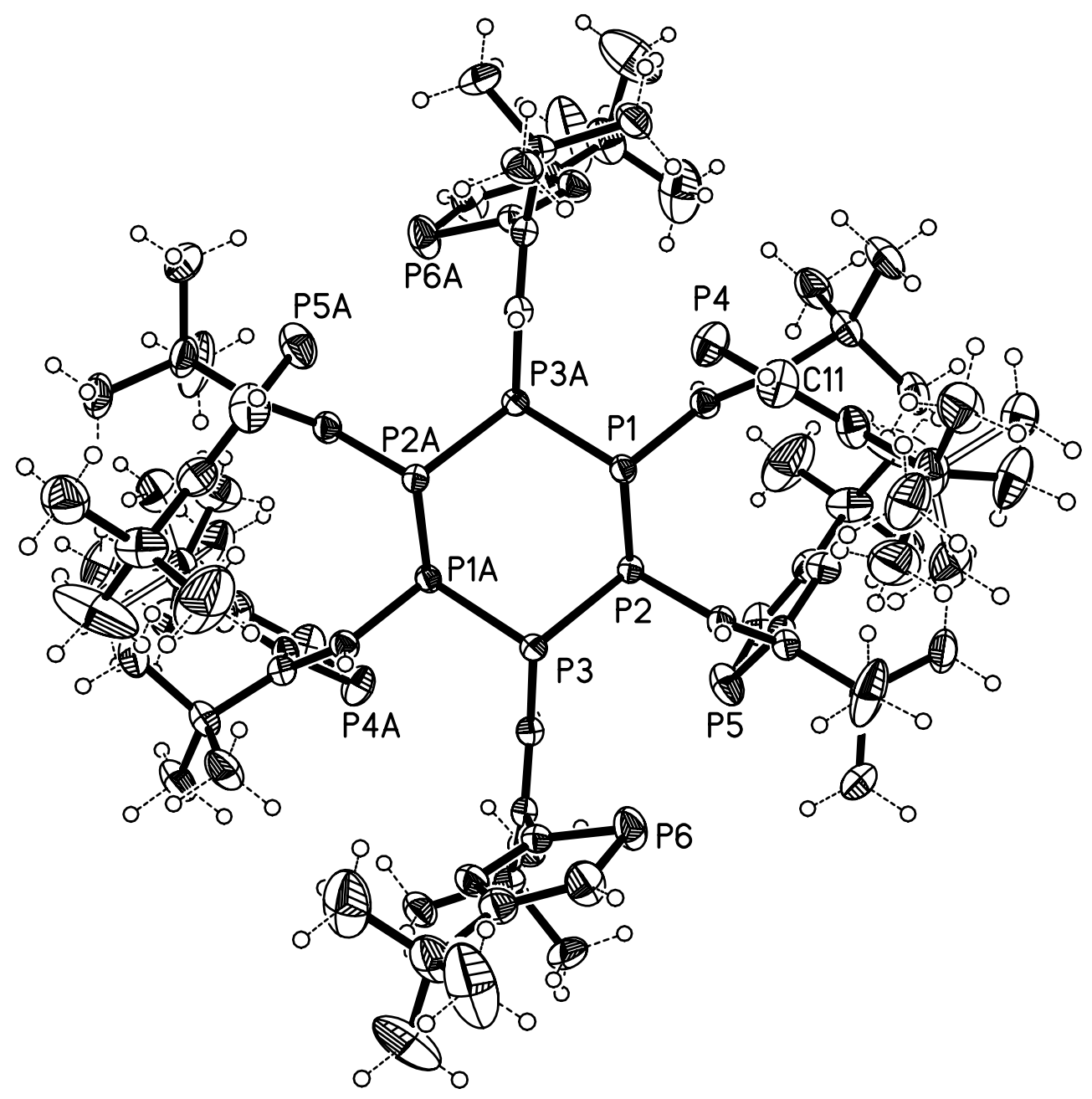

Abbildung 42: Röntgenstruktur von Verbindung $\underline{25}$ mit anisotropen Auslenkungsparametern (50 \%)

$\begin{array}{cc}P(1)-C(14) & 182,1(2) \\ P(1)-P(3) \# 1 & 223,60(8) \\ P(1)-P(2) & 224,68(10) \\ P(2)-C(24) & 182,5(2) \\ P(2)-P(3) & 223,86(8)\end{array}$

$P(3)-C(34)$
$P(3)-P(1) \# 1$
$P(4)-N(11)$
$P(4)-C(11)$
$P(5)-N(21)$

$182,7(2)$ $223,60(8)$ $168,76(18)$ $169,8(2)$ $168,60(18)$

Tabelle 28: Bindungslängen der Verbindung $\underline{25}$ in pm (mit Standardabweichungen) 


\begin{tabular}{|c|c|c|c|}
\hline$P(5)-C(21)$ & $169,6(3)$ & $C(12)-C(120)$ & $152,1(3)$ \\
\hline$P(6)-N(31)$ & $168,30(17)$ & $C(120)-C(122)$ & $153,2(2)$ \\
\hline$P(6)-C(31)$ & $170,2(3)$ & $C(120)-C(125)$ & $153,3(2)$ \\
\hline $\mathrm{N}(11)-\mathrm{N}(12)$ & $136,1(2)$ & $C(120)-C(123)$ & $153,3(2)$ \\
\hline$N(11)-C(13)$ & $143,9(3)$ & $C(120)-C(124)$ & $153,4(2)$ \\
\hline$C(11)-C(12)$ & $139,9(3)$ & $C(120)-C(126)$ & $153,6(2)$ \\
\hline $\mathrm{N}(12)-\mathrm{C}(12)$ & $133,2(3)$ & $C(120)-C(121)$ & $153,6(2)$ \\
\hline$C(13)-C(14)$ & $133,7(3)$ & $C(130)-C(131)$ & $153,4(3)$ \\
\hline$C(13)-C(130)$ & $153,3(3)$ & $C(130)-C(132)$ & $153,6(3)$ \\
\hline$C(21)-C(22)$ & $140,2(3)$ & $C(130)-C(133)$ & $153,8(3)$ \\
\hline$N(21)-N(22)$ & $136,3(2)$ & $C(220)-C(221)$ & $152,0(4)$ \\
\hline $\mathrm{N}(21)-\mathrm{C}(23)$ & $144,8(3)$ & $C(220)-C(222)$ & $152,4(4)$ \\
\hline$N(22)-C(22)$ & $133,4(3)$ & $C(220)-C(223)$ & $152,7(4)$ \\
\hline$C(22)-C(220)$ & $152,3(3)$ & $C(230)-C(231)$ & $152,9(4)$ \\
\hline$C(23)-C(24)$ & $133,2(3)$ & $C(230)-C(232)$ & $153,0(3)$ \\
\hline$C(23)-C(230)$ & $152,8(3)$ & $C(230)-C(233)$ & $154,3(4)$ \\
\hline $\mathrm{N}(31)-\mathrm{N}(32)$ & $135,6(2)$ & $C(320)-C(322)$ & $150,3(4)$ \\
\hline $\mathrm{N}(31)-\mathrm{C}(33)$ & $144,2(3)$ & $C(320)-C(321)$ & $152,6(4)$ \\
\hline$C(31)-C(32)$ & $139,5(3)$ & $C(320)-C(323)$ & $154,5(5)$ \\
\hline $\mathrm{N}(32)-\mathrm{C}(32)$ & $132,6(3)$ & $C(330)-C(331)$ & $153,1(3)$ \\
\hline$C(32)-C(320)$ & $152,5(3)$ & $C(330)-C(332)$ & $153,4(3)$ \\
\hline$C(33)-C(34)$ & $133,8(3)$ & $C(330)-C(333)$ & $154,1(3)$ \\
\hline$C(33)-C(330)$ & $152,9(3)$ & & \\
\hline
\end{tabular}

Fortsetzung der Bindungslängen der Verbindung $\underline{25}$ in pm (mit Standardabweichungen)

\begin{tabular}{|cc|c|cc|}
$\mathrm{C}(14)-\mathrm{P}(1)-\mathrm{P}(3) \# 1$ & $96,36(7)$ & $\mathrm{P}(1) \# 1-\mathrm{P}(3)-\mathrm{P}(2)$ & $93,97(3)$ \\
$\mathrm{C}(14)-\mathrm{P}(1)-\mathrm{P}(2)$ & $95,82(7)$ & $\mathrm{N}(11)-\mathrm{P}(4)-\mathrm{C}(11)$ & $88,80(10)$ \\
$\mathrm{P}(3) \# 1-\mathrm{P}(1)-\mathrm{P}(2)$ & $95,29(3)$ & $\mathrm{N}(21)-\mathrm{P}(5)-\mathrm{C}(21)$ & $88,87(10)$ \\
$\mathrm{C}(24)-\mathrm{P}(2)-\mathrm{P}(3)$ & $96,17(7)$ & $\mathrm{N}(31)-\mathrm{P}(6)-\mathrm{C}(31)$ & $88,34(10)$ \\
$\mathrm{C}(24)-\mathrm{P}(2)-\mathrm{P}(1)$ & $94,56(7)$ & $\mathrm{N}(12)-\mathrm{N}(11)-\mathrm{C}(13)$ & $118,78(16)$ \\
$\mathrm{P}(3)-\mathrm{P}(2)-\mathrm{P}(1)$ & $91,33(4)$ & $\mathrm{N}(12)-\mathrm{N}(11)-\mathrm{P}(4)$ & $116,52(14)$ \\
$\mathrm{C}(34)-\mathrm{P}(3)-\mathrm{P}(1) \# 1$ & $93,76(6)$ & $\mathrm{C}(13)-\mathrm{N}(11)-\mathrm{P}(4)$ & $124,62(13)$ \\
$\mathrm{C}(34)-\mathrm{P}(3)-\mathrm{P}(2)$ & $98,61(7)$ & $\mathrm{C}(12)-\mathrm{C}(11)-\mathrm{P}(4)$ & $110,55(17)$
\end{tabular}

Tabelle 29: Bindungswinkel der Verbindung $\underline{25}$ in ${ }^{\circ}$ (mit Standardabweichung) 


\begin{tabular}{|c|c|c|c|}
\hline $\mathrm{C}(12)-\mathrm{N}(12)-\mathrm{N}(11)$ & $108,60(17)$ & $C(12)-C(120)-C(125)$ & $110,6(3)$ \\
\hline $\mathrm{C}(14)-\mathrm{C}(13)-\mathrm{N}(11)$ & $119,44(17)$ & $C(12)-C(120)-C(126)$ & $108,8(3)$ \\
\hline$C(14)-C(13)-C(130)$ & $124,14(18)$ & $C(122)-C(120)-C(126)$ & $59,2(3)$ \\
\hline$N(11)-C(13)-C(130)$ & $116,41(16)$ & $C(125)-C(120)-C(126)$ & $105,4(4)$ \\
\hline$C(13)-C(14)-P(1)$ & $124,15(16)$ & $C(123)-C(120)-C(126)$ & $62,4(3)$ \\
\hline$C(22)-C(21)-P(5)$ & $110,85(17)$ & $C(124)-C(120)-C(126)$ & $102,3(4)$ \\
\hline $\mathrm{N}(22)-\mathrm{N}(21)-\mathrm{C}(23)$ & $117,77(16)$ & $C(12)-C(120)-C(121)$ & $107,6(2)$ \\
\hline$N(22)-N(21)-P(5)$ & $116,40(14)$ & $C(122)-C(120)-C(121)$ & $108,0(3)$ \\
\hline$C(23)-N(21)-P(5)$ & $125,76(14)$ & $C(125)-C(120)-C(121)$ & $61,8(3)$ \\
\hline $\mathrm{C}(22)-\mathrm{N}(22)-\mathrm{N}(21)$ & $108,89(17)$ & $C(123)-C(120)-C(121)$ & $106,1(3)$ \\
\hline$N(22)-C(22)-C(21)$ & $115,0(2)$ & $C(124)-C(120)-C(121)$ & $60,9(3)$ \\
\hline$N(22)-C(22)-C(220)$ & $120,1(2)$ & $C(126)-C(120)-C(121)$ & $143,6(3)$ \\
\hline$C(21)-C(22)-C(220)$ & $124,9(2)$ & $C(13)-C(130)-C(131)$ & $110,27(17)$ \\
\hline$C(24)-C(23)-N(21)$ & $118,88(17)$ & $C(13)-C(130)-C(132)$ & $108,48(17)$ \\
\hline$C(24)-C(23)-C(230)$ & $125,16(19)$ & $C(131)-C(130)-C(132)$ & $110,36(18)$ \\
\hline$N(21)-C(23)-C(230)$ & $115,81(17)$ & $C(13)-C(130)-C(133)$ & $110,27(17)$ \\
\hline$C(23)-C(24)-P(2)$ & $123,68(16)$ & $C(131)-C(130)-C(133)$ & $108,23(19)$ \\
\hline $\mathrm{N}(32)-\mathrm{N}(31)-\mathrm{C}(33)$ & $118,89(16)$ & $C(132)-C(130)-C(133)$ & $109,22(19)$ \\
\hline$N(32)-N(31)-P(6)$ & $116,74(14)$ & $C(221)-C(220)-C(22)$ & $111,2(2)$ \\
\hline $\mathrm{C}(33)-\mathrm{N}(31)-\mathrm{P}(6)$ & $124,24(13)$ & $C(221)-C(220)-C(222)$ & $111,6(3)$ \\
\hline$C(32)-C(31)-P(6)$ & $110,86(17)$ & $C(22)-C(220)-C(222)$ & $109,3(2)$ \\
\hline $\mathrm{C}(32)-\mathrm{N}(32)-\mathrm{N}(31)$ & $108,99(17)$ & $C(221)-C(220)-C(223)$ & $107,6(3)$ \\
\hline $\mathrm{N}(32)-\mathrm{C}(32)-\mathrm{C}(31)$ & $115,1(2)$ & $C(22)-C(220)-C(223)$ & $109,2(2)$ \\
\hline$N(32)-C(32)-C(320)$ & $117,7(2)$ & $C(222)-C(220)-C(223)$ & $107,8(2)$ \\
\hline$C(31)-C(32)-C(320)$ & $127,2(2)$ & $C(23)-C(230)-C(231)$ & $110,57(19)$ \\
\hline $\mathrm{C}(34)-\mathrm{C}(33)-\mathrm{N}(31)$ & $118,39(18)$ & $C(23)-C(230)-C(232)$ & $111,03(18)$ \\
\hline$C(34)-C(33)-C(330)$ & $125,50(19)$ & $C(231)-C(230)-C(232)$ & $108,6(2)$ \\
\hline$N(31)-C(33)-C(330)$ & $116,06(17)$ & $C(23)-C(230)-C(233)$ & $108,07(19)$ \\
\hline$C(33)-C(34)-P(3)$ & $123,07(16)$ & $C(231)-C(230)-C(233)$ & $109,7(2)$ \\
\hline$N(12)-C(12)-C(11)$ & $115,54(19)$ & $C(232)-C(230)-C(233)$ & $108,9(2)$ \\
\hline$N(12)-C(12)-C(120)$ & $119,12(19)$ & $C(322)-C(320)-C(32)$ & $111,8(2)$ \\
\hline$C(11)-C(12)-C(120)$ & $125,35(19)$ & $C(322)-C(320)-C(321)$ & $110,2(3)$ \\
\hline$C(12)-C(120)-C(122)$ & $107,8(2)$ & $C(32)-C(320)-C(321)$ & $109,4(2)$ \\
\hline
\end{tabular}

Fortsetzung der Bindungswinkel der Verbindung $\underline{\mathbf{2 5}}$ in ${ }^{\circ}$ (mit Standardabweichung) 


\begin{tabular}{|c|c|c|c|}
\hline$C(322)-C(320)-C(323)$ & $110,3(3)$ & $C(32)-C(320)-C(323)$ & $107,9(2)$ \\
\hline$C(122)-C(120)-C(125)$ & $141,5(3)$ & $C(321)-C(320)-C(323)$ & $107,1(3)$ \\
\hline$C(12)-C(120)-C(123)$ & $109,1(2)$ & $\mathrm{C}(33)-\mathrm{C}(330)-\mathrm{C}(331)$ & $110,49(17)$ \\
\hline$C(122)-C(120)-C(123)$ & $117,8(4)$ & $C(33)-C(330)-C(332)$ & $109,17(18)$ \\
\hline$C(125)-C(120)-C(123)$ & $45,9(3)$ & $C(331)-C(330)-C(332)$ & $108,95(19)$ \\
\hline$C(12)-C(120)-C(124)$ & $114,2(3)$ & $C(33)-C(330)-C(333)$ & $109,66(17)$ \\
\hline$C(122)-C(120)-C(124)$ & $47,7(3)$ & $C(331)-C(330)-C(333)$ & $108,71(18)$ \\
\hline$C(125)-C(120)-C(124)$ & $114,7(4)$ & $C(332)-C(330)-C(333)$ & $109,85(18)$ \\
\hline$C(123)-C(120)-C(124)$ & $136,7(3)$ & & \\
\hline
\end{tabular}

Fortsetzung der Bindungswinkel der Verbindung $\underline{25}$ in ${ }^{\circ}$ (mit Standardabweichung) 


\section{Literaturverzeichnis}

"An expert is someone who has made all the mistakes that can be made, but in a very narrow field." Niels Bohr

1 Houben-Weyl: Methoden der organischen Chemie. Organische Stickstoffverbindungen mit einer C,N-Doppelbindung. Teil 2. Bd. E14b. S. 640. Stuttgart: 1990. Georg Thieme Verlag.

2 Houben-Weyl: Methoden der organischen Chemie. Ketone II. Bd. 7/2b. S. 1954. Stuttgart: 1976. Georg Thieme Verlag.

3 Houben-Weyl: Methoden der organischen Chemie. Stickstoffverbindungen I. Teil 2. Bd. 10/2. S. 89. Stuttgart: 1976. Georg Thieme Verlag.

$4 \quad$ E. Lederer: Bull. Soc. Chim. Fr. 1946, 172.

5 F. Henoch, K. G. Hampton, C.R. Hauser: J. Am. Chem. Soc. 1969, 91, 676.

6 T. Groh, G. Elter, M. Noltemeyer, H.-G. Schmidt, A. Meller: Organomet. 2000, 19, 2477.

7 T. Groh, G. Elter, M. Noltemeyer, H.-G. Schmidt, A. Meller: Main Group Metal Chem. 2000, 23, 709.

8 F. Armbruster: Staatsexamensarbeit. Universität Göttingen 2001.

9 S. Patai: The Chemistry of the Carbon-Nitrogen Double Bond. S. 398. New York: 1970. Interscience.

10 E. Arnal, J. Elguero, R. Jacquier, C. Marzin: Bull. Soc. Chim. Fr. 1965, 877.

11 J. Elguero, R. Jacquier, C. Marzin: Bull. Soc. Chim. Fr. 1968, 713.

12 K. Appenroth, M. Reichenbaecher, R. Paetzold: Tetrahedron 1981, 37, 569.

13 D. S. Malament, J. M. McBride: J. Am. Chem. Soc. 1970, 92, 4586.

14 K. Niedenzu, J. W. Dawson: Boron-Nitrogen Compounds. Berlin - Heidelberg New York: 1965. Springer-Verlag.

15 H. Nöth: Angew. Chem. 1988, 100, 1664.

16 P. Paetzold: Adv. Inorg. Chem. 1987, 31, 123.

17 C. Boeker: Main Group Metal Chem. 1998, 21, 565.

18 S. Toyota, M. Oki: Bull. Chem. Soc. Jpn. 1992, 65, 1832. 
19 A. Meller, H. Hoppe, W. Marringele, A. Haase, M. Noltemeyer: Organomet. 1998, 17(1), 123.

20 T. Groh: Staatsexamensarbeit. Universität Göttingen 1997.

21 U. Schneider: Staatsexamensarbeit. Universität Göttingen 1986.

22 M. Geschwentner, G. Elter, A. Meller: Z. Naturforsch. 1994, 49b, 458.

23 T. Groh: Dissertation. Universität Göttingen 2000.

24 A. Schmidpeter, K. Karaghiosoff: Heterophospholes. in M. Regitz, O. J. Scherer (Eds.): Multiple Bonds and Low Coordination in Phosphorus Chemistry. Stuttgart - New York: 1990. Georg Thieme Verlag.

25 A. Schmidpeter, K. Karaghiosoff: Azaphospholes. in H. W. Roesky (Ed.): Rings, Clusters and Polymers of Main Group and Transition Elements. Amsterdam - New York: 1989. Elsevier.

26 A. Schmidpeter: Heterophospholes. in F. Mathey (Ed.): Phosphorus-Carbon Heterocyclic Chemistry: The Rise of a New Domain. Amsterdam - New York: 2001. Pergamon.

27 K. Karaghiosoff, A. Schmidpeter: Phosphorus, Sulfur, and Silicon 1988, 36, 217.

28 N. P. Ignatova, N. N. Melnikov, N. I. Shvetsov-Shilovskij: Khim. Geterosikl. Soedin. 1967, 3(4), 753.

29 J. Luber, A. Schmidpeter: Angew. Chem. 1976, 88, 9.

$30 \quad$ R. Appel, F. Knoll, I. Ruppert: Angew. Chem. 1981, 93, 771.

31 M. Hesse, U. Klingebiel: Z. Anorg. Allg. Chem. 1983, 501, 57.

32 W. Rösch, M. Regitz: Phosphorus and Sulfur 1984, 21, 97.

33 J. H. Weinmaier, H. Tautz, A. Schmidpeter, S. Pohl: J. Organomet. Chem. 1980, 185, 53.

34 J. H. Weinmaier, G. Brunnhuber, A. Schmidpeter: Chem. Ber. 1980, 113, 335.

35 K. C. Dash, H. Schmidbaur, A. Schmidpeter: Inorg. Chim. Acta 1980, 41, 167.

36 J. G. Kraaijkamp, G. van Koten, K. Vrieze, D. M. Grove, E. A. Klop, A. L. Spek, A. Schmidpeter: J. Organomet. Chem. 1983, 256, 375.

37 J. G. Kraaijkamp, G. van Koten, D. M. Grove, G. Abbel, C. H. Stam, A. Schmidpeter: Inorg. Chim. Acta 1984, 85, L33.

38 J. G. Kraaijkamp, D. M. Grove, G. van Koten, A. Schmidpeter: Inorg. Chem. 1988, 27, 2612.

39 J. J. Daly, L. Maier: Nature 1964, 203, 1167. 
40 M. Baudler, H. Standeke, M. Borgardt, H. Strabel, J. Dobbers: Naturwissenschaften 1966, 53(4), 106.

41 M. Yoshifuji, N. Inamoto, K. Ito, S. Nagase: Chem. Lett. 1985, (4), 437.

42 J. J. Daly: J. Chem. Soc. 1964, 6147.

43 J. J. Daly: J. Chem. Soc. (A) 1966, 428.

44 R. Schmutzler, L. Heuer, D. Schomburg: Phosphorus, Sulfur and Silicon 1993, 83, 149.

45 P. S. Elmes, B. M. Gatehouse, B. O. West: J. Organomet. Chem. 1974, 82(2), 235.

46 R. Ahlrichs, D. Fenske, H. Oesen, U. Schneider: Angew. Chem. 1992, 104(3), 312.

47 H. G. von Schnering, T. Meyer, W. Hoenle, W. Schmettow, U. Hinze, W. Bauhofer, G. Kliche: Z. Anorg. Allg. Chem. 1987, 553, 261.

48 R. M. De Silva, M. J. Mays, P. R. Raithby, G. A. Solan: J. Organomet. Chem. 2002, 642(1-2), 237.

49 R. M. De Silva, M. J. Mays, G. A. Solan: J. Organomet. Chem. 2002, 664(1-2), 27.

50 S. Dielkus, C. Drost, R. Herbst-Irmer, U. Klingebiel: Angew. Chem. 1993, 105(11).

51 S. Dielkus, C. Drost, R. Herbst-Irmer, U. Klingebiel: Organomet. 1994, 13, 3985.

52 K. Bode, U. Klingebiel, H. Witte-Abel, M. Gluth, M. Noltemeyer, R. HerbstIrmer, M. Schäfer: Phosphorus, Sulfur and Silicon 1996, 108, 121.

53 U. Klingebiel, P. Werner: Liebigs Ann. Chem. 1979, 4, 457.

54 K. Knipping, C. Drost, U. Klingebiel, M. Noltemeyer: Z. anorg. Allg. Chem 1996, 622, 1215.

55 U. Klingebiel, K. Knipping, H. Witte-Abel: Z. Naturforsch. 1997, 52b, 1049.

56 M. Hesse: Dissertation. Universität Göttingen 1983.

57 N. Wiberg: Hollemann-Wiberg. Lehrbuch der Anorganischen Chemie. S. 1842. 101. Auflage. Berlin - New York: 1995. de Gruyter.

58 Autorenkollektiv: Organikum: Organisch-chemisches Grundpraktikum. 18. Auflage. S. 638. Berlin: 1990, Deutscher Verlag der Wissenschaften. 
59 K. Karaghiosoff, K. Eckstein: tert.-Butyldichlorophosphane, Dichloro(isopropyl)phosphane. in: W. Herrmann, W. Brauer (Eds.): Synthetic Methods of Organometallic and Inorganic Chemistry. Vol. 3. S. 55. Stuttgart - New York: 1996. Georg Thieme Verlag.

60 G. M. Sheldrick: Acta Crystallogr. 1990, A46, 467

61 G. M. Sheldrick: SHELXL-97. Universität Göttingen 1997 


\section{Lebenslauf}

Geboren wurde ich als einziges Kind meiner Eltern Bernt Armbruster und Christel Armbruster, geb. Pasold am 14.10.1974 in Heidelberg.

Nach dem Umzug 1978 nach Kassel besuchte ich von1981 - 1985 die Grundschule am Warteberg und von1985 - 1994 das Gymnasium Albert-Schweitzer-Schule. Im Juni 1994 erlangte ich die Allgemeine Hochschulreife.

Im Anschluss an die Schule leistete ich von 1994-1995 meinen Zivildienst an den Städtischen Kliniken Kassel im Bereich Transportpflege ab.

Zum WS 1995/1996 immatrikulierte ich mich im Fach LAG Chemie/Biologie an der Georg-August-Universität in Göttingen, absolvierte die Zwischenprüfung in Chemie nach dem WS 1997/1998 und die Zwischenprüfung im Fach Biologie im Anschluss an das SS 1998. Nachdem ich von Juni bis Dezember 2000 eine experimentelle Hausarbeit unter Leitung von Herrn Prof. Dr. A. Meller angefertigt habe, schloss ich das Studium im Mai 2001 mit dem 1. Staatsexamen für Lehramt an Gymnasien ab. Seit SS 2001 promoviere ich im Fach Chemie am Institut für Anorganische Chemie der Georg-August-Universität Göttingen und habe unter Leitung von Herrn Prof. Dr. F. Meyer und Herrn Dr. G. Elter meine Dissertation angefertigt.

Vom WS 1999/2000 - WS 2000/2001 war ich angestellt als studentische Hilfskraft für die Lehrerausbildung im Fach Chemie am Institut für Anorganische Chemie der Georg-August-Universität Göttingen. Seit SS 2001 bin ich angestellt als wissenschaftliche Hilfskraft für Forschung und Lehre. Im Sommer 2002 und Frühjahr 2003 erhielt ich die Möglichkeit bei der Phywe $\mathrm{GmbH}$ fachdidaktische Literatur zu überarbeiten und Versuche anzupassen. Anfang 2003 erhielt ich ein Stipendium der OttoVahlbruch-Stiftung.

Seit November 2003 bin ich mit Nina Helmold verheiratet. 


\section{Danksagung}

Die vorliegende Arbeit wurde in der Zeit von Juni 2001 bis März 2004 unter der Leitung von Herrn Prof. Dr. F. Meyer und Herrn Dr. G. Elter am Anorganisch-Chemischen Institut der Universität Göttingen angefertigt.

Herrn Professor Meyer möchte ich für die Unterstützung und die Bereitstellung eines Arbeitsplatzes danken.

Für seine stete Diskussionsbereitschaft, die ausgesprochen gute Betreuung, die interessante Themenstellung und unzählige NMR-Messungen danke ich Dr. G. Elter.

Für Anregungen und Diskussionen auch über Fachliches hinaus danke ich Prof. Dr. U. Klingebiel und seiner Arbeitsgruppe; insbesondere Dr. S. Kliem, T. Büschen, B. Fredelake und V. Liebau.

Dem technischen Personal des Anorganischen Institutes danke ich für die unproblematische, vielbenötigte und rasche Unterstützung.

Mein Dank gilt außerdem meiner Familie, wobei der größte Dank an meine Frau Nina geht. 